\title{
KIR gene complexity in primates
}

A genetic arsenal equipped to arm and control a killer

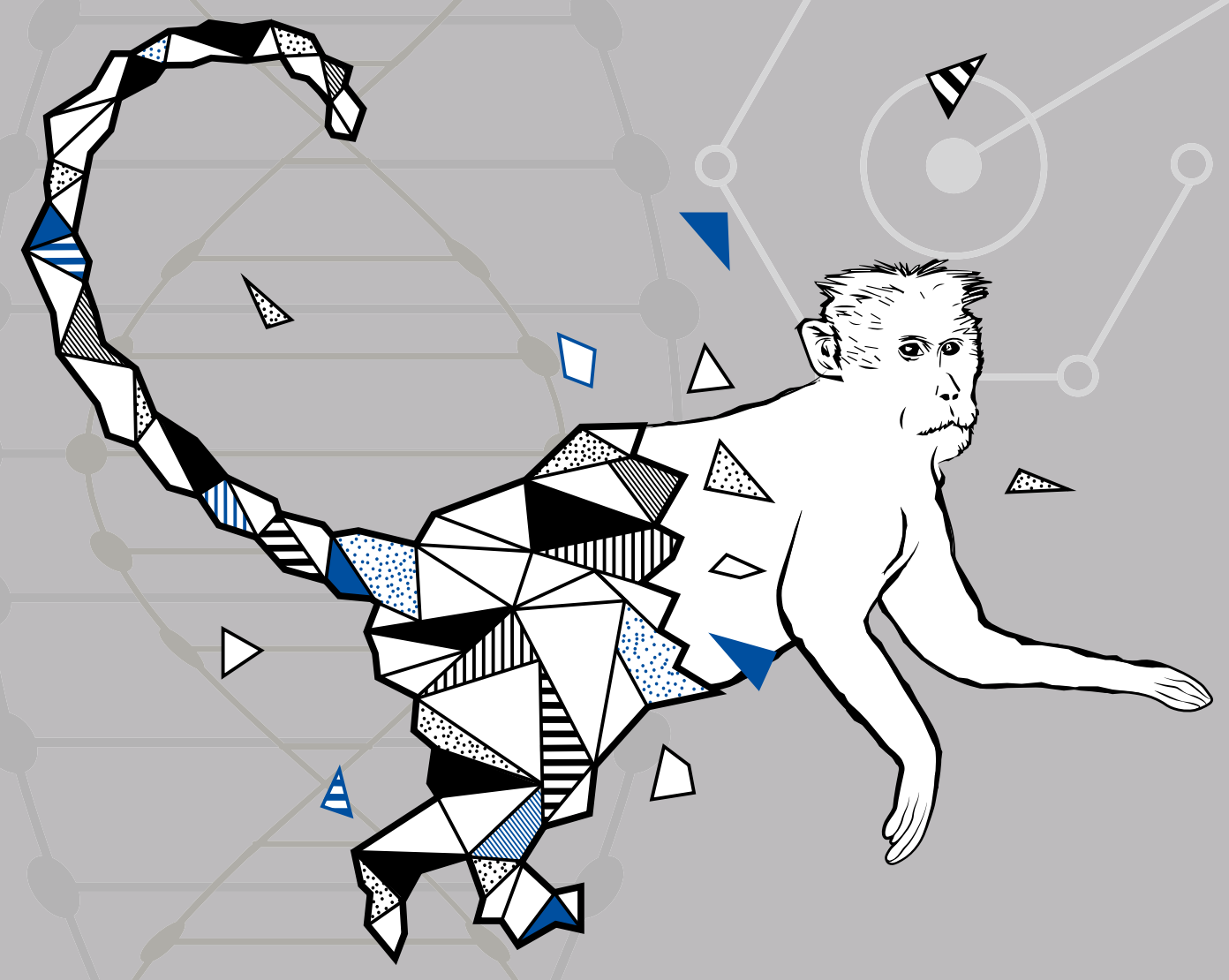

Jesse Bruijnesteijn 



\section{KIR gene complexity in primates: a genetic arsenal equipped to arm and control a killer}





\section{KIR gene complexity in primates: a genetic arsenal equipped to arm and control a killer}

\section{KIR gen complexiteit in primaten: een genetisch arsenaal om NK cellen te activeren en controleren}

(met een samenvatting in het Nederlands)

Proefschrift

ter verkrijging van de graad van doctor aan de

Universiteit Utrecht

op gezag van de

rector magnificus, prof.dr. H.R.B.M. Kummeling,

ingevolge het besluit van het college voor promoties

in het openbaar te verdedigen op

woensdag 3 november 2021 des ochtends te 10.15 uur

door

Jesse Bruijnesteijn

geboren op 28 februari 1991 te Hoorn 


\section{Promotor}

Prof.dr. R. E. Bontrop

\section{Copromotor}

Dr. N. G. de Groot

\section{Assessment Committee}

Prof. P. Parham

Prof. L. Walter

Prof. J. Kaufman

Prof. E. P. G. J. Cuppen

Prof. R. J. de Boer 
"Encumbered forever by desire and ambition, There's a hunger still unsatisfied."

David Gilmour (Pink Floyd - High hopes) 
The research described in this thesis was performed at the Dept. of Comparative Genetics and Refinement at the Biomedical Primate Research Centre, Rijswijk, the Netherlands

Financial support was provided by the Biomedical Primate Research Centre.

Illustrations: F. van Hassel

Lay-out: F. van Hassel

Cover design: Demi Bruijnesteijn

Printed by: Ridderprint BV

ISBN: 978-94-6416-837-2

(C) Jesse Bruijnesteijn, 2021. No parts of this thesis may be reproduced or transmitted, in any form, without permission in writing from the author. 


\section{Table of Contents}

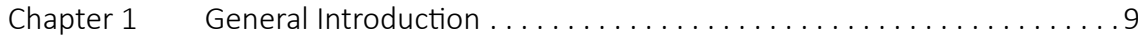

Chapter 2 Human and rhesus macaque KIR haplotypes defined by their transcriptomes ............................. 43

Chapter 3 Unparalleled Rapid Evolution of KIR Genes in Rhesus and Cynomolgus Macaque Populations. . . . . . . . . . . . . . . 69

Chapter $4 \quad$ Extensive alternative splicing of KIR transcripts $\ldots \ldots \ldots \ldots \ldots 105$

Chapter 5 Rapid characterization of complex killer cell immunoglobulin-like receptor (KIR) regions using Cas9 enrichment and Nanopore

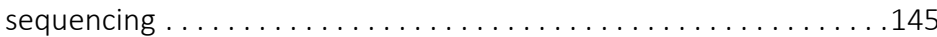

Chapter 6 The genetic mechanisms driving diversification of the KIR gene cluster in primates . . . . . . . . . . . . . . . . . . . 173

Chapter $7 \quad$ General discussion and future prospects.................205

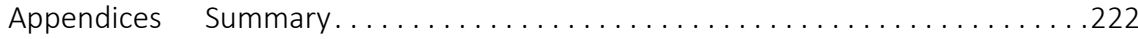

Nederlandse samenvatting . . . . . . . . . . . . . . . . . . . . . 224

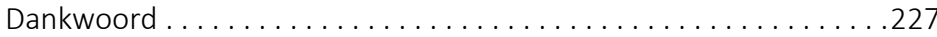

Curriculum vitae . . . . . . . . . . . . . . . . . . . . . . . 231

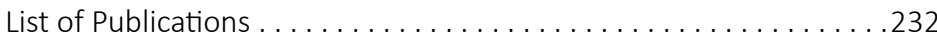





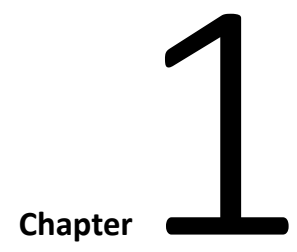

\section{General Introduction}

Jesse Bruijnesteijn 


\section{General introduction}

The killer-cell immunoglobulin-like receptors (KIR) are type I transmembrane glycoproteins that are expressed on NK cells and subsets of T cells. The KIR receptors can be categorized into activating and inhibitory forms that recognize different epitopes on MHC class I molecules. Through these interactions, the development and functional activity of NK cells are regulated. The functional capacity of NK cells is first acquired in a process termed NK cell education, which involves the recognition of self-MHC molecules by inhibitory KIR. The activity of mature NK cells is then modulated by the balanced integration of simultaneous activating and inhibitory signals. Cells with aberrant MHC class I expression, which might occur during infection or tumor formation, are recognized and subsequently lysed by the NK cells. Non-cytotoxic NK cells mainly secrete cytokines upon activation, which modulates consecutive immune responses or regulates vascular remodeling during pregnancy.

The human KIR family comprises 17 members, which vary in their structure, function, ligand specificity, expression status, and cellular localization. The genes that encode the receptors are located on chromosome 19 and are arranged in a head-totail manner. Genomic and transcriptomic characterization studies illustrated allelic polymorphism and gene copy number variation, which is further complicated by recombination events, differential haplotype configurations and alternative splicing. The characterization of the KIR gene system contributes to a better understanding of the abundant disease association studies, which demonstrated the importance of KIR in health and disease.

The characterization of KIR genes in other primate species indicates a rapid and species-specific co-evolution with their ligand-encoding MHC class / genes. The KIR receptors in different primate species share a similar structure and function, but might genetically vary in their lineage expansion and haplotype configurations. An initial characterization of the KIR gene cluster has been performed in rhesus macaques, a species that is commonly used as model in biomedical research to test new vaccines and drugs to treat human and animal diseases. In line with the extensively expanded MHC- $A$ and- $B$ genes in macaques, the macaque $K I R$ genes illustrate extensive diversity. Taking into consideration the many health and disease associations with KIR (and their MHC ligands) in humans, and the relevance of macaques as preclinical model, this manuscript is focused on understanding the KIR gene system in different macaque species.

\section{Primate taxonomy}

The definition of species, or taxonomy, is the complex study to distinguish groups of organisms, and affects the fields of genetics, population biology, ecology, and ethology [1]. At present, over 300 primate species (Primata) are recognized, which share anatomic, functional, and behavioral features that reflect common ancestry, and are 
generally divided into wet- and dry-nosed primates (Figure 1). The latter suborder includes the tarsier, monkey, and ape clades, which are further distinguished by two parvorders that separates the New World monkeys from the Old World monkeys, apes and humans. The Old World monkey superfamily (Cercopithecoidea), including the macaques, share a common ancestor with the humans and apes (Hominoidea) approximately 25-33 million years ago. The macaque genera (Macaca) comprise over 20 species, which evolutionary diverged in the past 1-3 million years (Figure 1). Even more, populations within macaque species, such as the Indian, Burmese and Chinese rhesus macaques, and the insular cynomolgus macaques, emerged by natural barriers and drove intra-species evolution.

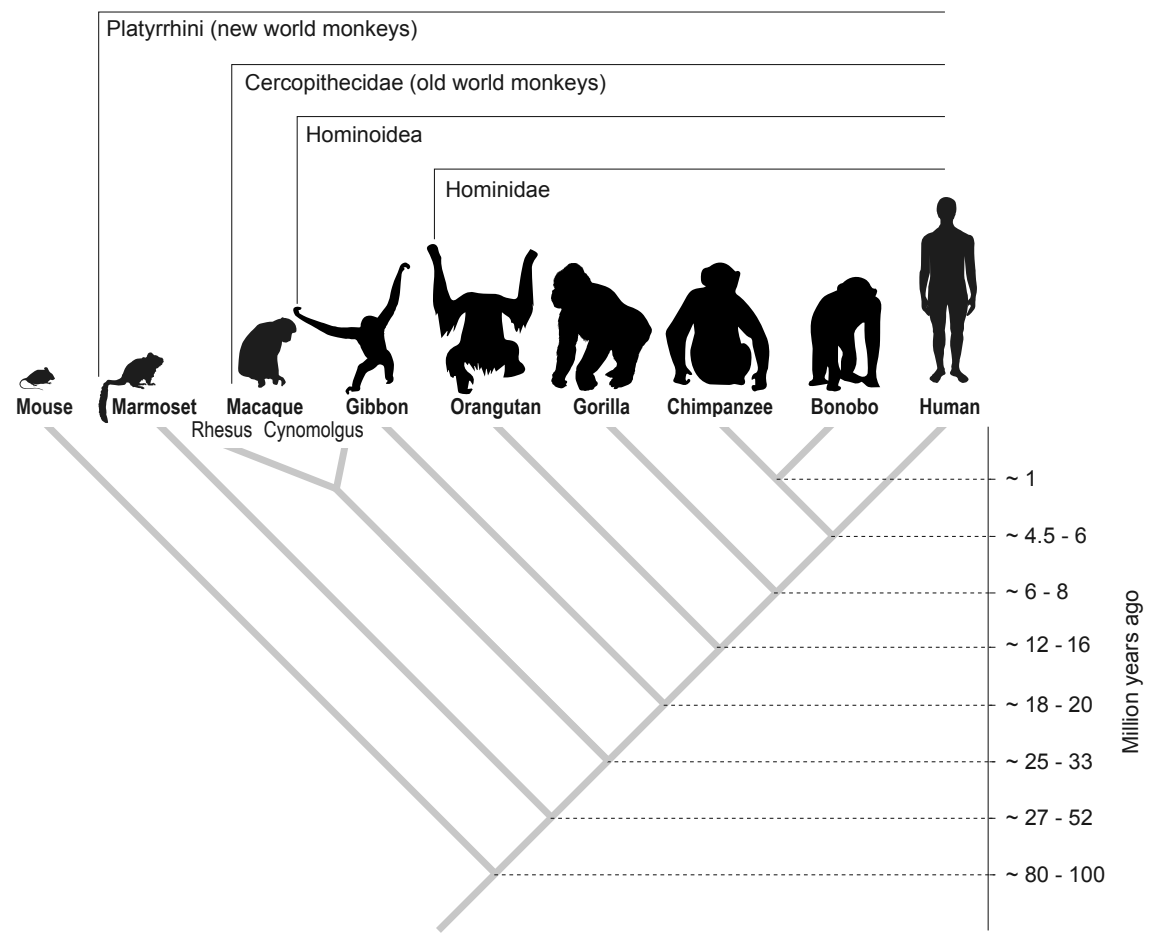

Figure 1. Evolutionary relationships and taxonomy of primates. The pedigree illustrates the estimated genetic distance to a common ancestor with humans for the different non-human primate species. Mouse has been added to display the divergence of primates from other mammals. On top, the orders and families are defined for the different primate species. 


\section{The bridge between innate and adaptive immunity}

During the evolution of species, a protective immune system was acquired that fights potentially lethal pathogens. The adaptive arm of this defense mechanism is able to specifically recognize and eliminate viruses, bacteria, fungi, and parasites, and owns a memory function to launch a more rapid response upon recurrent infection. These adaptive responses are mainly mediated by $\mathrm{B}$ - and T-lymphocytes that rely on a highly diverse repertoire of receptors, which are generated by somatic chromosomal rearrangements [2]. The B-lymphocytes mainly harbor a humoral function, by secreting antibodies and cytokines, whereas different subsets of T-lymphocytes perform regulatory and cytotoxic tasks. The evolutionary older innate arm of the immune system plays a crucial role in the initiation and activation of the adaptive immune response, and also participates in the killing and removal of pathogens by a-specific priming through interactions of generic receptors. The effectors of the innate immunity are a diverse group of lymphoid cells, which are grouped based on their cytokine production profiles and cytotoxicity [3].

Among the innate lymphoid cells are the Natural Killer (NK) cells, which were discovered in mice and were initially described for their capability to eliminate cancer cells [4-6]. This cytotoxic role is analogous to that of the $\mathrm{CD} 8^{+}$T-lymphocyte subset, although the response in NK cells is more rapid and in absence of antigen-specific priming. The lysis of target cells by activated NK cells is mediated by the engagement of death receptors, such as Fas/CD95, or by the release of small granules, which result in the secretion of perforins and granzymes. In addition to the killing of tumor and pathogen-infected cells, NK cells have immunoregulatory functions. Upon activation, they facilitate the fast production and secretion of numerous cytokines and chemokines, such as interferon (IFN) $-\gamma$, and thereby modulate both the innate and adaptive immune responses [7-9]. The NK cell response can be enhanced in the presence of different cytokines, like IL-12, IL-15, and IL-18, which are secreted by other innate immune cells. Recently, expansion of long-lasting subsets of NK cells are described in response to infectious pathogens, such as the mouse and human cytomegalovirus, which indicate memory-like characteristics for NK cells $[10,11]$. These regulatory and adaptive features, although initiated by a-specific pathogen priming, contribute to adaptive immune responses, and suggests NK cells to bridge the innate and adaptive immune system.

\section{NK cell development and subset differentiation}

In additional to shared functional features, both the NK cells and the B- and T-lymphocytes primarily originate from the common lymphoid progenitor (CLP) in the bone marrow (Figure 2). Whereas T-lymphocytes migrate and continue to develop in the thymus, the NK cells expand through the lineage-restricted NK cell progenitor (NKP), from which further differentiation is dependent on CD127 expression. Influenced 


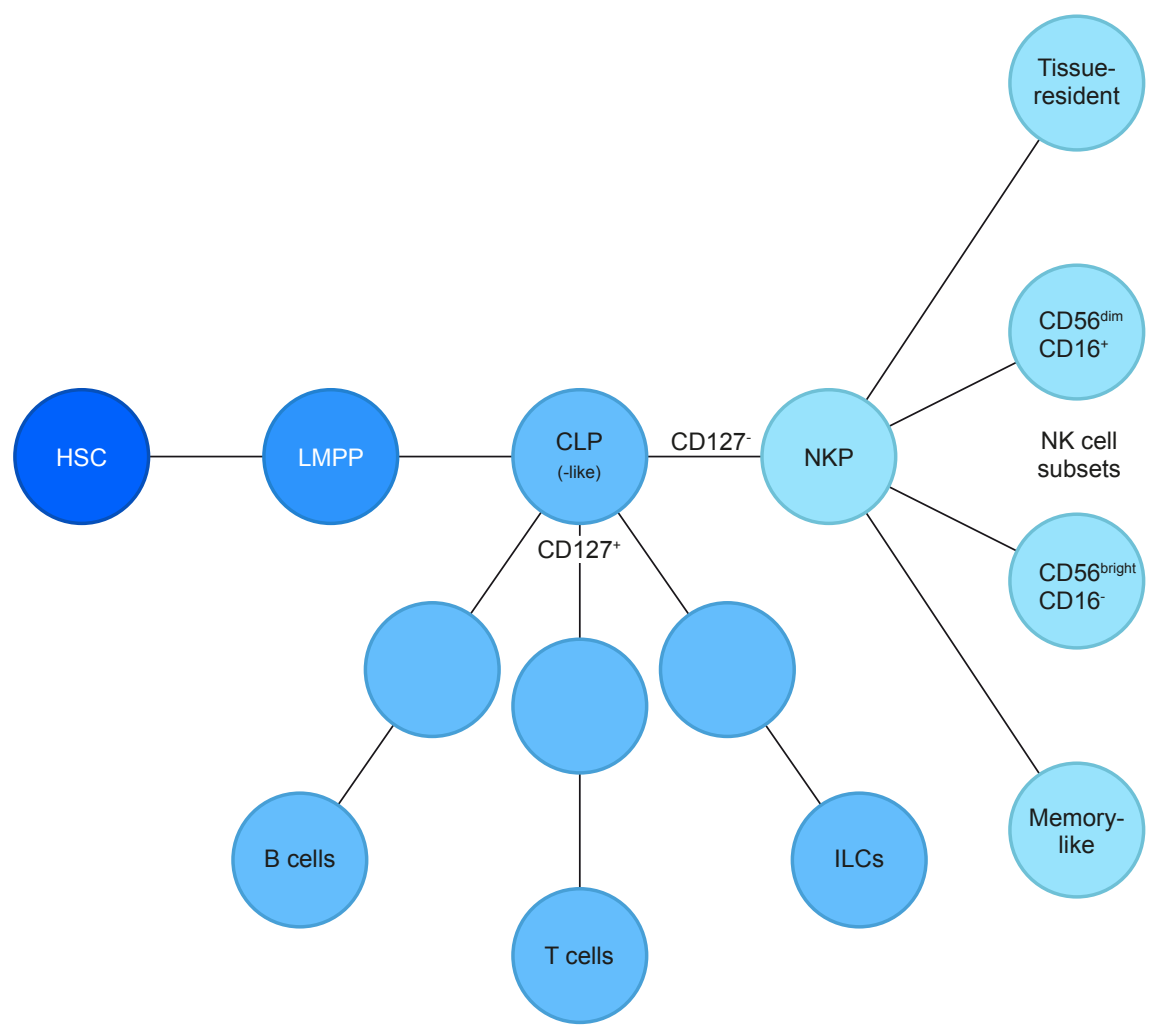

Figure 2. Development pathway of lymphocytes and NK cell subsets. Activation of hematopoietic stem cells (HSC) leads to the development of lymphoid-primed multipotent precursors (LMPP), which then differentiate into common lymphoid progenitor (CLP) cells in the bone marrow. Subsequent commitment that depends on CD127 expression generates either B- and T-cell populations and ILCs through different development stages, or a NK precursor stage (NKP). Eventually, these NKPs then differentiate into subsets of NK cells.

Table I. Four human NK cell subsets that are distinguished by specifc markers.

\begin{tabular}{|c|c|c|}
\hline Subset & Markers & Main function \\
\hline $\mathrm{CD}^{2} 6^{\operatorname{dim}} \mathrm{CD} 16^{+}$ & $\mathrm{CD}^{2} 6^{+/-}, \mathrm{CD} 16^{+}, \mathrm{KIR}^{+}, \mathrm{CD} 117^{-}, \mathrm{CD} 127^{-}$ & $\begin{array}{l}\text { Highly cytotoxic NK cells, death } \\
\text { receptors and cytolytic granules }\end{array}$ \\
\hline CD56 bright $C D 16^{-/+}$ & $\mathrm{CD}^{2} 6^{+}, \mathrm{CD} 16^{-}, \mathrm{KIR}-\mathrm{CD} 117^{+/-}, \mathrm{CD} 127^{+/-}$ & $\begin{array}{l}\text { Regulatory NK cells, secrete cytokines } \\
\text { and chemokines }\end{array}$ \\
\hline $\begin{array}{l}\text { Tissue-resident } \\
\text { CD56 bright }\end{array}$ & $\mathrm{CD}^{2} 6^{+}, \mathrm{KIR}^{\mathrm{low}}, \mathrm{CXCR6}^{+}, \mathrm{CCR}^{+}, \mathrm{CD} 49 \mathrm{a}^{+/-}, \mathrm{CD} 9^{+}$ & $\begin{array}{l}\text { Still elusive, but might shape the local } \\
\text { microenvoriment }\end{array}$ \\
\hline Adaptive NK cells & $\mathrm{CD}_{56}{ }^{+/}, \mathrm{KIR}^{+}, \mathrm{CD}^{+}, \mathrm{CD} 7^{+}, \mathrm{NKG} \mathrm{C}^{+}$ & $\begin{array}{l}\text { Memory-like expansion of cytotoxic } \\
\text { NK cells }\end{array}$ \\
\hline
\end{tabular}


by multiple transcription factors, such as GATA3, the CD127 $7^{+}$NKPs differentiate into diverse ILC populations, whereas the CD127- NKPs develop through different stages to the mature peripheral blood NK cells and NK cell subsets in tissues (Figure 2). The NK cells form a heterogenous cell population that represent $10-15 \%$ of the circulating lymphoid cells. Based on surface phenotype and cytokine profiles, NK cell subsets are defined, which - according to the prevailing linear model - represent distinct development stages. The most widely used phenotypic markers are $\mathrm{CD}^{-}{ }^{-} \mathrm{CD} 56^{+}$and $\mathrm{CD}^{-} \mathrm{CD} 16^{+}$expression, which together define two of the four major subsets in humans: $\mathrm{CD}_{5} 6^{\text {dim }} \mathrm{CD} 16^{+}$and $\mathrm{CD} 56^{\text {bright }} \mathrm{CD} 16^{-/+}$(Table I). The $\mathrm{CD} 56^{\text {dim }} \mathrm{CD} 16^{+}$subset, which represents $90 \%$ of the circulating NK cells, exerts higher natural cytotoxicity and expresses increased levels of Ig-like receptors compared to the $\mathrm{CD} 56^{\text {bright }} \mathrm{CD} 16^{-/+}$subset. Although circulating $\mathrm{CD} 56^{\text {bright }} \mathrm{CD} 16^{-/+} \mathrm{NK}$ cells can mediate cytotoxicity, the main function is the production and secretion of cytokines, such as IFN- $\gamma$, TNF- $\alpha$, and IL-10 $[12,13]$. The linear development model states that the $\mathrm{CD} 56^{\text {bright }} \mathrm{CD} 16^{-/+} \mathrm{NK}$ cells are precursors of the terminally differentiated $\mathrm{CD} 56^{\mathrm{dim}} \mathrm{CD} 16^{+}$subset [14-18]. Although this naturally cytotoxic subset predominates in blood, the majority of NK cells in the body are represented by the $\mathrm{CD} 56^{\text {bright }} \mathrm{CD} 16^{-}$cells that reside and differentiate in second lymphoid organs, such as the thymus, lymph nodes, liver, and uterus, but also in inflamed and tumor tissues [19-24]. This tissue-resident CD56 bright NK cell subset mediates specialized and tissue-specific functions, and can be distinguished from circulating NK cells by the expression of CD69, chemokine receptors (CXCR6 and CCR5), and adhesion molecules (CD49a) [25-28]. A fourth major NK cell subset can be distinguished subsequent to pathogenic encounters, such as cytomegalovirus (CMV) infection [29, 30], and comprise NK cells with memory-like or adaptive features, including robust responses upon recurrent infection and clonal expansion. These adaptive $\mathrm{CD} 56^{\mathrm{dim}} \mathrm{CD} 16^{+} \mathrm{NK}$ cells express multiple activating receptors and contain elevated levels of perforin and granzymes, which indicates high cytolytic capacity. The adaptive NK cells, which are thought to reside in the liver, poorly respond to activated immune cells and several activating cytokines, such as IL-12. Epigenetic modifications might explain the altered functional profile of this NK cell subset [31].

The broad spectrum of NK cell subsets in humans is currently thought to arise from different development stages, which hypothesize linear differentiation from a common progenitor. However, in vitro studies demonstrated NK cell differentiation from common myeloid progenitors (CMP) in the presence of NK cell-supporting cytokines, and, although artificial culture systems are used, it does speculate a branched development $[32,33]$. This is further supported by macaque studies, in which the CD56 bright and CD56 ${ }^{\text {dim }}$ NK cell subsets were acquired from different lineage progenitors $[34,35]$. Although there is still debate on the contrasting development models, the extensive subset diversity is well illustrated and hint for a broad phenotypic and functional spectrum of NK cells. 


\section{NK cell education and 'missing-self' activation}

To recognize pathogenic or infected cells and to maintain self-tolerance, NK cells are educated through a process of receptor engagements. These 'educative' interactions involve a variety of inhibitory surface receptors on the NK cells and major histocompatibility complex (MHC) class I molecules, also known in humans as human leukocyte antigens (HLA), which are ubiquitously expressed by nucleated cells and present cytosolic peptides. In a fluid and ongoing process, NK cells that express at least one inhibitory receptor that recognizes a host MHC class I molecule acquire effector functions; only these NK cells are licensed to kill [36-38]. The importance of NK cell education is demonstrated in $\mathrm{B} 2 \mathrm{~m}^{-}$mice, which lack expression of $\mathrm{MHC}$ class I molecules. The NK cells from these mice showed significantly decreased killing of MHC class I deficient target cells and abrogated cytokine production compared to NK cells from wild type mice $[39,40]$. Similarly, decreased effector functions were described for NK cells that lack MHC-specific inhibitory receptors [41]. In addition, the number of inhibitory receptors that recognize host $\mathrm{MHC}$ molecules positively correlates with the NK cell responsiveness [41, 42].

During viral infection and tumor formation, stressed cells are recognized through the presentation of pathogenic antigens by MHC class I molecules, which primes cytotoxic T-lymphocytes. This defense mechanism can be evaded by the chronic downregulation of MHC molecules from the surface of aberrant cells, which is a common evolutionary adaption for viruses and tumors [43-45]. Educated NK cells, however, detect the absence of their deterrents, and become activated to lyse the target cell. This process of NK cell activation, which has been proposed in 1981, is termed the 'missing-self' hypothesis and is thought to be the main mechanism of NK cell activation (Figure $\mathbf{3}$ ) [46].

The pool of NK cells in an individual is highly diverse, which is not only reflected by different subsets, but also on single-cell level. NK cells show a variegated expression of different activating and inhibitory receptors, which are encoded by genes that segregate independently from the $\mathrm{MHC}$-encoding genes. As a result, a significant NK cell population is unable to interact with self-MHC molecules, and, therefore, do not acquire effector functions and remain unlicensed. These uneducated cells persist in the circulation, but are hyporesponsive.

Two models were initially proposed for the differential responsiveness states of NK cells. On one hand, the 'arming' model states that the interaction of inhibitory receptors with self-MHC molecules is required for functional maturation. On the other hand, the 'disarming' model suggests that all NK cells are initially responsive, but continuous activating engagements, in the absence of inhibitory interactions with the host MHC molecules, desensitize the NK cells and result in hyporesponsiveness. A third and more supported model is the Rheostat model that describes the education procedure as a continuous process, in which the NK cell responsiveness is dependent on the quantity of environmental factors, such as the presence of absence of cytokines and MHC class I ligands. This last model would explain the persistence of unlicensed 

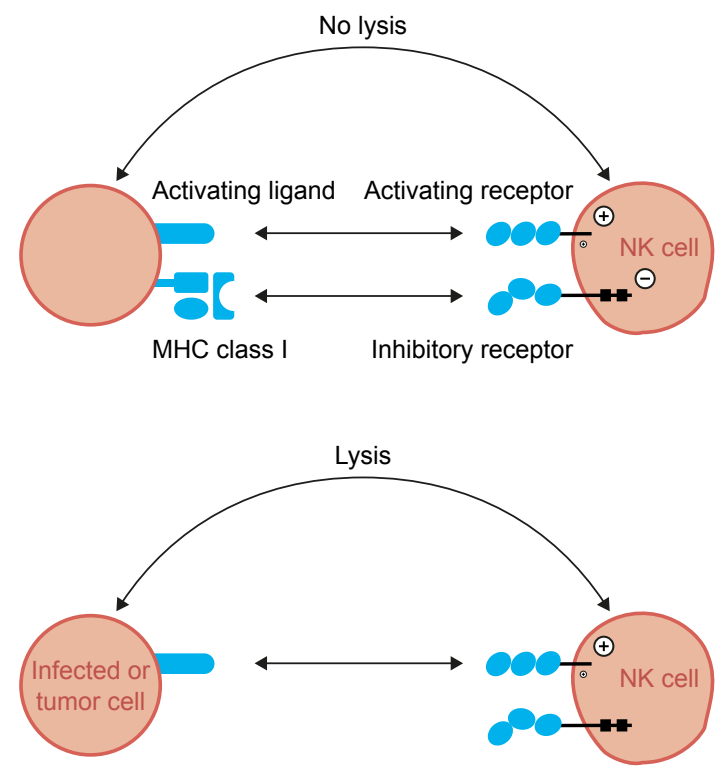

Figure 3. A schematic representation of the missing-self hypothesis. A balance of activating and inhibitory signals maintain tolerance in the upper panel through interactions of regulatory receptors expressed by NK cells and their ligands, including MHC class I. Upon infection or tumor cell formation, target cells might downregulate their MHC class I expression and the total balance of signals the NK cell receives might shift towards a more activating potential. This triggers NK cell activation and lysis of the target cell.

cells, which are thought to have effector function under specific conditions, such as viral infection and tumor formation [47, 48], and can be 're-educated' upon environmental changes [37, 49].

\section{NK cell receptors}

A broad spectrum of activating and inhibitory receptors is expressed on the surface of the NK cell and cooperate with each other to regulate the cytotoxicity. The transmembrane and cytoplasmic domains determine the signaling potential of these receptors. In general, inhibitory receptors contain one or multiple immunoreceptor tyrosine-based inhibitory motifs (ITIM) in their cytoplasmic tail, whereas activating receptors possess a positively charged residue in their transmembrane region that can interact adaptor molecules, such as DAP10, DAP12, and FcR- $\psi$. These adaptors contain immunoreceptor tyrosine-based activation motifs (ITAM), which transduce activating signals. 
The majority of activating and inhibitory receptors belong to three receptor families that contribute to NK cell regulation. The Natural Cytotoxicity Receptor (NCR) family comprise only activating receptors, namely NKp46, NKp44, and NKp30, and are known to interact with several non-MHC molecules, such as heparan sulfate glycosaminoglycans [50]. NKp46 and NKp30 are constitutively expressed on NK cells, but are also identified on subsets of ILCs and T cells. Another family involved in NK cell regulation is the calcium-dependent lectin-like receptor family that includes CD94-NKG2-A/C/E/F/H heterodimers and NKG2D homodimers, which are inhibitory and activating receptors, respectively. These inhibitory receptors interact with several non-classical MHC molecules, whereas multiple other ligands are documented for NKG2D, including the major histocompatibility complex class I chain-related molecules (MIC-A/B) [51, 52]. The third receptor family comprise the killer cell immunoglobulin-like receptors (KIR), which form a highly diverse and expanded group of inhibitory and activating receptors expressed on NK cells and subsets of T cells.

\section{KIR discovery, structure and nomenclature}

After the 'missing-self' hypothesis was proposed in 1981, researchers started to work on the question how cells with an aberrant $\mathrm{MHC}$ expression were recognized and killed by the NK cells. Transfection studies demonstrated that the loss of HLA-A,-B, and $-C$ expression resulted in susceptibility to NK cell-mediated lysis [53, 54]. This susceptibility was reversed by the transfection of certain HLA class I genes, indicating a molecular interaction of HLA molecules with proteins present on NK cells. Subsequently, multiple immunoglobulin-superfamily receptors were cloned to demonstrate putative interactions, including the receptors now known as KIR [55-57]. However, before standard nomenclature was introduced in 1996 [58], many different names were given to these receptors, including p50, p58 and p70, which referred to their molecular weight determined by immunoprecipitation, and the NK-associated transcripts (NKat). Even more, the acronym KIR first referred to killer inhibitor receptors, and was later changed to killer cell immunoglobulin-like receptors when the activating family members were discovered [59, 60].

The KIR family comprises receptors that consist of up to three extracellular domains, followed by a stem region, a transmembrane region, and a cytoplasmic tail (Figure 4). The nomenclature of the KIR receptors helps to distinguish differential structures. The first digit following the KIR abbreviation indicates the number of extracellular domains (denoted as ' $D$ '). In humans, KIR2D and KIR3D structures are reported, whereas in several Old World monkeys KIR1D structures are documented as well. The length of the cytoplasmic can either be long or short, denoted with an ' $L$ ' or ' $S$ ', and characterizes inhibitory or activating KIR, respectively, whereas a ' $P$ ' denotes a pseudogene. A sequential two-digit numbering distinguish the different $K I R$ genes. Non-synonymous $K I R$ alleles are distinguished by three-digit numbers that are separated from the gene digits by an asterisk, whereas synonymous polymorphisms in the coding sequence of a 


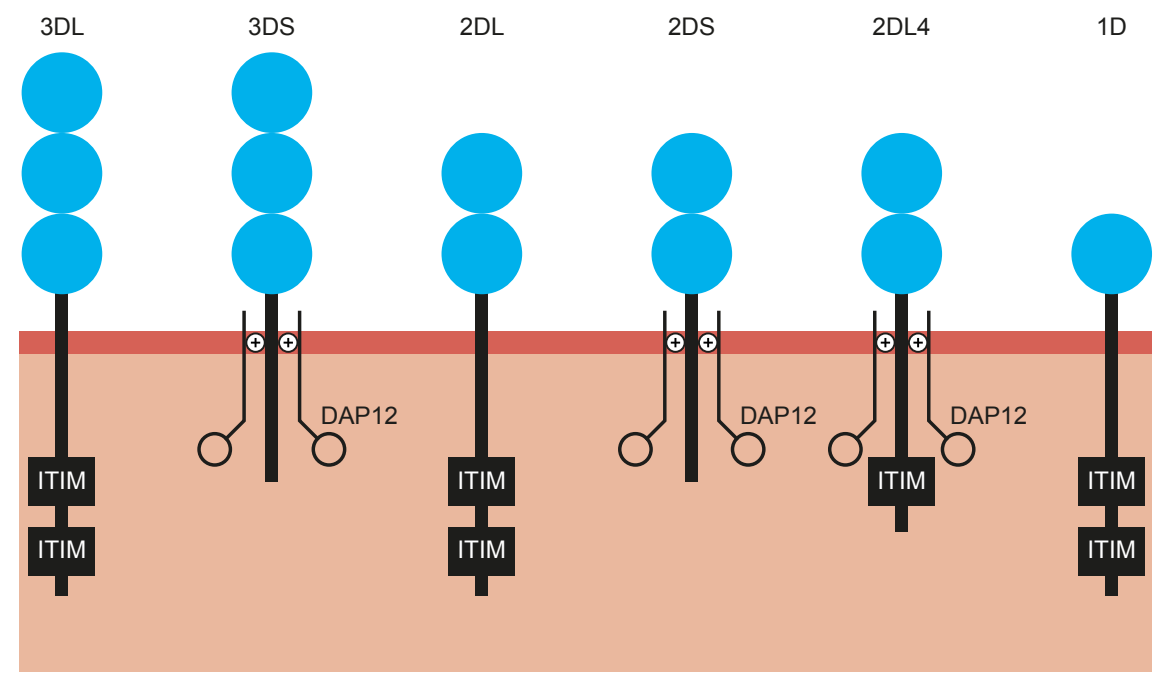

Figure 4. A schematic overview of the KIR receptor structure and nomenclature. KIR receptors display differential structures, with 2 or 3 extracellular domains, to which is referred to with KIR2D or KIR3D in nomenclature, respectively. In some primate species, receptors with a single extracellular domain are documented (KIR1D). A long (L) cytoplasmic tail can contain two immunoreceptor tyrosine-based inhibitory motifs (ITIM) and represent inhibitory receptors (e.g. KIR3DL). Short (S) cytoplasmic tails have a positive residue present in their transmembrane region (e.g. KIR3DS), which can interact with adaptor molecules, such as DAP12, that contain immunoreceptor tyrosine-based activation motifs (ITAM). An exception is KIR2DL4, which has both activating and inhibitory signaling potential.

KIR gene are distinguished by a second set of two digits (e.g., KIR3DL1*001:02). Optional suffixes can be provided to denote the expression and recombination status of a receptor $[61,62]$. These nomenclature guidelines apply to the KIR gene system in all primates, with a few species-specific exceptions.

\section{The genetic cluster}

The genes encoding the human KIR receptors are located on chromosome 19q13.4 within the leukocyte receptor complex (LRC) and span a $100-200 \mathrm{~kb}$ region. The length of a single $K I R$ gene is approximately $10-14 \mathrm{~kb}$ and the different genes are separated by intergenic regions of about 2-3 kb. The receptor is encoded by up to 9 exons, of which the first 2 encode the leader peptide, and exons 3-5 encode the extracellular domains (D0, D1 and D2). The stem region is encoded by exon 6, whereas exons 7-9 encode the transmembrane region and the cytoplasmic tail.

In addition to the KIR cluster, the LRC harbors more genes belonging to the immunoglobulin-like superfamily. For example, at the centromeric side the KIR gene 
cluster is flanked by genes that encode the leukocyte immunoglobulin-like receptor (LILR) family, which are predominantly expressed in different lymphoid and myeloid cell types. The LILR family seems to be involved in the regulation of a diverse set of immune cells through interactions with $\mathrm{MHC}$ and non-MHC ligands [63]. Other structurally related members located in the LRC are the leukocyte-associated immunoglobulin-like receptors (LAIR), the sialic acid-binding immunoglobulin-type lectins (SIGLEC), the NCR1 (also referred to as NKp46), and the Fc- $\alpha$ receptor (FCAR), the latter of which is flanking the KIR genes at the telomeric side [64, 65]. In the extended LRC region, genes are identified that encode the ITAM-containing adapter molecules, which interact with activating immunoglobulin-like receptors, such as DAP10 and DAP12.

The KIR gene region is subjected to expansion and contraction, which results in a variable number of genes present within the cluster. The $K I R$ genes display a high degree of similarity and are tandemly arranged in a head-to-tail manner. In primates, however, one conserved KIR gene is located outside the KIR cluster, namely KIR3DX1, which is identified within the LILR region. Phylogenetic analysis illustrates that KIR3DX1 belongs to a different ancient lineage then the other KIR genes, and share a common progenitor $135.5 \pm 10.5$ million years ago [66]. Since then, the KIR3DL/S genes expanded and diverged by recombination, deletion and inclusion events, whereas the KIR3DX1 remained a conserved pseudogene. In contrast, the KIR3DX lineage is expanded in cattle $[66,67]$. The expansion of the KIR3DL/S genes resulted in four defined phylogenetic lineages that distinguish the receptors in their structure and MHC class I specificity. This lineage diversification probably predates the primate speciation, indicating that the KIR genes in different primate species represent the same lineages. However, after speciation, the KIR genes have diverged, which resulted in separately clustered and species-specific genes.

\section{The human KIR repertoire and haplotypes}

In humans, 17 KIR genes are documented, of which two are identified as pseudogenes, namely KIR2DP1 and KIR3DP1. The expressed KIR genes are divided into four different lineages; lineage I includes KIR2DL4 and KIR2DL5(A/B), linage II includes KIR3DL1, KIR3DL2 and KIR3DS1, lineage III includes KIR2DL1, KIR2DL2, KIR2DL3, KIR2DS1, KIR2DS2, KIR2DS3, KIR2DS4, KIR2DS5, and the pseudogenes, and lineage $V$ includes KIR3DL3.

Both lineages I and III include receptors with two extracellular domains, but these are differently encoded. The main difference is within the extracellular domains: KIR2DL4 and KIR2DL5 (lineage I) contain the D0 and D2 domains, whereas the lineage III KIR genes express the D0 and D1 domains [68]. The KIR2DL4 further differs from the remaining receptors, as the long cytoplasmic tail only contains a single ITIM, in contrast to two ITIMs in the tail of all other inhibitory KIR. In addition, KIR2DL4 possess a positively charged residue in the transmembrane region, like in all activating KIR, which 
can interact with ITAM-containing adaptor molecules. This provides KIR2DL4 with both inhibitory and activating signaling potentials [68-70].

Another atypical receptor is KIR3DL3, which is likely to encode an inhibitory receptor with 3 Ig-like domains, but lack the stem region (exon 6) and contain only a single ITIM in the cytoplasmic tail [71]. This protein is only expressed at low levels, but can be upregulated by treatment with methyltransferase inhibitors, which indicates that the promotor activity is regulated by methylation $[72,73]$. A recent study also demonstrated regulation of KIR3DL3 expression levels via different miRNAs [74]. The receptor seems to have emerged from an ancient recombination event, as the domain-encoding exons cluster into lineage $\mathrm{V}$, whereas the cytoplasmic tail is more similar to that observed in lineage III KIR. This structure is conserved in primates, including the Old World monkeys. In addition, KIR3DL3 is identified in all individuals from different geographically and ancestrally human populations that were studied for their KIR repertoire [75], which indicates an essential function for this receptor. The function and ligands of KIR3DL3 are, however, not discovered yet.

The human KIR haplotypes can be categorized into two groups, termed $A$ and $B$ haplotypes. Four framework genes form the basis of most haplotype configurations and include KIR3DL3, KIR3DL2, KIR3DP1, and KIR2DL4. A recombination hotspot in the center of the haplotype distinguishes a centromeric and telomeric region. In humans, the KIR genes expanded in both of these haplotype regions. The two haplotype groups have different KIR gene content: group A haplotypes contain a fixed set of 7 KIR genes, of which only a single gene encodes an activating receptor (KIR2DS4), whereas group $B$ haplotypes contain a variable number of genes, including multiple activating receptors. Haplotype configurations that contain both group $A$ and $B$ haplotype content can result from chromosomal recombination events. The two standard $A$ and $B$ configurations are documented in all populations, but with different relative frequencies that correlate with the frequencies of the KIR ligands [76].

\section{KIR ligands}

As mentioned, interactions of NK cell receptors with proteins on target cells are involved in NK cell education, pathogen recognition and subsequent immune response initiation. The KIR receptors play a central role in these processes, as the individual KIR receptors recognize distinct $\mathrm{MHC} / \mathrm{HLA}$ allotypes. In humans, the HLA genes are located on chromosome 6 and are classified as class I, II, and III. The HLA class II genes encode molecules that are present on specialized immune cells, such as dendritic cells and present extracellular proteins. The structure and function of HLA class III molecules are less defined, but involve cell signaling. The HLA class I molecules, which include ligands for KIR, are encoded by six genes: $H L A-A,-B,-C,-E,-F$, and $-G$. The first three genes (classical) are expressed on almost all nucleated cells and are characterized by extensive polymorphism, whereas the latter three genes (non-classical) show more restricted expression and encode conserved receptors. Some of the non-classical HLA molecules 
are described as ligand for KIR. For example, KIR2DL4 expressed in endosomes of uterine NK cells might interact with soluble HLA-G [77], whereas open conformers of HLA-F are suggested to interact with KIR3DS1 [78].

However, most ligands of KIR belong to the classical HLA class I molecules (Table II) [57, 77-92]. The KIR receptors interact with specific epitopes that are present on HLA-A, $-B$ and $-C$ molecules, which are termed A3/A11, Bw4, C1, and C2. The A3 and A11 epitopes are identified on approximately $45 \%$ of the HLA-A molecules, whereas about $36 \%$ of the HLA-B molecules, and a few HLA-A alleles, contain a Bw4 epitope [93]. The majority of the HLA-A and-B molecules do not function as ligand for KIR, but rather evolve under the pressure of the T-cell response. In contrast, all HLA-C molecules facilitate interactions with human KIR as they possess either an C1 or an C2 epitope. $H L A-C$ is the most recently evolved HLA gene and probably resulted from a duplication of an HLA-B-like entity that encoded an C1 epitope [94]. A general overview of the HLA and KIR interactions are provided in table II.

Table II. Ligand specificity of human KIR for different HLA molecules. The open conformer status of HLA-F is abbriviated as OC.

\begin{tabular}{|c|c|c|c|c|c|}
\hline Lineage & KIR & Signal & HLA ligand & Epitope & Reference \\
\hline \multirow{3}{*}{ Lineage I } & KIR2DL4 & Inhibitory/Activating & HLA-G & - & [77] \\
\hline & KIR2DL5A & Inhibitory & Unknown & - & [88] \\
\hline & KIR2DL5B & Inhibitory & Unknown & - & [88] \\
\hline \multirow{10}{*}{ Lineage III } & KIR2DL1 & Inhibitory & HLA-C (some HLA-B) & $\mathrm{C} 2$ & {$[85-87,92]$} \\
\hline & KIR2DL2 & Inhibitory & HLA-C (some HLA-B) & $\mathrm{C} 1$ & {$[83-85,87]$} \\
\hline & KIR2DL3 & Inhibitory & HLA-C (some HLA-B) & $\mathrm{C} 1$ & {$[83-85,87]$} \\
\hline & KIR2DS1 & Activating & $\mathrm{HLA}-\mathrm{C}$ & $\mathrm{C} 2$ & {$[85,92]$} \\
\hline & KIR2DS2 & Activating & HLA-A and $-\mathrm{C}$ & Peptide-dependent & {$[82]$} \\
\hline & KIR2DS3 & Activating & Unknown & - & [84] \\
\hline & KIR2DS4 & Activating & HLA-A (some HLA-C) & A11 & {$[79,81]$} \\
\hline & KIR2DS5 & Activating & Unknown & - & {$[80,84]$} \\
\hline & KIR2DP1 & Pseudogene & - & - & [89] \\
\hline & KIR3DP1 & Pseudogene & - & - & {$[90]$} \\
\hline \multirow{3}{*}{ Lineage II } & KIR3DL1 & Inhibitory & HLA-A and-B & Bw4 & {$[57]$} \\
\hline & KIR3DL2 & Inhibitory & HLA-A & $\begin{array}{l}\text { A3/A11 } \\
\text { peptide-dependent }\end{array}$ & {$[91]$} \\
\hline & KIR3DS1 & Inhibitory & HLA-B and-F & HLA-F: OC & [78] \\
\hline Lineage $\mathrm{V}$ & KIR3DL3 & Inhibitory & Unknown & - & [73] \\
\hline
\end{tabular}


The specificity and avidity of the KIR-HLA interactions can, however, vary for different alleles, and might also be dependent on the peptide bound by the HLA molecule. For example, KIR2DL1 molecules are generally able to interact with HLA-C2 epitopes, but the KIR2DL1*022 allele switched its specificity to the C1 epitope [95]. In addition, different KIR2DL1 alleles are reported to interact with variable avidity to C2 epitopes. The importance of HLA-bound peptides is demonstrated by differential specificity and avidity of the interactions with KIR, and provide a mechanism to recognize changes in the peptide content, which may occur during viral infection or tumor formation $[96,97]$.

For several KIR receptors the ligand is not identified. They might utilize specialized interactions under specific conditions, like infection and tumor formation, or during development. Also, non-MHC molecules might interact with $\mathrm{KIR}$, like is demonstrated for heparan sulfate and KIR2DL4 [98]. This interaction directly modules the cellular localization of KIR2DL4 and might also affect its function.

\section{KIR in disease and pregnancy}

Given the independent segregation of the highly polymorphic KIR and HLA gene families, and their important role in modulating the innate and adaptive immune responses, the diversity of KIR-HLA interactions might influence the health and disease of an individual. However, the extensive diversity of KIR-HLA interactions and the incomplete knowledge of the KIR ligands make the interpretation of disease association studies difficult. Nevertheless, abundant genomic studies have provided insights in the role of KIR in disease.

Associations with a variety of infectious diseases are reported [99-101], of which most infection studies involved the effect of KIR genetics on the progression of HIV. These studies mainly determined the control of HIV infection in the presence or absence of specific KIR genes, with special interest for KIR3DL1 and KIR3DS1. For example, interactions of certain KIR3DLI and HLA-B allotypes seem to protect from HIV progression [102], and the presence of both KIR3DL1 and KIR3DS1 further suppress the viral load [103]. Even more, the presence of KIR2DL2, KIR2DL5, KIR2DS5, and KIR2DS2 in the maternal KIR repertoire is associated with a decreased HIV transmission to the child [103]. An meta-analysis of 13 cohort studies, however, demonstrated that only the presence of KIR2DL3 and KIR3DS1 (homozygous) in the Caucasian subpopulation was associated with protection against HIV progression [104].

The role of KIR in different forms of cancer is extensively studied [105-109]. For example, an increased frequency of KIR2DL2 and KIR2DS2, both linked to KIR haplotype $B$, was reported in patients with leukemia. Therefore, it was suggested that the inhibitory KIR profile of haplotype A was protective against leukemia [110]. In a different cohort of leukemia patients it was shown that the presence of KIR2DL2 was not significantly higher in patients, but instead KIR2DS2 and KIR2DS4 showed a positive correlation with disease [111]. In opposition, a model has been proposed that suggests 
a protective role of $K I R 2 D L 2$ in leukemia patients [112]. The contradicting results illustrate the difficulty to associate KIR with different forms of cancer [113]. These association studies are mainly based on the presence or absence of KIR genes and the corresponding disease progression, but might miss information on the tumor phenotype, HLA ligands, KIR haplotype configurations, and KIR alleles, which might influence the disease status. However, a more tailored approach might provide opportunities to target the KIR-HLA interactions in certain types of cancer [105].

Other disease association studies involved inflammatory and autoimmune disorders [114-117], or the effect of KIR in organ and stem cell transplantation [118-122].

During pregnancy, the local immune system is involved in vascular remodeling and in avoiding the rejection of the foetus, which possesses paternal antigens. Specialized NK cells that reside in the uterine (uNK cells) might play an important role by secreting cytokines and chemokines upon binding of KIR with HLA-C molecules, which are present on trophoblasts. Association studies illustrated an increased risk of pregnancy disorders for certain maternal inhibitory KIR and fetal HLA-C combinations [123-126]. Also, the absence of activating KIR, like KIR2DS1 and KIR2DS4, might result in poor trophoblast invasion, placental stress, and growth restriction due to insufficient NK cell activation and subsequent cytokine release [127, 128]. In contrast, however, the presence of activating KIR, including KIR2DS1, was also associated with increased risk of recurrent miscarriage $[129,130]$. In addition to the lineage III KIR receptors, KIR2DL4 might also play a role in early pregnancy through interactions with soluble HLA-G, which is predominantly expressed in fetal trophoblast cells [77, 131].

The majority of the association studies are based on the presence or absence of KIR and HLA genes. The outcome of the studies might be influenced by additional factors, such as allelic variation, disease phenotype, missed recombinant genes, potential other ligands, and genetic variation of populations and individuals.

\section{NK cells in animal models}

The involvement in essential immunological processes, such as the killing of aberrant cells, but also the associations with pregnancy, therapies and diseases, underlines the need for a better understanding of NK cells and their receptors. Studies in humans are mainly performed on peripheral blood lymphocytes, which represents a single subset of NK cells. More specialized NK cells that reside in tissues are less accessible. In addition, controlled disease experiments in humans are challenging and unethical, and are therefore limited to patient cohort studies. The contradicting results in the association studies and the restrictions in human studies illustrate the urgency for models to study NK cells in health and disease.

Studies in murine models elucidated several basic principles of the NK cells, such as the 'missing self' hypothesis and memory-like functions [46, 132]. These findings demonstrate that mouse models can be a powerful model to study fundamental processes. However, hardly any outcomes of mouse studies that involved NK cells were 
of medical relevance, which might be explained by the substantial differences in human and mouse NK cell biology. Significant variance is observed for the cytotoxicity, subsets, localization, and cytokine production profiles of the NK cells in both species [133]. The major difference is, however, within the receptors that are expressed. The MHC class I molecules are recognized by Ly49 molecules in mice, whereas in humans KIR facilitate these interactions $[134,135]$. Although Ly49 and KIR have similar functions (analogs), their structure, genetics, and $\mathrm{MHC}$ binding sites are different and are not comparable. In addition, the structure and function of the inhibitory NKG2A and activating NKG2D receptors are highly conserved, but the ligands diverged in humans and mice, indicating a diverged immune response [136]. Humanized mouse models might improve immunological studies, although the applicability may be limited for lymphocyte studies $[137,138]$. In overview, mouse models provide insights into the basic principles of NK cell immunology, but, considering the broad differences, might be less useful in disease-related studies.

Translational biomedical research with non-human primate species has played an important role in the advances of the medical, pharmaceutical, and scientific field. Macaque species share a relatively close common ancestor with humans that lived approximately 25 million years ago, which explains the similarity to humans in their physiology, reproduction, development, and immunology. Rhesus and cynomolgus macaques, two species that diverged from each other 1-3 million years ago, are widely used as model in infectious and autoimmune diseases, including models for HIV/SIV, tuberculosis, and zika infection [139-141]. Although differential properties are reported for several immune cells in primate species [142], the macaque and human NK cells share abundant characteristics [143]. Three NK cells subsets are defined in macaques, of which the $\mathrm{CD} 56+\mathrm{CD} 16$ and $\mathrm{CD} 16^{+}$populations resemble the CD56 bright and $\mathrm{CD} 56^{\mathrm{dim}}$ human NK cell subsets, respectively [144]. The third macaque subset is double negative (CD56 $\mathrm{CD}^{-} \mathrm{6}^{-}$) and might be an intermediate NK cell population. In addition, like in humans, tissue-resident NK cells are described in macaques, although data is limited $[145,146]$. The activity of macaque NK cells is regulated by inhibitory and activating receptors, including a highly diverse set of KIR receptors that recognizes $\mathrm{MHC}$ class I molecules. These characteristics indicate that macaque species are suitable biomedical models in studies that involve NK cells.

\section{Co-evolution of the MHC and KIR genes in hominids}

The KIR gene region show considerable variation and expansion across different primate species, as do the genes that encode their MHC class I ligands. Of these ligands, $\mathrm{MHC}-\mathrm{C}$ is the most recently evolved member and share orthologs in humans, chimpanzees, bonobos, and orangutans [147]. In humans, the HLA-C molecules are fixed and their $\mathrm{C} 1$ and $\mathrm{C} 2$ epitopes act as dominant ligands for the lineage III KIR, whereas the more ancient HLA-A and-B are only recognized by a few KIR [148]. In contrast, in orangutans, MHC-C is present on half of the MHC haplotypes and only have 
the C1 epitope [149]. The orangutan KIR show specificity for the C1 epitopes, but not $\mathrm{C} 2$ epitopes. This indicates that $\mathrm{MHC}-\mathrm{C}$ emerged in a common ancestor of humans and orangutans, and that HLA-C and KIR further evolved in humans to gain C2 epitopes and specific KIR ligands. This rapid and species-specific co-evolution is substantiated in chimpanzees by the presence of KIR receptors specific for both $\mathrm{C} 1$ and $\mathrm{C} 2$ epitopes [150].

The rapid evolution of the KIR genes is further illustrated by the presence of only a few orthologs in hominids. Chimpanzees and humans diverged from a common ancestor relatively recently, but share only four orthologs, namely KIR2DL4, KIR2DL5, KIR2DS4, and KIR3DL3 [93]. In orangutans, similar orthologs are identified, with the exception of KIR2DS4. The majority of the KIR genes are, however, species-specific. In humans, both the telomeric and centromeric haplotype regions show an expansion of genes [151], whereas the chimpanzee and orangutan KIR genes are mainly confined to the centromeric region [93]. Even more, the standard A and B configurations observed in humans is absent in other primates, which might indicate an evolutionary selective factor that drive the differentially distributed haplotype groups [76].

A comparable framework is described for the KIR haplotypes in hominids, which have similar genes flanking the centromeric and telomeric regions. This suggests an ancestral haplotype that was generated by duplications before speciation [149, 152]. On one side, the centromeric region is flanked by KIR3DL 3 in all hominids, which was probably generated by a recombination event [153], whereas the other side is marked by a pseudogene. The sequence of this pseudogene encodes the first 5 exons in all great apes and is followed by repetitive elements. The recombination events that resulted in the pseudogene are, however, species-specific, with genes from distinct lineages involved. This framework pseudogene is therefore not considered as an ortholog in primate species. The telomeric region is flanked by KIR2DL4 and KIR3DL2/ $K I R 3 D L 1$. The former gene is largely conserved in primates, with little species-specific variation. The number of ITIM motifs in the cytoplasmic tail of KIR2DL4 might, however, vary in different species, with either one or two motifs present [154]. The boundary of the KIR gene cluster, which is adjacent to the FCAR gene, is marked by a lineage II KIR gene in all great apes, although this gene is not conserved.

\section{$K I R$ genes in macaques}

Comparative analysis of the KIR gene cluster in different higher primate species highlighted shared characteristics, although species-specific evolution was evident. At least in part, the diversification of the KIR gene system can be explained by co-evolution with the MHC class I ligands. In contrast to higher primates, however, macaques do not have a homolog of $\mathrm{MHC}-\mathrm{C}$, and instead have an expanded repertoire of $M H C-A$ and- $B$ genes [155-157]. In agreement with the absence of $M H C-C$, just a single lineage III KIR gene is described in macaques, which is transcribed as a receptor with a single extracellular domain (KIR1D) [158, 159]. Macaque MHC haplotypes can contain up to 
3 MHC-A genes and even more copies of $M H C-B$, both of which are characterized by abundant polymorphism. Several rhesus macaque lineage II KIR are identified to interact with MHC-A, and a few MHC-B (Table III), which is in line with an extensive expansion reported for this KIR lineage in macaques [160-163]. In total, 22 rhesus macaque KIR genes were described before this manuscript was published, of which 18 clustered into lineage II $[61,164,165]$. These lineage II KIR genes encode receptors with three extra cellular domains (KIR3DL and KIR3DS). Genomic and transcriptomic segregation studies defined several rhesus macaque KIR haplotypes that contained 6 to 13 KIR genes, indicating copy number variation [166]. KIR3DL20 was reported on the majority of the defined haplotypes, suggesting a framework gene status. This gene probably shares a common progenitor with human $K I R 3 D L 3$, as the exons that encode the extracellular domains are similar [153]. The stem region and cytoplasmic tail are, however, encoded by exons that cluster into a different lineage. The pseudogene that

Table III. Rhesus macaque KIR interactions with MHC molecules. This overview represents a generalization, as the specificity for epitopes seems to be allele specific, which are described in more detailed in the corresponding literature.

\begin{tabular}{llllll}
\hline Lineage & KIR & Signal & MHC ligand & Epitope & Reference \\
\hline & KIR3DL01 & Inhibitory & Mamu-B & Bw4 & {$[161]$} \\
\cline { 2 - 6 } & KIR3DLW03 & Inhibitory & Mamu-A1 & Bw4 & {$[160]$} \\
\cline { 2 - 6 } & KIR3DL05 & Inhibitory & Mamu-A1,-A3,-B,-AG & Bw4, Bw6 & {$[160,162,163]$} \\
\cline { 2 - 6 } Lineage II & KIR3DL06 & Inhibitory & Mamu-B & Bw4 & {$[163]$} \\
\cline { 2 - 6 } & KIR3DL08 & Inhibitory & Mamu-B & Bw4 & {$[163]$} \\
\cline { 2 - 6 } & KIR3DL11 & Inhibitory & Mamu-A1 & - & {$[160]$} \\
\cline { 2 - 6 } & KIR3DS05 & Activating & Mamu-A1 (very weak) & - & {$[160]$} \\
\cline { 2 - 6 } & KIR3DSW08 & Activating & Mamu-A3 (very weak),-B & Bw4 & {$[160,163]$} \\
\hline
\end{tabular}

might be present on the ancestral KIR haplotype is also reported in rhesus macaques, although its framework status is not confirmed. The documented rhesus macaque pseudogene (KIRDP) is not orthologous to human KIR3DP1, as the first three exons associate with different rhesus macaque sequences [149]. On approximately half of the haplotypes, KIR2DLO4 is identified, which represents the only macaque KIR ortholog that is shared with humans and other hominids [166]. All macaques KIR2DL04 allotypes contain two ITIMs in contrast to the single ITIM observed for human KIR2DL4. In addition, soluble HLA-G is identified as KIR2DL4 ligand in humans, but in macaques the gene encoding this non-classical MHC diverged into a pseudogene. However, a similar gene, Mamu-AG, has been identified that is expressed on trophoblasts and might facilitate interactions with macaque KIR2DL04, although binding is not 
demonstrated [167]. A framework gene that marks the other side of the telomeric KIR haplotype in macaques has not been reported, but is most likely a lineage II KIR gene.

In 2005, and more recently in 2019, BAC clones that covered complete rhesus macaque KIR haplotypes were completely sequenced and constructed [159, 168]. These two haplotypes demonstrated that the physical location of KIR3DL20 resembles the KIR3DL3 locus in humans, substantiating that these genes share a common progenitor. KIR1D has been identified next to the KIR3DL20. Although all 9 exons are identified for KIR1D at the genomic level, exons 3 and 5, which contain deletions that would shift the reading frame, are not incorporated at the transcription level. In addition, a pseudogene, KIR2DLO4, and lineage II KIR genes are documented on these relatively short $K I R$ haplotypes. In contrast to humans, chimpanzees, and orangutans, the macaque $K I R$ genes are expanded in the telomeric region, whereas the centromeric region may only contain KIR3DL20, KIR1D, and a putative pseudogene. Although informative, these two haplotypes do not provide complete information on the haplotype configurations in macaques.

Most studies were performed in Indian rhesus macaques, whereas only a few individuals from the Chinese and Burmese populations are genomically characterized for their KIR repertoire [166, 169-175]. Less well characterized is the KIR gene repertoire in cynomolgus macaques. Only the isolated and relatively recently established Mauritian population is analyzed for their KIR gene content and shows limited variation due to the small number of founders $[176,177]$. Despite that the majority of the macaque studies involved the characterization of $K I R$ genotypes and haplotypes by determining the presence or absence of $K I R$ genes, species-specific variation is documented for both macaque species. A handful of studies involved the transcriptomic characterization by Sanger sequencing and illustrated extensive allelic polymorphism and putative recombinant genes.

\section{Evolution of sequencing techniques}

Over the years, sequencing techniques have been improved, which enables a higher throughput for the characterization of complex genetic regions, like the KIR gene region. Up to now, however, only the conventional sequencing approaches have been used for the macaque KIR gene cluster, although new techniques are available that might improve the characterization.

It was in 1953 that Watson and Crick solved the structure of DNA [178], which is composed of four deoxyribonucleic acids (adenine, cytosine, thymine, guanine). Upon this discovery, it took over 20 years to develop sufficient techniques that were able to decode the DNA, which harbors the essential information about our phylogeny, ancestry, and susceptibility to disease. Early attempts to sequence DNA involved twodimensional separation of radioactively labeled DNA fragments and reported 24 bases of a lactose-repressor binding site, which took two years [179]. In around 1976, the 
development of first-generation sequencing techniques accelerated with approaches reported by Sanger and Gilbert, which enabled the separation of DNA fragments on a polyacrylamide gel at a single-base resolution [180, 181]. Especially Sanger's sequencing approach was improved over the years by the introduction of enhanced polymerases and chemicals, and this led to the release of the first fluorescent-based sequencing machines in 1987, which enabled the sequencing of 1.000 bases per day [182]. These improvements paved to way for the Human Genome Project (HGP) that started in 1990 and aimed to sequence the complete human genome. Propelled by a string of breakthroughs, the efforts of labs around the world, and billions of dollars, the human genome sequence was completed in 2004 [183]. One year later, secondgeneration sequencing instruments became commercially available, such as the 454 (Roche), the Solexa (Illumina), and the lonTorrent (Rothberg), the fast developments of which enhanced the quality and quantity of sequenced DNA and lowered the costs. To illustrate, the number of bases that could be sequenced within two days by a secondgeneration sequencing approach exceeds the size of the HGP by a factor of 40 . Nowadays, the Illumina platform is the only second-generation technique that is still widely used and supported. Third-generation sequencing platforms, which enable real-time and single-molecule (SMRT) sequencing, include Pacific Biosciences (PacBio) and Oxford Nanopore sequencing. The PacBio platform measures the incorporation of a single fluorescently labelled nucleotide into a growing DNA chain using a zero-mode waveguide, and enables relatively high accuracy sequencing of long reads (10-100 kb) $[184,185]$. The Nanopore platform, of which the technique was already hypothesized in the 1990s [186], pulls complete strains of DNA through a narrow pore. This causes shifts in ion currents that reveals the sequence of the DNA [187]. The highest readlengths that are currently obtained by Nanopore sequencing goes up to $900 \mathrm{~kb}$, but the relatively low accuracy requires correction by a high coverage. A major advantage of the Nanopore platform is the portability of the pocket-sized device. The two thirdgeneration sequencing platforms do not only enable a high sequencing throughput of large DNA fragments, which allows the sequencing of a complete human genome within two days at the cost of approximately thousand dollars, but are also capable of DNA modification sequencing, such as methylation (Table IV) [188-190].

The efforts made in the last half century resulted in the development and improvement of sequencing approaches, which are still not plateaued in accuracy, throughput and cost. By combining the golden standard of Sanger sequencing with both third-generation sequencing platforms, PacBio and Nanopore, complex immunogenic regions in the macaque genome, such as the KIR gene cluster that exceeds the complexity of similar regions in humans, can be elucidated. 
Table IV. Comparison of Sanger sequencing and third-generation sequencing platforms. Table is adapted from [190].

\begin{tabular}{lllllll}
\hline Technique & Platform & Cost & Avg. Output (Gb) & Avg./Max. read length & Run time & Error rate \\
\hline Sanger & - & $\$$ & 0.00006 & $650 \mathrm{bp}$ & $0.5-1 \mathrm{~h}$ & $0.1 \%$ \\
\hline \multirow{2}{*}{ PacBio } & RS II & $\$ \$ \$$ & 2 & $5-15 \mathrm{~kb} />60 \mathrm{~kb}$ & $0.5-6 \mathrm{~h}$ & $10-15 \%$ \\
\cline { 2 - 7 } & Sequel & $\$ \$ \$$ & $8-10$ & $5-30 \mathrm{~kb} />200 \mathrm{~kb}$ & $0.5-20 \mathrm{~h}$ & $10-15 \%$ \\
\cline { 2 - 7 } & Sequel II & $\$ \$$ & $56-70$ & $5-30 \mathrm{~kb} />200 \mathrm{~kb}$ & $0.5-30 \mathrm{~h}$ & $10-15 \%$ \\
\hline \multirow{3}{*}{ Nanopore } & Flongle & $\$$ & 1 & $5-35 \mathrm{~kb} />2 \mathrm{Mb}$ & $0.5-48 \mathrm{~h}$ & $5-15 \%$ \\
\cline { 2 - 7 } & MinION & $\$$ & 15 & $5-35 \mathrm{~kb} />2 \mathrm{Mb}$ & $0.5-48 \mathrm{~h}$ & $5-15 \%$ \\
\cline { 2 - 7 } & GridION & $\$ \$$ & 75 & $5-35 \mathrm{~kb} />2 \mathrm{Mb}$ & $0.5-48 \mathrm{~h}$ & $5-15 \%$ \\
\cline { 2 - 7 } & PromethION & $\$ \$$ & $4-6 \mathrm{~Tb}$ & $5-35 \mathrm{~kb} />2 \mathrm{Mb}$ & $0.5-72 \mathrm{~h}$ & $5-15 \%$ \\
\hline
\end{tabular}

\section{Thesis aim and overview}

This manuscript is aimed to provide more insights into the KIR gene system of macaques. Previous characterization studies already hinted on the complexity of this system, but these experiments relied on conventional sequencing techniques, such as Sanger sequencing and Roche/454 pyrosequencing, or on the determination of the presence or absence of KIR genes at the genomic DNA level. Although these studies were informative, a comprehensive overview on the macaque $K I R$ gene system is still lacking. Furthermore, it has been demonstrated that the geographical origin of macaques that are used as biomedical model influences experimental outcomes, which is most probably related to immunogenetic differences. An improved characterization of the macaque KIR genes might refine the selection of animals that are used for biomedical studies based on genetic knowledge in different macaque species, populations and individuals. Such an approach has already shown beneficial in case of the MHC genes [191, 192]. In addition, this manuscript is intended to provide a comparison of the macaque and human KIR gene clusters, and how these clusters relate with previous published data on the KIR genes in other primate species. The immunogenetic comparison might elucidate differential and conserved characteristics in the KIR gene evolution in different species, which further the understanding on receptor function, ligands, and evolutionary selective forces. At last, novel methods, techniques, and sequencing platforms that are described in this manuscript to characterize the highly complex macaque KIR gene system can be applied for the characterization of plastic multigenic regions in other species, including humans.

The sequencing of macaque and human KIR genes was mainly performed by Sanger sequencing, or by the profiling of KIR genes at the genomic DNA level, which are time 
consuming methods or do not provide allele level resolution. Allele level specificity and functionality is, however, documented for multiple KIR and might be of importance to interpret disease association studies. Therefore, a method was established that enabled a high-throughput characterization of both macaque and human KIR gene transcripts by sequencing on a PacBio platform. Abundant novel KIR alleles and gene entities were confirmed in these transcriptome datasets. Even more, multiple recombinant $K I R$ genes were identified that would be missed by most conventional sequencing techniques. Transcriptomic macaque and human KIR haplotypes were subsequently defined by segregation studies, which illustrated the plasticity of the macaque KIR gene organization, and confirmed novel and previously reported human haplotype configurations (Chapter 2).

The divergence of the human and macaque KIR genes is in line with the evolutionary distance to their common ancestor that lived approximately 25 million years ago. There is, however, also significant diversity reported in the closely related rhesus and cynomolgus macaque species that diverged only 1-3 million years ago, which indicates an unparalleled rapid evolution of the macaque $K I R$ gene system. This is further substantiated by allele and gene level diversity in different geographical macaque populations, such as the Indian, Chinese, and Burmese rhesus macaques, and the mainland, insular, and Mauritian cynomolgus macaques (Chapter 3). The KIR gene characterization of the cynomolgus macaques and the large number of recombinant $K I R$ genes also stressed the need for a revised macaque KIR nomenclature report [62].

Allelic polymorphism, recombinations, and copy number variations contribute to the diversity of the KIR genes, but less well defined is the variation that is generated by alternative splicing. The impact of this posttranscriptional process is evident for the KIR genes in both macaques and humans, as abundant exon skipping events, alternative splice sites, and cryptic exons are described. These isoforms might differ in their expression status, function, cellular localization, and binding properties, and thereby significantly contribute to the variation of the KIR gene system (Chapter 4).

Whereas human KIR haplotypes mostly follow standard configurations with some differential recombinations, the knowledge on macaque KIR haplotypes is restricted to two completely sequenced rhesus macaque haplotypes. Macaque KIR haplotypes that were deduced from transcriptome studies have shown variable gene content and a less strict framework, but the exact configuration remains elusive. The combination of targeted enrichment using Cas9 nuclease followed by sequencing on an Oxford Nanopore MinION platform, enabled the construction of complete macaque KIR haplotypes at an allele level resolution. This technique was further validated by the assembly of human KIR haplotypes. Overall, the targeted enrichment and long-read sequencing provides a cost- and time-efficient method to characterize complete haplotypes of complex multigenic regions in different species (Chapter 5).

In conclusion, a comprehensive overview of the macaque KIR gene region is provided that include a summary and discussion on the extensive diversity that has been reported in rhesus and cynomolgus macaques. Furthermore, a comparison of the $K I R$ cluster in other primate species illustrates the differences and similarities that 
have emerged during the rapid evolution of the KIR genes, and shows the relevance for non-human primate models in translatable biomedical research (Chapter 6).

With the new sequencing techniques available, the genetic organization of the KIR gene cluster in macaque species is more and more elucidated. These insights pave the way to examine the role of KIR in different biological processes (Chapter 7). The expression of KIR on tissue-resident NK cells, for instance, might display more specialized functions. Even more, a highly regulated alternative splicing machinery might generate specific KIR isoforms during different processes. As such, the functional impact of the broad KIR receptor diversity might stretch beyond the current findings and assumptions. 


\section{References}

1. Groves C: Species concept in primates. American journal of primatology 2012, 74(8):687-691.

2. Bassing CH, Swat W, Alt FW: The Mechanism and Regulation of Chromosomal V(D)J Recombination. Cell 2002, 109(2, Supplement 1):S45-S55.

3. Hazenberg MD, Spits H: Human innate lymphoid cells. Blood 2014, 124(5):700-709.

4. Kiessling R, Klein E, Pross H, Wigzell H: "Natural" killer cells in the mouse. II. Cytotoxic cells with specificity for mouse Moloney leukemia cells. Characteristics of the killer cell. Eur J Immunol 1975, 5(2):117-121.

5. Takasugi M, Mickey MR, Terasaki PI: Reactivity of lymphocytes from normal persons on cultured tumor cells. Cancer research 1973, 33(11):2898-2902.

6. Herberman RB, Nunn ME, Holden HT, Lavrin DH: Natural cytotoxic reactivity of mouse lymphoid cells against syngeneic and allogeneic tumors. II. Characterization of effector cells. International journal of cancer 1975, 16(2):230-239.

7. Kim S, lizuka K, Aguila HL, Weissman IL, Yokoyama WM: In vivo natural killer cell activities revealed by natural killer cell-deficient mice. Proceedings of the National Academy of Sciences of the United States of America 2000, 97(6):2731-2736.

8. Smyth MJ, Cretney E, Kelly JM, Westwood JA, Street SE, Yagita H, Takeda K, van Dommelen SL, Degli-Esposti MA, Hayakawa Y: Activation of NK cell cytotoxicity. Molecular immunology 2005, 42(4):501-510.

9. Tripp CS, Wolf SF, Unanue ER: Interleukin 12 and tumor necrosis factor alpha are costimulators of interferon gamma production by natural killer cells in severe combined immunodeficiency mice with listeriosis, and interleukin 10 is a physiologic antagonist. Proceedings of the National Academy of Sciences of the United States of America 1993, 90(8):3725-3729.

10. Sun JC, Beilke JN, Lanier LL: Adaptive immune features of natural killer cells. Nature 2009, 457(7229):557-561.

11. Guma M, Angulo A, Vilches C, Gomez-Lozano N, Malats N, Lopez-Botet M: Imprint of human cytomegalovirus infection on the NK cell receptor repertoire. Blood 2004, 104(12):3664-3671.

12. Fauriat C, Long EO, Ljunggren HG, Bryceson YT: Regulation of human NK-cell cytokine and chemokine production by target cell recognition. Blood 2010, 115(11):2167-2176.

13. De Maria A, Bozzano F, Cantoni C, Moretta L: Revisiting human natural killer cell subset function revealed cytolytic CD56(dim)CD16+ NK cells as rapid producers of abundant IFN-gamma on activation. Proceedings of the National Academy of Sciences of the United States of America 2011, 108(2):728-732.

14. Dulphy N, Haas P, Busson M, Belhadj S, Peffault de Latour R, Robin M, Carmagnat M, Loiseau P, Tamouza R, Scieux C et al: An unusual CD56(bright) CD16(low) NK cell subset dominates the early posttransplant period following HLA-matched hematopoietic stem cell transplantation. Journal of immunology (Baltimore, Md : 1950) 2008, 181(3):2227-2237.

15. Yu J, Mao HC, Wei M, Hughes T, Zhang J, Park I-k, Liu S, McClory S, Marcucci G, Trotta R et al: CD94 surface density identifies a functional intermediary between the CD56bright and CD56dim human NK-cell subsets. Blood 2010, 115(2):274-281.

16. Chan A, Hong DL, Atzberger A, Kollnberger S, Filer AD, Buckley CD, McMichael A, Enver T, Bowness $P$ : CD56bright human NK cells differentiate into CD56dim cells: role of contact with peripheral fibroblasts. Journal of immunology (Baltimore, Md : 1950) 2007, 179(1):89-94.

17. Romagnani C, Juelke K, Falco M, Morandi B, D’Agostino A, Costa R, Ratto G, Forte G, Carrega P, Lui G et al: CD56brightCD16- killer Ig-like receptor- NK cells display longer telomeres and acquire features of CD56dim NK cells upon activation. Journal of immunology (Baltimore, Md : 1950) 2007, 178(8):4947-4955.

18. Nguyen S, Dhedin N, Vernant JP, Kuentz M, Al Jijakli A, Rouas-Freiss N, Carosella ED, Boudifa A, Debre P, Vieillard $\mathrm{V}$ : NK-cell reconstitution after haploidentical hematopoietic stem-cell transplantations: immaturity of NK cells and inhibitory effect of NKG2A override GvL effect. Blood 2005, 105(10):4135-4142.

19. Andrews DM, Smyth MJ: A potential role for RAG-1 in NK cell development revealed by analysis of NK cells during ontogeny. Immunology and cell biology 2010, 88(2):107-116.

20. Freud AG, Becknell B, Roychowdhury S, Mao HC, Ferketich AK, Nuovo GJ, Hughes TL, Marburger TB, Sung J, Baiocchi RA et al: A human CD34(+) subset resides in lymph nodes and differentiates into CD56bright natural killer cells. Immunity 2005, 22(3):295-304. 
21. Kim S, lizuka K, Kang HS, Dokun A, French AR, Greco S, Yokoyama WM: In vivo developmental stages in murine natural killer cell maturation. Nature immunology 2002, 3(6):523-528.

22. Santoni A, Carlino C, Gismondi A: Uterine NK cell development, migration and function. Reproductive biomedicine online 2008, 16(2):202-210.

23. Ferlazzo G, Thomas D, Lin SL, Goodman K, Morandi B, Muller WA, Moretta A, Munz C: The abundant NK cells in human secondary lymphoid tissues require activation to express killer cell lg-like receptors and become cytolytic. Journal of immunology (Baltimore, Md : 1950) 2004, 172(3):1455-1462.

24. Dalbeth N, Gundle R, Davies RJ, Lee YC, McMichael AJ, Callan MF: CD56bright NK cells are enriched at inflammatory sites and can engage with monocytes in a reciprocal program of activation. Journal of immunology (Baltimore, Md : 1950) 2004, 173(10):6418-6426.

25. Shiow LR, Rosen DB, Brdickova N, Xu Y, An J, Lanier LL, Cyster JG, Matloubian M: CD69 acts downstream of interferon-alpha/beta to inhibit S1P1 and lymphocyte egress from lymphoid organs. Nature 2006, 440(7083):540-544.

26. Jenne CN, Enders A, Rivera R, Watson SR, Bankovich AJ, Pereira JP, Xu Y, Roots CM, Beilke JN, Banerjee A et al: T-bet-dependent S1P5 expression in NK cells promotes egress from lymph nodes and bone marrow. The Journal of experimental medicine 2009, 206(11):2469-2481.

27. Campbell JJ, Qin S, Unutmaz D, Soler D, Murphy KE, Hodge MR, Wu L, Butcher EC: Unique subpopulations of CD56+ NK and NK-T peripheral blood lymphocytes identified by chemokine receptor expression repertoire. Journal of immunology (Baltimore, Md : 1950) 2001, 166(11):6477-6482.

28. Melsen JE, Lugthart G, Lankester AC, Schilham MW: Human Circulating and Tissue-Resident CD56(bright) Natural Killer Cell Populations. Front Immunol 2016, 7:262-262.

29. Sun JC, Beilke JN, Lanier LL: Adaptive immune features of natural killer cells. Nature 2009, 457(7229):557-561.

30. Halenius A, Gerke C, Hengel H: Classical and non-classical MHC I molecule manipulation by human cytomegalovirus: so many targets-but how many arrows in the quiver? Cellular \& molecular immunology 2015 12(2):139-153.

31. Schlums H, Cichocki F, Tesi B, Theorell J, Beziat V, Holmes TD, Han H, Chiang SC, Foley B, Mattsson K et al: Cytomegalovirus infection drives adaptive epigenetic diversification of NK cells with altered signaling and effector function. Immunity 2015, 42(3):443-456.

32. Grzywacz B, Kataria N, Kataria N, Blazar BR, Miller JS, Verneris MR: Natural killer-cell differentiation by myeloid progenitors. Blood 2011, 117(13):3548-3558.

33. Chen Q, Ye W, Jian Tan W, Mei Yong KS, Liu M, Qi Tan S, Loh E, Te Chang K, Chye Tan T, Preiser PR et al: Delineation of Natural Killer Cell Differentiation from Myeloid Progenitors in Human. Scientific Reports 2015, 5:15118.

34. Wu C, Espinoza DA, Koelle SJ, Yang D, Truitt L, Schlums H, Lafont BA, Davidson-Moncada JK, Lu R, Kaur A et al: Clonal expansion and compartmentalized maintenance of rhesus macaque NK cell subsets. Science immunology 2018, 3(29).

35. Wu C, Li B, Lu R, Koelle SJ, Yang Y, Jares A, Krouse AE, Metzger M, Liang F, Lore K et al: Clonal tracking of rhesus macaque hematopoiesis highlights a distinct lineage origin for natural killer cells. Cell stem cell 2014, 14(4):486499.

36. Elliott JM, Wahle JA, Yokoyama WM: MHC class I-deficient natural killer cells acquire a licensed phenotype after transfer into an MHC class I-sufficient environment. The Journal of experimental medicine 2010, 207(10):20732079 .

37. Joncker NT, Shifrin N, Delebecque F, Raulet DH: Mature natural killer cells reset their responsiveness when exposed to an altered MHC environment. The Journal of experimental medicine 2010, 207(10):2065-2072.

38. Kim S, Poursine-Laurent J, Truscott SM, Lybarger L, Song YJ, Yang L, French AR, Sunwoo JB, Lemieux S, Hansen TH et al: Licensing of natural killer cells by host major histocompatibility complex class I molecules. Nature 2005, 436(7051):709-713.

39. Bix M, Liao NS, Zijlstra M, Loring J, Jaenisch R, Raulet D: Rejection of class I MHC-deficient haemopoietic cells by irradiated MHC-matched mice. Nature 1991, 349(6307):329-331.

40. Liao NS, Bix M, Zijlstra M, Jaenisch R, Raulet D: MHC class I deficiency: susceptibility to natural killer (NK) cells and impaired NK activity. Science (New York, NY) 1991, 253(5016):199-202. 
41. Belanger S, Tu MM, Rahim MM, Mahmoud AB, Patel R, Tai LH, Troke AD, Wilhelm BT, Landry JR, Zhu Q et al: Impaired natural killer cell self-education and "missing-self" responses in Ly49-deficient mice. Blood 2012, 120(3):592-602.

42. Joncker NT, Fernandez NC, Treiner E, Vivier E, Raulet DH: NK cell responsiveness is tuned commensurate with the number of inhibitory receptors for self-MHC class I: the rheostat model. Journal of immunology (Baltimore, Md : 1950) 2009, 182(8):4572-4580.

43. Restifo NP, Esquivel F, Kawakami Y, Yewdell JW, Mule JJ, Rosenberg SA, Bennink JR: Identification of human cancers deficient in antigen processing. The Journal of experimental medicine 1993, 177(2):265-272.

44. Restifo NP, Marincola FM, Kawakami Y, Taubenberger J, Yannelli JR, Rosenberg SA: Loss of functional beta 2-microglobulin in metastatic melanomas from five patients receiving immunotherapy. Journal of the National Cancer Institute 1996, 88(2):100-108.

45. Collins KL, Chen BK, Kalams SA, Walker BD, Baltimore D: HIV-1 Nef protein protects infected primary cells against killing by cytotoxic T lymphocytes. Nature 1998, 391(6665):397-401.

46. Ljunggren HG, Karre K: In search of the 'missing self': MHC molecules and NK cell recognition. Immunology today 1990, 11(7):237-244.

47. Tarek N, Le Luduec JB, Gallagher MM, Zheng J, Venstrom JM, Chamberlain E, Modak S, Heller G, Dupont B, Cheung NK et al: Unlicensed NK cells target neuroblastoma following anti-GD2 antibody treatment. The Journal of clinical investigation 2012, 122(9):3260-3270.

48. Tu MM, Mahmoud AB, Makrigiannis AP: Licensed and Unlicensed NK Cells: Differential Roles in Cancer and Viral Control. Front Immunol 2016, 7:166.

49. Orr MT, Murphy WJ, Lanier LL: 'Unlicensed' natural killer cells dominate the response to cytomegalovirus infection. Nature immunology 2010, 11(4):321-327.

50. Barrow AD, Martin CJ, Colonna M: The Natural Cytotoxicity Receptors in Health and Disease. Front Immunol 2019, 10(909).

51. Braud VM, Allan DS, O'Callaghan CA, Soderstrom K, D'Andrea A, Ogg GS, Lazetic S, Young NT, Bell JI, Phillips JH et al: HLA-E binds to natural killer cell receptors CD94/NKG2A, B and C. Nature 1998, 391(6669):795-799.

52. Lopez-Larrea C, Suarez-Alvarez B, Lopez-Soto A, Lopez-Vazquez A, Gonzalez S: The NKG2D receptor: sensing stressed cells. Trends in molecular medicine 2008, 14(4):179-189.

53. Storkus WJ, Alexander J, Payne JA, Dawson JR, Cresswell P: Reversal of natural killing susceptibility in target cells expressing transfected class I HLA genes. Proceedings of the National Academy of Sciences of the United States of America 1989, 86(7):2361-2364.

54. Shimizu Y, DeMars R: Demonstration by class I gene transfer that reduced susceptibility of human cells to natural killer cell-mediated lysis is inversely correlated with HLA class I antigen expression. Eur J Immunol 1989, 19(3):447-451.

55. Colonna M, Samaridis J: Cloning of immunoglobulin-superfamily members associated with HLA-C and HLA-B recognition by human natural killer cells. Science (New York, NY) 1995, 268(5209):405-408.

56. Wagtmann N, Biassoni R, Cantoni C, Verdiani S, Malnati MS, Vitale M, Bottino C, Moretta L, Moretta A, Long EO: Molecular clones of the p58 NK cell receptor reveal immunoglobulin-related molecules with diversity in both the extra- and intracellular domains. Immunity 1995, 2(5):439-449.

57. D'Andrea A, Chang C, Franz-Bacon K, McClanahan T, Phillips JH, Lanier LL: Molecular cloning of NKB1. A natural killer cell receptor for HLA-B allotypes. Journal of immunology (Baltimore, Md : 1950) 1995, 155(5):2306-2310.

58. Long EO, Colonna M, Lanier LL: Inhibitory MHC class I receptors on NK and T cells: a standard nomenclature. Immunology today 1996, 17(2):100.

59. Andre P, Biassoni R, Colonna M, Cosman D, Lanier LL, Long EO, Lopez-Botet M, Moretta A, Moretta L, Parham P et al: New nomenclature for MHC receptors. Nature immunology 2001, 2(8):661.

60. Marsh SG, Parham P, Dupont B, Geraghty DE, Trowsdale J, Middleton D, Vilches C, Carrington M, Witt C, Guethlein LA et al: Killer-cell immunoglobulin-like receptor (KIR) nomenclature report, 2002. Human immunology 2003, 64(6):648-654.

61. Robinson J, Guethlein LA, Maccari G, Blokhuis J, Bimber BN, de Groot NG, Sanderson ND, Abi-Rached L, Walter L, Bontrop RE et al: Nomenclature for the KIR of non-human species. Immunogenetics 2018, 70(9):571-583. 
62. Bruijnesteijn J, de Groot NG, Otting N, Maccari G, Guethlein LA, Robinson J, Marsh SGE, Walter L, O'Connor DH, Hammond JA et al: Nomenclature report for killer-cell immunoglobulin-like receptors (KIR) in macaque species: new genes/alleles, renaming recombinant entities and IPD-NHKIR updates. Immunogenetics 2019.

63. Trowsdale J, Jones DC, Barrow AD, Traherne JA: Surveillance of cell and tissue perturbation by receptors in the LRC. Immunological reviews 2015, 267(1):117-136.

64. Wende H, Colonna M, Ziegler A, Volz A: Organization of the leukocyte receptor cluster (LRC) on human chromosome 19q13.4. Mammalian genome : official journal of the International Mammalian Genome Society 1999, 10(2):154-160.

65. Barrow AD, Trowsdale J: The extended human leukocyte receptor complex: diverse ways of modulating immune responses. Immunological reviews 2008, 224:98-123.

66. Guethlein LA, Abi-Rached L, Hammond JA, Parham P: The expanded cattle KIR genes are orthologous to the conserved single-copy KIR3DX1 gene of primates. Immunogenetics 2007, 59(6):517-522.

67. Sanderson ND, Norman PJ, Guethlein LA, Ellis SA, Williams C, Breen M, Park SD, Magee DA, Babrzadeh F, Warry A et al: Definition of the cattle killer cell Ig-like receptor gene family: comparison with aurochs and human counterparts. Journal of immunology (Baltimore, Md : 1950) 2014, 193(12):6016-6030.

68. Moradi S, Berry R, Pymm P, Hitchen C, Beckham SA, Wilce MC, Walpole NG, Clements CS, Reid HH, Perugini MA et al: The structure of the atypical killer cell immunoglobulin-like receptor, KIR2DL4. The Journal of biological chemistry 2015, 290(16):10460-10471.

69. Rajagopalan S, Long EO: KIR2DL4 (CD158d): An activation receptor for HLA-G. Front Immunol 2012, 3:258-258.

70. Faure M, Long EO: KIR2DL4 (CD158d), an NK Cell-Activating Receptor with Inhibitory Potential. The Journal of Immunology 2002, 168(12):6208.

71. Trundley AE, Hiby SE, Chang C, Sharkey AM, Santourlidis S, Uhrberg M, Trowsdale J, Moffett A: Molecular characterization of KIR3DL3. Immunogenetics 2006, 57(12):904-916.

72. Trompeter HI, Gomez-Lozano N, Santourlidis S, Eisermann B, Wernet P, Vilches C, Uhrberg M: Three structurally and functionally divergent kinds of promoters regulate expression of clonally distributed killer cell Ig-like receptors (KIR), of KIR2DL4, and of KIR3DL3. Journal of immunology (Baltimore, Md : 1950) 2005, 174(7):41354143.

73. Leaton LA, Shortt J, Kichula KM, Tao S, Nemat-Gorgani N, Mentzer AJ, Oppenheimer SJ, Deng Z, Hollenbach JA, Gignoux CR et al: Conservation, Extensive Heterozygosity, and Convergence of Signaling Potential All Indicate a Critical Role for KIR3DL3 in Higher Primates. Front Immunol 2019, 10:24.

74. Nutalai R, Gaudieri S, Jumnainsong A, Leelayuwat C: Regulation of KIR3DL3 Expression via Mirna. Genes 2019, 10(8).

75. Hollenbach JA, Nocedal I, Ladner MB, Single RM, Trachtenberg EA: Killer cell immunoglobulin-like receptor (KIR) gene content variation in the HGDP-CEPH populations. Immunogenetics 2012, 64(10):719-737.

76. Hilton HG, Guethlein LA, Goyos A, Nemat-Gorgani N, Bushnell DA, Norman PJ, Parham P: Polymorphic HLA-C Receptors Balance the Functional Characteristics of KIR Haplotypes. Journal of immunology (Baltimore, Md: 1950) 2015, 195(7):3160-3170.

77. Rajagopalan S, Long EO: KIR2DL4 (CD158d): An activation receptor for HLA-G. Front Immunol 2012, 3:258.

78. Garcia-Beltran WF, Hölzemer A, Martrus G, Chung AW, Pacheco Y, Simoneau CR, Rucevic M, Lamothe-Molina PA, Pertel T, Kim T-E et al: Open conformers of HLA-F are high-affinity ligands of the activating NK-cell receptor KIR3DS1. Nature immunology 2016, 17(9):1067-1074.

79. Graef T, Moesta AK, Norman PJ, Abi-Rached L, Vago L, Older Aguilar AM, Gleimer M, Hammond JA, Guethlein LA, Bushnell DA et al: KIR2DS4 is a product of gene conversion with KIR3DL2 that introduced specificity for HLA-A*11 while diminishing avidity for HLA-C. The Journal of experimental medicine 2009, 206(11):2557-2572

80. Della Chiesa M, Romeo E, Falco M, Balsamo M, Augugliaro R, Moretta L, Bottino C, Moretta A, Vitale M: Evidence that the KIR2DS5 gene codes for a surface receptor triggering natural killer cell function. Eur J Immunol 2008, 38(8):2284-2289.

81. Katz G, Markel G, Mizrahi S, Arnon TI, Mandelboim O: Recognition of HLA-Cw4 but not HLA-Cw6 by the NK cell receptor killer cell Ig-like receptor two-domain short tail number 4. Journal of immunology (Baltimore, Md: 1950) 2001, 166(12):7260-7267. 
82. Liu J, Xiao Z, Ko HL, Shen M, Ren EC: Activating killer cell immunoglobulin-like receptor 2DS2 binds to HLA-A*11. Proceedings of the National Academy of Sciences of the United States of America 2014, 111(7):2662-2667.

83. Colonna M, Spies T, Strominger JL, Ciccone E, Moretta A, Moretta L, Pende D, Viale O: Alloantigen recognition by two human natural killer cell clones is associated with HLA-C or a closely linked gene. Proceedings of the National Academy of Sciences of the United States of America 1992, 89(17):7983-7985.

84. Moesta AK, Norman PJ, Yawata M, Yawata N, Gleimer M, Parham P: Synergistic polymorphism at two positions distal to the ligand-binding site makes KIR2DL2 a stronger receptor for HLA-C than KIR2DL3. Journal of immunology (Baltimore, Md: 1950) 2008, 180(6):3969-3979.

85. Parham P: MHC class I molecules and kirs in human history, health and survival. Nature Reviews Immunology 2005, 5(3):201-214.

86. Winter CC, Gumperz JE, Parham P, Long EO, Wagtmann N: Direct binding and functional transfer of NK cell inhibitory receptors reveal novel patterns of HLA-C allotype recognition. Journal of immunology (Baltimore, Md : 1950) 1998, 161(2):571-577.

87. Wagtmann N, Rajagopalan S, Winter CC, Peruzzi M, Long EO: Killer cell inhibitory receptors specific for HLA-C and HLA-B identified by direct binding and by functional transfer. Immunity 1995, 3(6):801-809.

88. Vilches C, Rajalingam R, Uhrberg M, Gardiner CM, Young NT, Parham P: KIR2DL5, a novel killer-cell receptor with a DO-D2 configuration of Ig-like domains. Journal of immunology (Baltimore, Md : 1950) 2000, 164(11):5797-5804.

89. Hilton HG, Blokhuis JH, Guethlein LA, Norman PJ, Parham P: Resurrecting KIR2DP1: A Key Intermediate in the Evolution of Human Inhibitory NK Cell Receptors That Recognize HLA-C. Journal of immunology (Baltimore, Md : 1950) 2017, 198(5):1961-1973.

90. Bono M, Pende D, Bertaina A, Moretta A, Della Chiesa M, Sivori S, Zecca M, Locatelli F, Moretta L, Bottino C et al: Analysis of \&lt;em\&gt;KIR3DP1\&lt;/em\&gt; Polymorphism Provides Relevant Information on Centromeric \&lt;em\&gt;KIR\&It;/em\&gt; Gene Content. The Journal of Immunology 2018:ji1800564.

91. Shaw J, Kollnberger S: New perspectives on the ligands and function of the killer cell immunoglobulin-like receptor KIR3DL2 in health and disease. Front Immunol 2012, 3(339).

92. Chapel A, Garcia-Beltran WF, Hölzemer A, Ziegler M, Lunemann S, Martrus G, Altfeld M: Peptide-specific engagement of the activating NK cell receptor KIR2DS1. Scientific Reports 2017, 7(1):2414.

93. Parham P, Norman PJ, Abi-Rached L, Guethlein LA: Human-specific evolution of killer cell immunoglobulin-like receptor recognition of major histocompatibility complex class I molecules. Philosophical transactions of the Royal Society of London Series B, Biological sciences 2012, 367(1590):800-811.

94. Older Aguilar AM, Guethlein LA, Adams EJ, Abi-Rached L, Moesta AK, Parham P: Coevolution of killer cell Ig-like receptors with HLA-C to become the major variable regulators of human NK cells. Journal of immunology (Baltimore, Md : 1950) 2010, 185(7):4238-4251.

95. Hilton HG, Norman PJ, Nemat-Gorgani N, Goyos A, Hollenbach JA, Henn BM, Gignoux CR, Guethlein LA, Parham $P$ : Loss and Gain of Natural Killer Cell Receptor Function in an African Hunter-Gatherer Population. PLOS genetics 2015, 11(8):e1005439.

96. Cassidy SA, Cheent KS, Khakoo SI: Effects of Peptide on NK cell-mediated MHCI recognition. Front Immunol 2014, 5:133.

97. Cassidy S, Mukherjee S, Myint TM, Mbiribindi B, North H, Traherne J, Mulder A, Claas FH, Purbhoo MA, Das J et al: Peptide selectivity discriminates NK cells from KIR2DL2- and KIR2DL3-positive individuals. European journal of immunology 2015, 45(2):492-500.

98. Brusilovsky M, Cordoba M, Rosental B, Hershkovitz O, Andrake MD, Pecherskaya A, Einarson MB, Zhou Y, Braiman A, Campbell KS et al: Genome-wide siRNA screen reveals a new cellular partner of NK cell receptor KIR2DL4: heparan sulfate directly modulates KIR2DL4-mediated responses. Journal of immunology (Baltimore, Md : 1950) 2013, 191(10):5256-5267.

99. Mele D, Pasi A, Cacciatore R, Mantovani S, Oliviero B, Mondelli MU, Varchetta S: Decreased interferon- $\gamma$ production by NK cells from KIR haplotype B carriers in hepatitis C virus infection. Liver International 2019, 39(7):1237-1245.

100. Wauquier N, Padilla C, Becquart P, Leroy E, Vieillard V: Association of KIR2DS1 and KIR2DS3 with fatal outcome in Ebola virus infection. Immunogenetics 2010, 62(11):767-771. 
101. Aranda-Romo S, Garcia-Sepulveda CA, Comas-García A, Lovato-Salas F, Salgado-Bustamante M, Gómez-Gómez A, Noyola DE: Killer-cell immunoglobulin-like receptors (KIR) in severe A (H1N1) 2009 influenza infections. Immunogenetics 2012, 64(9):653-662.

102. Martin MP, Qi Y, Gao X, Yamada E, Martin JN, Pereyra F, Colombo S, Brown EE, Shupert WL, Phair J et al: Innate partnership of HLA-B and KIR3DL1 subtypes against HIV-1. Nature genetics 2007, 39(6):733-740.

103. Pelak K, Need AC, Fellay J, Shianna KV, Feng S, Urban TJ, Ge D, De Luca A, Martinez-Picado J, Wolinsky SM et al: Copy number variation of KIR genes influences HIV-1 control. PLoS Biol 2011, 9(11):e1001208-e1001208.

104. Chaisri S, Pabalan N, Tabunhan S, Tharabenjasin P, Sankuntaw N, Leelayuwat C: Effects of the killer immunoglobulin-like receptor (KIR) polymorphisms on HIV acquisition: A meta-analysis. PloS one 2019, 14(12):e0225151.

105. Leone P, De Re V, Vacca A, Dammacco F, Racanelli V: Cancer treatment and the KIR-HLA system: an overview. Clinical and experimental medicine 2017, 17(4):419-429.

106. He Y, Bunn PA, Zhou C, Chan D: KIR 2D (L1, L3, L4, S4) and KIR 3DL1 protein expression in non-small cell lung cancer. Oncotarget 2016, 7(50):82104-82111.

107. Vineretsky KA, Karagas MR, Christensen BC, Kuriger-Laber JK, Perry AE, Storm CA, Nelson HH: Skin Cancer Risk Is Modified by KIR/HLA Interactions That Influence the Activation of Natural Killer Immune Cells. Cancer research 2016, 76(2):370-376

108. Ewen EM, Pahl JHW, Miller M, Watzl C, Cerwenka A: KIR downregulation by IL-12/15/18 unleashes human NK cells from KIR/HLA-I inhibition and enhances killing of tumor cells. Eur J Immunol 2018, 48(2):355-365.

109. Mahaweni NM, Ehlers FAI, Bos GMJ, Wieten L: Tuning Natural Killer Cell Anti-multiple Myeloma Reactivity by Targeting Inhibitory Signaling via KIR and NKG2A. Front Immunol 2018, 9:2848.

110. Verheyden S, Bernier M, Demanet C: Identification of natural killer cell receptor phenotypes associated with leukemia. Leukemia 2004, 18(12):2002-2007.

111. Zhang Y, Wang B, Ye S, Liu S, Liu M, Shen C, Teng Y, Qi J: Killer cell immunoglobulin-like receptor gene polymorphisms in patients with leukemia: possible association with susceptibility to the disease. Leukemia research 2010, 34(1):55-58.

112. Middleton D, Diler AS, Meenagh A, Sleator C, Gourraud PA: Killer immunoglobulin-like receptors (KIR2DL2 and/or KIR2DS2) in presence of their ligand (HLA-C1 group) protect against chronic myeloid leukaemia. Tissue antigens 2009, 73(6):553-560.

113. Augusto DG: The Impact of KIR Polymorphism on the Risk of Developing Cancer: Not as Strong as Imagined? Frontiers in Genetics 2016, 7(121).

114. Niepieklo-Miniewska W, Majorczyk E, Matusiak L, Gendzekhadze K, Nowak I, Narbutt J, Lesiak A, Kuna P, Poninska J, Pietkiewicz-Sworowska A et al: Protective effect of the KIR2DS1 gene in atopic dermatitis. Gene 2013, 527(2):594-600.

115. Saito H, Hirayama A, Umemura T, Joshita S, Mukawa K, Suga T, Tanaka E, Ota M: Association between KIR-HLA combination and ulcerative colitis and Crohn's disease in a Japanese population. PloS one 2018, 13(4):e0195778-e0195778.

116. Liang H-L, Ma S-J, Tan H-Z: Association between killer cell immunoglobulin-like receptor (KIR) polymorphisms and systemic lupus erythematosus (SLE) in populations: A PRISMA-compliant meta-analysis. Medicine (Baltimore) 2017, 96(10):e6166-e6166.

117. Mohammad-Ebrahim H, Kamali-Sarvestani E, Mahmoudi M, Beigy M, Karami J, Ahmadzadeh N, Shahram F: Association of killer cell immunoglobulin-like receptor (KIR) genes and their HLA ligands with susceptibility to Behcet's disease. Scandinavian journal of rheumatology 2018, 47(2):155-163.

118. van Duin D, Avery RK, Hemachandra S, Yen-Lieberman B, Zhang A, Jain A, Butler RS, Barnard J, Schold JD, Fung $\mathrm{J}$ et al: KIR and HLA interactions are associated with control of primary CMV infection in solid organ transplant recipients. American journal of transplantation : official journal of the American Society of Transplantation and the American Society of Transplant Surgeons 2014, 14(1):156-162.

119. Littera R, Piredda G, Argiolas D, Lai S, Congeddu E, Ragatzu P, Melis M, Carta E, Michittu MB, Valentini D et al: KIR and their HLA Class I ligands: Two more pieces towards completing the puzzle of chronic rejection and graft loss in kidney transplantation. PloS one 2017, 12(7):e0180831. 
120. Rajalingam R: The Impact of HLA Class I-Specific Killer Cell Immunoglobulin-Like Receptors on AntibodyDependent Natural Killer Cell-Mediated Cytotoxicity and Organ Allograft Rejection. Front Immunol 2016, $7(585)$

121. Neuchel C, Fürst D, Niederwieser D, Bunjes D, Tsamadou C, Wulf G, Pfreundschuh M, Wagner E, Stuhler G, Einsele $\mathrm{H}$ et al: Impact of Donor Activating KIR Genes on HSCT Outcome in C1-Ligand Negative Myeloid Disease Patients Transplanted with Unrelated Donors-A Retrospective Study. PloS one 2017, 12(1):e0169512-e0169512.

122. Shaffer BC, Hsu KC: How important is NK alloreactivity and KIR in allogeneic transplantation? Best Pract Res Clin Haematol 2016, 29(4):351-358.

123. Hiby SE, Apps R, Sharkey AM, Farrell LE, Gardner L, Mulder A, Claas FH, Walker JJ, Redman CW, Morgan Let al: Maternal activating KIRs protect against human reproductive failure mediated by fetal HLA-C2. The Journal of clinical investigation 2010, 120(11):4102-4110.

124. Hiby SE, Walker JJ, O'Shaughnessy K M, Redman CW, Carrington M, Trowsdale J, Moffett A: Combinations of maternal KIR and fetal HLA-C genes influence the risk of preeclampsia and reproductive success. The Journal of experimental medicine 2004, 200(8):957-965.

125. Hiby SE, Regan L, Lo W, Farrell L, Carrington M, Moffett A: Association of maternal killer-cell immunoglobulinlike receptors and parental HLA-C genotypes with recurrent miscarriage. Human reproduction (Oxford, England) 2008, 23(4):972-976.

126. Chazara O, Xiong S, Moffett A: Maternal KIR and fetal HLA-C: a fine balance. Journal of leukocyte biology 2011, 90(4):703-716.

127. Xiong S, Sharkey AM, Kennedy PR, Gardner L, Farrell LE, Chazara O, Bauer J, Hiby SE, Colucci F, Moffett A: Maternal uterine NK cell-activating receptor KIR2DS1 enhances placentation. The Journal of clinical investigation 2013, 123(10):4264-4272.

128. Kennedy PR, Chazara O, Gardner L, Ivarsson MA, Farrell LE, Xiong S, Hiby SE, Colucci F, Sharkey AM, Moffett A: Activating KIR2DS4 Is Expressed by Uterine NK Cells and Contributes to Successful Pregnancy. The Journal of Immunology 2016, 197(11):4292.

129. Wang S, Zhao YR, Jiao YL, Wang LC, Li JF, Cui B, Xu CY, Shi YH, Chen ZJ: Increased activating killer immunoglobulin-like receptor genes and decreased specific HLA-C alleles in couples with recurrent spontaneous abortion. Biochemical and biophysical research communications 2007, 360(3):696-701.

130. Faridi RM, Agrawal S: Killer immunoglobulin-like receptors (KIRs) and HLA-C allorecognition patterns implicative of dominant activation of natural killer cells contribute to recurrent miscarriages. Human reproduction (Oxford, England) 2011, 26(2):491-497.

131. Witt CS, Goodridge J, Gerbase-Delima MG, Daher S, Christiansen FT: Maternal KIR repertoire is not associated with recurrent spontaneous abortion. Human reproduction (Oxford, England) 2004, 19(11):2653-2657.

132. Cooper MA, Elliott JM, Keyel PA, Yang L, Carrero JA, Yokoyama WM: Cytokine-induced memory-like natural killer cells. Proceedings of the National Academy of Sciences of the United States of America 2009, 106(6):19151919 .

133. Sungur CM, Murphy WJ: Utilization of mouse models to decipher natural killer cell biology and potential clinical applications. Hematology American Society of Hematology Education Program 2013, 2013:227-233.

134. Carlyle JR, Mesci A, Fine JH, Chen P, Belanger S, Tai LH, Makrigiannis AP: Evolution of the Ly49 and Nkrp1 recognition systems. Seminars in immunology 2008, 20(6):321-330.

135. Hanke T, Takizawa H, McMahon CW, Busch DH, Pamer EG, Miller JD, Altman JD, Liu Y, Cado D, Lemonnier FA et al: Direct assessment of MHC class I binding by seven Ly49 inhibitory NK cell receptors. Immunity 1999, 11(1):67-77.

136. Eagle RA, Trowsdale J: Promiscuity and the single receptor: NKG2D. Nature reviews Immunology 2007, 7(9):737-744.

137. Herndler-Brandstetter D, Shan L, Yao Y, Stecher C, Plajer V, Lietzenmayer M, Strowig T, de Zoete MR, Palm NW, Chen J et al: Humanized mouse model supports development, function, and tissue residency of human natural killer cells. Proceedings of the National Academy of Sciences of the United States of America 2017, 114(45):E9626-e9634.

138. Douam F, Ziegler CGK, Hrebikova G, Fant B, Leach R, Parsons L, Wang W, Gaska JM, Winer BY, Heller B et al: Selective expansion of myeloid and NK cells in humanized mice yields human-like vaccine responses. Nature Communications 2018, 9(1):5031. 
139. Dudley DM, Aliota MT, Mohr EL, Weiler AM, Lehrer-Brey G, Weisgrau KL, Mohns MS, Breitbach ME, Rasheed $\mathrm{MN}$, Newman $\mathrm{CM}$ et al: A rhesus macaque model of Asian-lineage Zika virus infection. Nature Communications 2016, 7(1):12204.

140. Dijkman K, Sombroek CC, Vervenne RAW, Hofman SO, Boot C, Remarque EJ, Kocken CHM, Ottenhoff THM, Kondova I, Khayum MA et al: Prevention of tuberculosis infection and disease by local BCG in repeatedly exposed rhesus macaques. Nature Medicine 2019, 25(2):255-262.

141. Hatziioannou T, Ambrose Z, Chung NPY, Piatak M, Jr., Yuan F, Trubey CM, Coalter V, Kiser R, Schneider D, Smedley J et al: A macaque model of HIV-1 infection. Proceedings of the National Academy of Sciences of the United States of America 2009, 106(11):4425-4429.

142. Bjornson-Hooper ZB, Fragiadakis GK, Spitzer MH, Madhireddy D, Mcllwain D, Nolan GP: A comprehensive atlas of immunological differences between humans, mice and non-human primates. bioRxiv 2019:574160.

143. Hong H, Rajakumar P, Billingsley J, Reeves RK, Johnson R: No monkey business: why studying NK cells in nonhuman primates pays off. Front Immunol 2013, 4(32).

144. Reeves RK, Gillis J, Wong FE, Yu Y, Connole M, Johnson RP: CD16- natural killer cells: enrichment in mucosal and secondary lymphoid tissues and altered function during chronic SIV infection. Blood 2010, 115(22):4439-4446.

145. Okoye AA, DeGottardi MQ, Fukazawa Y, Vaidya M, Abana CO, Konfe AL, Fachko DN, Duell DM, Li H, Lum R et al: Role of IL-15 Signaling in the Pathogenesis of Simian Immunodeficiency Virus Infection in Rhesus Macaques. The Journal of Immunology 2019:ji1900792.

146. Vargas-Inchaustegui DA, Helmold Hait S, Chung HK, Narola J, Hoang T, Robert-Guroff M: Phenotypic and Functional Characterization of Circulatory, Splenic, and Hepatic NK Cells in Simian Immunodeficiency VirusControlling Macaques. The Journal of Immunology 2017, 199(9):3202.

147. Adams EJ, Parham P: Species-specific evolution of MHC class I genes in the higher primates. Immunological reviews 2001, 183:41-64.

148. Valiante NM, Uhrberg M, Shilling HG, Lienert-Weidenbach K, Arnett KL, D’Andrea A, Phillips JH, Lanier LL, Parham P: Functionally and structurally distinct NK cell receptor repertoires in the peripheral blood of two human donors. Immunity 1997, 7(6):739-751.

149. Guethlein LA, Older Aguilar AM, Abi-Rached L, Parham P: Evolution of killer cell Ig-like receptor (KIR) genes: definition of an orangutan KIR haplotype reveals expansion of lineage III KIR associated with the emergence of MHC-C. Journal of immunology (Baltimore, Md : 1950) 2007, 179(1):491-504.

150. Moesta AK, Abi-Rached L, Norman PJ, Parham P: Chimpanzees use more varied receptors and ligands than humans for inhibitory killer cell Ig-like receptor recognition of the MHC-C1 and MHC-C2 epitopes. Journal of immunology (Baltimore, Md : 1950) 2009, 182(6):3628-3637.

151. Abi-Rached L, Moesta AK, Rajalingam R, Guethlein LA, Parham P: Human-specific evolution and adaptation led to major qualitative differences in the variable receptors of human and chimpanzee natural killer cells. PLoS genetics 2010, 6(11):e1001192.

152. Martin AM, Kulski JK, Gaudieri S, Witt CS, Freitas EM, Trowsdale J, Christiansen FT: Comparative genomic analysis, diversity and evolution of two KIR haplotypes A and B. Gene 2004, 335:121-131.

153. Leaton LA, Shortt J, Kichula KM, Tao S, Nemat-Gorgani N, Mentzer AJ, Oppenheimer SJ, Deng Z, Hollenbach JA, Gignoux CR et al: Conservation, Extensive Heterozygosity, and Convergence of Signaling Potential All Indicate a Critical Role for KIR3DL3 in Higher Primates. Front Immunol 2019, 10(24).

154. Hershberger KL, Shyam R, Miura A, Letvin NL: Diversity of the killer cell Ig-like receptors of rhesus monkeys. Journal of immunology (Baltimore, Md: 1950) 2001, 166(7):4380-4390.

155. Boyson JE, Shufflebotham C, Cadavid LF, Urvater JA, Knapp LA, Hughes AL, Watkins DI: The MHC class I genes of the rhesus monkey. Different evolutionary histories of $\mathrm{MHC}$ class I and II genes in primates. Journal of immunology (Baltimore, Md : 1950) 1996, 156(12):4656-4665.

156. Doxiadis GGM, de Groot N, Otting N, Blokhuis JH, Bontrop RE: Genomic plasticity of the MHC class I A region in rhesus macaques: extensive haplotype diversity at the population level as revealed by microsatellites. Immunogenetics 2011, 63(2):73-83.

157. Otting N, Heijmans CM, Noort RC, de Groot NG, Doxiadis GG, van Rood JJ, Watkins DI, Bontrop RE: Unparalleled complexity of the $\mathrm{MHC}$ class I region in rhesus macaques. Proceedings of the National Academy of Sciences of the United States of America 2005, 102(5):1626-1631. 
158. Maxwell LD, Wallace A, Middleton D, Curran MD: A common KIR2DS4 deletion variant in the human that predicts a soluble KIR molecule analogous to the KIR1D molecule observed in the rhesus monkey. Tissue antigens 2002, 60(3):254-258.

159. Sambrook JG, Bashirova A, Palmer S, Sims S, Trowsdale J, Abi-Rached L, Parham P, Carrington M, Beck S: Single haplotype analysis demonstrates rapid evolution of the killer immunoglobulin-like receptor (KIR) loci in primates. Genome research 2005, 15(1):25-35.

160. Rosner C, Kruse PH, Hermes M, Otto N, Walter L: Rhesus macaque inhibitory and activating KIR3D interact with Mamu-A-encoded ligands. Journal of immunology (Baltimore, Md : 1950) 2011, 186(4):2156-2163.

161. Schafer JL, Colantonio AD, Neidermyer WJ, Dudley DM, Connole M, O'Connor DH, Evans DT: KIR3DL01 recognition of Bw4 ligands in the rhesus macaque: maintenance of Bw4 specificity since the divergence of apes and Old World monkeys. Journal of immunology (Baltimore, Md : 1950) 2014, 192(4):1907-1917.

162. Colantonio AD, Bimber BN, Neidermyer WJ, Jr., Reeves RK, Alter G, Altfeld M, Johnson RP, Carrington M, O'Connor DH, Evans DT: KIR polymorphisms modulate peptide-dependent binding to an MHC class I ligand with a Bw6 motif. PLoS Pathog 2011, 7(3):e1001316.

163. Banerjee P, Ries M, Janaka SK, Grandea AG, Wiseman R, O’Connor DH, Golos TG, Evans DT: Diversification of Bw4 Specificity and Recognition of a Nonclassical MHC Class I Molecule Implicated in Maternal-Fetal Tolerance by Killer Cell Ig-like Receptors of the Rhesus Macaque. Journal of immunology (Baltimore, Md : 1950) 2018, 201(9):2776-2786

164. Bimber BN, Evans DT: The killer-cell immunoglobulin-like receptors of macaques. Immunological reviews 2015 , 267(1):246-258

165. de Groot NG, Blokhuis JH, Otting N, Doxiadis GG, Bontrop RE: Co-evolution of the MHC class I and KIR gene families in rhesus macaques: ancestry and plasticity. Immunological reviews 2015, 267(1):228-245.

166. Blokhuis JH, van der Wiel MK, Doxiadis GG, Bontrop RE: The mosaic of KIR haplotypes in rhesus macaques. Immunogenetics 2010, 62(5):295-306.

167. Boyson JE, Iwanaga KK, Golos TG, Watkins DI: Identification of a novel MHC class I gene, Mamu-AG, expressed in the placenta of a primate with an inactivated G locus. Journal of immunology (Baltimore, Md: 1950) 1997, 159(7):3311-3321.

168. Graves T, Eichler E.E. and Wilson R.K. (2019) Macaca mulatta isolate AG07107 chromosome 19, whole genome shotgun sequence. Published by NCBI. https://www.ncbi.nlm.nih.gov/nuccore/ CM014354. Accessed 21 November 2019

169. Blokhuis JH, van der Wiel MK, Doxiadis GG, Bontrop RE: Evidence for balancing selection acting on KIR2DL4 genotypes in rhesus macaques of Indian origin. Immunogenetics 2009, 61(7):503-512.

170. Blokhuis JH, van der Wiel MK, Doxiadis GG, Bontrop RE: The extreme plasticity of killer cell Ig-like receptor (KIR) haplotypes differentiates rhesus macaques from humans. Eur J Immunol 2011, 41(9):2719-2728.

171. Moreland AJ, Guethlein LA, Reeves RK, Broman KW, Johnson RP, Parham P, O'Connor DH, Bimber BN: Characterization of killer immunoglobulin-like receptor genetics and comprehensive genotyping by pyrosequencing in rhesus macaques. BMC genomics 2011, 12:295

172. Hellmann I, Lim S-Y, Gelman RS, Letvin NL: Association of activating KIR copy number variation of NK cells with containment of SIV replication in rhesus monkeys. PLoS Pathog 2011, 7(12):e1002436-e1002436.

173. Hershberger KL, Shyam R, Miura A, Letvin NL: Diversity of the Killer Cell Ig-Like Receptors of Rhesus Monkeys. The Journal of Immunology 2001, 166(7):4380.

174. Chaichompoo P, Bostik P, Stephenson S, Udompunturuk S, Kobkitjaroen J, Pattanapanyasat K, Ansari AA: Multiple KIR gene polymorphisms are associated with plasma viral loads in SIV-infected rhesus macaques. Cellular immunology 2010, 263(2):176-187.

175. Kruse PH, Rosner C, Walter L: Characterization of rhesus macaque KIR genotypes and haplotypes. Immunogenetics 2010, 62(5):281-293.

176. Bimber BN, Moreland AJ, Wiseman RW, Hughes AL, O'Connor DH: Complete characterization of killer Ig-like receptor (KIR) haplotypes in Mauritian cynomolgus macaques: novel insights into nonhuman primate KIR gene content and organization. Journal of immunology (Baltimore, Md : 1950) 2008, 181(9):6301-6308.

177. Prall TM, Graham ME, Karl JA, Wiseman RW, Ericsen AJ, Raveendran M, Alan Harris R, Muzny DM, Gibbs RA, Rogers J et al: Improved full-length killer cell immunoglobulin-like receptor transcript discovery in Mauritian cynomolgus macaques. Immunogenetics 2017, 69(5):325-339. 
178. Watson JD, Crick FHC: Molecular Structure of Nucleic Acids: A Structure for Deoxyribose Nucleic Acid. Nature 1953, 171(4356):737-738.

179. Gilbert W, Maxam A: The nucleotide sequence of the lac operator. Proceedings of the National Academy of Sciences of the United States of America 1973, 70(12):3581-3584.

180. Maxam AM, Gilbert W: A new method for sequencing DNA. Proceedings of the National Academy of Sciences of the United States of America 1977, 74(2):560-564.

181. Sanger F, Nicklen S, Coulson AR: DNA sequencing with chain-terminating inhibitors. Proceedings of the National Academy of Sciences of the United States of America 1977, 74(12):5463-5467.

182. Smith LM, Sanders JZ, Kaiser RJ, Hughes P, Dodd C, Connell CR, Heiner C, Kent SB, Hood LE: Fluorescence detection in automated DNA sequence analysis. Nature 1986, 321(6071):674-679.

183. Finishing the euchromatic sequence of the human genome. Nature 2004, 431(7011):931-945.

184. Eid J, Fehr A, Gray J, Luong K, Lyle J, Otto G, Peluso P, Rank D, Baybayan P, Bettman B et al: Real-time DNA sequencing from single polymerase molecules. Science (New York, NY) 2009, 323(5910):133-138.

185. Levene MJ, Korlach J, Turner SW, Foquet M, Craighead HG, Webb WW: Zero-mode waveguides for singlemolecule analysis at high concentrations. Science (New York, NY) 2003, 299(5607):682-686.

186. Church G, Deamer David W, Branton D, Baldarelli R, Kasianowicz J: Characterization Of Individual Polymer Molecules Based On Monomer-interface Interactions. In. EP: Hardvard College Univ California; 2008.

187. Laszlo AH, Derrington IM, Ross BC, Brinkerhoff H, Adey A, Nova IC, Craig JM, Langford KW, Samson JM, Daza R et al: Decoding long nanopore sequencing reads of natural DNA. Nature biotechnology 2014, 32(8):829-833.

188. Flusberg BA, Webster DR, Lee JH, Travers KJ, Olivares EC, Clark TA, Korlach J, Turner SW: Direct detection of DNA methylation during single-molecule, real-time sequencing. Nature methods 2010, 7(6):461-465.

189. Ni P, Huang N, Zhang Z, Wang D-P, Liang F, Miao Y, Xiao C-L, Luo F, Wang J: DeepSignal: detecting DNA methylation state from Nanopore sequencing reads using deep-learning. Bioinformatics 2019.

190. Kraft F, Kurth I: Long-read sequencing in human genetics. medizinische genetik 2019, 31(2):198-204.

191. Joag SV, Stephens EB, Adams RJ, Foresman L, Narayan O: Pathogenesis of SIVmac Infection in Chinese and Indian Rhesus Macaques: Effects of Splenectomy on Virus Burden. Virology 1994, 200(2):436-446.

192. Zhou Y, Bao R, Haigwood NL, Persidsky Y, Ho W-z: SIV infection of rhesus macaques of Chinese origin: a suitable model for HIV infection in humans. Retrovirology 2013, 10(1):89. 



\title{
Human and rhesus
}

\section{macaque KIR \\ haplotypes defined by their transcriptomes}

\author{
Jesse Bruijnesteijn ${ }^{1}$, Marit K. H. van der Wiel' \\ Wendy T. N. Swelsen ${ }^{2}$, Nel Otting ${ }^{1}$, Annemiek J. M. \\ de Vos-Rouweler ${ }^{1}$, Diënne Elferink ${ }^{3}$, Gaby G. Doxiadis ${ }^{1}$, \\ Frans H. J. Claas ${ }^{3}$, Neubury M. Lardy ${ }^{2}$, Natasja G. de Groot ${ }^{1}$ \\ and Ronald E. Bontrop ${ }^{1,4, *}$
}

${ }^{1}$ Comparative Genetics and Refinement,

Biomedical Primate Research Centre, 2288 GJ Rijswijk, The Netherlands 2Sanquin, Dept. of Immunogenetics, 1066 CX Amsterdam, The Netherlands

${ }^{3}$ LUMC, Immunohematology and Blood Transfusion, 2300 RC Leiden,

The Netherlands

${ }^{4}$ Theoretical Biology and Bioinformatics, Utrecht University, 3584 CH Utrecht, The Netherlands

*Corresponding author:

Prof. Dr. Ronald E. Bontrop

bontrop@bprc.nl

Tel. nr. +31-15-2842699

Fax nr. +31-15-2842600 


\begin{abstract}
The killer-cell immunoglobulin-like receptors (KIR) play a central role in the immune recognition in infection, pregnancy and transplantation through their interactions with MHC class I molecules. KIR genes display abundant copy number variation as well as high levels of polymorphism. As a result, it is challenging to characterize this structurally dynamic region. KIR haplotypes have been analysed in different species using conventional characterization methods, such as Sanger sequencing and Roche/454 pyrosequencing. However, these methods are time-consuming and often failed to define complete haplotypes, or do not reach allele-level resolution. In addition, most analyses were performed on genomic DNA, and thus were lacking substantial information about transcription and its corresponding modifications. In this communication, we present a Single-Molecule, Real-Time (SMRT) sequencing approach, using Pacific Bioscience's (PacBio) Sequel platform to characterize the KIR transcriptomes in human and rhesus macaque (Macaca mulatta) families. This highresolution approach allowed the identification of novel Mamu-KIR alleles, the extension of reported allele sequences, and the determination of human and macaque KIR haplotypes. In addition, multiple recombinant KIR genes were discovered, all located on contracted haplotypes, which were likely the result of chromosomal rearrangements. The relatively high number of contracted haplotypes discovered might be indicative of selection on small KIR repertoires and/or novel fusion gene products. This nextgeneration method provides an improved high-resolution characterization of the KIR cluster in humans and macaques, which eventually may aid in a better understanding and interpretation of KIR allele associated diseases, as well as the immune response in transplantation and reproduction.
\end{abstract}

\title{
Introduction
}

Killer-cell Immunoglobulin-like Receptors (KIR) are expressed on natural killer (NK) cells and subsets of $\mathrm{T}$ cells $(1,2)$, and play a key role in immune recognition through interactions with the highly polymorphic major histocompatibility complex (MHC) class I molecules $(3,4)$. For example, KIR may play an important role in the detection of aberrant $\mathrm{MHC}$ class I expression on tumor and virally infected cells, and their subsequent elimination $(5,6)$. KIR are type I transmembrane glycoproteins that consist of two or three extracellular Ig-like domains (2D or 3D) as well as a stem, transmembrane region and cytoplasmic tail. The length of the cytoplasmic tail can be either long ( $\mathrm{L})$, including two ITIM motifs, or short (S), and characterizes inhibitory or activating KIR, respectively. KIR with one extracellular Ig-like domain and a truncated cytoplasmic tail (KIR1D) are observed in some non-human primate species, and seem to have no counterpart in humans $(7,8)$. The nomenclature of the $K I R$ genes is based on the functional (L, S) and structural characteristics (2D or 3D), and takes into account allelic variation as well (9). 
In humans, the KIR gene cluster is located within the leukocyte receptor complex (LRC) on chromosome 19q13.4, and displays copy number variation (CNV) at the population level, as reflected by a variable number of tandemly arranged KIR genes $(10,11)$. A KIR haplotype contains seven to twelve expressed genes, three of which are considered framework genes: KIR3DL3, KIR3DL2, and KIR2DL4 (12). A fourth framework gene is KIR3DP1, which is a pseudo-gene. Based on the genetic make up, the human $K I R$ haplotypes can be categorized into two groups (13). Group A haplotypes are characterized by seven KIR genes, including the framework genes and only the activating KIR2DS4 structure, whereas group B haplotypes can contain up to twelve genes including the framework genes and multiple activating receptors. The KIR genes can be further divided into phylogenetic lineages (I, II, III and V), each characterized by structure and MHC class I specificity; lineage I includes KIR2DL4 and KIR2DL5, lineage II includes KIR3DL1/S1 and KIR3DL2, lineage III includes KIR2DS1/2, KIR2DL1/2/3, KIR2DS3/5, KIR2DS4 and the pseudo-genes, and lineage $V$ includes KIR3DL3.

In addition to the CNV, allelic polymorphism is another important feature of the KIR gene system. In humans, the greatest expansion is observed for $K I R$ lineage III genes in both the telomeric and centromeric region of the haplotype. A total of fifteen human $K I R$ genes and two pseudo-genes have been characterized, and up to 907 unique fulllength KIR alleles have been catalogued (14).

As various KIR may bind specific but differential structures on MHC class I molecules, the KIR repertoire influences, in part, the variability of the immune response. Since both the MHC and KIR gene systems display substantial levels of polymorphism and segregate as independent entities located on different chromosomes, the potential repertoire of MHC-KIR interactions may vary considerably, even among related individuals within a family. The presence or absence of certain KIR alleles and MHC-KIR interactions are associated with disease susceptibility and its progress, but may also play a role in transplantation and reproductive biology $(5,6,15,16)$.

In recent years, our understanding of the biology and evolution of the KIR gene system has greatly expanded, although some key questions remain to be answered. Suitable animal models to study KIR-related diseases are more or less confined to nonhuman primate species, since rodent species have another system executing similar tasks that arose as result of convergent evolution $(17,18)$. Macaques, for example, share a close evolutionary relationship with humans, which is evidenced by similar pathology and immune responses in models for infectious and autoimmune diseases (19-21). Initial genomic characterization of the $K I R$ gene repertoire in rhesus macaques (Macaca mulatta, Mamu) highlighted substantial similarities along with some differences as compared to humans $(8,22-24)$. For example, the macaque KIR gene cluster shows an extreme expansion of lineage II KIR genes, mainly in the telomeric part of the haplotype, which might be associated with the multiplicity of the KIRinteracting $M H C-A$ and $-B$ genes $(25,26)$. This extensive gene copy number variation exceeds the lineage III KIR expansion observed in humans. Thus far, twenty-two KIR genes and 218 alleles have been reported in macaques (7, 22, 27-35). KIR haplotypes in macaques can contain up to eleven genes, some including Mamu-KIR2DLO4 and 
Mamu-KIR1D, which belong to KIR lineages I and III, respectively. The human KIR haplotypic division differentiating between the more activating (B) and the more inhibitory (A) haplotypes is not as obvious for macaques $(22,26)$.

A limited number of macaque haplotypes has been characterized by studying segregation in families in combination with conventional sequencing methods, leading to haplotype definitions that were based on the presence of both partial and fulllength cDNA sequences. Although these methods provided insights, they were either not always sufficient to resolve allele-level haplotypes, or to identify genes with low transcription levels. In contrast, most human KIR haplotypes were characterized by determining the presence or absence of KIR genes at the genomic DNA (gDNA) level. As a consequence, crucial information on the allele level, copy number variation, transcription level, and transcriptional modifications, such as splicing, may be missed. As particular KIR alleles are expected to be associated with health and disease, a comprehensive method is required to characterize the complete KIR transcriptome. Here we report a next-generation Single-Molecule, Real-Time (SMRT) sequencing method on the PacBio Sequel platform, which allowed us to obtain full-length KIR transcriptomes, as well as KIR haplotypes for both human and rhesus macaque families. This approach provides a significant step forward, which may aid in a better understanding and interpretation of KIR-allele associated diseases, as well as the immune reactivity in transplantation and reproductive biology.

\section{Materials \& method}

\section{Animals and cells}

A large pedigree-based Indian rhesus macaque family, with a total of thirty animals, was selected from the self-sustaining colony housed at the Biomedical Primate Research Centre (BPRC) (Figure 1). EDTA or heparin whole blood samples were obtained during regular annual health checks, and peripheral blood mononuclear cells (PBMC) were isolated from heparin blood samples. PBMCs of fifteen related humans were provided by the immunohematology and blood transfusion department of the Leiden University Medical Center (Figure 4). Informed consent was obtained from all participants.

\section{RNA / gDNA extraction and cDNA synthesis}

Total RNA was extracted directly from EDTA whole blood samples or from $\pm 15 \times 106$ PBMCs with RNeasy Mini Kit (Qiagen, Valencia, CA, USA) in accordance with the manufacturer's instructions. First-strand cDNA was synthesized with the RevertAid First Strand cDNA Synthesis Kit (Invitrogen, Carlsbad, CA, USA) using oligo(dT) ${ }_{18}$ primers. Genomic DNA was extracted from EDTA whole blood samples by a standard salting-out procedure, or from $\pm 15 \times 10^{6}$ PBMCs with an AllPrep RNA/DNA easy Mini Kit (Qiagen, Valencia, CA, USA) according to the manufactures instructions. 


\section{PacBio Sequel PCR amplification, sample purification and SMRTbell libraries}

Polymerase chain reaction (PCR) with different primer sets (Table I) was performed on cDNA using Phusion Hot Start II DNA Polymerase (Thermo Fisher Scientific, Waltham, MA, USA) to obtain full-length macaque and human KIR amplicons. Each primer was tagged at the 5' end with a 16 bp barcode, designed for the PacBio platform, to identify samples by unique barcode combinations. Thermal cycling conditions were: denaturation at $98{ }^{\circ} \mathrm{C}$ for $2 \mathrm{~min}$, followed by 32 cycles of $98{ }^{\circ} \mathrm{C}$ for $20 \mathrm{~s}, 66^{\circ} \mathrm{C}$ for $45 \mathrm{~s}$, and $72{ }^{\circ} \mathrm{C}$ for $2 \mathrm{~min}$, except for the primer set derived from Moreland and colleagues (24): denaturation at $98^{\circ} \mathrm{C}$ for $2 \mathrm{~min} ; 5$ cycles of $98^{\circ} \mathrm{C}$ for $20 \mathrm{~s}, 68^{\circ} \mathrm{C}$ for $5 \mathrm{~s}, 66^{\circ} \mathrm{C}$ for $5 \mathrm{~s}, 63{ }^{\circ} \mathrm{C}$ for $30 \mathrm{~s}, 60{ }^{\circ} \mathrm{C}$ for $5 \mathrm{~s}$, and $72{ }^{\circ} \mathrm{C}$ for $2 \mathrm{~min} ; 29$ cycles of $98^{\circ} \mathrm{C}$ for $20 \mathrm{~s}, 63^{\circ} \mathrm{C}$ for $30 \mathrm{~s}$, and $72{ }^{\circ} \mathrm{C}$ for $2 \mathrm{~min}$. Appropriately sized PCR products of approximately 1250 bp were selected by gel electrophoresis and purified using a GeneJet Gel extraction kit (Invitrogen, Carlsbad, CA, USA). The amplified KIR amplicons were pooled and purified twice, using AMPure XP beads (Beckman-Coulter, Woerden, The Netherlands) at a 1:1 bead to DNA volume ratio. The DNA concentration of purified pooled samples ( $>1 \mu \mathrm{g}$ total DNA) was measured using the Qubit dsDNA HS assay kit and Qubit 2.0 Fluorometer (Thermo Fisher Scientific, Waltham, MA, USA).

SMRTbell libraries were generated according to the PacBio Amplicon Template Preparation protocol for circular consensus sequences (CCS) (36). In brief, PCR product pools were end-repaired, and hairpin adapters were subsequently incorporated using the PacBio DNA Template Prep Kit 2.0. After the removal of failed ligation products, the SMRTbell templates were purified with $0.6 x$ volume of AMPure XP beads. The volume of sequencing primer and polymerase was determined using the PacBio calculator. Polymerase-bound SMRTbells were MagBead loaded over zero-mode waveguides and immobilized. SMRTbell library generation and sequencing were performed by the Leiden Genome Technology Centre (LGTC) using a PacBio Sequel instrument with P6C4 sequencing chemistry.

\section{PacBio Sequel data analysis}

Circular consensus sequences were obtained and selected for high read quality (rqvalue of 0.99 or higher). The data was demultiplexed based on unique barcoding, and were used to type individual samples.

Geneious Pro R10 software (37) was used to map the reads to a reference database, including all reported full-length and partial human or macaque KIR CDNA sequences $(7,14,22,27-35)$, to identify $100 \%$ matched reads (100\% overlap, $0 \%$ mismatch, maximum ambiguity $=1$ ). The remaining reads were De Novo assembled, and the consensus of each contig was phylogenetically aligned with the human or macaque KIR sequence reference database. Official designations of novel sequences were determined by comparison with the reference sequence databases and by phylogenetic analysis, and were provided by the Immuno Polymorphism Database (IPD), which will shortly release a database with non-human primate KIR sequences. The novel sequences were confirmed when observed in two independent PCRs or shown to 
segregate into families, and were subsequently submitted to the European Nucleotide Archive (ENA) and assigned an association number (https://www.ebi.ac.uk/ena/data/ view/PRJEB22235).

\section{Allele confirmation by Sanger sequencing}

Alleles that could not be confirmed by segregation and had low PacBio read counts $(<3$ reads), or alleles that seemed to be duplicated on a single haplotype, were confirmed by Sanger sequencing. To amplify products that distinguished the different genes or alleles, specific primers were designed within the exons (Supp. Table I). For all primers, the PCR conditions were: denaturation at $98{ }^{\circ} \mathrm{C}$ for $2 \mathrm{~min}$, followed by 32 cycles of $98^{\circ} \mathrm{C}$ for $20 \mathrm{~s}, 66^{\circ} \mathrm{C}$ for $45 \mathrm{~s}$, and $72^{\circ} \mathrm{C}$ for $2 \mathrm{~min}$. PCR products were subjected to gel electrophoresis, and bands of the appropriate size were purified with a GeneJet Gel extraction kit. For Mamu-KIR3DLO7, Mamu-KIR3DLO5 and Mamu-KIR3DSO2 cloning was performed as previously described (28). Sequencing of the PCR products or isolated cloned amplicons was performed on a 3500XL Genetic Analyzer automatic sequencer (Applied Biosystems, Foster City, USA). Sequences were analyzed with SeqMan Pro (Dnastar, Inc., Madison, USA) and MacVector (MacVector, Inc., Cambridge, UK) software.

\section{Genomic DNA KIR typing}

In addition to typing at the transcription level, both human and macaque samples were also typed at the gDNA level for the presence or absence of KIR genes. Rhesus macaque DNA samples were typed using quantitative PCR (qPCR) and melt curve analysis as previously described (23). Human KIR genotyping was performed by Sanquin (Dept. of Immunogenetics, Netherlands) using the Olerup SSP ${ }^{\circledR}$ KIR typing kit (Olerup SSP AB, Stockholm, Sweden) in accordance with the manufacturer's instructions.

\section{Results}

\section{Comparison of the conventional characterization strategies versus the Pacbio Sequel platform}

The rhesus macaque KIR system was previously characterized by cloning and Sanger sequencing, Roche/454 pyrosequencing, and microsatellite analysis, which resulted in a database of 218 partial and full-length Mamu-KIR alleles $(22-24,27,33,38)$. Validation of these sequences was provided by independent amplifications and, whenever possible, segregation studies. However, these characterization strategies were timeconsuming, and were often insufficient to resolve full-length allele sequences.

Experience taught that the Pacbio Sequel platform offers a substantial number of improvements compared to conventional sequencing strategies, such as higher throughput and longer reads with high accuracy by circular consensus sequencing (CCS) $(39,40)$. Taking these advantages into account, we set up a pipeline to characterize the KIR gene system using a PacBio Sequel platform. Initially, we calibrated the PacBio 
platform by re-analyzing macaque blood samples that had been previously typed for $K I R$ by a conventional methodology. With the current PacBio sequencing protocol, we confirmed in considerably less time the rhesus macaque KIR results that had been obtained earlier. Furthermore, additional KIR alleles and genes were identified, and partial sequences that had been missed by conventional methods could be extended. In the following set-ups, we used a family segregation concept, so that identical sequences could be obtained and confirmed by analyzing different but related individuals.

\section{Full-length KIR allele discovery at the transcription level in rhesus macaques}

The previously published macaque KIR3D/1D and KIR2DLO4 primers located in conserved regions of the $5^{\prime}$ and $3^{\prime}$ UTR were barcoded with PacBio sequence tags, and used to amplify full-length KIR cDNA transcripts $(22,24)$. A combination of two generic Mamu-KIR3D/1D and one specific Mamu-KIR2DLO4 primer sets was required to amplify the complete KIR transcriptome in macaques (Table I).

A family of thirty macaques was selected that originated from eight founders and covered four generations, which allowed an extensive segregation study (Figure 1). An average of approximately 9,000 Mamu-KIR3D/1D and 5,800 Mamu-KIR2DL04 reads were obtained per animal ( $\pm 100,000$ reads/PacBio Sequel cell), of which $20-45 \%$ were perfectly matched with the Mamu-KIR allele library that consisted of 218 annotated Mamu-KIR3D/1D and Mamu-KIR2DLO4 sequences. The remaining reads that did not match with the Mamu-KIR library were novel alleles, partial sequences or sequences containing random single nucleotide gaps, which had been introduced by PacBio sequencing. A total of twenty-nine unreported Mamu-KIR alleles were identified in thirty related rhesus macaques (Tables II and III). Six new KIR alleles were identified for

Table I. Overview of forward (Fw) and reverse (Rv) primers used for PacBio PCR amplification of full lenght KIR in humans and macaques. To amplify different KIR genes, wobbles had to be introduced in primer sequences: cytosine or thymine $(Y)$, adenine or guanine $(R)$, guanine or thymine $(K)$ and adenine or thymine (W). The human KIR2D/3D primers were designed for this study to amplify all KIR genes, except for KIR2DL4, KIR2DL5, and KIR3DL3. Human KIR2DL4 was specifically amplified by a seperate primer set.

\begin{tabular}{|c|c|c|c|}
\hline Target & Fw / Rv & Primer sequence 5' - 3' & Source \\
\hline \multirow{2}{*}{ Mamu-KIR3D/1D } & $\mathrm{FW}$ & CAGCACCATGTCGCTCAT & \multirow{2}{*}{ Moreland et al. [24] } \\
\hline & Rv & GGGGTCAAGTGAAGTGGAGA & \\
\hline \multirow{2}{*}{ Mamu-KIR3D/1D } & Fw & AACATCCTGTGYRCTGCKGAGCWGAG & \multirow{2}{*}{ Blokhuis et al. [22] } \\
\hline & Rv & TGGAKAATTGTGGGYTAAGCAARGGAG & \\
\hline \multirow{2}{*}{ Mamu-KIR2DL4 } & Fw & CСАСАTССTCTGCACCGGTCAGTC & \multirow{2}{*}{ Blokhuis et al. [22] } \\
\hline & $\mathrm{Rv}$ & GCAGGGGTCAAGTGAAGGGGAGAA & \\
\hline \multirow{2}{*}{ Human $K I R 2 D / 3 D$} & Fw & CGTCAYCCTCCCATGATGTGG & \multirow{2}{*}{ Designed for this study } \\
\hline & Rv & GTTGGAGAGGTGGGCAGG & \\
\hline \multirow{2}{*}{ Human KIR2DL4 } & Fw & ССТСАССАСАТССТСТGCAC & \multirow{2}{*}{ Designed for this study } \\
\hline & Rv & GGTGTGAGGAAGAGTGATGCT & \\
\hline
\end{tabular}




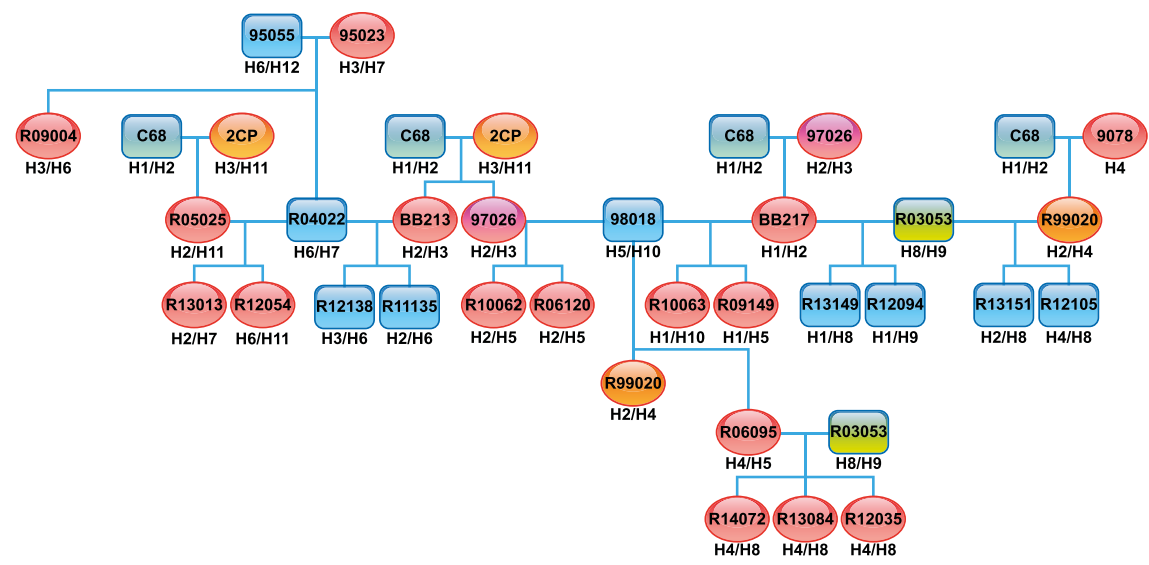

Figure 1. Pedigree of an Indian rhesus macaque family comprising thirty members. Depicted are the sires (blue squares) and dames (red ovals) with their offspring. Five animals (C68, 2CP, 97026, R99020, and R03053) are present in the pedigree multiple times, and are depicted by a different color. Different haplotypes are indicated by $\mathrm{H} 1, \mathrm{H} 2$, and so on. All haplotypes are found in at least two animals, except for $\mathrm{H} 12$, which did not segregate from sire 95055 into any of the offspring. Although no blood samples were obtained from dame $9078, \mathrm{H} 4$ could be deduced based on the offspring's genetic content.

Mamu-KIR3DLO7 and Mamu-KIR3DL20, whereas for the other KIR genes, two, one, or no new alleles were detected.

In two of the newly detected alleles, premature stop codons were identified, but both were located in exon 9. For example, an insertion of a single cytosine (C) was found at base pair position 1014 in Mamu-KIR3DL11*009 (LT906600), which resulted in a frame shift and the introduction of an early stop codon at base pair position 1171 (exon 9). This cytosine insertion was confirmed using multiple independent PCRs, and the frame shift might suggest a Mamu-KIR3DL11 isoform with a truncated cytoplasmic tail, thereby lacking the ability to signal via ITIMs. In Mamu-KIR3DL05*013 (LT906588), a point mutation at base pair position 1294 (exon 9) introduced a stop codon, which resulted in a transcript likely to encode a KIR3DL protein with only one ITIM.

In addition to novel alleles, six previously reported Mamu-KIR sequences were extended, and another thirty alleles were confirmed. Overall, of the sixty-nine KIR genes/alleles identified in the rhesus macaque family (Table II), almost half of them were novel, illustrating the power of the platform and the extensive allelic polymorphism of the macaque KIR gene system.

\section{Recombinant Mamu-KIR genes}

In addition to allelic polymorphism and CNV, the plasticity of the KIR gene system is also reflected by recombination events, such as inter- and intrachromosomal rearrangements, which might result in the formation of recombinant in-frame KIR genes (Figure 2A, B) (41-44). This type of generation of novel gene entities, caused by the fusion of different genes, has been described in humans, but thus far has not been 
Table II. Reported and novel alleles per Mamu-KIR gene. In total, 218 Mamu-KIR alleles are reported and used as reference database in this communication. Four recombinant and twenty-nine novel alleles were discovered and named by phylogenetic analysis. The recombinant genes consist of two segments from different KIR genes. Thirty-six alleles were confirmed or extended. In total, 69 Mamu-KIR alleles were reported in the studies rhesus macaque family.

\begin{tabular}{lccclccclcll}
\hline Gene & $\mathbf{R}$ & $\mathbf{C}$ & $\mathbf{N}$ & Gene & $\mathbf{R}$ & $\mathbf{C}$ & $\mathbf{N}$ & Gene & $\mathbf{R}$ & $\mathbf{C}$ & $\mathbf{N}$ \\
\hline $1 D$ & 3 & 1 & 0 & $3 D L 07$ & 13 & 1 & 6 & $3 D S 04$ & 9 & 0 & 0 \\
\hline $2 D L 4$ & 28 & 5 & 0 & $3 D L 08$ & 12 & 4 & 1 & $3 D S 05$ & 5 & 2 & 1 \\
\hline $3 D L 01$ & 28 & 7 & 2 & $3 D L 10$ & 8 & 1 & 2 & $3 D S 06$ & 8 & 1 & 1 \\
\hline $3 D L 02$ & 9 & 1 & 0 & $3 D L 11$ & 7 & 0 & 1 & $3 D S W 07$ & 3 & 0 & 0 \\
\hline $3 D L W 03$ & 5 & 0 & 2 & $3 D L 20$ & 15 & 4 & 6 & $3 D S W 08$ & 12 & 2 & 1 \\
\hline $3 D L 04$ & 4 & 0 & 0 & $3 D S 01$ & 5 & 1 & 1 & $3 D S w 09$ & 6 & 1 & 1 \\
\hline $3 D L 05$ & 14 & 2 & 2 & $3 D S 02$ & 17 & 2 & 2 & Recombinant & 0 & 0 & 4 \\
\hline $3 D L 06$ & 2 & 0 & 0 & $3 D S 03$ & 5 & 1 & 0 & Total & 218 & 36 & 33 \\
\hline
\end{tabular}

$\mathbf{R}=$ Reported alleles, $\mathbf{C}=$ Confirmed genes, $\mathbf{N}=$ Novel alleles

Table III. Overview of twenty-nine novel, four recombinant, and six extended $K I R$ genes observed in thirty related rhesus macaques. Official designations of novel sequences were determined by comparison with the reference sequence database, containing 218 reported Mamu-KIR sequences, and were provided by the Immuno Polymorphism Database (IPD), which will shortly release a database with non-human primate KIR sequences.The number of animals that contained the allele and the European Nucleotide Archive (ENA) accession numbers are listed (https://www.ebi.ac.uk/ena/). E, extended KIR sequence; R, recombinant KIR sequence.

\begin{tabular}{lcc}
\hline Gene name & $\begin{array}{c}\text { Number } \\
\text { of } \\
\text { animals }\end{array}$ & $\begin{array}{c}\text { ENA } \\
\text { accession } \\
\text { number }\end{array}$ \\
\hline Mamu-KIR3DL01*002:02 & 11 & LT906583 \\
\hline Mamu-KIR3DL01*028 & 3 & LT906584 \\
\hline Mamu-KIR3DLw03*006:01 & 6 & LT906585 \\
\hline Mamu-KIR3DLw03*007 & 2 & LT906586 \\
\hline Mamu-KIR3DL05*012 & 6 & LT906587 \\
\hline Mamu-KIR3DL05*013 & 2 & LT906588 \\
\hline Mamu-KIR3DL07*014 & 11 & LT906589 \\
\hline Mamu-KIR3DL07*016 & 5 & LT906591 \\
\hline Mamu-KIR3DL07*017 & 6 & LT906592 \\
\hline Mamu-KIR3DL07*018 & 2 & LT906593 \\
\hline Mamu-KIR3DL07*019 & 2 & LT906594 \\
\hline Mamu-KIR3DL07*020 & 2 & LT906595 \\
\hline Mamu-KIR3DL08*012 & 6 & LT906596 \\
\hline Mamu-KIR3DL10*002:03 & 6 & LT906597 \\
\hline Mamu-KIR3DL10*007 & 11 & LT906598 \\
\hline Mamu-KIR3DL11*009 & 2 & LT906600 \\
\hline Mamu-KIR3DL20*016 & 6 & LT906601 \\
\hline Mamu-KIR3DL20*017 & 3 & LT906602 \\
\hline Mamu-KIR3DL20*018 & 17 & LT906603 \\
\hline Mamu-KIR3DL20*019 & 6 & LT906604 \\
\hline
\end{tabular}

\begin{tabular}{lcc}
\hline Gene name & $\begin{array}{c}\text { Number } \\
\text { of } \\
\text { animals }\end{array}$ & $\begin{array}{c}\text { ENA } \\
\text { accession } \\
\text { number }\end{array}$ \\
\hline Mamu-KIR3DL20*020 & 2 & LT906605 \\
\hline Mamu-KIR3DL20*022 & 3 & LT906607 \\
\hline Mamu-KIR3DSO1*004 & 2 & LT906608 \\
\hline Mamu-KIR3DS02*014 & 2 & LT906609 \\
\hline Mamu-KIR3DSO2*015:01 & 6 & LT906610 \\
\hline Mamu-KIR3DS05*004 & 3 & LT906611 \\
\hline Mamu-KIR3DSO6*007 & 7 & LT906612 \\
\hline Mamu-KIR3DSW08*010:02 & 2 & LT907836 \\
\hline Mamu-KIR3DSW09*007 & 3 & LT907837 \\
\hline Mamu-KIR3DLO2/3DL08A (R) & 7 & LT907838 \\
\hline Mamu-KIR3DLO2/3DL08B (R) & 2 & LT907839 \\
\hline Mamu-KIR3DL10A/3DL02 (R) & 7 & LT907840 \\
\hline Mamu-KIR3DL10B/3DL02 (R) & 1 & LT907841 \\
\hline Mamu-KIR3DLO1*002 (E) & 7 & LT963634 \\
\hline Mamu-KIR3DLO2*004:01 (E) & 5 & LT963635 \\
\hline Mamu-KIR3DLO5*006:01 (E) & 6 & LT963636 \\
\hline Mamu-KIR3DLO7*009:01 (E) & 3 & LT963637 \\
\hline Mamu-KIR3DL20*001 (E) & 6 & LT963638 \\
\hline Mamu-KIR3DS05*002:02 (E) & 12 & LT963639 \\
\hline
\end{tabular}


A

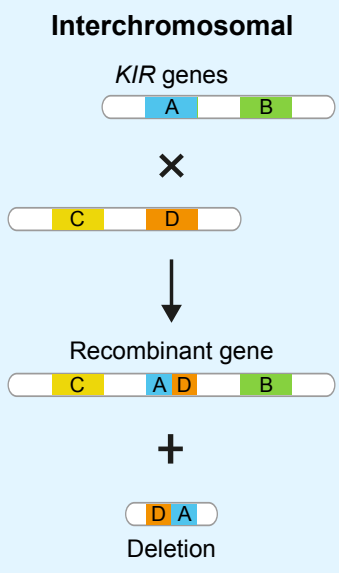

B

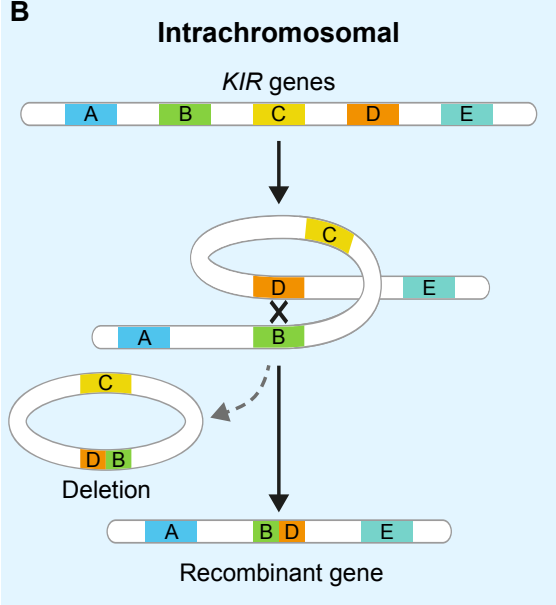

C

DO

D1

D2

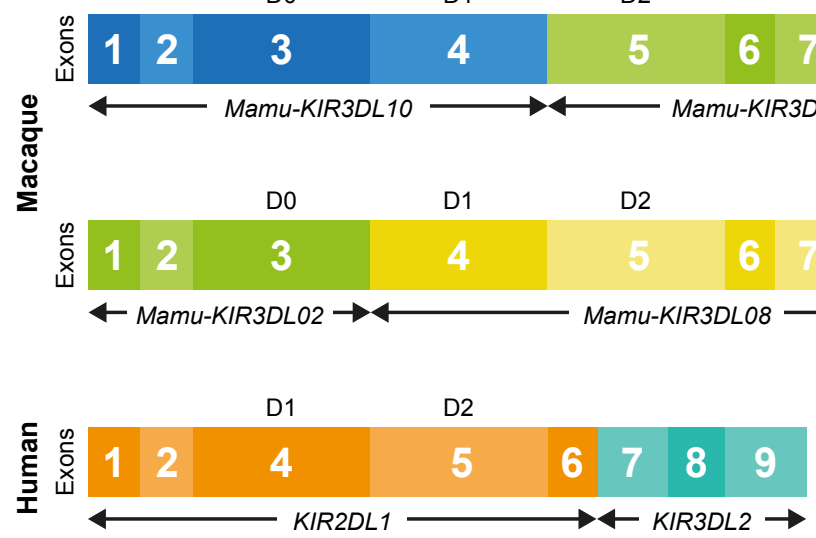

\section{D}

Figure 2. Chromosomal rearrangement mechanisms, inter- or intrachromosomal, which can result in recombinant KIR genes and the contraction of haplotypes. (A) Interchromosomal: recombination between two different (homologous) chromosomes can result in the generation of a novel recombinant gene and the deletion of gene segments. (B) Intrachromosomal: two genes on the same chromosome might align with each other, and subsequent rearrangements can result in the generation of a recombinant fusion gene and the deletion of a non-viable ring chromosome. The deletions described by (A) and (B), together with the generation of a recombinant gene, might explain the KIR haplotype contractions. (C) Two variants of recombinant gene transcripts observed in rhesus macaques are depicted. The $5^{\prime}$ end to exon 4 of $\mathrm{Mamu-}$ KIR3DL10 and exon 5 to the $3^{\prime}$ end of MamU-KIR3DLO2 encode one type of recombinant transcripts, with SNPS in the Mamu-KIR3DL10 segment. The other recombinant genes found in this study are encoded by the $5^{\prime}$ end to exon 3 of Mamu-KIR3DLO2 and exon 4 to the $3^{\prime}$ end of Mamu-KIR3DLO8, with variation in the MamuKIR3DL08 segment. Predicted KIR protein structures are schematically illustrated adjacent to the respective gene, in which the colors of the exons and protein segments resemble each other. (D) Human recombinant KIR transcript with the $5^{\prime}$ end to exon 6 of KIR2DL1 and exon 7 to the $3^{\prime}$ end of KIR3DL2. This transcript is likely to encode a KIR2D protein with the extracellular domains of KIR2DL1 and an intercellular tail of KIR3DL2, as schematically illustrated adjacent to the transcript. 
observed in rhesus macaques. In this study, comprising only one extended macaque family, four novel recombinant Mamu-KIR sequences were identified at the transcription level, composed of segments from two different $K I R$ genes (Figure $\mathbf{2 C}$ ). Two recombinant genes consisted of the $5^{\prime}$ end to exon 4 of Mamu-KIR3DL10 and exon 5 up to the $3^{\prime}$ end of Mamu-KIR3DLO2, but seemed to originate from two independent fusion events. In these two recombinant genes, most point mutations were observed in the Mamu-KIR3DL10 segment, whereas the Mamu-KIR3DLO2 part was less variable; we therefore refer to these recombinant genes as Mamu-KIR3DL1OA/3DLO2 and Mamu-KIR3DL1OB/3DLO2. The other two recombinant genes consisted of the $5^{\prime}$ end up to exon 3 of Mamu-KIR3DLO2 and exon 4 up to the $3^{\prime}$ end of Mamu-KIR3DLO8. These recombinant sequences can be distinguished by a synonymous and a nonsynonymous single nucleotide polymorphism (SNP) in the Mamu-KIR3DLO8 segment, and will be referred to as MamU-KIR3DLO2/3DLO8A and MamU-KIR3DLO2/3DLO8B.

The transcripts of the above-mentioned recombinant genes encoded three extracellular domains and a long cytoplasmic tail, suggesting an inhibitory function (Figure 2C). The recombinant genes were confirmed by independent PCRs and/or segregation into families. All four recombinant genes contained a segment of MamuKIR3DLO2, suggesting that this gene is highly susceptible to engaging in fusion events. However, recombinant genes containing segments from other inhibitory, activating, or pseudo-genes might be discovered when larger populations are studied.

\section{Rhesus macaque KIR haplotypes show extensive expansions and contractions}

An analysis of a rhesus macaque family comprising thirty animals (Figure 1) revealed the segregation of KIR gene combinations. Twelve previously unreported Mamu-KIR haplotypes were deduced (Figure 3A). All haplotypes were confirmed in multiple animals, except for haplotype $\mathrm{H} 12$, for which material of informative offspring is missing (sire 95055). This deduced haplotype was, however, confirmed by multiple independent PCRs and, in addition, Sanger sequencing to certify low PacBio read counts for the Mamu-KIR3DL2O and KIR3DSO5 genes $(<3$ PacBio reads; Supp. Figure 1A). KIR3DSO5 on haplotype $\mathrm{H} 12$ could not be defined at the allelic level, but the presence was confirmed on gDNA. Furthermore, haplotype $\mathrm{H} 12$ was confirmed in part by a previous study in which the KIR repertoire of sire 95055 was analysed using Sanger sequencing (22).

The number of KIR genes per haplotype showed remarkable variability, and ranged from four to fourteen genes, which produced bona fide transcripts. Mamu-KIR3DL20 was transcribed on all twelve haplotypes, whereas Mamu-KIR3DLO1 was present on all haplotypes except for haplotype H9. These two genes might be considered framework genes in rhesus macaques, although some previously reported macaque KIR haplotypes defined by conventional characterization methodology seem to lack those genes (22). Eight of the twelve Mamu-KIR haplotypes transcribed a Mamu-KIR2DLO4 gene, whereas in humans the orthologous gene is referred to as a framework gene (12). Other common lineage II KIR genes were Mamu-KIR3DLO5, Mamu-KIR3DLO7, MamuKIR3DL08, Mamu-KIR3DL10, Mamu-KIR3DSO2, and Mamu-KIR3DS05, which were 


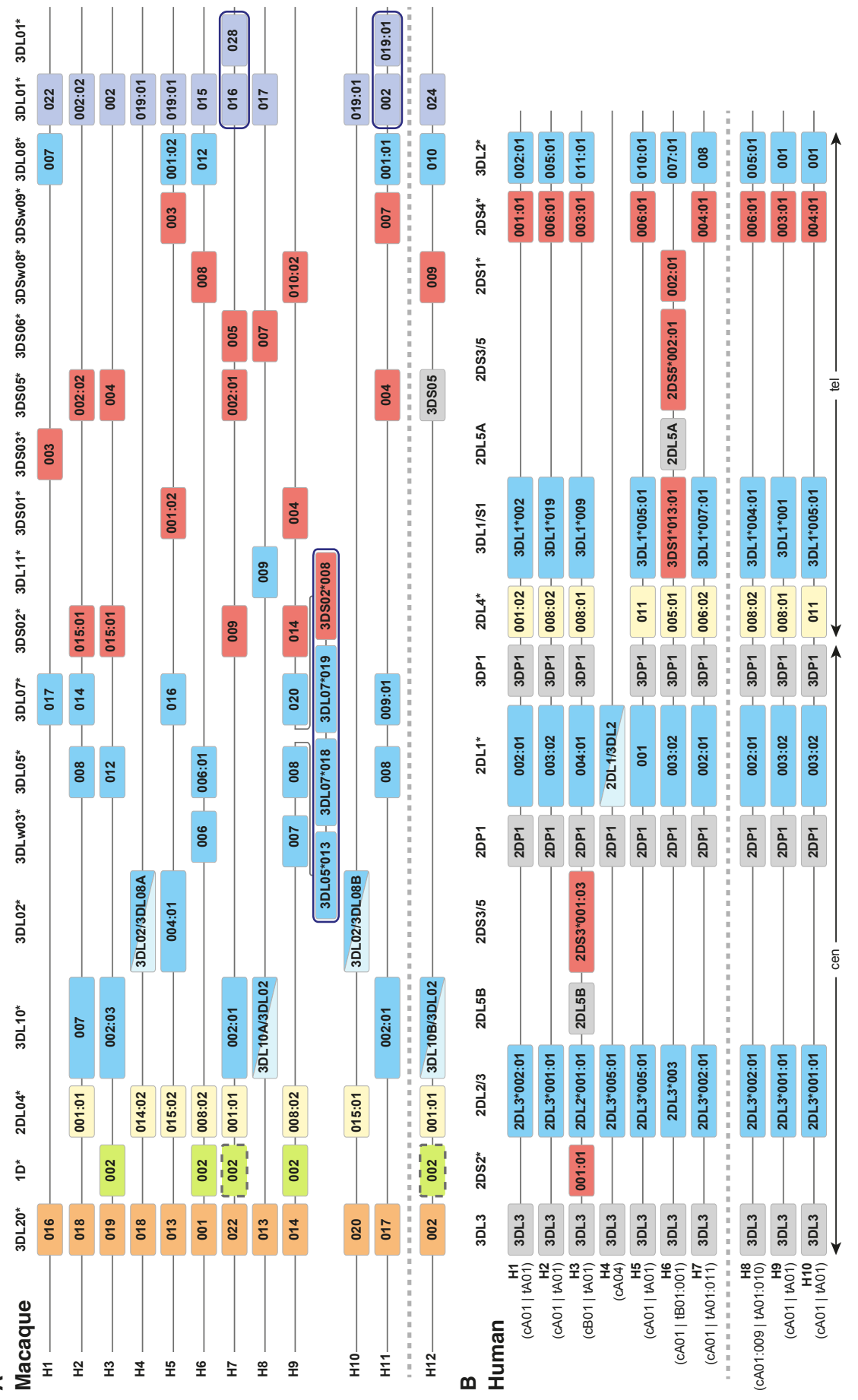


present in $55-70 \%$ of the studied animals. Most of the remaining KIR genes were present in approximately $10-30 \%$ of the animals, or were absent in this family. The more frequently present genes, like Mamu-KIR3DL01, Mamu-KIR3DL05, MamuKIR3DL07, MamU-KIR3DL20, and KIR3DSO2, showed the most allelic variation (Table II), which might indicate a selective pressure on relatively rapidly evolving genes, or that these KIR genes are old entities that accumulated variation over time.

Haplotypes H7, H9, and $\mathrm{H} 11$ showed an expansion of the KIR cluster that was characterized by two or three 'allelic' copies of a certain gene (Figure 3A). On haplotypes H7 and H11, Mamu-KIR3DLO1 was duplicated, resulting in two 'allelic' Mamu-KIR3DLO1 copies that differed at 22 and 12 base pair positions, respectively. Considering the number of SNPs between the Mamu-KIR3DLO1 copies and the fact that they are located on the same chromosome, designating them as different genes could be considered in the future. Macaque haplotype H9 showed a more extensive expansion, with two 'allelic' copies of Mamu-KIR3DLO5 and Mamu-KIR3DSO2, and even three copies of Mamu-KIR3DLO7. Each 'allelic' Mamu-KIR3DLO7 copy varied from the other by at least six SNPs, suggesting that these copies are old entities that accumulated variation over time, and are not the result of recent duplications. The duplicated Mamu-KIR3DLO5, Mamu-KIR3DLO7, and Mamu-KIR3DSO2 copies on haplotype H9 were also confirmed by Sanger sequencing to exclude potential in vitro artefacts generated by the PacBio platform (Supp. Table I and Supp. Figure 1B, C, D).

In contrast to extended haplotypes, macaque haplotypes $\mathrm{H} 4, \mathrm{H} 8, \mathrm{H} 10$, and $\mathrm{H} 12$ seemed to be contracted haplotypes, with only four or seven/eight KIR genes present, including a recombinant gene generated by an in-frame fusion event. All of these contracted haplotypes also contained a Mamu-KIR3DLO1 and Mamu-KIR3DL2O allele along with a few additional lineage I and/or II KIR alleles. The presence of a fusion gene

4 Figure 3. Rhesus macaque and human KIR haplotypes defined on the transcription level. (A) Twelve different $K I R$ haplotypes were characterized by a segregation study involving a large rhesus macaque family. All haplotypes segregate except for haplotype H12, which was only found in sire 95055 and was confirmed by multiple independent PCRs. The physical localization of Mamu-KIR3DL20, Mamu-KIR1D, and Mamu-KIR2DLO4 is based on a completely sequenced macaque KIR haplotype, and these genes are indicated by different colors (27). All other Mamu-KIR3DL genes are indicated in blue boxes and all Mamu-KIR3DS genes in red boxes. Since MamU-KIR3DLO1 is present on all haplotypes except haplotype H9, it might be considered a framework gene, and is therefore indicated by a different blue color. KIR3DSO5 on haplotype H12 (grey box) was determined as present on gDNA, but lacked allele-level typing due to low PacBio read counts ( $<3$ PacBio reads). Gene duplications, or genes that are likely to be inserted as an entity, are indicated by a dark blue outline around the boxes. A recombinant gene, which might be the result of a fusion event, is depicted by a two-colored box (see for instance haplotype $\mathrm{H} 4$ ). On haplotypes $\mathrm{H} 7$ and $\mathrm{H} 12$, it was undeterminable whether KIR1D*002 was present, and these boxes are indicated by a dashed outline. The physical localizations of the Mamu-KIR3DL and the Mamu-KIR3DS genes are predicted based on information from recombinant genes or gene insertions. (B) Ten human KIR haplotypes were characterized. Haplotypes $\mathrm{H} 1$ to $\mathrm{H} 7$ did segregate, whereas haplotypes $\mathrm{H} 8$ to $\mathrm{H} 10$ were only found in a single individual. The inhibitory $K I R(K I R 2 / 3 D L)$ are depicted by blue boxes, whereas the activating KIR (KIR2/3DS) are depicted by red boxes. KIR2DL4 is illustrated by yellow boxes. KIR3DL3 and KIR2DL5 were only determined for their presence or absence on gDNA, and are depicted in grey boxes. The recombinant gene on haplotype $\mathrm{H} 4$ is illustrated by a two-colored box. The gene order and haplotype nomenclature are based on the haplotype conformations as described by Pyo and colleagues (52). 
on all contracted haplotypes might be indicative of recombination events, such as unequal crossing-over and intrachromosomal recombination (Figure 2A, 2B), which might have caused the deletion of genes that were present on the original KIR haplotypes. At least one copy of a recombinant gene was found in $40 \%$ of the animals (Supp. Table II), which might indicate positive selection for contracted haplotypes containing recombinant genes.

The recombination events in macaques may be indicative of the physical position of $K I R$ genes and, in combination with the previously sequenced macaque KIR haplotype by Sambrook and colleagues, the physical locations of the KIR genes were predicted and illustrated in Figure $\mathbf{3 A}$ (27). For example, haplotype $\mathrm{H} 8$ and $\mathrm{H} 12$ contain a recombinant gene with a 5' segment of Mamu-KIR3DL10 and a 3' segment of MamuKIR3DLO2, suggesting that the latter is located downstream of Mamu-KIR3DL10. The recombinant genes on haplotypes $\mathrm{H} 4$ and $\mathrm{H} 10$ suggest that Mamu-KIR3DLO2 should be localized in front of Mamu-KIR3DLO8. Furthermore, Mamu-KIR3DLO5, MamuKIR3DLO7, and Mamu-KIR3DSO2 seemed to have been introduced on macaque haplotype $\mathrm{H} 9$ as a single entity, indicating that these genes were located next to each other, or are at least in close proximity. Nevertheless, to elucidate the precise KIR gene positions, additional genomic haplotype sequencing and phasing needs to be performed.

To confirm that no KIR genes were missed by PCR amplification at the transcription level, the absence or presence of several frequent KIR genes was also determined at the gDNA level (Supp. Table II). This approach suggested that no frequent KIR genes had been missed by the three primer sets used for amplification on CDNA. In addition, although typing for the presence or absence of these genes at the gDNA level might be less informative, in a few cases it provided extra information that was needed to assign them to a certain haplotype.

\section{Identification of full-length cDNA KIR transcripts and a fusion gene in humans}

In humans, one generic primer set, mapping to the UTRs, was able to amplify most KIR2D/3D genes, except for KIR3DL3, KIR2DL4, and KIR2DL5. For KIR2DL4, we designed an additional specific primer set to facilitate analysis of this gene at the transcriptional level (Table I). UTR-specific primers for KIR3DL3 and KIR2DL5 could not be designed, and therefore these genes were only analyzed for the presence or absence at the gDNA level. It is known from the literature that KIR3DL3 is a framework gene, and should be present on all KIR haplotypes, whereas for KIR2DL5, two, one, or no copies can be present only on group $B$ haplotypes $(12,45)$.

A human family consisting of fifteen members was selected, which allowed segregation analysis (Figure 4). The family comprised three generations, and had been founded by five individuals. Approximately 7,900 KIR2D/3D reads and 7,350 KIR2DL4 reads per individual were obtained, and an average of $10-22,5 \%$ of the reads mapped $100 \%$ to the human KIR2D/3D allele library containing 907 reported KIR alleles (14). The remaining reads contained single nucleotide gaps, or were partial sequences introduced by PacBio sequencing. No unreported human KIR alleles were discovered, 


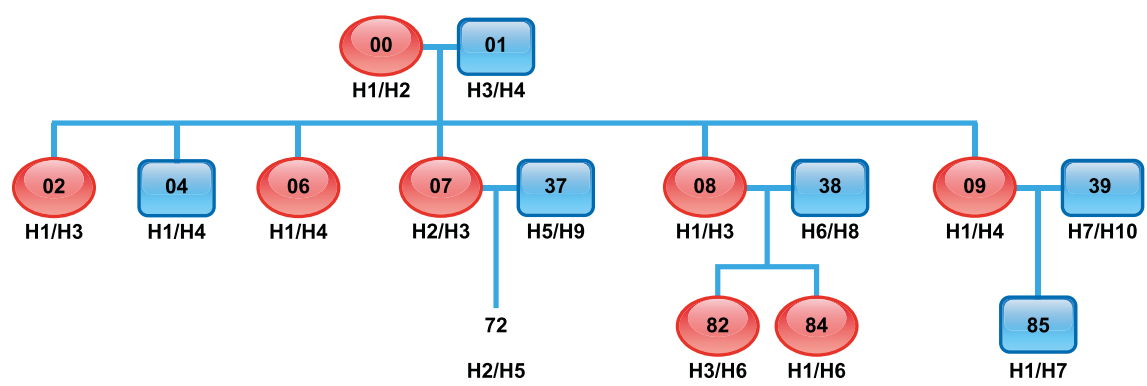

Figure 4. Pedigree of a human family that comprises fifteen individuals. Depicted are the males (blue squares) and the females (red ovals) with their children. The gender of individual 72 was unknown. Different haplotypes are indicated by $\mathrm{H} 1, \mathrm{H} 2$, and so on. In total, ten $\mathrm{KIR}$ haplotypes were identified in this family, of which seven did segregate $(\mathrm{H} 1-\mathrm{H} 7)$ and three were found in a single individual $(\mathrm{H} 8-\mathrm{H} 10)$.

although one known partial sequence was extended (KIR3DL2*011:01, LT934502). Furthermore, a recently reported fusion gene was confirmed, consisting of the $5^{\prime}$ end up to exon 6 of KIR2DL1 and exon 7 to the $3^{\prime}$ end of KIR3DL2 (Figure 2D, LT963640) (42). The fusion transcript encoded a D1-D2 extracellular segment and a long intercellular tail, suggesting an inhibitory KIR2D receptor. Standard KIR typing kits, which are commonly used for $K I R$ characterization in humans, readily miss recombinant $K I R$ genes as they only type for the presence or absence of gene segments at the gDNA level.

\section{On average, less copy number variation for human versus rhesus macaque KIR haplotypes}

Ten KIR haplotypes from fifteen related human individuals were thoroughly defined based on full-length cDNA transcripts (Figure 3B). Each haplotype encoded from three to ten $K I R$ gene transcripts, including representatives of the framework genes $K I R 3 D L 3$, $K I R 2 D L 4$, and KIR3DL2, except for haplotype H4, which lacked a copy of the KIR2DL4 gene. The fourth human framework gene, KIR3DP1, is a pseudo-gene, and is therefore not amplified at the transcription level.

In this communication, we followed the human KIR haplotype nomenclature conventions of Pyo and colleagues, which was later adapted by Vierra-Green and colleagues $(46,47)$. Human haplotypes $\mathrm{H} 1, \mathrm{H} 2, \mathrm{H} 5$, and $\mathrm{H} 7-\mathrm{H} 10$ represent so-called non-variable A haplotypes (cA01|tA01), characterized by six KIR genes (on cDNA level), including the framework genes KIR2DL4 and KIR3DL2, and the activating receptor KIR2DS4. Haplotype H8 was identical to the previously reported cA01:009|tA01:010 haplotype, whereas the telomeric region of $\mathrm{H} 7$ was identical to tA01:011 (46). Group B haplotypes were represented by haplotypes H3 and H6 (Figure 3B), containing up to ten KIR genes, including multiple activating receptors. The telomeric region of haplotype $\mathrm{H} 6$ was identical to the previously reported tB01:001 region, and was combined with a cA01 region (CA01|tB01:001). KIR haplotype H3 confirmed the previously reported cB01|CA01 haplotype configuration (46). Haplotype H4 contained 
only three KIR genes- KIR3DL3, KIR2DL3, and a KIR2DL1/KIR3DL2 fusion gene- the latter suggesting a contracted haplotype. The absence of the framework gene KIR2DL4 on haplotype $\mathrm{H} 4$ might suggest a deletion in the telomeric region of this haplotype. Recently, Roe and colleagues published a contracted haplotype on gDNA that appears to be identical to our haplotype $\mathrm{H} 4$, including the above-described recombinant gene (cA04) (42).

In most human disease association studies, KIR genes are typed by determination of their presence or absence on gDNA. However, multiple studies demonstrated that health and disease could be linked to certain KIR alleles instead of to KIR genes (48-50). Therefore, high-resolution characterization of the KIR genes might be clinically beneficial, and could improve future KIR disease association studies. To confirm that our transcriptome characterization approach did amplify all KIR genes, their presence or absence was also assayed at the gDNA level using the Olerup SSP ${ }^{\circledR}$ KIR typing kit. All seventeen KIR genes that were identified as present on gDNA were also detected at the transcription level, except for KIR2DL5, KIR3DL3, and the pseudo-genes (Supp. Table III). The pseudo-genes KIR3DP1 and KIR2DP1 were present in all genotyped individuals. KIR3DP1 is a framework gene and was suggested to be present on all haplotypes, except for haplotype $\mathrm{H} 4$, which is assumed to lack the KIR3DP1 gene due to a deletion in the telomeric region as described by Roe and colleagues (Figure $\mathbf{3 B}$ ) (42). KIR2DP1 has been described to be present on CA01 and CB01 regions (46), suggesting the presence of this pseudo-gene on all haplotypes of the studied human family. The framework gene KIR3DL3 and the group B haplotype-specific KIR2DL5 were found present at gDNA level in all individuals and on both group $B$ haplotypes $\mathrm{H} 3$ and H6, respectively (Figure 3B; grey boxes). These findings supported the assumption that our transcriptome protocol amplified all KIR genes, except for KIR2DL5, KIR3DL3, and the pseudo-genes, and might be beneficial for future KIR disease association studies. In addition, the identification of multiple human KIR haplotype regions that had been reported by others further validated our protocol.

\section{Discussion}

More recently, sequencing and characterization studies provided insights into the complexity of the KIR gene system. However, due to large gene copy number variation and to the high similarity of the KIR genes, conventional sequencing methods hampered the accurate characterization of the complete KIR system at the transcription level. Here, we describe a comprehensive and relatively fast SMRT sequencing protocol using a PacBio Sequel platform to completely characterize the KIR transcriptomes in human and rhesus macaque families. The power of this approach is demonstrated by the fact that, in a relatively short time, novel KIR genes, alleles, and complex KIR haplotypes were defined by segregation studies in a family set-up. A relatively high number of human and macaque recombinant KIR genes were discovered, and seemed to be the result of several independent fusion events. This study allowed comparison of the 
human and rhesus macaque KIR transcriptomes. Eventually, this may result in a better understanding and interpretation of KIR disease association studies.

A comparison of ten human and twelve rhesus macaque KIR haplotypes illustrates that both species share highly similar gene systems. Although there are subtle differences, such as different receptor lineage expansion and haplotype organisation, both species show extensive allelic polymorphism and gene copy number variation in their KIR repertoire. Hence, rhesus macaques may provide relevant models to study the impact of KIR genes on health and disease. Human KIR allelic polymorphism seems to have already been broadly mapped, as all the alleles we recovered are documented in a database containing over 900 alleles extracted from numerous population studies (14). In contrast, only 218 Mamu-KIR alleles were reported, but considering that almost half of the total Mamu-KIR alleles discovered in this study were unreported, it is reasonable to suggest that this number is only the tip of the iceberg. Therefore, the extent of allelic KIR polymorphism seems to be at least comparable in humans and rhesus macaques.

KIR gene copy number variation is observed on the human and rhesus macaque haplotypes, which contained from four to twelve or four to fourteen KIR (pseudo-) genes, respectively. In humans, the non-variable group A haplotypes rarely show copy number variation, whereas the group $B$ haplotypes can have a variable number of mainly activating - KIR genes, in part caused by duplications or chromosomal rearrangements (Figure 3B). In macaques, each haplotype can contain a different number of inhibitory and activating KIR genes, which can be magnified by deletions or insertions as a result of duplications and chromosomal rearrangements (Figure $\mathbf{3 A}$ ). In comparison to the human situation, the overall CNV seems to be more extensive in the macaque KIR gene system, and might be explained by co-evolution with the expanded $\mathrm{MHC}$ class I repertoire in macaques, and by the absence of a haplotypic organisation as is observed in humans.

One human and four macaque haplotypes showed signs of contraction. In both species, the contraction of haplotypes was marked by the presence of recombinant genes and the apparent deletion of a haplotype segment. The generation of these contracted haplotypes is most likely mediated by repetitive elements present in the KIR introns (43). For some human recombinant haplotypes, these sequence repeats are identified and characterized as breakpoints that may facilitate chromosomal rearrangements (Figure 2A, B). In macaques, however, these repetitive elements are not yet characterized, but considering the observation of short Mamu-KIR haplotypes, in addition to the presence of recombinant genes, it is likely that the same mechanisms are responsible for $K I R$ gene expansion and haplotype contraction as is observed in humans. The rapid loss and gain of KIR genes, driven by the repetitive sequence elements in the introns, might be an advantageous evolutionary strategy to expand gene variability using an existing gene repertoire, and thereby enhancing pathogen evasion. Hence, recombinant genes are composed of different heads (ligand interaction) and tails (signaling function), which may facilitate the exchange of functionalities and ligand interactions between receptors (Figure $\mathbf{2 C}, \mathbf{D}$ ). 
In macaques, in addition to contraction, expansion of KIR haplotypes was also observed. Macaque haplotypes $\mathrm{H} 7$ and $\mathrm{H} 11$ seem to have expanded by gene duplication, whereas haplotype $\mathrm{H} 9$ showed evidence of unequal crossing-over events. However, it is arguable whether the duplication on macaque haplotypes $\mathrm{H} 7$ and $\mathrm{H} 11$ should be considered as copies of a Mamu-KIR3DLO1 gene, since the 'allelic' copies vary at 22 and 12 base pair positions, respectively. A sensible nomenclature system for Mamu-KIR3DLO1 sequences should be considered. A more extreme expansion is observed on macaque haplotype H9, which contains two 'allelic' copies of MamuKIR3DLO5 and Mamu-KIR3DSO2 and three Mamu-KIR3DLO7 copies. Although it is possible that this expansion is explained by multiple gene duplications, it seems more likely that these three duplicated genes are introduced as one entity or tandem, on which Mamu-KIR3DLO7 might already have been duplicated.

Although no expanded human KIR haplotypes were found in the present human family studied, other researchers reported $K I R$ haplotypes with gene insertions, similar to the extended macaque haplotype $H 9(42,51,52)$.

The expansion and contraction of $K I R$ haplotypes might be a balancing selection for fighting infections on the one hand and for reproductive success on the other. A similar reproductive/immunological trade-off is illustrated by the haplotypic organization in human KIR (12). In humans, expansion and contraction of the KIR region is only observed on group $B$ haplotypes, which indicates that structurally diversifying the nonvariable group A haplotypes is not beneficial. The group B haplotypes, which can contain a variable number of $K I R$ genes, show chromosomal rearrangements, whereby $K I R$ genes are introduced and/or deleted. This diversifying selection might be associated with increasing immune response variability, successful reproduction, loss of unfavourable genes, or the generation of novel fusion genes. However, chromosomal recombination might also be driven by the specific content of group $B$ haplotypes. In this case, sequence elements that are present on group B haplotype-specific genes, such as activating KIR and KIR2DL5, might drive recombination events, without necessarily requiring selective pressure. In macaques, as well as in all other non-human primates, there is no haplotypic organization that divides variable and non-variable haplotype content. All macaque haplotypes seem to be prone to diversifying selection, as great variability in gene content is observed, but association with reproductive success or pathogen evasion has not yet been demonstrated. On an individual level, contracted haplotypes should provide the essential functions of the KIR gene system. Mamu-KIR3DLO1 and Mamu-KIR3DL2O are two highly polymorphic and frequently expressed genes, and all four contracted Mamu-KIR haplotypes identified in this study have expression of these genes in common. Three of the studied macaques (R12035, R13084, R14072) are homozygous for contracted haplotypes (Figs. 1, 4A), and do not show signs of an impaired immune system. This might indicate that Mamu-KIR3DLO1 and Mamu-KIR3DL2O expression, in combination with a recombinant gene, is sufficient to provide functional NK cell activity.

Previously, two population studies $(51,52)$ and two smaller studies $(42,53)$ reported contracted and expanded KIR haplotypes at the gDNA level in humans. In three of 
these studies, almost twice as many contracted haplotypes were observed in comparison to expanded haplotypes. However, the prevalence of contracted and expanded haplotypes in these populations with European ancestry was only $5-10 \%$. In the selected rhesus macaque family, four of the twelve Mamu-KIR haplotypes were contracted, whereas only one showed extensive expansion. In $46 \%$ of the animals of the studied rhesus macaque family, a contracted or expanded haplotype was observed. Although a haplotype analysis of a larger rhesus macaque population is required to compare the prevalence of contraction and expansion to that observed in humans, this study suggests that chromosomal rearrangements in the KIR cluster is more common in rhesus macaques than it is in humans. Furthermore, the results indicate that contraction, accompanied by the generation of novel recombinant genes, seems to be more beneficial, or at least has a higher occurrence, than expansion of KIR haplotypes.

In the past, different typing strategies were reported to characterize the KIR cluster in humans and rhesus macaques, including cloning and Sanger sequencing, Roche/454 pyro-sequencing, and microsatellite analysis (22-24, 27, 33, 38). However, these methods were often insufficient to assemble full-length allele sequences, as well as being time consuming and mainly focused on the presence or absence of genes. More recent reports described high-resolution KIR characterization on gDNA by NextGeneration Sequencing (NGS) on a PacBio RS II platform, a MiSeq platform, or by exome capturing $(42,54,55)$. These studies were able to define 'allelic'-level genotypes and to identify novel alleles, and less frequently, defined complete or partial haplotypes, and discovered recombinant genes at the gDNA level. Another recent study used SMRT sequencing on a PacBio RS II platform to characterize KIR transcription in an inbred Mauritian cynomolgus macaque population, which is restricted in its genetic diversity, and was able to identify nine novel alleles and to define KIR haplotypes (56). Our studies show that a similar approach is also applicable to outbred human and macaque populations.

Most current clinical methods to characterize the KIR gene system are primarily based on determining the presence or absence of the known human KIR gene segments, and they might miss substantial information such as allele-level typing, copy number variation, expanded haplotypes, and recombinant genes. Multiple disease association studies, however, illustrated the importance of distinguishing between alleles of KIR genes (48-50). Therefore, a high-resolution KIR characterization approach might be beneficial for future health and disease studies.

In this communication, a method is described to thoroughly characterize the KIR transcriptomes in humans and rhesus macaques, using a relatively fast high-resolution SMRT sequencing protocol on a PacBio Sequel platform. Novel alleles and recombinant genes were discovered, and transcribed haplotypes were defined based on transcription profiles in concert with segregation studies in families. Although sequencing at the transcription level might have minor drawbacks, such as the lack of intron and pseudogene information, it does have serious advantages over sequencing on gDNA; for example, transcriptional modifications can be observed, including splicing of transcripts or intron insertions. These transcriptional modifications might have an effect on the 
function of the receptors; for instance, when KIR3D transcripts can be spliced to generate KIR2D transcripts, or when transmembrane regions are spliced out, which may result in soluble receptors. In addition, although the PacBio platform does not yet provide quantitative analysis, the number of identified transcripts might be indicative of the expression level of a certain allele. However, this indication might be affected by other factors, such as variegated KIR expression, the MHC class I gene repertoire, and previous pathogen exposure.

In conclusion, this comprehensive sequencing approach can eventually contribute to better understanding and characterizing the KIR gene cluster in different species, thereby improving not only the interpretation of disease association studies but also transplantation and reproduction biology.

\section{Acknowledgements}

We thank D. Devine for editing the manuscript and F. van Hassel for preparing the figures. 


\section{References}

1. Mingari, M. C., A. Moretta, and L. Moretta. 1998. Regulation of KIR expression in human T cells: a safety mechanism that may impair protective T-cell responses. Immunol Today. 19: 153-157.

2. Trowsdale, J. 2001. Genetic and functional relationships between MHC and NK receptor genes. Immunity. 15: 363-374.

3. Parham, P., P. J. Norman, L. Abi-Rached, and L. A. Guethlein. 2012. Human-specific evolution of killer cell immunoglobulin-like receptor recognition of major histocompatibility complex class I molecules. Philos Trans $R$ Soc Lond B Biol Sci. 367: 800-811.

4. Parham, P., and T. Ohta. 1996. Population biology of antigen presentation by MHC class I molecules. Science. 272: $67-74$

5. Khakoo, S. I., and M. Carrington. 2006. KIR and disease: a model system or system of models? Immunological reviews. 214: 186-201.

6. Purdy, A. K., and K. S. Campbell. 2009. Natural killer cells and cancer: regulation by the killer cell Ig-like receptors (KIR). Cancer biology \& therapy. 8: 2211-2220.

7. Hershberger, K. L., R. Shyam, A. Miura, and N. L. Letvin. 2001. Diversity of the killer cell Ig-like receptors of rhesus monkeys. Journal of immunology (Baltimore, Md : 1950). 166: 4380-4390.

8. Bimber, B. N., and D. T. Evans. 2015. The killer-cell immunoglobulin-like receptors of macaques. Immunological reviews. 267: 246-258.

9. Marsh, S. G., P. Parham, B. Dupont, D. E. Geraghty, J. Trowsdale, D. Middleton, C. Vilches, M. Carrington, C. Witt, L. A. Guethlein, H. Shilling, C. A. Garcia, K. C. Hsu, and H. Wain. 2003. Killer-cell immunoglobulin-like receptor (KIR) nomenclature report, 2002. Human immunology. 64: 648-654.

10. Trowsdale, J., R. Barten, A. Haude, C. A. Stewart, S. Beck, and M. J. Wilson. 2001. The genomic context of natural killer receptor extended gene families. Immunological reviews. 181: 20-38.

11. Hsu, K. C., S. Chida, D. E. Geraghty, and B. Dupont. 2002. The killer cell immunoglobulin-like receptor (KIR) genomic region: gene-order, haplotypes and allelic polymorphism. Immunological reviews. 190: 40-52.

12. Wilson, M. J., M. Torkar, A. Haude, S. Milne, T. Jones, D. Sheer, S. Beck, and J. Trowsdale. 2000. Plasticity in the organization and sequences of human KIR/ILT gene families. Proceedings of the National Academy of Sciences of the United States of America. 97: 4778-4783.

13. Uhrberg, M., N. M. Valiante, B. P. Shum, H. G. Shilling, K. Lienert-Weidenbach, B. Corliss, D. Tyan, L. L. Lanier, and P. Parham. 1997. Human diversity in killer cell inhibitory receptor genes. Immunity. 7: 753-763.

14. Robinson, J., K. Mistry, H. McWilliam, R. Lopez, and S. G. E. Marsh. 2010. IPD-the Immuno Polymorphism Database. Nucleic Acids Research. 38: D863-869.

15. Giebel, S., F. Locatelli, T. Lamparelli, A. Velardi, S. Davies, G. Frumento, R. Maccario, F. Bonetti, J. Wojnar, M. Martinetti, F. Frassoni, G. Giorgiani, A. Bacigalupo, and J. Holowiecki. 2003. Survival advantage with KIR ligand incompatibility in hematopoietic stem cell transplantation from unrelated donors. Blood. 102: 814-819.

16. Hiby, S. E., J. J. Walker, M. O'Shaughnessy K, C. W. Redman, M. Carrington, J. Trowsdale, and A. Moffett. 2004. Combinations of maternal KIR and fetal HLA-C genes influence the risk of preeclampsia and reproductive success. The Journal of experimental medicine. 200: 957-965.

17. Abi-Rached, L., and P. Parham. 2005. Natural selection drives recurrent formation of activating killer cell immunoglobulin-like receptor and Ly49 from inhibitory homologues. The Journal of experimental medicine. 201: 1319-1332.

18. Kelley, J., L. Walter, and J. Trowsdale. 2005. Comparative Genomics of Natural Killer Cell Receptor Gene Clusters. PLOS Genetics. 1:

19. Kaushal, D., S. Mehra, P. Didier, and A. Lackner. 2012. The non-human primate model of tuberculosis. Journal of medical primatology. 41: 191-201.

20. Vierboom, M. P., M. Jonker, R. E. Bontrop, and B. t Hart. 2005. Modeling human arthritic diseases in nonhuman primates. Arthritis research \& therapy. 7: 145-154.

21. Bontrop, R. E. 2001. Non-human primates: essential partners in biomedical research. Immunological reviews. 183: 5-9. 
22. Blokhuis, J. H., M. K. van der Wiel, G. G. Doxiadis, and R. E. Bontrop. 2010. The mosaic of KIR haplotypes in rhesus macaques. Immunogenetics. 62: 295-306.

23. Blokhuis, J. H., M. K. van der Wiel, G. G. Doxiadis, and R. E. Bontrop. 2011. The extreme plasticity of killer cell Iglike receptor (KIR) haplotypes differentiates rhesus macaques from humans. European journal of immunology. 41: $2719-2728$.

24. Moreland, A. J., L. A. Guethlein, R. K. Reeves, K. W. Broman, R. P. Johnson, P. Parham, D. H. O'Connor, and B. N. Bimber. 2011. Characterization of killer immunoglobulin-like receptor genetics and comprehensive genotyping by pyrosequencing in rhesus macaques. BMC genomics. 12: 295.

25. Otting, N., C. M. Heijmans, R. C. Noort, N. G. de Groot, G. G. Doxiadis, J. J. van Rood, D. I. Watkins, and R. E. Bontrop. 2005. Unparalleled complexity of the $\mathrm{MHC}$ class I region in rhesus macaques. Proceedings of the National Academy of Sciences of the United States of America. 102: 1626-1631.

26. de Groot, N. G., J. H. Blokhuis, N. Otting, G. G. Doxiadis, and R. E. Bontrop. 2015. Co-evolution of the MHC class I and KIR gene families in rhesus macaques: ancestry and plasticity. Immunological reviews. 267: 228-245.

27. Sambrook, J. G., A. Bashirova, S. Palmer, S. Sims, J. Trowsdale, L. Abi-Rached, P. Parham, M. Carrington, and S. Beck. 2005. Single haplotype analysis demonstrates rapid evolution of the killer immunoglobulin-like receptor (KIR) loci in primates. Genome research. 15: 25-35.

28. Blokhuis, J. H., G. G. Doxiadis, and R. E. Bontrop. 2009. A splice site mutation converts an inhibitory killer cell Ig-like receptor into an activating one. Molecular immunology. 46: 640-648.

29. Colantonio, A. D., B. N. Bimber, W. J. Neidermyer, Jr., R. K. Reeves, G. Alter, M. Altfeld, R. P. Johnson, M. Carrington, D. H. O'Connor, and D. T. Evans. 2011. KIR polymorphisms modulate peptide-dependent binding to an MHC class I ligand with a Bw6 motif. PLoS pathogens. 7: e1001316.

30. Andersen, H., J. L. Rossio, V. Coalter, B. Poore, M. P. Martin, M. Carrington, and J. D. Lifson. 2004. Characterization of rhesus macaque natural killer activity against a rhesus-derived target cell line at the singlecell level. Cellular immunology. 231: 85-95.

31. Grendell, R. L., A. L. Hughes, and T. G. Golos. 2001. Cloning of rhesus monkey killer-cell Ig-like receptors (KIRs) from early pregnancy decidua. Tissue antigens. 58: 329-334.

32. Bostik, P., J. Kobkitjaroen, W. Tang, F. Villinger, L. E. Pereira, D. M. Little, S. T. Stephenson, M. Bouzyk, and A. A. Ansari. 2009. Decreased NK cell frequency and function is associated with increased risk of KIR3DL allele polymorphism in simian immunodeficiency virus-infected rhesus macaques with high viral loads. Journal of immunology (Baltimore, Md : 1950). 182: 3638-3649.

33. Kruse, P. H., C. Rosner, and L. Walter. 2010. Characterization of rhesus macaque KIR genotypes and haplotypes. Immunogenetics. 62: 281-293.

34. Chaichompoo, P., P. Bostik, S. Stephenson, S. Udompunturuk, J. Kobkitjaroen, K. Pattanapanyasat, and A. A. Ansari. 2010. Multiple KIR gene polymorphisms are associated with plasma viral loads in SIV-infected rhesus macaques. Cellular immunology. 263: 176-187.

35. Hellmann, I., S. Y. Lim, R. S. Gelman, and N. L. Letvin. 2011. Association of activating KIR copy number variation of NK cells with containment of SIV replication in rhesus monkeys. PLoS pathogens. 7: e1002436.

36. PacBio website [http://www.pacb.com]

37. Kearse, M., R. Moir, A. Wilson, S. Stones-Havas, M. Cheung, S. Sturrock, S. Buxton, A. Cooper, S. Markowitz, C. Duran, T. Thierer, B. Ashton, P. Meintjes, and A. Drummond. 2012. Geneious Basic: an integrated and extendable desktop software platform for the organization and analysis of sequence data. Bioinformatics (Oxford, England). 28: 1647-1649.

38. Bimber, B. N., A. J. Moreland, R. W. Wiseman, A. L. Hughes, and D. H. O'Connor. 2008. Complete Characterization of Killer Ig-Like Receptor (KIR) Haplotypes in Mauritian Cynomolgus Macaques: Novel Insights into Nonhuman Primate KIR Gene Content and Organization. Journal of immunology (Baltimore, Md : 1950). 181: 6301-6308.

39. Rhoads, A., and K. F. Au. 2015. PacBio Sequencing and Its Applications. Genomics, proteomics \& bioinformatics. 13: $278-289$

40. Westbrook, C. J., J. A. Karl, R. W. Wiseman, S. Mate, G. Koroleva, K. Garcia, M. Sanchez-Lockhart, D. H. O'Connor, and G. Palacios. 2015. No assembly required: Full-length MHC class I allele discovery by PacBio circular consensus sequencing. Human immunology. 76: 891-896. 
41. Martin, M. P., A. Bashirova, J. Traherne, J. Trowsdale, and M. Carrington. 2003. Cutting Edge: Expansion of the KIR Locus by Unequal Crossing Over. The Journal of Immunology. 171: 2192-2195.

42. Roe, D., C. Vierra-Green, C. W. Pyo, K. Eng, R. Hall, R. Kuang, S. Spellman, S. Ranade, D. E. Geraghty, and M. Maiers. 2017. Revealing complete complex KIR haplotypes phased by long-read sequencing technology. Genes and immunity.

43. Traherne, J. A., M. Martin, R. Ward, M. Ohashi, F. Pellett, D. Gladman, D. Middleton, M. Carrington, and J. Trowsdale. 2010. Mechanisms of copy number variation and hybrid gene formation in the KIR immune gene complex. Human molecular genetics. 19: 737-751.

44. Ordonez, D., N. Gomez-Lozano, L. Rosales, and C. Vilches. 2011. Molecular characterisation of KIR2DS2*005, a fusion gene associated with a shortened KIR haplotype. Genes and immunity. 12: 544-551.

45. Cisneros, E., M. Moraru, N. Gomez-Lozano, M. Lopez-Botet, and C. Vilches. 2012. KIR2DL5: An Orphan Inhibitory Receptor Displaying Complex Patterns of Polymorphism and Expression. Frontiers in immunology. 3 : 289.

46. Pyo, C. W., L. A. Guethlein, Q. Vu, R. Wang, L. Abi-Rached, P. J. Norman, S. G. Marsh, J. S. Miller, P. Parham, and D. E. Geraghty. 2010. Different patterns of evolution in the centromeric and telomeric regions of group $A$ and $B$ haplotypes of the human killer cell Ig-like receptor locus. PloS one. 5: e15115.

47. Vierra-Green, C., D. Roe, L. Hou, C. K. Hurley, R. Rajalingam, E. Reed, T. Lebedeva, N. Yu, M. Stewart, H. Noreen, J. A. Hollenbach, L. A. Guethlein, T. Wang, S. Spellman, and M. Maiers. 2012. Allele-Level Haplotype Frequencies and Pairwise Linkage Disequilibrium for 14 KIR Loci in 506 European-American Individuals. PloS one. 7:

48. Bari, R., P. Rujkijyanont, E. Sullivan, G. Kang, V. Turner, K. Gan, and W. Leung. 2013. Effect of Donor KIR2DL1 Allelic Polymorphism on the Outcome of Pediatric Allogeneic Hematopoietic Stem-Cell Transplantation. Journal of Clinical Oncology. 31: 3782-3790

49. Martin, M. P., Y. Qi, X. Gao, E. Yamada, J. N. Martin, F. Pereyra, S. Colombo, E. E. Brown, W. L. Shupert, J. Phair, J. J. Goedert, S. Buchbinder, G. D. Kirk, A. Telenti, M. Connors, S. J. O’Brien, B. D. Walker, P. Parham, S. G. Deeks, D. W. McVicar, and M. Carrington. 2007. Innate partnership of HLA-B and KIR3DL1 subtypes against HIV-1. Nature genetics. 39: 733-740.

50. Berinstein, J., R. Pollock, F. Pellett, A. Thavaneswaran, V. Chandran, and D. D. Gladman. 2017. Association of variably expressed KIR3dl1 alleles with psoriatic disease. Clinical rheumatology.

51. Jiang, W., C. Johnson, J. Jayaraman, N. Simecek, J. Noble, M. F. Moffatt, W. O. Cookson, J. Trowsdale, and J. A Traherne. 2012. Copy number variation leads to considerable diversity for B but not A haplotypes of the human KIR genes encoding NK cell receptors. Genome research. 22: 1845-1854.

52. Pyo, C. W., R. Wang, Q. Vu, N. Cereb, S. Y. Yang, F. M. Duh, S. Wolinsky, M. P. Martin, M. Carrington, and D. E. Geraghty. 2013. Recombinant structures expand and contract inter and intragenic diversification at the KIR locus. BMC genomics. 14: 89.

53. Hou, L., M. Chen, J. Ng, and C. K. Hurley. 2012. Conserved KIR allele-level haplotypes are altered by microvariation in individuals with European ancestry. Genes and immunity. 13: 47-58.

54. Maniangou, B., N. Legrand, M. Alizadeh, U. Guyet, C. Willem, G. David, E. Charpentier, A. Walencik, C. Retiere, and K. Gagne. 2017. Killer Immunoglobulin-Like Receptor Allele Determination Using Next-Generation Sequencing Technology. Frontiers in immunology. 8: 547

55. Norman, P. J., J. A. Hollenbach, N. Nemat-Gorgani, W. M. Marin, S. J. Norberg, E. Ashouri, J. Jayaraman, E. E. Wroblewski, J. Trowsdale, R. Rajalingam, J. R. Oksenberg, J. Chiaroni, L. A. Guethlein, J. A. Traherne, M. Ronaghi, and P. Parham. 2016. Defining KIR and HLA Class I Genotypes at Highest Resolution via High-Throughput Sequencing. American journal of human genetics. 99: 375-391.

56. Prall, T. M., M. E. Graham, J. A. Karl, R. W. Wiseman, A. J. Ericsen, M. Raveendran, R. Alan Harris, D. M. Muzny, R. A. Gibbs, J. Rogers, and D. H. O'Connor. 2017. Improved full-length killer cell immunoglobulin-like receptor transcript discovery in Mauritian cynomolgus macaques. Immunogenetics. 69: 325-339. 


\section{Supplementary materials}

\section{A}

Mamu-KIR3DL20002 PacBio
Mamu-KLR3DL20001 PacBio Mamu-KIR3DL20*01 PacBio

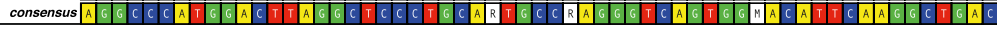

Mamu-KIR3DL20*001/002

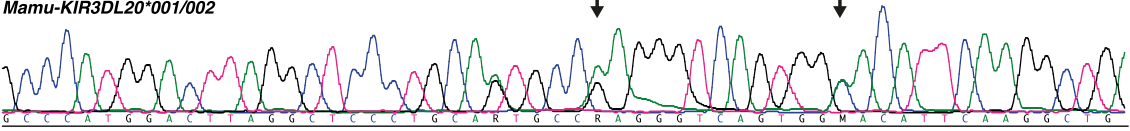

B

Mamu-KIR3DL05*008 PacBio Mamu-KIR3DL05*008 Sanger Mamu-KIR3DL05*013 PacBio Mamu-KIR3DL05*013 Sanger

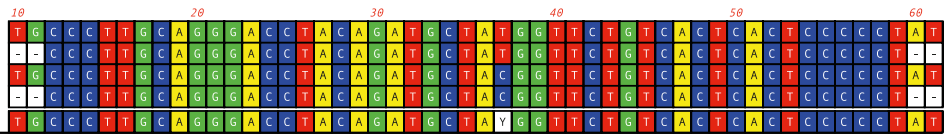

Mamu-KIR3DLO5*008

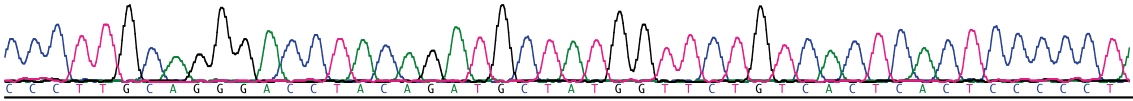

Mamu-KIR3DL05*013

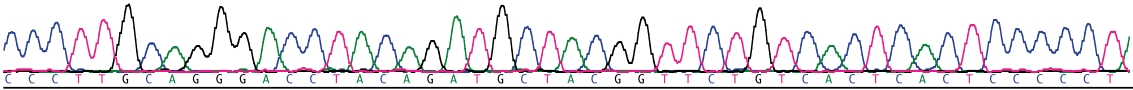

C

3DL07*018_JBR PacBio TO TC A G G G A

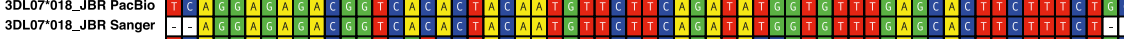
3DL07*019_JBR PacBio

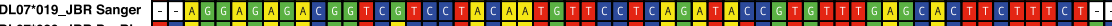

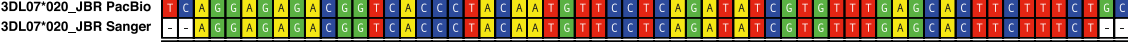

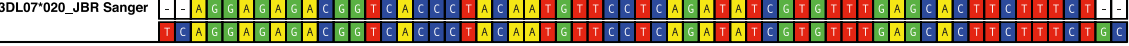

Mamu-KIR3DLO7*018

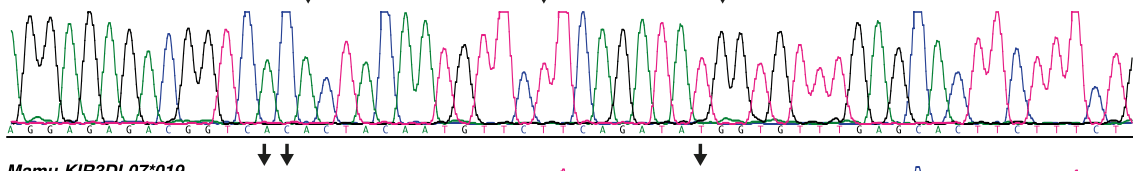
Mamu-KIR3DL07*019

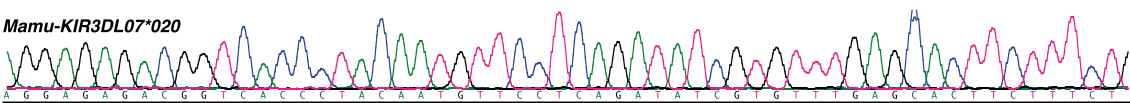

D

Mamu-KIR3DS02*008 PacBio G A

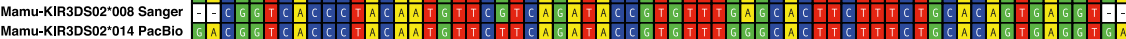

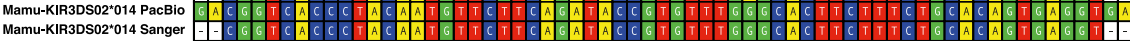
Mamu-KIR3DS02*014 Sanger
consensus

Mamu-KIR3DS02*008

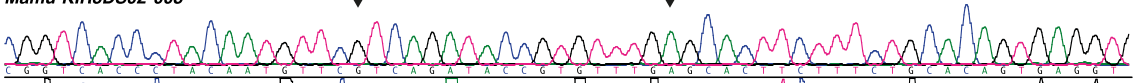

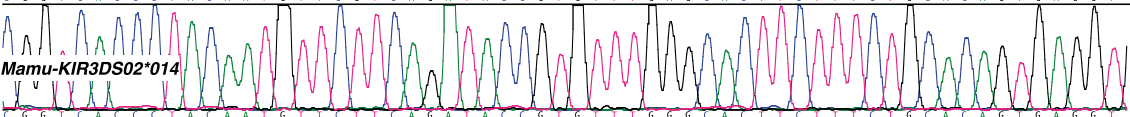


Supplementary Figure 1. Sequence confirmation by Sanger sequencing. Chromatograms and sequences obtained by Sanger sequencing to confirm results found by PacBio sequencing. Arrows in the chromatograms indicate sequence variation. Primers used for Sanger sequencing are listed in Supp. Table I. (A) Macaque haplotype $\mathrm{H} 12$ lacked informative offspring, and was therefore only identified in a single sire (95055). The haplotype was confirmed by multiple independent PCRs. Nonetheless, low PacBio read counts were obtained from the Mamu-KIR3DL20 alleles, and therefore these alleles were confirmed by Sanger sequencing. The arrows indicate double peaks, illustrating the presence of Mamu-KIR3DL20*001 and Mamu-KIR3DL20*002 in sire 95055, which allowed us to assign the alleles to the specific haplotypes. (B) Two copies of the MamuKIR3DL05 gene were found on macaque haplotype H9. One of these alleles was novel (Mamu-KIR3DL05*013), whereas the other was reported previously (Mamu-KIR3DL05*008).(C) Three copies of the Mamu-KIR3DL07 gene were found on macaque haplotype H9. These alleles were all novels, and confirmed by Sanger sequencing. (D) Two copies of the Mamu-KIR3DSO2 gene were found on macaque haplotype H9. One of these alleles was novel (Mamu-KIR3DS02*014), whereas the other was reported previously (Mamu-KIR3DS02*008).

Supplementary table I. Overview of forward $(\mathrm{Fw})$ and reverse (Rv) primers used to confirm alleles on macaque and human KIR haplotypes. To amplify different MamU-KIR3DLO7 alleles, a wobble had to be introduced in the forward primer sequence: adenine or thymine (W). The PCR product sizes were sufficient to distinguish alleles and confirm their presence.

\begin{tabular}{|c|c|c|c|c|}
\hline Target & Fw / Rv & Exon & Primer sequence 5' - 3' & Product size \\
\hline \multirow[t]{2}{*}{ Mamu-KIR3DLO5 } & Fw & 4 & TCTGCAAAGTGAGGTGACCTT & 337 bp \\
\hline & Rv & 5 & AAGCCTAAGTTCATGGGTCTCC & \\
\hline \multirow[t]{2}{*}{ Mamu-KIR3DLO7 } & $\mathrm{FW}$ & 3 & GACCTTCTTGWTTGCCCGGC & 556 bp \\
\hline & Rv & 4 & ACTGGGAGCTGACAACACATAGTC & \\
\hline \multirow[t]{2}{*}{ Mamu-KIR3DSO2 } & $\mathrm{Fw}$ & 3 & GATCATACCCGCACTCCCCAA & $319 \mathrm{bp}$ \\
\hline & $\mathrm{Rv}$ & 4 & TGGGAGTGAGTGACAGAACCA & \\
\hline \multirow[t]{2}{*}{ Mamu-KIR3DL2O } & Fw & 5 & CCAGAGCTCGTTTGACATTTACCG & $183 \mathrm{bp}$ \\
\hline & Rv & 5 & GGGTGTGACCACACATAGGGCAG & \\
\hline \multirow[t]{2}{*}{ Mamu-KIR3DSO5 } & $\mathrm{FW}$ & 5 & GTGTCAACGGAACATTCCAGGA & $109 \mathrm{bp}$ \\
\hline & Rv & 5 & CTCGGGTGTGACCACTTGTAGGA & \\
\hline \multirow[t]{2}{*}{ Human KIR2DL1 } & $\mathrm{FW}$ & 3 & АСTTTGCGCCTCATTGGAGA & $359 \mathrm{bp}$ \\
\hline & $\mathrm{Rv}$ & 4 & CCCAGAGGAAAGTCAGCCTG & \\
\hline \multirow[t]{2}{*}{ Human KIR2DL4 } & $\mathrm{FW}$ & 9 & GCCCTTCTCAGAGGAGCAAG & $116 \mathrm{bp}$ \\
\hline & Rv & 9 & CATCAAGGCCTGACTGTGGT & \\
\hline \multirow[t]{2}{*}{ Human KIR2DS4 } & Fw & 4 & GGTTCAGGCAGGAGAGAAT & $111 / 133 b p$ \\
\hline & $\mathrm{Rv}$ & 4 & CTGGAATGTTCCGTKGATG & \\
\hline
\end{tabular}

Supplementary table II \& III. These tables are too large to be readable in this thesis. We therefore chose to make them available online as a PDF file. We recommend to display them on a computer screen. Please use the QR-codes or type the link address in your browser window.

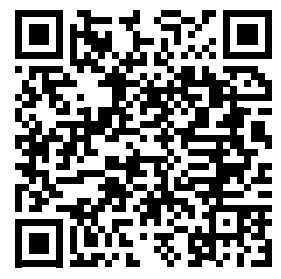

Supplementary table II

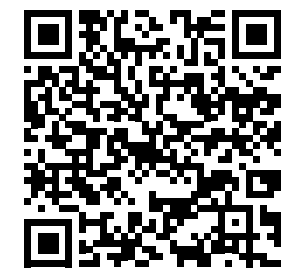

Supplementary table III
Supplementary table II

https://www.bprc.nl/sites/default/files/ downloads/thesis/JB-figS02.pdf

Supplementary table III https://www.bprc.nl/sites/default/files/ downloads/thesis/JB-figS03.pdf 



\section{Chapter \\ Unparalleled Rapid Evolution of $K I R$ Genes in Rhesus and Cynomolgus Macaque Populations}

Jesse Bruijnesteijn ${ }^{1}$, Nanine de Groot ${ }^{1}$, Marit K. H. van der Wiel $^{1}$, Nel Otting ${ }^{1}$, Annemiek J. M. de Vos-Rouweler ${ }^{1}$, Natasja G. de Groot ${ }^{1}$ and Ronald E. Bontrop ${ }^{1,2, *}$

${ }^{1}$ Department of Comparative Genetics and Refinement, Biomedical Primate Research Centre, 2288 GJ Rijswijk, the Netherlands

${ }^{2}$ Theoretical Biology and Bioinformatics Group, Utrecht University, 3527 Utrecht, the Netherlands

*Corresponding author:

Ronald Bontrop

Bontrop@bprc.nl

Tel. nr. +31-15-2842699

Fax nr. +31-15-2842600 


\begin{abstract}
The killer cell immunoglobulin-like receptors (KIR) modulate immune responses through interactions with $\mathrm{MHC}$ class I molecules. The KIR region in large cohorts of rhesus and cynomolgus macaque populations were characterized, and the experimental design enabled the definition of a considerable number of alleles ( $n=576$ ) and haplotypes, which are highly variable with regard to architecture. Although high levels of polymorphism were recorded, only a few alleles are shared between species and populations. The rapid evolution of allelic polymorphism, accumulated by point mutations, was further confirmed by the emergence of a novel KIR allele in a rhesus macaque family. In addition to allelic variation, abundant orthologous and speciesspecific KIR genes were identified, the latter of which are frequently generated by fusion events. The concerted action of both genetic mechanisms, in combination with differential selective pressures at the population level, resulted in the unparalleled rapid evolution of the KIR gene region in two closely related macaque species. The variation of the $K I R$ gene repertoire at the species and population level might have an impact on the outcome of preclinical studies with macaque models.
\end{abstract}

\title{
Introduction
}

Natural killer (NK) cells provide an early defense mechanism against infectious diseases and tumor formation by their ability to recognize and kill cells with aberrant MHC class I expression (1-3). This immune surveillance is modulated by killer cell immunoglobulin-like receptors (KIR), which are expressed on NK cells and subsets of T cells (4-6). These gene products are transmembrane receptors consisting of two or three extracellular domains, which can facilitate ligand interaction, and a long or short cytoplasmic tail that can utilize an intracellular immunoreceptor tyrosine-based inhibitory or activation motif (ITIM or ITAM), respectively $(3,7)$. In humans, the gene family encoding the KIR genes is located on chromosome 19 q13.4, and its complexity is reflected by allelic polymorphism, gene copy number variation (CNV), chromosomal recombination, and alternative splicing (8-11).

Comparison of the KIR gene cluster in humans and other primate species suggests a first round of expansion between 30 and 45 million years ago (12), which involved two progenitor genes. The KIR3DX1 lineage is nowadays represented by a single copy in primates but expanded in cattle, whereas the KIR3D progenitor gene was subjected to diversification by tandem duplications, deletions, and recombinations $(13,14)$. This expansion resulted in a head-to-tail gene cluster encoding a broad repertoire of KIR genes, the overall architecture of which is conserved in primates. Species-specific diversification, however, may have resulted in differential lineage expansions and sequence variation, which is reflected by few KIR orthologs that are shared between distantly related primate species. Primate $K I R$ genes are phylogenetically classified into 
lineages based on receptor structure and ligand specificity. In humans, lineage I includes $K I R 2 D L 4$ and $K I R 2 D L 5$, lineage II $K I R 3 D L 1 / L 2 / S 1$, the expanded lineage III KIR2DL1-3, KIR2DS1-5, and the pseudogenes, and lineage $V$ KIR3DL3, respectively. The initial expansion of lineage III members can be traced back to orangutans, and its emergence seems to have co-evolved with the presence of HLA-C-like genes, which are present on approximately $50 \%$ of the contemporary orangutan $\mathrm{MHC}$ haplotypes (15). In chimpanzees and humans, the lineage III KIR genes expanded further, and their genomic clusters comprise 17 and 13 KIR genes, respectively, but only four genes are considered orthologs (14). Old World Monkeys (OWM), like macaques (genus macaca), expanded mainly lineage II KIR genes (KIR3D), which may be associated with their expanded MHC class I repertoire $(16,17)$.

Macaques are geographically the most widespread non-human primates (NHP) that diversified from the human and great ape lineage about 25 million years ago, and include approximately 20 species that share a habitat spanning from northeast Africa to Asia. Rhesus and cynomolgus macaques (Macaca mulatta, Macaca fascicularis) are closely related species that diverged from each other approximately 1-3 million years ago. Rhesus macaques are distributed across south, east, and Southeast Asia, whereas cynomolgus macaques mainly inhabit the mainland and islands of Southeast Asia. Geographically distinct populations, such as the Indian, Burmese and Chinese rhesus macaques, and the insular cynomolgus macaques, emerged by means of natural barriers, and resulted in intraspecific variation. The Isthmus of Kra, which is the narrowest part of the Malaysian peninsula, separates the cynomolgus macaques that inhabit the mainland of Southeast Asia in a northern (Cambodia, Thailand, and Vietnam) and southern (Malaysian peninsula) population, and it is suggested that this geographical barrier restricts gene flow $(18,19)$. In Indochina, rhesus and cynomolgus macaques may come across each other, and bidirectional introgression is substantiated by shared genetic features (20-22). For example, ancestral haplotypes of the highly polymorphic $\mathrm{MHC}$ class I region are encountered in rhesus macaques and cynomolgus macaques $(16,23,24)$, whereas extensive allele sharing had been documented for the MHC class II genes (25).

Several sequencing platforms have been used to characterize the macaque KIR gene region, particularly in Indian rhesus macaques (10, 26-30). Data on the KIR gene cluster and repertoire in other rhesus macaque populations is limited. For cynomolgus macaques, only the Mauritian animals were characterized thoroughly (31-33). This population was founded by a few animals that were introduced to the island by human interference, approximately 500 years ago, and therefore have a restricted KIR gene content.

Rhesus and cynomolgus macaques are used as preclinical models for many infectious and autoimmune diseases, as the immune response and pathologies reflect the human situation $(34,35)$. The origin of macaques, however, vary between different research facilities and might impact the disease phenotype, which is, for example, reported for SIV/AIDS studies in Indian and Chinese rhesus macaques $(36,37)$. The 
presence or absence of certain KIR genes, in combination with the MHC class I ligands, have been associated with disease susceptibility in both humans (6) and macaques $(38,39)$. A comprehensive overview of the KIR gene content and repertoire of different natural macaque populations is, however, lacking, despite the potential refinement for macaque models. Therefore, we set out to analyze the KIR transcriptomes of cohorts of rhesus and cynomolgus macaques of different geographical origins, which probably experienced varying selective pressures. Our observations illustrate in both highly related macaque species and populations an unparalleled form of rapid evolution of $K I R$ genes that is propelled by point mutations and complex chromosomal recombinations, which generate novel gene entities and result in highly variable haplotype architectures.

\section{Methods}

\section{Samples and origin}

Forty-six rhesus macaques, comprising 7 families, and 70 cynomolgus macaques, comprising 11 families, were selected from the self-sustaining colony housed the Biomedical Primate Research Centre. During the annual health checks, EDTA or heparin whole blood samples were obtained, and PBMCs were isolated from the latter. PBMC samples from 16 Chinese rhesus macaques, comprising 7 families, were obtained from the BPRC Bio-bank.

The geographical origin of most rhesus macaques was known based on importation records, such as the families from the Indian, Chinese, and Burmese populations. Additional transcriptome data of Indian rhesus macaques was incorporated from a previous KIR study conducted by our lab (10). The geographical origin of the cynomolgus macaques was mainly deduced by phylogenetic comparison of mitochondrial $12 \mathrm{~S}$ rRNA gene segments (40). With regard to this data, we defined three cynomolgus macaque populations, which originated from the mainland of Malaysia, from the Indonesian and Malaysian islands, and from Mauritius. The mainland population was further divided into populations north and south of the Isthmus of Kra. The origin of three cynomolgus macaques (Ji0603077, J15028, J16019) could not be determined unambiguously. In addition, previously reported KIR haplotypes of Mauritian cynomolgus macaques were added to the analysis (33).

\section{RNA isolation and KIR transcriptome amplification}

Total RNA was extracted directly from EDTA whole blood samples or from $\pm 15 \times 10^{6}$ PBMCs with RNeasy Mini Kit (Qiagen, Valencia, CA) in accordance with the manufacturer's instructions. First-strand cDNA was synthesized with the RevertAid First Strand cDNA Synthesis Kit (Invitrogen, Carlsbad, CA) using oligo(dT)18 primers. Genomic DNA was extracted from EDTA whole blood samples by a standard salting-out procedure, or from $\pm 15 \times 10^{6}$ PBMCs with an AllPrep RNA/DNA Mini Kit (Qiagen) according to the manufacturer's instructions. 
Full-length KIR transcripts were obtained by amplification of total cDNA with a KIR2DL04-specific and two KIR1D/KIR3D-generic primer sets, in accordance with a previously reported protocol (10). These primer sets were cross-reactive for the different rhesus and cynomolgus macaque populations. PCR products were sizeselected by gel-electrophoresis ( $\pm 1250 \mathrm{bp}$ ) and purified using a GeneJet Gel extraction kit (Invitrogen). The samples were pooled and purified twice using AMPure XP beads (Beckman Coulter, Woerden, the Netherlands) at a 1:1 bead to DNA volume ratio. The DNA concentration of purified pooled samples ( $>1 \mu \mathrm{g}$ total DNA) was measured using the Qubit dsDNA HS assay kit and Qubit 2.0 Fluorometer (Thermo Fisher Scientific).

PacBio SMRTbell libraries were generated according to Pacific Biosystems "Procedure \& Checklist - Amplicon Template Preparation and Sequencing", and sequencing was performed using a PacBio Sequel platform with a $10 \mathrm{~h}$ movie time using sequencing kit versions 2.0, 2.1, and 3.0, which was performed by the Leiden Genome Technology Center.

\section{PacBio data analysis}

Circular consensus sequences were selected for high read quality (value of 0.99 or higher), and demultiplexed based on unique barcoding.

Geneious Prime 2019 software was used to map the CCS reads to a database, consisting of reported rhesus macaque and novel cynomolgus macaque KIR sequences, to identify $100 \%$ matching reads ( $100 \%$ overlap, $0 \%$ mismatch, maximum ambiguity $=$ 1). The unused reads of related animals were grouped, and were de novo assembled. The consensus of each de novo contig was trimmed for the primer sequence, and phylogenetically aligned with the rhesus and cynomolgus macaque database. De novo sequences were confirmed when shown to segregate or when identified in two separate PCRs, and were subsequently submitted to the European Nucleotide Archive and assigned an accession number (https://www.ebi.ac.uk/ena/).

\section{Macaque KIR nomenclature}

The nomenclature of the KIR transcripts in rhesus and cynomolgus macaques follows the general guidelines of the KIR nomenclature report for non-human primates (NHP) (41). In brief, the name of the gene indicates the number of domains (1D, 2D, or 3D) and the signaling function ( $\mathrm{S}$ or $\mathrm{L}$ ). The inclusion of a "W" implies a workshop gene, which indicates a gene that is divergent on the basis of phylogenetic analysis but lacks sufficient reliability due to low frequency or to the absence of genomic sequencing or family studies. The inclusion of " $Q$ " indicates that it is questionable whether the transcripts are feasible. Two digits distinguish the different genes, and an asterisk followed by three digits distinguishes alleles. Two additional digits indicate synonymous variation.

Novel cynomolgus macaque KIR sequences were compared with a database of 342 reported rhesus macaque sequences $(10,26,29,30)$ and newly identified transcripts by phylogenetic analysis, using the Neighbor-Joining Tree building method (best tree mode) in MacVector software (MacVector, Cambridge, U.K.). Phylogenetic clusters were confirmed by the Maximum Likelihood Comparison and Neighbor-Joining Tree 
building methods in MEGA7 software, and all methods provided similar trees. Rhesus and cynomolgus macaque KIR sequences that clustered together with a close phylogenetic distance were considered orthologs, and received matching KIR gene names. Clusters of Mafa-KIR sequences that diverged from the other sequences according to sequence comparison and phylogenetic analysis received a workshop number. In addition, workshop numbers were assigned to cynomolgus macaque KIR genes that were thought to be the result of recombination events, as these are considered novel entities. In contrast, recombinant KIR genes in rhesus macaques are named after an allele of the gene that contributes the largest gene segment, as is described for this species in the NHP nomenclature report (41). The previously reported 46 Mafa-KIR sequences (33), all of which originated from the Mauritian cynomolgus macaque population, were also named.

\section{Macaque KIR haplotype origin and gene frequencies}

The origin of each KIR haplotype was categorized per macaque population. The populational origin of the rhesus macaques determined the haplotype origin, as none of the rhesus macaques had parents from different populations. The KIR haplotypes defined in cynomolgus macaques that had their roots in the mainland of Malaysia (north or south), or in the Malaysian/Indonesian islands, or in Mauritius were categorized based on the defined origin. In cynomolgus macaques with mixed roots (parents from the mainland and from islands), the origin of the KIR haplotypes was determined by the sequencing of parental genomic DNA, the origin of which was known, using a Mafa-KIR3DL2O exon 4-specific primer set (forward: 5'-GAAGAGACGGTCATCCTGCAGT-3'; reverse: 5'-ACTCCCCСTATGTGTTGTCAGC-3') and a Mafa-KIR1D exon 4-specific primer set (forward: 5'-GAAGAGACGGTCATCCTGCAGT-3'; reverse: 5'-АСТСССССТАTGTGTTGTCAGC-3'). Thermal cycling conditions were denaturation at $98^{\circ} \mathrm{C}$ for $2 \mathrm{~min}$, followed by 32 cycles of $98^{\circ} \mathrm{C}$ for $20 \mathrm{~s}, 63^{\circ} \mathrm{C}$ for $25 \mathrm{~s}$, and $72{ }^{\circ} \mathrm{C}$ for $1 \mathrm{~min}$. Amplicons of approximately $180 \mathrm{bp}$ were size-selected by gel electrophoresis and purified using a GeneJet Gel extraction kit (Invitrogen). Sanger sequencing was used, and the populational haplotype origin could be determined on the basis of three single nucleotide polymorphisms.

The frequency of a KIR gene in rhesus and cynomolgus macaques, or in one of the populations, was determined based on the presence of at least a single copy on a haplotype, the origin of which was determined, rather than on the presence of the gene in an individual. 


\section{Results}

\section{Definition of rhesus and cynomolgus macaque populations and their KIR transcriptomes}

The KIR transcriptomes of 62 rhesus and 70 cynomolgus macaques covering different populations were subjected to analysis (Figure 1) (10). All macaque samples belong to families that comprised two or more individuals, which allowed us to confirm the segregation of alleles but also to define haplotypes (Figure $\mathbf{2}$ and $\mathbf{3}$ ). The origin of the rhesus macaques was documented thoroughly, and included Burmese $(n=14)$, Chinese $(n=16)$, and Indian ( $n=32$ ) origins (Figure 1 ). Based on the phylogeny of

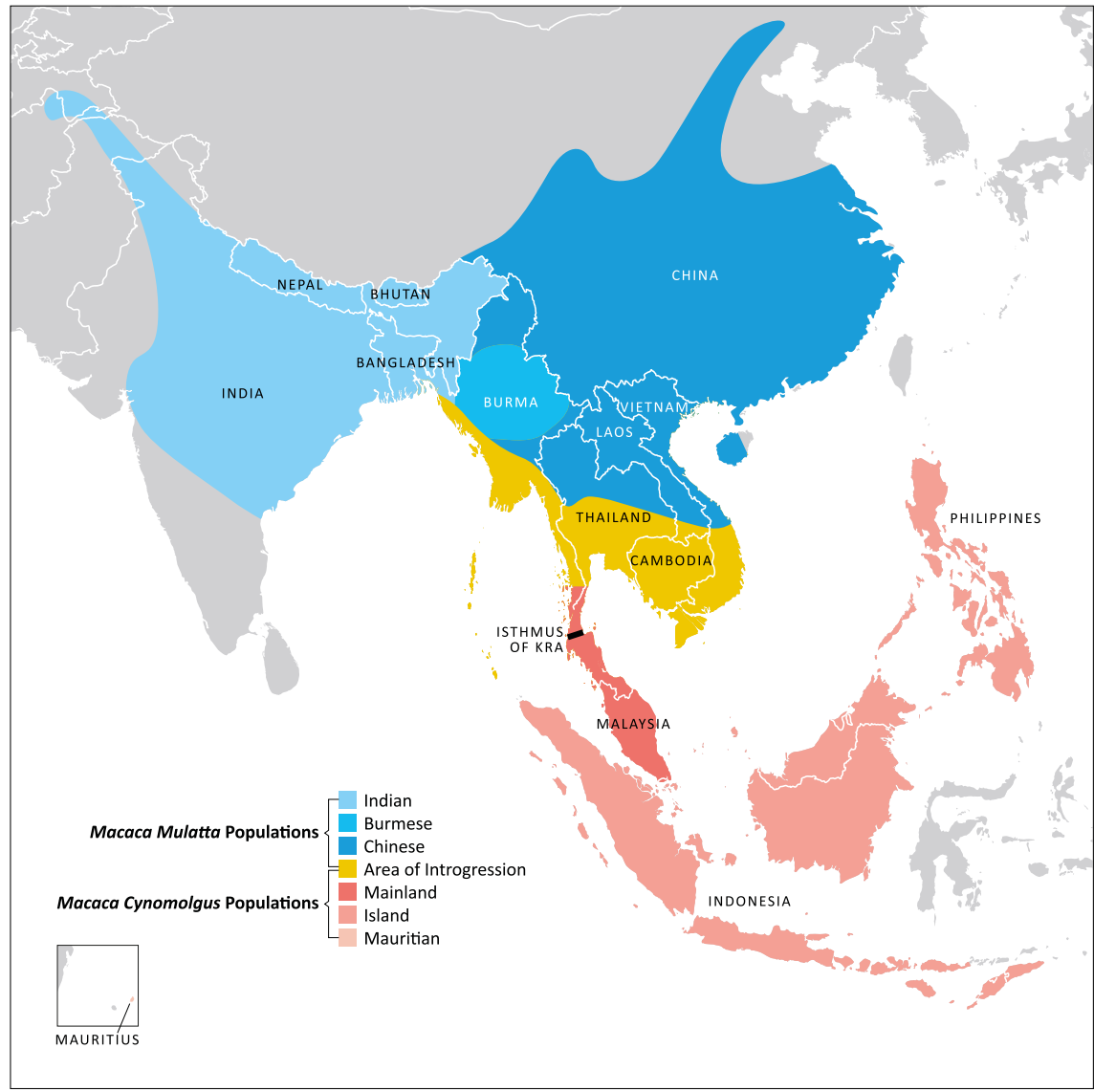

Figure 1. A schematic overview of the habitats of different rhesus and cynomolgus macaque populations. Three rhesus macaque populations (Indian, Burmese, and Chinese) are indicated by different blue colors, whereas four cynomolgus macaque populations (mainland: north and south of the Isthmus of Kra, Malaysian/ Indonesian islands, and Mauritius) are highlighted by red colors. The rhesus and cynomolgus macaque habitats include a hybrid zone (illustrated in yellow), in which introgression between the two species occurs. Mauritius is located approximately 4030 miles out of the South-east African coast, and is illustrated in a separate box. 
mtDNA sequences (40), origins of the cynomolgus macaques were mapped to the mainland of Southeast Asia $(n=26)$, the Malaysian/Indonesian islands ( $n=4)$, or Mauritius. The mainland population could be further divided into populations north ( $\mathrm{n}$ $=23$ ) and south $(n=3)$ of the Isthmus of Kra (Figure 1). For 19 cynomolgus macaques, a mixed origin was documented, whereas for 21 animals only the origin of a single parent could be determined. To expand our population panel, we included previously reported KIR transcriptome data from 30 Indian rhesus macaques (10) and 30 Mauritian cynomolgus macaques (33). Altogether, three rhesus and four cynomolgus macaque populations were subjected to comparison for their KIR repertoire.

\section{Allele discovery: abundant levels of species-specific allelic variation in macaques}

Up to now, 342 distinct rhesus macaque KIR alleles that were mainly isolated from Indian animals have been identified $(10,26,29,30)$. In the current cohort that comprises 32 Indian rhesus macaques, again another 48 unreported KIR alleles were discovered, indicating extensive allelic variation within this population. All Indian rhesus macaque $K I R$ alleles could be clustered into 22 different $K I R$ genes (Table I). From the Burmese and Chinese cohorts, 73 and 117 novel KIR alleles were isolated, respectively, which clustered to previously reported but also newly discovered KIR gene entities (Table I). During the course of this study, 34 rhesus macaque KIR genes were defined, which comprised 238 novel alleles, and 64 reported Mamu-KIR alleles were confirmed (Supp. Figure 1). The emergence of one of the novel alleles was observed in rhesus macaque R04104, which is expected to be homozygous for the KIR region, as it ought to receive two copies of Mamu-KIR3DLO5*006:01 via the H21-A haplotype (Figure 2 and 3). However, one copy of the Mamu-KIR3DL05*006:01 allele shows nonsynonymous mutations at two positions in the $\mathrm{D} 1$ domain $(T>C$ and $G>T$ ), thereby generating a novel allele, designated Mamu-KIR3DLO5*032. This de novo allele segregated with its corresponding haplotype (H21-B) into two offspring of R04014, and its existence was further substantiated by independent Sanger sequencing (Supp. Figure 2).

Most allelic variation is controlled by Mamu-KIR3DLO7, -KIR3DL20, and -KIR3DLO1, and to a lesser extent by -KIR2DLO4. The Indian and Burmese populations share four $K I R$ alleles, whereas only a single allele was shared between the Indian and Chinese (Mamu-KIR3DS06*016), the Burmese and Chinese populations (MamuKIR3DL05*007:01), and all three populations (Mamu-KIR3DL01*019:03) (Figure 4).

Knowledge of the KIR cluster in cynomolgus macaques is mainly confined to the artificially introduced Mauritian population, and 49 alleles are documented $(31,33)$. In the current cohort from different populations, we identified 267 novel alleles that clustered into 55 distinct KIR genes (Table II, Supp. Figure 1). In addition, 10 of the 46 previously reported Mafa-KIR sequences identified in Mauritian cynomolgus macaques were confirmed $(31,33)$. The highest level of allelic variation was observed for MafaKIR3DL2O and -KIR1D, followed by -KIR2DLO4, -KIR3DLO1, and -KIR3DLO7. The different populations seem to have highly unique allelic KIR repertoires. A single allele was shared between the northern mainland and the Indonesian/Malaysian populations 
Indian Rhesus Macaques
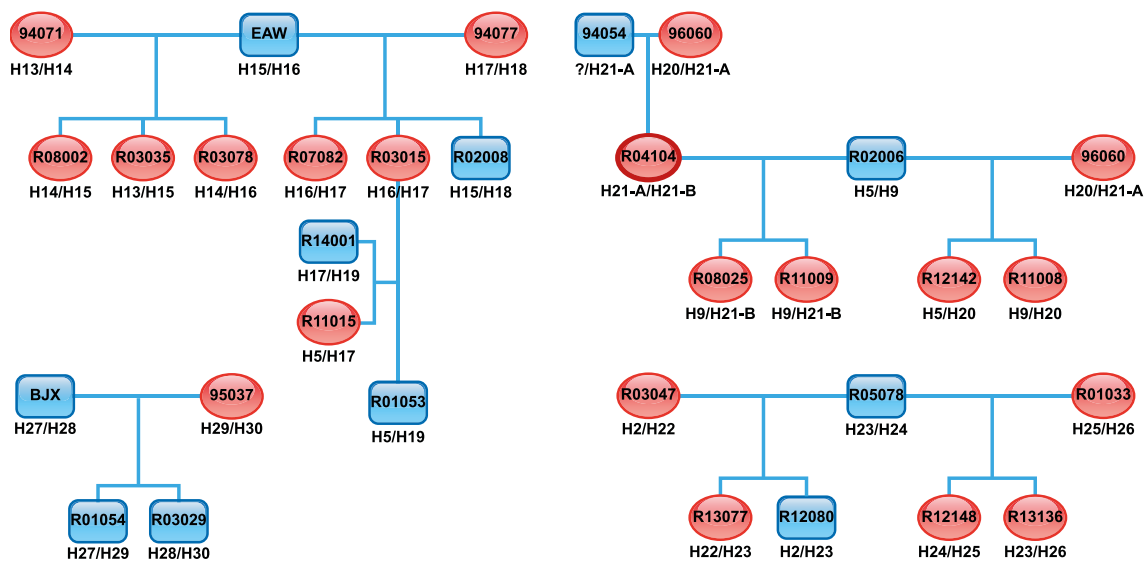

Burmese Rhesus Macaques
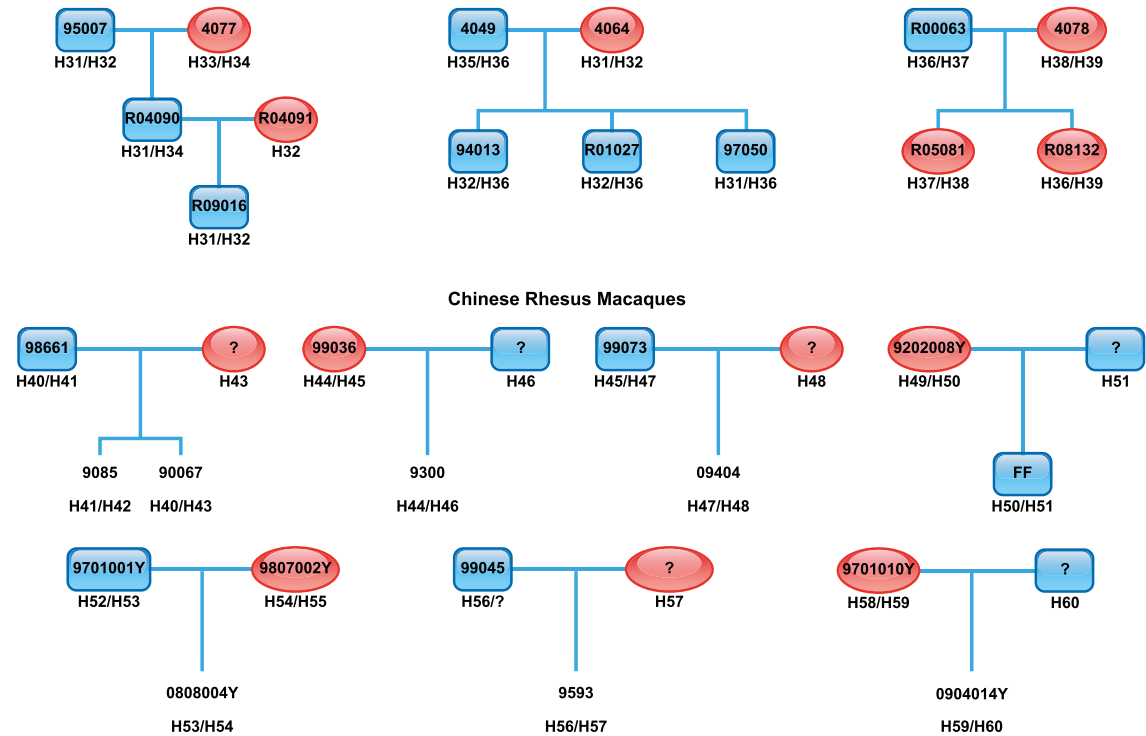

Figure 2. Rhesus macaque pedigrees. Fourteen rhesus macaque families are depicted and categorized by origin. Sires are indicated by blue squares, and dames by red ovals. For some offspring, the sex could not be determined. In six Chinese families, PBMC samples could be obtained from only a single parent, whereas the other parent is indicated with a question mark. Haplotype numbers are given for each animal and correspond to Figure 5. 

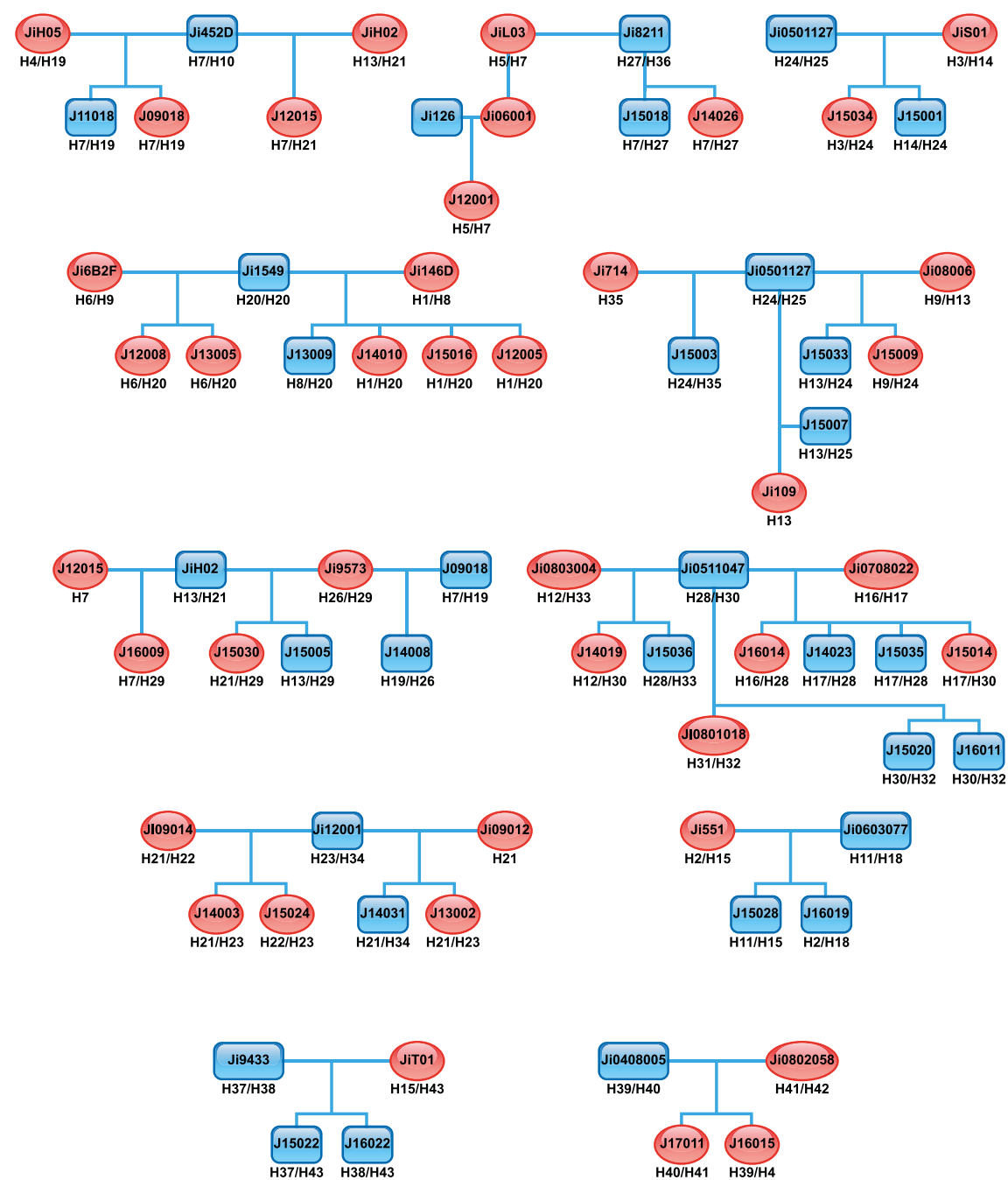

Figure 3. Cynomolgus macaque pedigrees. Eleven cynomolgus macaque families are depicted. Sires are indicated by blue squares, and dames by red ovals. Cynomolgus macaques from different populations are mixed in the families. Haplotype numbers are given for each animal and correspond to Figure 6.

(Mafa-KIR3DLW23*001), and the southern mainland and Mauritian populations (Mafa-KIR2DLO4*002), whereas two alleles were in common between the Indonesian/ Malaysian islands and Mauritian populations (Mafa-KIR3DLW13*003, MafaKIR3DLW26*001), and the southern mainland, the Indonesian/Malaysian islands, and Mauritian populations (Mafa-KIR1D*030Q, Mafa-KIR3DL20*002) (Figure 4). 
Table I. Overview of known and novel KIR alleles in rhesus macaques, indicated per $K I R$ gene and population. Known alleles were mainly reported in the Indian population [10, 25, 28, 29].

\begin{tabular}{|c|c|c|c|c|c|c|}
\hline \multirow[t]{2}{*}{ Gene } & \multirow[t]{2}{*}{ Known alleles } & \multicolumn{4}{|c|}{ Novel alleles } & \multirow[t]{2}{*}{ Total } \\
\hline & & Indian & Burmese & Chinese & Total novel & \\
\hline KIR1D & 5 & 2 & 3 & 3 & 8 & 13 \\
\hline KIR2DLO4 & 36 & 0 & 3 & 10 & 13 & 49 \\
\hline KIR3DL20 & 30 & 10 & 8 & 15 & 33 & 63 \\
\hline KIR3DLO1 & 42 & 3 & 7 & 13 & 23 & 65 \\
\hline KIR3DLO2 & 15 & 0 & 2 & 4 & 6 & 21 \\
\hline KIR3DLW03 & 9 & 1 & 3 & 13 & 17 & 26 \\
\hline KIR3DLO4 & 4 & 0 & 0 & 0 & 0 & 4 \\
\hline KIR3DLO5 & 28 & 3 & 3 & 5 & 11 & 39 \\
\hline KIR3DLO6 & 2 & 0 & 4 & 2 & 6 & 8 \\
\hline KIR3DLO7 & 34 & 11 & 11 & 17 & 39 & 73 \\
\hline KIR3DLO8 & 19 & 5 & 3 & 4 & 12 & 31 \\
\hline KIR3DL10 & 10 & 1 & 1 & 1 & 3 & 13 \\
\hline KIR3DL11 & 13 & 1 & 0 & 5 & 6 & 19 \\
\hline KIR3DLW12 & 0 & 0 & 0 & 2 & 2 & 2 \\
\hline KIR3DLW14 & 0 & 1 & 1 & 2 & 4 & 4 \\
\hline KIR3DLW17 & 0 & 0 & 1 & 2 & 3 & 3 \\
\hline KIR3DLW18 & 0 & 0 & 1 & 0 & 1 & 1 \\
\hline KIR3DLW25 & 0 & 0 & 1 & 0 & 1 & 1 \\
\hline KIR3DS01 & 8 & 1 & 0 & 1 & 2 & 10 \\
\hline KIR3DSO2 & 21 & 2 & 8 & 4 & 14 & 35 \\
\hline KIR3DSO3 & 5 & 1 & 0 & 0 & 1 & 6 \\
\hline KIR3DSO4 & 11 & 1 & 2 & 2 & 5 & 16 \\
\hline KIR3DSO5 & 10 & 0 & 1 & 0 & 1 & 11 \\
\hline KIR3DSO6 & 14 & 2 & 1 & 4 & 7 & 21 \\
\hline KIR3DSW07 & 4 & 0 & 0 & 1 & 1 & 5 \\
\hline KIR3DSW08 & 13 & 2 & 2 & 3 & 7 & 20 \\
\hline KIR3DSW09 & 9 & 1 & 0 & 1 & 2 & 11 \\
\hline KIR3DSW10 & 0 & 0 & 1 & 0 & 1 & 1 \\
\hline KIR3DSW16 & 0 & 0 & 1 & 0 & 1 & 1 \\
\hline KIR3DSW18 & 0 & 0 & 1 & 0 & 1 & 1 \\
\hline KIR3DSW2O & 0 & 0 & 1 & 0 & 1 & 1 \\
\hline KIR3DSW21 & 0 & 0 & 2 & 2 & 4 & 4 \\
\hline KIR3DSW32 & 0 & 0 & 0 & 1 & 1 & 1 \\
\hline KIR3DSW34 & 0 & 0 & 1 & 0 & 1 & 1 \\
\hline Total & 342 & 48 & 73 & 117 & 238 & 580 \\
\hline
\end{tabular}

To sum up, 579 KIR alleles were identified in the rhesus and cynomolgus macaque populations studied. Only two alleles were shared between both highly related species: namely, Mamu-KIR3DLW12*002/Mafa-KIR3DLW12*006 and Mamu- and MafaKIR3DLW18*001 (Figure 4). The low number of allele sharing between the macaque species as well as the different populations suggests fast evolution. This is within 


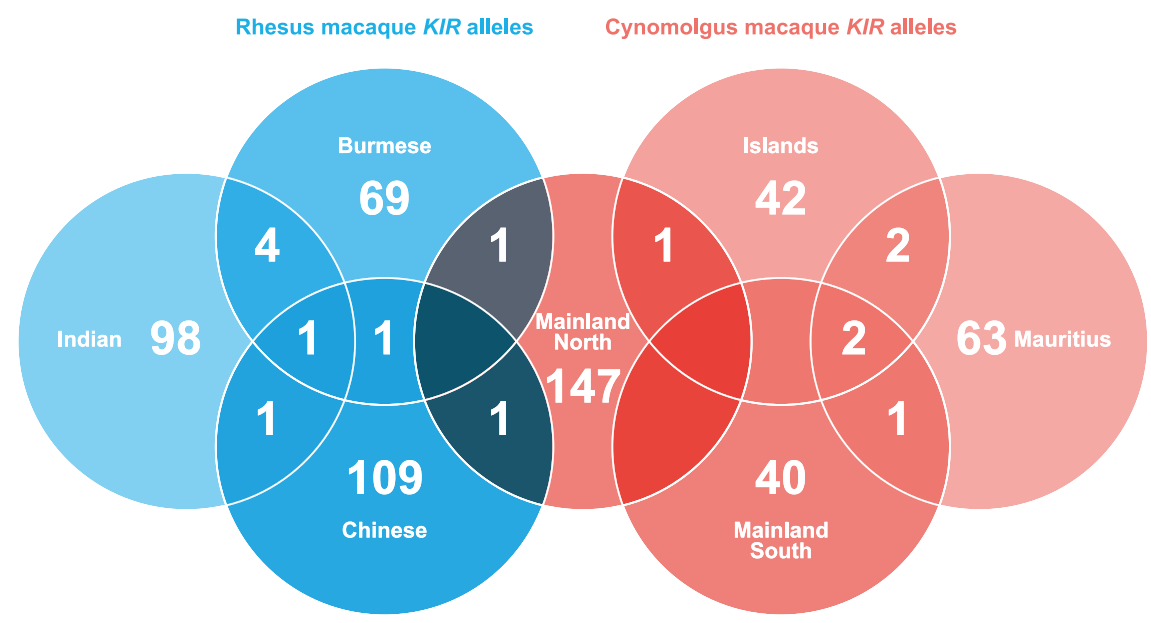

Figure 4. KIR allele distribution in rhesus and cynomolgus macaque populations. The three rhesus macaque populations are indicated in blue circles, and the four cynomolgus macaque populations are depicted in red circles. The total number of uniquely identified $K I R$ alleles is provided per population and the number of shared KIR alleles is indicated for the involved populations.

lineages mainly mediated by point mutations, and contrasts the extensive sharing documented for $\mathrm{MHC}$ class II and, to a much lesser extent, for MHC class I alleles (42).

\section{New KIR genes in macaques are generated by recombination}

Considering a shared ancestor living 1-3 million years ago, one might expect highly similar repertoires of orthologous KIR genes in rhesus and cynomolgus macaques, as is observed for the closely related Bornean and Sumatran orangutan species (15). Apparently, however, this is not the case in both macaque species, as their KIR gene repertoires possess species-specific and a differential number of $K I R$ gene moieties. Moreover, the 34 rhesus and 55 cynomolgus macaque KIR genes that are defined by sequence comparison and phylogenetic analysis indicate a greater expansion of the $K I R$ gene repertoire in macaques as compared to humans and other primate species, for which 17 or fewer KIR genes were identified $(41,43,44)$. The question to be answered therefore is how are new KIR genes generated. One mechanism that might explain the expanded macaque $K I R$ gene repertoire is the occurrence of abundant recombination events, which result in the formation of hybrid genes composed of segments from two different KIR genes (Tables III and IV). Along with others, we found evidence of similar events in humans $(10,45)$, although this mechanism seems to happen more frequently in macaques. In rhesus macaques, hybrid KIR genes are named after the allele that contribute the largest segment (41). For example, multiple entities have a large Mamu-KIR3DLO7 segment, which is found in conjunction with a smaller segment of -KIR3DL05,-KIR3DLO8, or -KIR3DSW08, but all are named and listed as alleles of Mamu-KIR3DLO7 (Table III). Another peculiar recombination event 
Table II. Overview of known and novel KIR alleles in cynomolgus macaques, indicated per KIR gene.

\begin{tabular}{|c|c|c|c|}
\hline Gene & $\begin{array}{l}\text { Known } \\
\text { alleles }\end{array}$ & $\begin{array}{l}\text { Novel } \\
\text { alleles }\end{array}$ & Total \\
\hline KIR1D & 4 & 30 & 34 \\
\hline KIR2DLO4 & 3 & 38 & 41 \\
\hline $3 D L 20$ & 1 & 33 & 34 \\
\hline 3DLO1 & 2 & 14 & 16 \\
\hline 3DLW03 & 0 & 4 & 4 \\
\hline 3DLO5 & 0 & 5 & 5 \\
\hline 3DLO6 & 0 & 1 & 1 \\
\hline 3DLO7 & 3 & 15 & 18 \\
\hline $3 D L 11$ & 2 & 16 & 18 \\
\hline $3 D L W 12$ & 3 & 11 & 14 \\
\hline $3 D L W 13$ & 3 & 6 & 9 \\
\hline 3DLW14 & 2 & 6 & 8 \\
\hline $3 D L W 15$ & 1 & 3 & 4 \\
\hline $3 D L W 16$ & 2 & 2 & 4 \\
\hline $3 D L W 17$ & 0 & 3 & 3 \\
\hline $3 D L W 18$ & 0 & 1 & 1 \\
\hline $3 D L W 19$ & 1 & 1 & 2 \\
\hline $3 D L W 21$ & 0 & 1 & 1 \\
\hline $3 D L W 22$ & 0 & 1 & 1 \\
\hline $3 D L W 23$ & 0 & 1 & 1 \\
\hline $3 D L W 24$ & 1 & 0 & 1 \\
\hline $3 D L W 25$ & 2 & 2 & 4 \\
\hline $3 D L W 26$ & 1 & 0 & 1 \\
\hline $3 D L W 27$ & 0 & 1 & 1 \\
\hline $3 D L W 28$ & 1 & 0 & 1 \\
\hline $3 D L W 29$ & 0 & 1 & 1 \\
\hline $3 D L W 30$ & 0 & 1 & 1 \\
\hline $3 D S 02$ & 0 & 2 & 2 \\
\hline
\end{tabular}

\begin{tabular}{|c|c|c|c|}
\hline Gene & $\begin{array}{c}\text { Known } \\
\text { alleles }\end{array}$ & $\begin{array}{l}\text { Novel } \\
\text { alleles }\end{array}$ & Total \\
\hline $3 D S 04$ & 0 & 1 & 1 \\
\hline $3 D S 06$ & 0 & 3 & 3 \\
\hline 3DSW07 & 0 & 3 & 3 \\
\hline 3DSW08 & 0 & 3 & 3 \\
\hline $3 D S W 10$ & 1 & 3 & 4 \\
\hline $3 D S W 11$ & 0 & 4 & 4 \\
\hline $3 D S W 12$ & 1 & 9 & 10 \\
\hline $3 D S W 13$ & 2 & 0 & 2 \\
\hline $3 D S W 14$ & 0 & 2 & 2 \\
\hline 3DSW15 & 0 & 9 & 9 \\
\hline $3 D S W 16$ & 0 & 3 & 3 \\
\hline 3DSW17 & 3 & 1 & 4 \\
\hline $3 D S W 18$ & 0 & 2 & 2 \\
\hline 3DSW19 & 0 & 2 & 2 \\
\hline 3DSW20 & 1 & 3 & 4 \\
\hline $3 D S W 21$ & 2 & 5 & 7 \\
\hline 3DSW22 & 1 & 2 & 3 \\
\hline $3 D S W 23$ & 0 & 1 & 1 \\
\hline 3DSW24 & 2 & 3 & 5 \\
\hline $3 D S W 25$ & 1 & 0 & 1 \\
\hline $3 D S W 26$ & 0 & 1 & 1 \\
\hline $3 D S W 27$ & 1 & 1 & 2 \\
\hline 3DSW28 & 1 & 0 & 1 \\
\hline 3DSW29 & 1 & 1 & 2 \\
\hline $3 D S W 30$ & 0 & 3 & 3 \\
\hline 3DSW31 & 0 & 2 & 2 \\
\hline 3DSW33 & 0 & 1 & 1 \\
\hline Total & 49 & 267 & 316 \\
\hline
\end{tabular}

resulted in Mamu-KIR3DSO4*011, the extracellular domains (exons 1-5) of which originate from -KIR3DSO4, whereas the cytoplasmic tail is similar to exons 6-9 of -KIR3DLO7. The name is therefore somewhat confusing as this gene is listed as an allele of Mamu-KIR3DSO4, although it encodes an inhibitory cytoplasmic tail. In rhesus macaques, at least 19 hybrid KIR genes were generated by recombination events (Table III). It would seem that for some of these hybrids the nomenclature is in need of attention (46). From a more general and functional perspective, hybrid gene entities could encode novel genes with potentially distinct functional features, due to differential combinations of ligand binding domains and signal transduction elements.

In cynomolgus macaques, at least seven hybrid KIR genes were detected (Tables II and IV). For example, the first 6 exons of Mafa-KIR3DSW21 are highly similar (98-99\%) to those of $-K I R 3 D L 07$, whereas the transmembrane region and cytoplasmic tail of 
Table III. Novel gene entities that are generated by chromosomal recombination events in rhesus macaques. The gene donors and corresponding donated segments are indicated. For some novel entities, only a single donor could be identified. The novel entities are named as an allele of the gene that contributed the largest segment.

\begin{tabular}{|c|c|c|c|c|}
\hline \multirow[b]{2}{*}{ Novel entity (allele name) } & \multicolumn{2}{|c|}{ Gene segment 1} & \multicolumn{2}{|c|}{ Gene segment 2} \\
\hline & Gene donor 1 & Segment & Gene donor 2 & Segment \\
\hline Mamu-KIR3DL01*054 & Mamu-KIR3DL05 & Exons 1- 3 & Mamu-KIR3DLO1 & Exons 4- 9 \\
\hline Mamu-KIR3DL02*005, *011 & Mamu-KIR3DLO2 & Exons $1-6$ & Mamu-KIR3DLO1 & Exons 7-9 \\
\hline Mamu-KIR3DL02*006, *010 & Mamu-KIR3DLO2 & Exons 1- 7 & Mamu-KIR3DLO1 & Exons 8-9 \\
\hline Mamu-KIR3DL02*016 & Mamu-KIR3DL10 & Exons 1- 4 & Mamu-KIR3DLO2 & Exons 5-9 \\
\hline Mamu-KIR3DLW03*023 & Unknown donor & Exons $1-3$ & Mamu-KIR3DLW03 & Exons 4- 9 \\
\hline Mamu-KIR3DL05*029, *030, *033 & Mamu-KIR3DL05 & Exons 1- 7 & Unknown donor & Exons $8-9$ \\
\hline Mamu-KIR3DL07*042 & Mamu-KIR3DLO7 & Exons 1- 5 & Mamu-KIR3DL08 & Exons $6-9$ \\
\hline Mamu-KIR3DL07*045 & Mamu-KIR3DLO5 & Exons 1- 3 & Mamu-KIR3DLO7 & Exons 4- 9 \\
\hline Mamu-KIR3DL07*056 & Mamu-KIR3DSW08 & Exons 1-3 & Mamu-KIR3DLO7 & Exons 4- 9 \\
\hline Mamu-KIR3DL07*064 & Mamu-KIR3DLO5 & Exons 1- 4 & Mamu-KIR3DLO7 & Exons $5-9$ \\
\hline Mamu-KIR3DL07*065 & Unknown donor & Exons $1-3$ & Mamu-KIR3DLO7 & Exons 4- 9 \\
\hline Mamu-KIR3DL08*018, *019, *020 & Mamu-KIR3DLO2 & Exons 1- 3 & Mamu-KIR3DL08 & Exons 4- 9 \\
\hline Mamu-KIR3DL08*021 & Mamu-KIR3DL01 & Exons 1- 4 & Mamu-KIR3DL08 & Exons $5-9$ \\
\hline Mamu-KIR3DL20*030 & Mamu-KIR3DL20 & Exons 1- 7 & Mamu-KIR2DLO4 & Exons 8- 9 \\
\hline Mamu-KIR3DL20*044 & Mamu-KIR3DL20 & Exons 1- 7 & Mamu-KIR1D & Exons 8- 9 \\
\hline Mamu-KIR3DSO2*012, *029 & Mamu-KIR3DSO2 & Exons 1- 4 & Mamu-KIR3DSW09 & Exons 5-9 \\
\hline Mamu-KIR3DS04*011 & Mamu-KIR3DSO4 & Exons 1- 5 & Mamu-KIR3DLO7 & Exons $6-9$ \\
\hline Mamu-KIR3DSO6*019 & Mamu-KIR3DSW07 & Exons 1- 3 & Mamu-KIR3DSO6 & Exons 4- 9 \\
\hline Mamu-KIR3DSW09*005, *011 & Mamu-KIR3DSW08 & Exons 1 - 3 & Unknown donor & Exons 4- 9 \\
\hline
\end{tabular}

-KIR3DSW21 is identical to -KIR3DSW12. This suggests that Mafa-KIR3DSW21 may interact with similar ligands as $-K I R 3 D L 07$, but that it transduces activating instead of inhibitory signals. Seven Mafa-KIR3DSW21 alleles are identified (Table II), suggesting positive selection for variation on the gene products generated by this recombination event.

The origin of both segments could not be identified for all hybrid KIR genes. MamuKIR3DL05*029/*030/*033, Mafa-KIR3DLW24, Mafa-KIR3DLW29, Mafa-KIR3DSW18, and Mafa-KIR3DSW2O seem to have segments of Mamu-KIR3DLO5, Mafa-KIR3DLW12, 
Table IV. Novel gene entities that are generated by chromosomal recombination events in cynomolgus macaques. The gene donors and corresponding donated segments are indicated. For some novel entities, only a single donor could be identified. The novel entities received a gene workshop number.

\begin{tabular}{lllll}
\hline & \multicolumn{2}{c}{ Gene segment 1 } & \multicolumn{2}{c}{ Gene segment 2 } \\
\hline Novel entity (gene name) & Gene donor 1 & Segment & Gene donor 2 & Segment \\
\hline Mafa-KIR3DLW24 & Unknown donor & Exons 1-3 & Mafa-KIR3DLW12 & Exons 4- 9 \\
\hline Mafa-KIR3DLW26 & Mafa-KIR3DSW15 & Exons 1- 3 & Mafa-KIR3DLW27*001 & Exons 4- 9 \\
\hline Mafa-KIR3DLW27 & Mafa-KIR3DSW22*001 & Exons 1-4 & Mafa-KIR3DLW26*001 & Exons 5-9 \\
\hline Mafa-KIR3DLW29 & Unknown donor & Exons 1-4 & Mafa-KIR3DLW13 & Exons 5- 9 \\
\hline Mafa-KIR3DSW18 & Mafa-KIR3DSW17 & Exons 1-4 & Unknown donor & Exons 5- 9 \\
\hline Mafa-KIR3DSW20 & Mafa-KIR3DSW19 & Exons 1-4 & Unknown donor & Exons 5- 9 \\
\hline Mafa-KIR3DSW21 & Mafa-KIR3DL07 & Exons 1- 6 & Mafa-KIR3DSW12 & Exons 7-9 \\
\hline
\end{tabular}

Mafa-KIR3DLW13, Mafa-KIR3DSW17, and Mafa-KIR3DSW19, respectively, but it was not possible to trace the donor of the other segment (Tables III and IV). This indicates that when more sequences become available, additional hybrid KIR gene entities and segments are likely to be defined in macaques.

Within the macaque KIR repertoire studied, 24 macaque $K I R$ genes are highly similar, and are considered to be orthologs. These genes most likely represent a single locus in both species, although it is too early to elucidate their exact physical location, as the relevant genomic studies are in progress. One would expect that the number of 24 orthologs shared between two closely related macaque species reflects common ancestry, whereas the relatively high number of species-specific KIR genes indicates the rapid generation of novel gene entities, which can, in part, be explained by abundant recombination.

\section{Definition of macaque KIR haplotypes}

The family-based study design resulted in the thorough characterization of 49 rhesus and 43 cynomolgus macaque KIR haplotypes (Figures 5 and 6), which are categorized on the basis of geographical origin of the analysed animals (Figure 1). The rhesus macaque KIR haplotypes are referred to as $\mathrm{Rh}-\mathrm{H} 13$ to $\mathrm{Rh}-\mathrm{H} 60$, consecutively to the 12 previously reported haplotypes (Figure 5) (10). Cynomolgus macaque KIR haplotypes are referred to as $\mathrm{Cy}-\mathrm{H} 1$ to $\mathrm{Cy}-\mathrm{H} 43$, whereas the previously reported Mauritian chromosomal KIR configurations are listed as K1 to K8 (Figure 6) (33). All these haplotypes display extensive copy number variation. The rhesus macaque haplotypes encoded 4 to 17 KIR transcripts, whereas the cynomolgus macaque equivalents encoded 3 to 13 KIR transcripts. KIR3DL20 was identified on most macaque haplotypes, except for haplotype $\mathrm{Cy}-\mathrm{H} 39$, and seems to be absent on the haplotypes of the Mauritian animals. However, we assume that KIR3DL2O should be considered a framework gene in macaques, and that a few transcripts were missed due to primer 

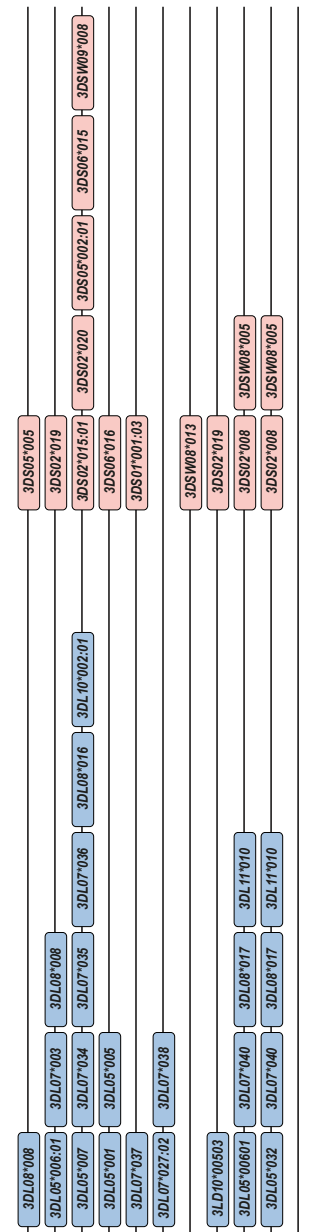

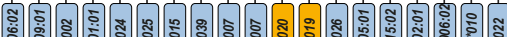
ถัํำ ติ๊

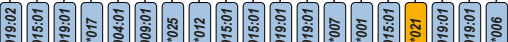

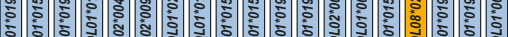

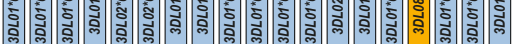

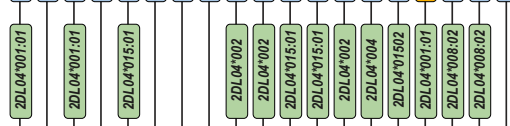

$\stackrel{g}{\Xi}$

兽

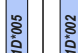

\section{ธิ์}

:

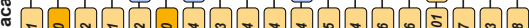

Е ₹

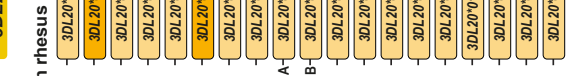

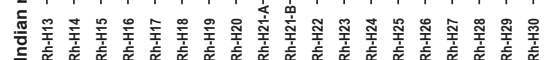

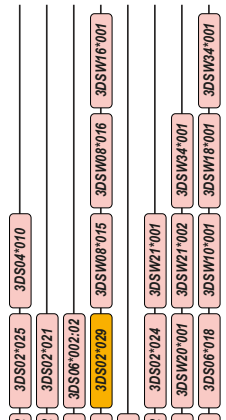

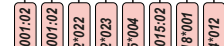

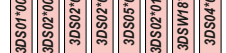

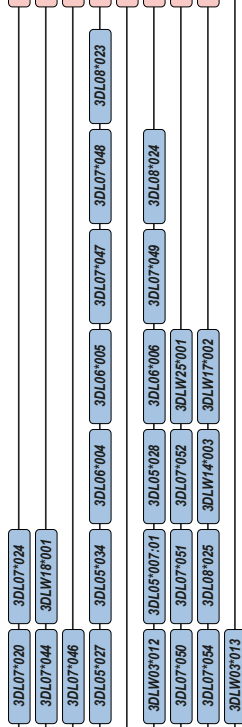

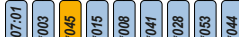

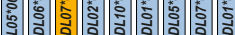

के ले क्ले क्ल क्ले क्ले

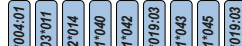

空

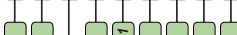

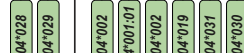

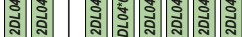

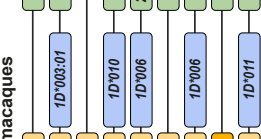

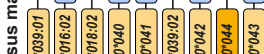

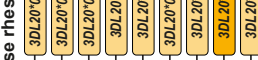

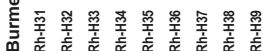




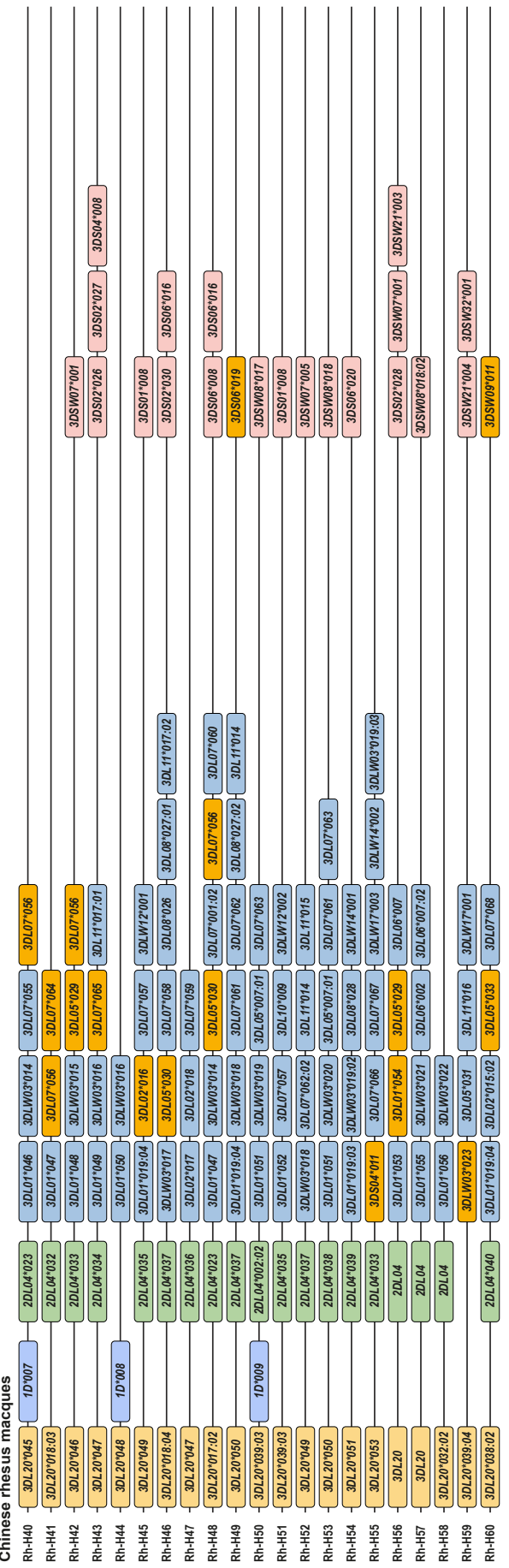

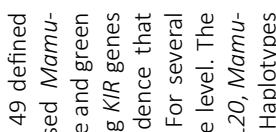

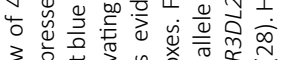

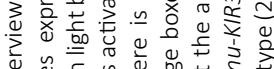

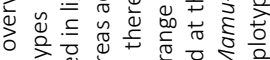

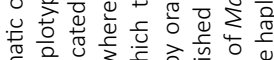

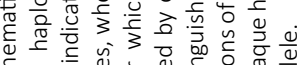

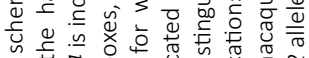

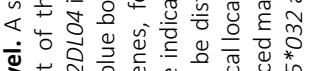

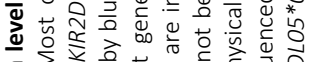

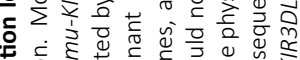

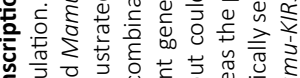

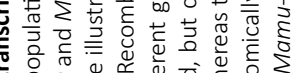

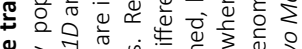

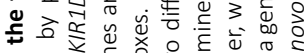

t.

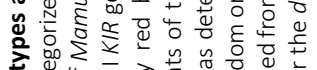

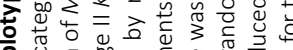

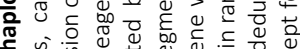

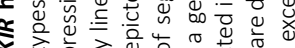

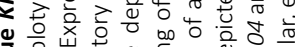

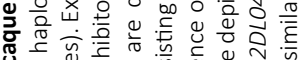

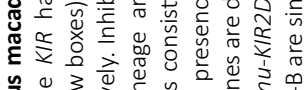

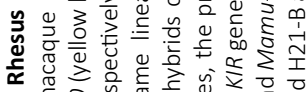

ㄷ.

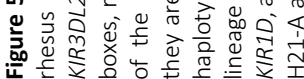



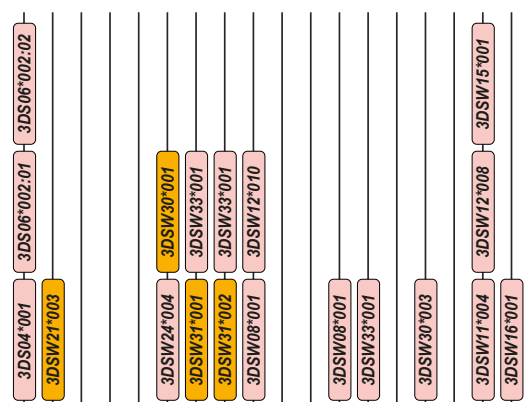

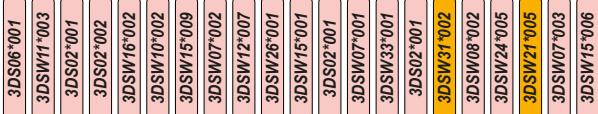
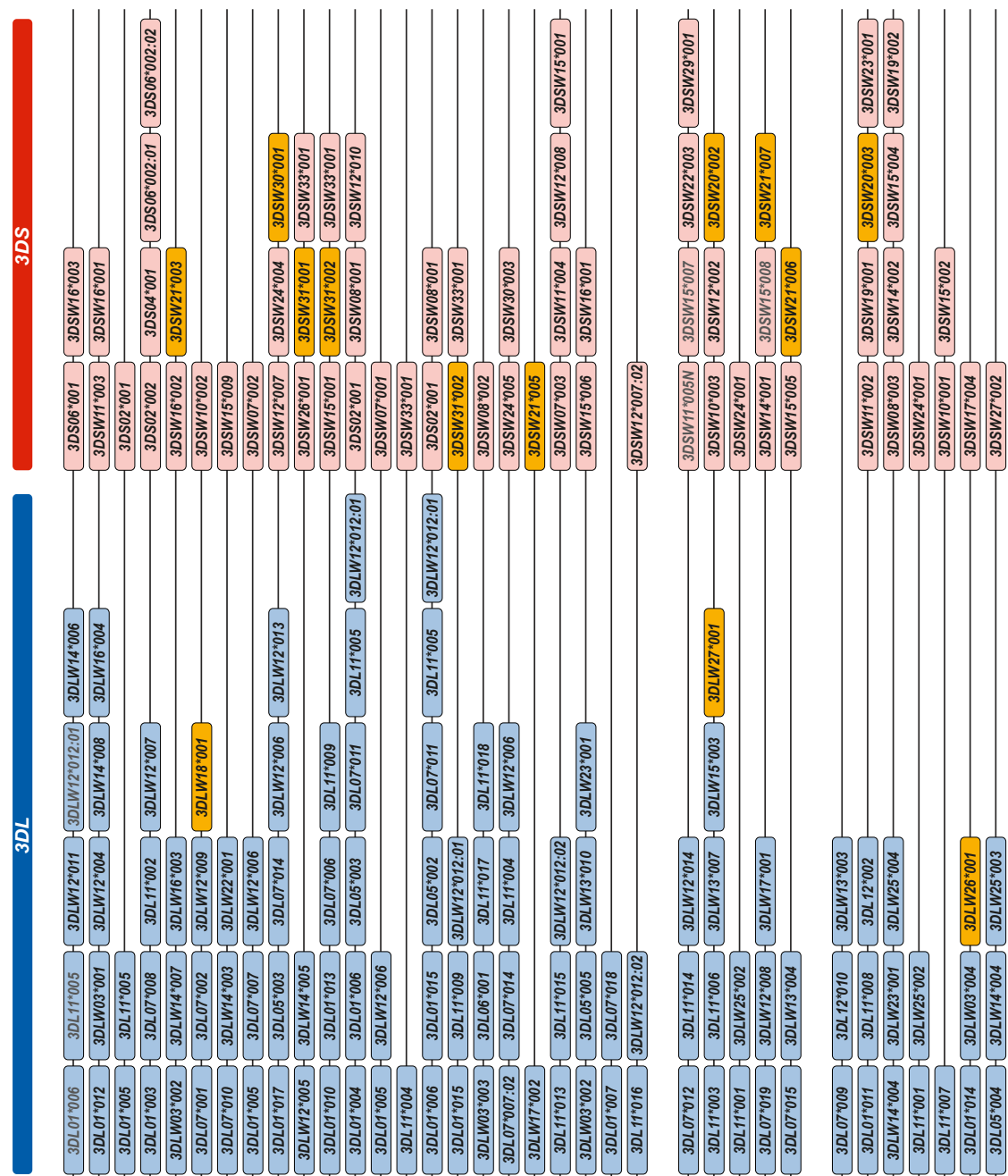

¿

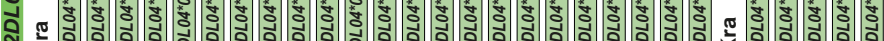

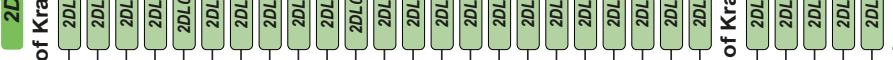

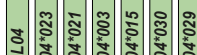

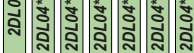

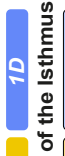
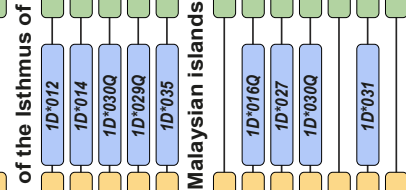

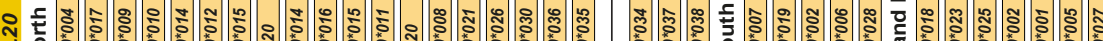

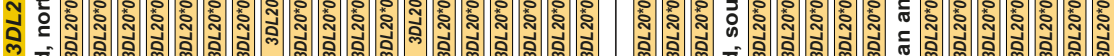

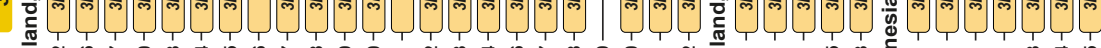

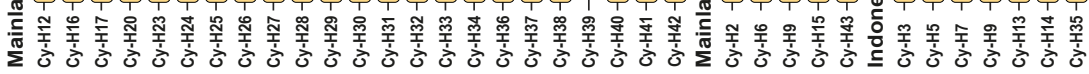




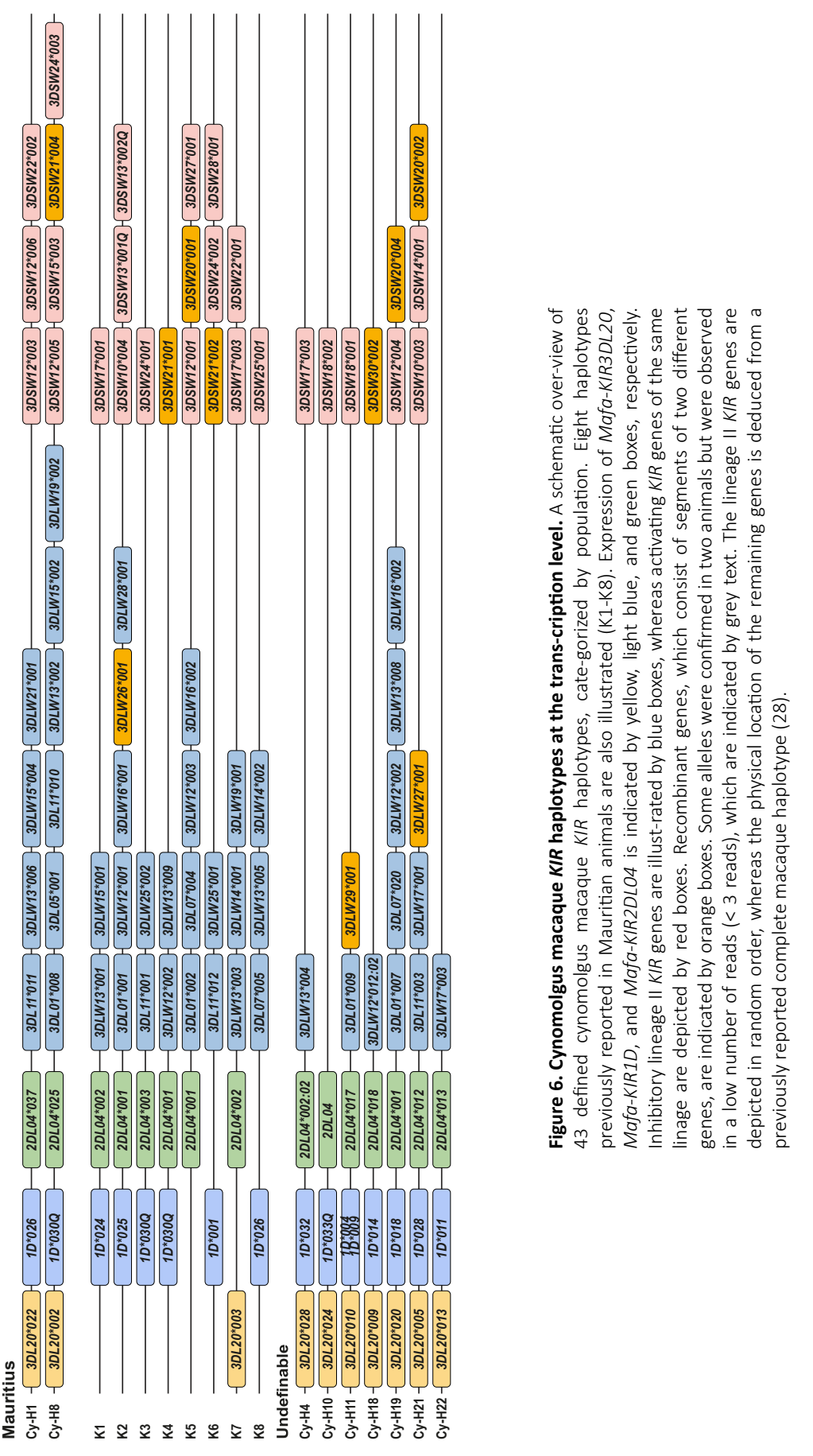


inconsistencies. For the Mauritian cynomolgus macaque, this assumption is confirmed by haplotype $\mathrm{Cy}-\mathrm{H} 9$, which is identical to $\mathrm{K} 3$, defined by another research team, except for the presence of Mafa-KIR3DL20*002. Haplotype Cy-H9/K3 is identified in three populations, and may indicate an ancestral origin. KIR2DLO4 is observed on $70 \%$ and $94 \%$ of the rhesus and cynomolgus macaque KIR haplotypes, respectively, and represent the only reported macaque KIR gene that shares an apparent ortholog with humans. In humans, this gene is considered a framework gene, and there is support that this might also be the case for its cynomolgus macaque ortholog (47).

\section{Recombination influences haplotype architecture and drives copy number variation}

Chromosomal recombinations such as unequal crossing over, gene fusion and gene duplications can expand or contract a KIR haplotype, thereby affecting the genetic content. Two or more apparent allelic copies of a given KIR gene were identified on 23 of the 49 rhesus and 11 of the 43 cynomolgus macaque KIR haplotypes. It is likely that such genes were once orthologs, but owing to complex recombination events they might end up as paralogs. These duplications involved mainly lineage II inhibitory KIR genes, such as KIR3DL01, KIR3DLO7, and KIR3DL11, but also Mamu-KIR3DL20 (RhH27), Mafa-KIR1D (Cy-H11), and Mafa-KIR3DSW12 (Cy-H1). In total, 15 different KIR genes are duplicated on the listed haplotypes, 11 of which are considered orthologs. This suggests that ancestral genes are more often subject to duplication as compared to more recently generated species-specific KIR genes. The most extensive copy number variation is witnessed for Mamu-KIR3DLO1 on haplotype Rh-H26 (Figure 5), on which four allelic copies exist.

Hybrid KIR genes (Tables III and IV) are associated with chromosomal recombination events, and might mark expanded and contracted KIR haplotypes. For example, two hybrid Mamu-KIR3DL20 genes, composed of exons encoding the extracellular domains of Mamu-KIR3DL2O (exons 1-7), and the cytoplasmic tail of Mamu-KIR1D (Rh-H38) or Mamu-KIR2DLO4 (Rh-H14,-H18), seem to coincide with a centromeric haplotype contraction. Also, haplotype Rh-H27 carries an example of a gene that consists of the first four exons of Mamu-KIR3DLO1 and the last five exons of Mamu-KIR3DLO8 (Table III, Figure 5). The formation of this gene probably resulted in another contracted haplotype, as only four KIR genes are present at the telomeric end. In the cynomolgus macaque, haplotypes $\mathrm{Cy}-\mathrm{H} 6$ and-H21 seem to be expanded, marked by the presence of the recombinant genes Mafa-KIR3DLW27 and Mafa-KIR3DSW20, whereas the relatively short haplotype $\mathrm{Cy}-\mathrm{H} 38$ contains another hybrid gene Mafa-KIR3DSW21, the emergence of which might have resulted in a contraction (Table IV, Figure 6).

This in-frame fusion mechanism occurs rather frequently, as 21 rhesus macaque and 21 cynomolgus macaque haplotypes contain a recombinant gene (Figure $\mathbf{5}$ and $\mathbf{6}$ ), although not each hybrid gene seems to mark contraction or expansion. Thus, the KIR gene cluster in both macaque species seems to be subjected to frequent gene duplications and chromosomal recombination events, which not only generate novel gene entities but also result in a differential KIR haplotype architecture. 


\section{KIR gene frequencies differ between species}

The occurrence of at least 24 orthologs in both macaque species is most likely due to the sharing of a common ancestor, but introgression between the two species may also have an impact. The frequency of these orthologs, however, differs considerably between both macaque species (Figure 7). The orthologous genes that are encountered more frequently on rhesus than on cynomolgus macaque haplotypes are KIR3DLO1, KIR3DLW03, KIR3DLO5, KIR3DLO6, KIR3DLO7, KIR3DSO2, KIR3DSO6, and KIR3DSW08. It is noted that for these genes the allelic variation is higher in rhesus macaques than in cynomolgus macaques (Tables I and II).

Other orthologs are more often present in cynomolgus macaques, such as KIR3DL11, KIR3DLW12, KIR3DLW14, KIR3DLW25, KIR3DSW10, KIR3DSW20, and KIR3DSW21, that, with the exception of KIR3DL11, display greater allelic variation compared to rhesus macaques (Tables I and II). An exceptional example is formed by Mafa-KIR1D, which is present on $82 \%$ of the haplotypes in cynomolgus monkeys but only on $22 \%$ of the haplotypes in rhesus macaques. Moreover, the allelic variation of Mafa-KIR1D exceeds that of Mamu-KIR1D, despite the difference in the number of animals studied per species (Tables I and II). These differences may indicate that KIR1D in cynomolgus macaques executes a more essential role.

On average, one more inhibitory KIR gene was present on haplotypes of rhesus macaques, whereas an additional activating KIR was encoded on cynomolgus macaque haplotypes. The differential gene and allele frequencies are indicators for speciesspecific selection, and might involve different infectious pathogen encounters due to varying habitats.

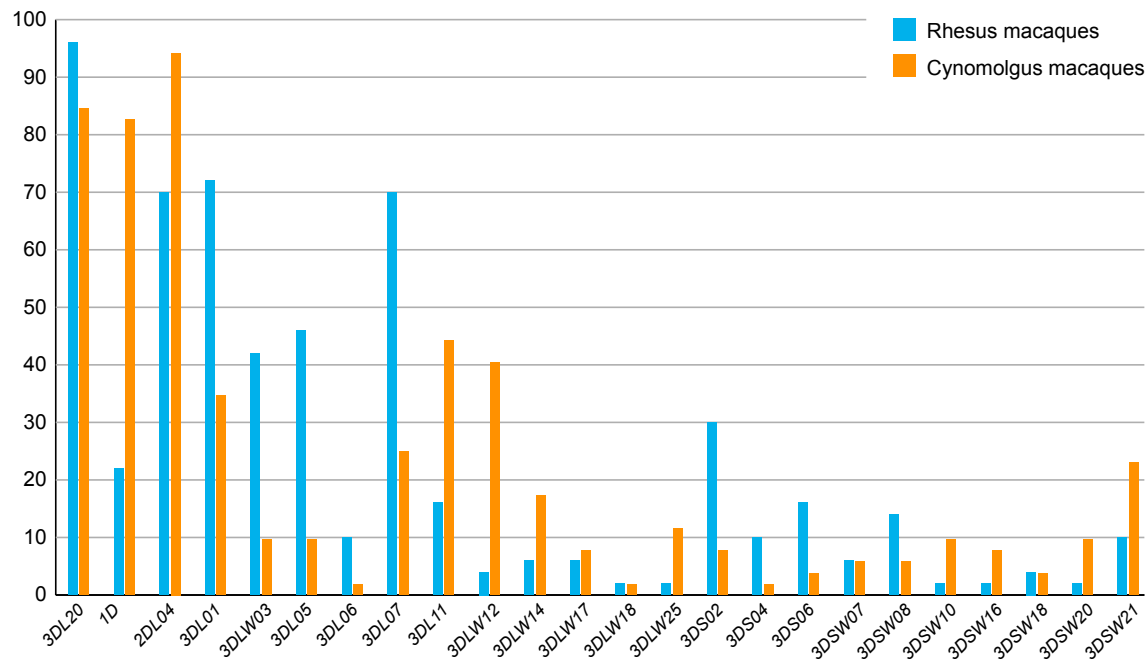

Figure 7. Gene frequencies of orthologous $K I R$ genes in rhesus and cynomolgus macaques. The gene frequencies are given for orthologous KIR genes in rhesus (blue bars) and cynomolgus (orange bars) macaques. 


\section{Differential KIR gene content and frequency in populations}

The populations of rhesus and cynomolgus macaques (Figure 1) parade differences in KIR gene content and gene frequency. Rhesus macaques from the Burmese population encoded, on average, one and two additional KIR3DL and KIR3DS receptors, respectively, as compared to the haplotypes that stem from the Indian and Chinese populations. Approximately $70 \%$ of the haplotypes contained at least one MamuKIR3DLO1 and/or Mamu-KIR3DL07 copy, regardless of the origin, whereas multiple other KIR genes were differently distributed over the rhesus macaque populations (Figure 8). For example, Mamu-KIR1D is located on $56 \%$ of the haplotypes from Burmese animals, whereas it is present on only $16 \%$ and $14 \%$ of the Indian and Chinese rhesus macaque KIR haplotypes, respectively. Eleven $K I R$ genes were identified in a single rhesus macaque population, including newly defined activating KIR3DS genes that were only encountered in the Burmese cohort studied.

In cynomolgus macaques, animals that originate from the mainland populations seem to have, on average, one additional inhibitory KIR receptor (KIR3DL) per haplotype as compared to the subjects that inhabit the Indonesian/Malaysian islands. Differential gene distribution trends are observed for several KIR genes (Figure 9). For example, Mafa-KIR3DLO1 and-KIR3DLW12, which were more frequently identified in the northern mainland population, and -KIR3DLW19, -KIR3DLW21,-KIR3DLW28, and $-K I R 3 D S W 13$, which were found present only in the Mauritian population. Activating $K I R$ genes with orthologs in Indian rhesus macaques were mainly identified in the northern mainland population, including KIR3DSO2 and KIR3DSW07, whereas the other cynomolgus macaque populations have species-specific activating KIR genes.

Genes that were identified in either three rhesus or four cynomolgus macaque populations mainly encode inhibitory receptors (Figures $\mathbf{8}$ and $\mathbf{9}$ ). The activating receptor are more often observed in two or one populations (Figures $\mathbf{8}$ and $\mathbf{9}$ ). Overall, the observed variable gene content and gene frequency in the different macaque populations support evidence pointing to rapid evolution of the KIR genes at the population level.

Figure 8. Gene distributions between the different rhesus macaque populations. Gene frequencies are listed for rhesus macaque KIR genes that were identified in the Indian (blue bars), Burmese (orange bars), and Chinese (purple bars) populations. The genes are listed from the most frequent genes in all populations to the least frequent species-specific genes. The background color indicates Mamu-KIR3DL20 (yellow), -KIR2DL04 (green), -KIR1D (light blue), and the inhibitory (blue) and activating (red) lineage II KIR genes. The frequencies are based on the presence on a haplotype of known origin rather than the presence in an individual. 


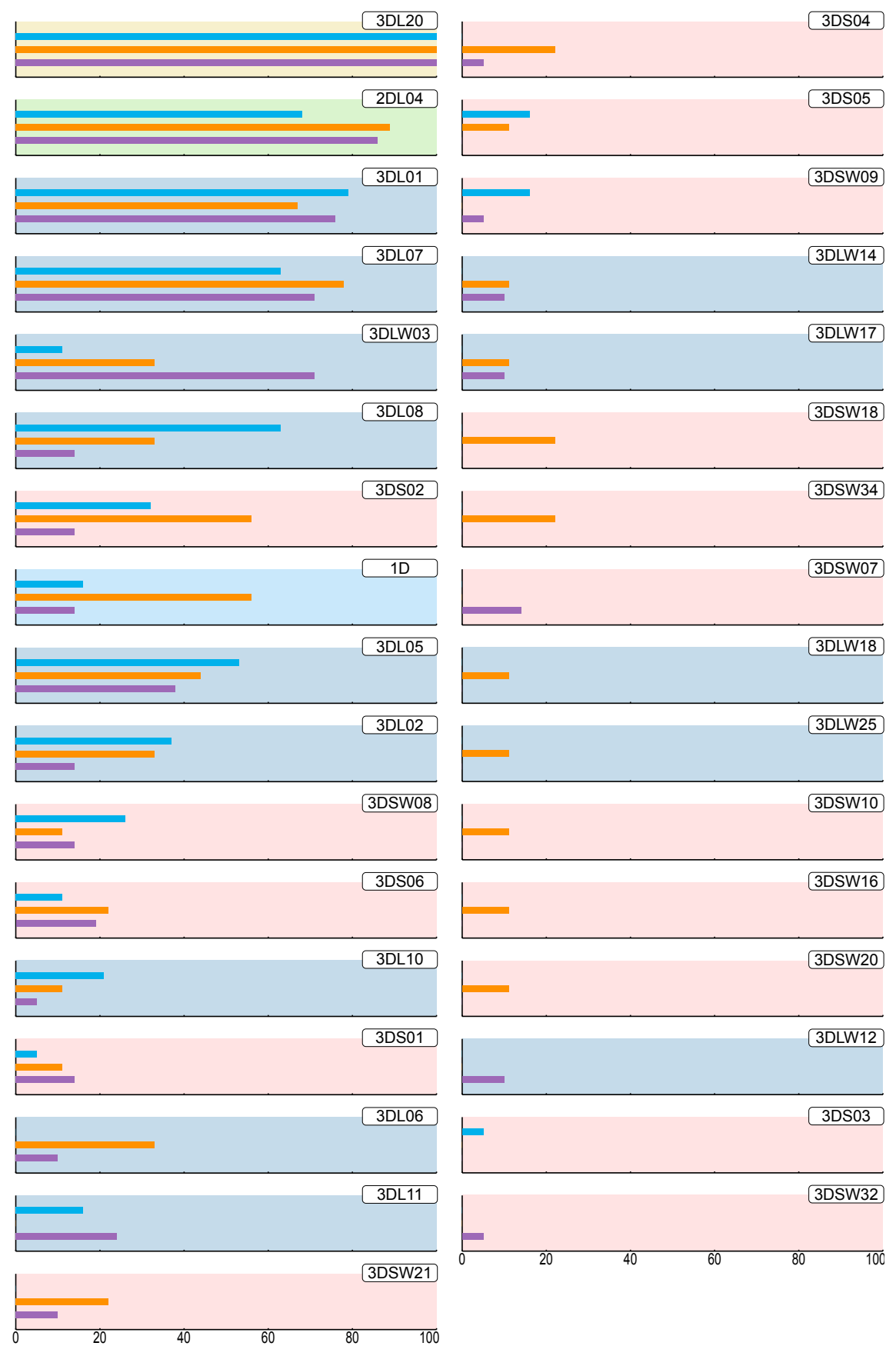

Indian Burmese Chinese 


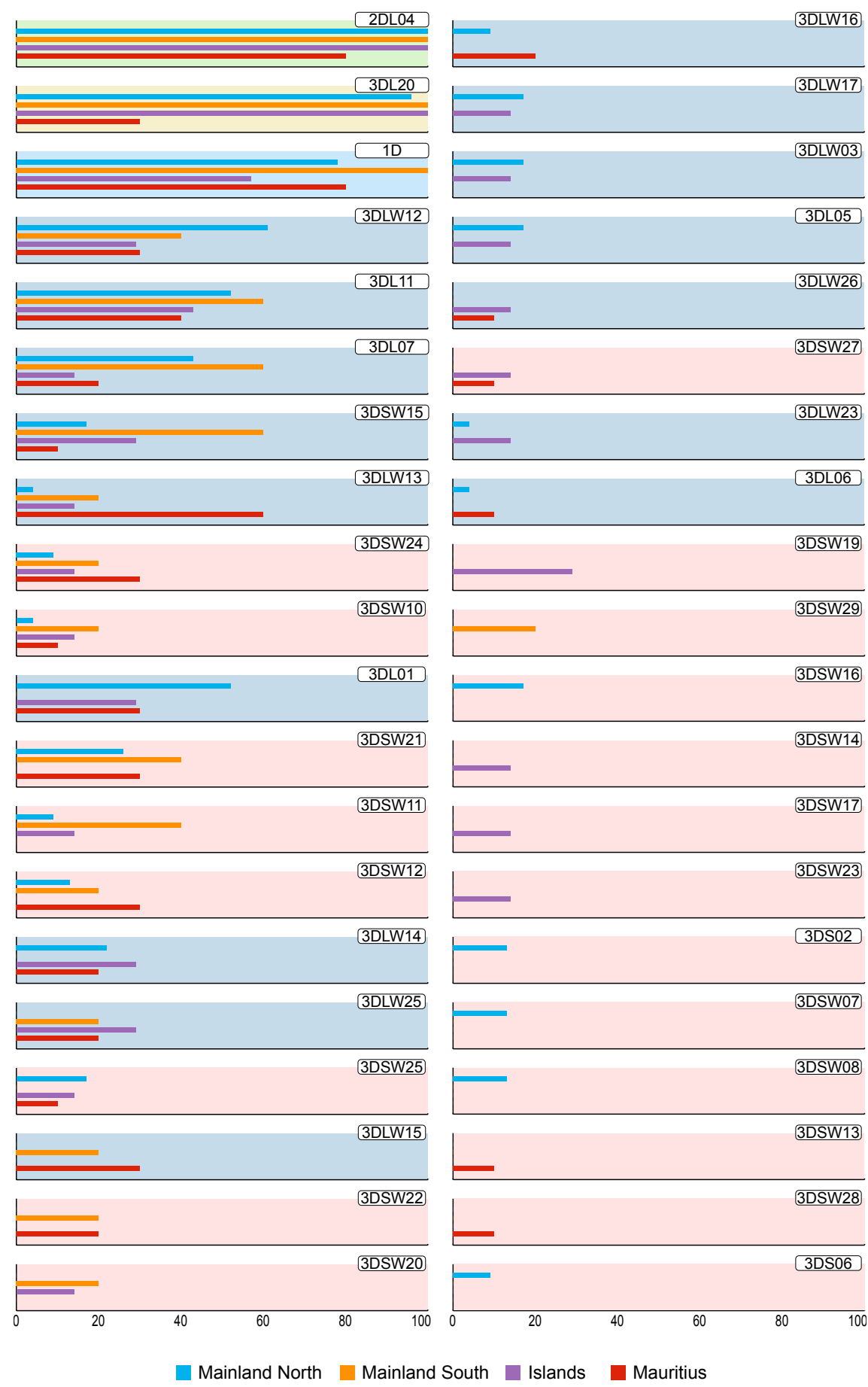




\section{Discussion}

An essential step in the evolution of the primate KIR cluster started with the initial expansion of a lineage II KIR gene progenitor. Subsequently, other KIR lineages seem to have emerged through deletion and recombination events. In macaques, lineage II KIR genes (KIR3D) were subjected to substantial expansion $(10,17,32)$, which coincides with an extended $M H C$ class I gene repertoire $(16,24)$. The present study involves the comparative analysis of rhesus and cynomolgus macaque populations from distinct geographic areas. The KIR gene repertoires were found to reflect rapid evolution. Our data illustrate that not only within these closely related species, but even within their populations, new KIR gene entities are generated by complex recombination processes resulting in the formation of hybrid genes. In addition, a high level of allelic polymorphism was encountered in both macaque species, but the sharing of alleles was virtually absent. Moreover, recombination resulted in marked differences in the $K I R$ haplotype architecture of both species, again testifying the rapid evolution of the macaque KIR genes, which has not been described in other non-human primate species.

In humans, the KIR gene cluster mainly diversifies at the allelic level, whereas gene expansion is modest and mainly confined to lineage III genes (43). Two major haplotype configurations are recognized, for which a trade-off has been suggested based on differential haplotype frequencies in human populations (47). The A haplotype configurations standardly express seven receptors and have an inhibitory profile, whereas the $B$ haplotypes show moderate gene content variability including multiple activating KIR genes (7 to 13 KIR genes) (Figure 10) (44). Chimpanzees (Pan troglodytes) and humans diverged from a common ancestor about 5 million years ago, and, although the complexity of the KIR clusters is to some extent comparable, speciesspecific diversification is observed in receptor structure, haplotype architecture, $M H C$ class I recognition potential, and gene content (48). The chimpanzee KIR region mainly comprises inhibitory genes, and resemble human A haplotypes. Several chimpanzee $K I R$ genes are actually recombinant genes (48). The repertoire, however, is limited to 13 KIR genes, four of which are orthologous framework genes that are shared with humans (Figure 10) $(41,48)$. Although little is known about the allelic variation in chimpanzees, the limited KIR gene repertoire might suggest that the ancient selective sweep, which targeted the ancestral chimpanzee MHC class I region and was likely caused by a retroviral infection $(49,50)$, may also have had an indirect impact on its ligands within the KIR gene region. Bonobos (Pan paniscus) and chimpanzees shared a common ancestor approximately 2.3 million years ago. In this species, only 7 KIR genes

4 Figure 9. Gene distributions between the different cynomolgus macaque populations. Frequencies are provided for KIR genes identified in cynomolgus macaque populations: mainland, north (blue bars) or south (orange bars) of the Isthmus of Kra, the Malaysian/Indonesian islands (purple bars), or Mauritius (red bars). The background color indicates Mamu-KIR3DL2O (yellow), -KIR2DLO4 (green), -KIR1D (light blue), and the inhibitory (blue) and activating (red) lineage II KIR genes. The frequencies are based on the presence of a gene on haplotype of known origin rather than on the presence in an individual. 


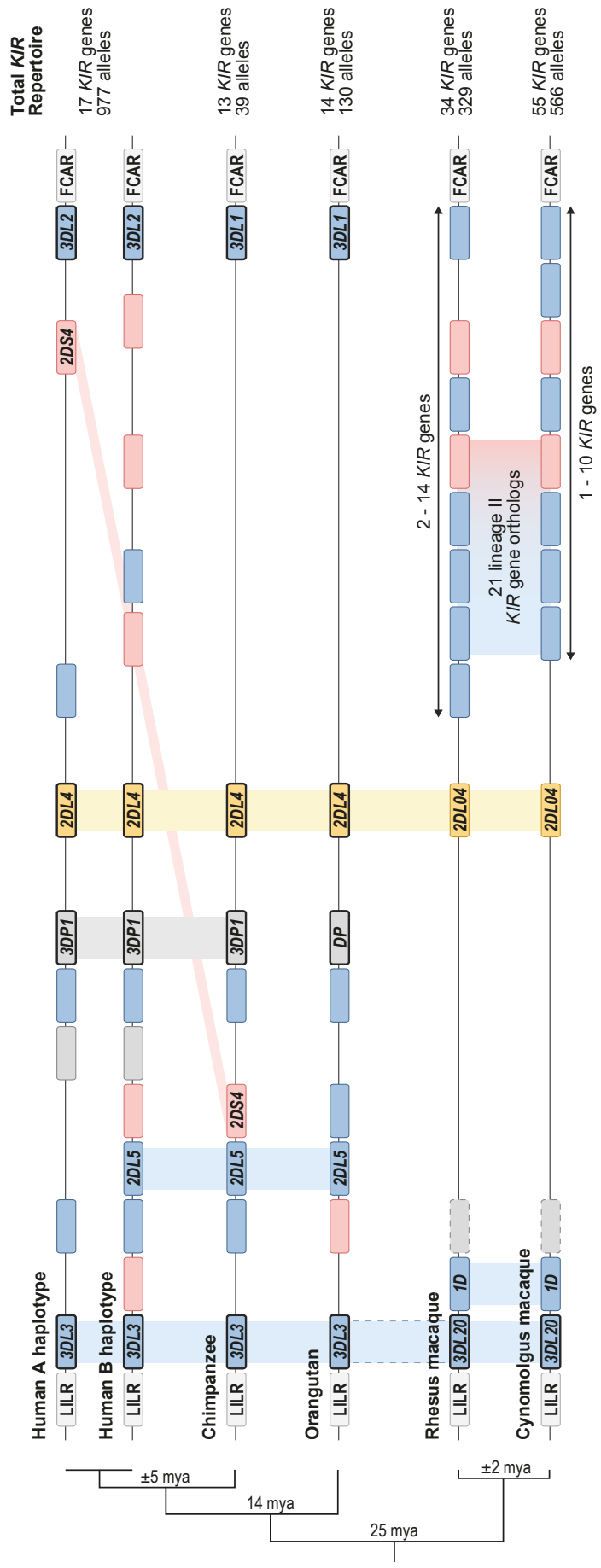

Figure 10. KIR haplotypes in different primate species. The KIR haplotype configurations of humans (A and B haplotypes), chimpanzees, orangutans, and rhesus and cynomolgus macaques are schematically illustrated. The evolutionary distance between the different species is depicted on the left, whereas the numbers of the documented KIR repertoires are provided on the right. The inhibitory and activating KIR genes are illustrated with blue and red boxes, whereas pseudogenes are in grey. Framework genes are indicated with black outlining, and orthologous and homologous genes shared in the depicted primate species are connected with each other. In total, 21 lineage II KIR genes are identified as orthologs in rhesus and cynomolgus macaques. KIR3DL20, identified as framework gene in both macaque species, is a potential ortholog of the other primate KIR3DL3 genes. A putative pseudogene on the centromeric macaque KIR haplotype is indicated by a grey box with a dashed outlining. The KIR region is flanked by the LILR and FCAR genes in all primate species depicted. 
are reported, 5 and 3 genes of which are orthologs, shared with common chimpanzees and humans, respectively (51). The short bonobo KIR haplotypes, the limited KIR gene repertoire, and the reduced bonobo $M H C$ class I content (52-55) may result from subsequent selective sweeps (56). The expansion of lineage III KIR genes, which in macaques are represented by KIR1D, correlates with the emergence of HLA-C-like genes in orangutans (57-59). In orangutans, 13 KIR genes are identified, of which the framework genes share orthologs with humans $(15,41,57,58)$. Two sibling orangutan species inhabit Sumatra (Pongo abelii) and Borneo (Pongo pygmaeus) (60). Only one gene is species-specific (KIR2DS15 in Bornean orangutans), whereas all other genes are orthologs. Ten of the 130 KIR alleles that were identified are shared between both sister species. For the human and great ape species discussed above, rapid evolution is mainly reflected by the gain in allelic variation, whereas the generation of novel gene entities and the formation of complex haplotype architectures seem to be relatively limited (Figure 10).

The present communication sheds light on the evolution of the KIR region in two highly related Old World monkey species, which share an introgression zone. In rhesus and cynomolgus macaques, the massive expansion of the lineage II KIR genes exceeds the modest lineage III expansion in great apes and humans. The rapid evolution of the macaque KIR region is reflected not only by allelic polymorphism but even more prominently by the large number of species-specific recombinant genes and haplotypes with a complex architecture in different macaque populations. In humans and other hominids, these events seem to be less abundant. Moreover, the allelic variation in macaques seems to exceed the numbers that are encountered in humans and higher primates. We recorded a total number of 579 KIR alleles, and it is important to realize that the number of samples that were analyzed is relatively small as compared to the human situation (43). The reason for the extensive expansion of the KIR gene cluster in macaques is of interest. Whereas in humans the less variable $K I R$ gene content and haplotype configurations seem to be the result of a trade-off, such an indication appears to be absent in macaques. The most plausible driving forces of the rapid KIR cluster evolution in macaques might involve co-evolution with the extended $\mathrm{MHC}$ class I region, differential infectious pathogen encounters, a discontinuous habitat, and susceptibility to chromosomal recombination. Nonetheless, we cannot rule out that the extensive expansion of the macaque KIR gene system may have evolved due to the lack of evolutionary pressure on this system. The ligand of only a few receptors have been identified, and therefore the functional impact of the expanded macaque KIR repertoire remains largely unclear. However, diversification of ligand interactions is suggested by overlapping, but non-redundant, MHC class I specificity of multiple KIR (61-66). The extensive diversity of the macaque MHC and KIR clusters might facilitate interactions with allele-level specificity, differential affinity, and peptide dependency, and may contribute to rapid adaption driven by environmental conditions.

The general high levels of allelic polymorphism detected in the KIR region in primates might indicate that it is more prone to generate mutations than other regions of the genome. Mutation rates are elevated in $\mathrm{CpG}$ islands, which are genomic regions 
that are enriched for $\mathrm{CpG}$ sites with an observed-to-expected ratio greater than 60 percent. All KIR genes indeed carry CpG islands (67). CpG site mutations, however, mainly involve cytosine to thymine transitions, whereas mutations in the generation of the novel Mamu-KIR3DLO5 allele involved T > C and G > T transitions, which are not commonly observed transition events. The two point mutations are separated by only two nucleotides, which suggests that one mutation initiated the other, and perhaps was caused by the recruitment of error-prone repair mechanisms (68). In addition to CpG islands and error-prone repair, other factors that might enhance the regional mutation rate may include recombination events, deletion and insertion events, chromatin configurations, distance to the telomere, and replication time $(69,70)$. Furthermore, relatively more single nucleotide polymorphisms were observed in regions that were homologous in humans, chimpanzees, and macaques, which substantiates the extensive variation of the KIR gene region (69). The birth of novel KIR alleles has been described previously in human families (71), and, together with the event recorded in macaques, this might suggest that point mutations substantially contribute to the extensive allelic KIR variation. Of course, it is clear that the generation of mutations is only one side of the coin, and that selection determines which polymorphisms will be enriched in the populations or are eventually rooted out.

In humans, genetic KIR variation is documented for over 250 populations and mainly records allelic variation and differential haplotype distribution in relation to gene frequencies $(47,72)$. Similar observations were made for the different macaque populations. Genes that are shared in all three rhesus or four cynomolgus macaque populations mainly involved inhibitory KIR genes (Figures 8 and 9). The conserved nature of these genes suggests an impact on essential functions, such as NK cell education, a process for which the involvement of inhibitory KIR is well established in humans (73-75). The role of activating KIR in humans is less understood, but associations with disease progression or protection are described, and in vitro studies demonstrate specific binding to certain peptide-MHC class I complexes (76-81). In macaques, activating $K I R$ genes were mainly identified in one or two populations and may substantiate more specialized functions, like pathogen recognition (Figures $\mathbf{8}$ and $\mathbf{9}$ ). For instance, Mamu-KIR3DSW18 is encountered only in Burmese rhesus macaques, and a similar observation was made for Mafa-KIR3DSW08 in the northern-mainland cynomolgus macaques. In addition, the majority of the KIR alleles appear not to be shared at the population level (Figure 4). Again, this hints at a speedy generation of allelic polymorphism. For the Indian rhesus macaque population, a genetic bottleneck is evident (82), but it did not result in a reduced KIR gene content, which might indicate that the rapid evolution of the KIR repertoire erased the genetic footprint of a bottleneck.

Rhesus and cynomolgus macaques are widely used as preclinical models in translational biomedical research in order to further a better understanding of human diseases and the development of vaccines and therapies $(34,35)$. The genetic makeup of the different macaque species, however, can vary considerably and might potentially influence the outcome of studies. Even at the population level, a differential disease 
susceptibility has been reported: for example, in SIV/AIDS-related experiments in rhesus macaques of Indian and Chinese origin $(36,37,83)$. It is possible that the $K I R$ repertoire may be one of the factors that have an impact on disease outcome, as correlations between KIR gene content and disease phenotypes in humans (6) and macaques are documented $(38,39)$.

This study design, including rhesus and cynomolgus macaque families from different geographical origin, allowed the transcriptomic characterization of the complex KIR cluster. The high level of allelic polymorphism, the number of novel gene entities, the plastic haplotype architecture, and the diversification at the species and population levels illustrate the unparalleled rapid evolution of the KIR gene region in macaques. This communication paves the way to study the impact of $K I R$ genes in nonhuman primate models for human health and disease, but also may help in selecting animals with particular genetic markers for studies in the area of personalized medicine.

\section{Acknowledgements}

We thank D. Devine for editing the manuscript, and F. van Hassel for preparing the figures.

\section{Materials \& data}

Requests for materials and data can be addressed to Prof. Dr. Ronald E. Bontrop (bontrop@bprc.nl). The sequences presented in this article have been submitted to the European Nucleotide Archive (https://www.ebi.ac.uk/ena/) under accession number PRJEB33481. 


\section{References}

1. Benson, DM, Jr., Caligiuri MA. 2014. Killer immunoglobulin-like receptors and tumor immunity. Cancer immunology research 2(2):99-104.

2. Ljunggren, HG, Karre K. 1990. In search of the 'missing self': MHC molecules and NK cell recognition. Immunology today 11(7):237-244.

3. Lanier, LL. 2005. NK cell recognition. Annual review of immunology 23:225-274.

4. Battistini, L, Borsellino G, Sawicki G, Poccia F, Salvetti M, Ristori G, Brosnan CF. 1997. Phenotypic and cytokine analysis of human peripheral blood gamma delta T cells expressing NK cell receptors. Journal of immunology (Baltimore, Md : 1950) 159(8):3723-3730.

5. Trowsdale, J. 2001. Genetic and functional relationships between MHC and NK receptor genes. Immunity 15(3):363-374.

6. Kulkarni, S, Martin MP, Carrington M. 2008. The Yin and Yang of HLA and KIR in human disease. Seminars in immunology 20(6):343-352.

7. Parham, P. 2005. MHC class I molecules and KIRs in human history, health and survival. Nature reviews Immunology 5(3):201-214.

8. Trowsdale, J, Barten R, Haude A, Stewart CA, Beck S, Wilson MJ. 2001. The genomic context of natural killer receptor extended gene families. Immunological reviews 181:20-38.

9. Hsu, KC, Chida S, Geraghty DE, Dupont B. 2002. The killer cell immunoglobulin-like receptor (KIR) genomic region: gene-order, haplotypes and allelic polymorphism. Immunological reviews 190:40-52.

10. Bruijnesteijn, J, van der Wiel MKH, Swelsen WTN, Otting N, de Vos-Rouweler AJM, Elferink D, Doxiadis GG, Claas FHJ, Lardy NM, de Groot NG, Bontrop RE. 2018. Human and Rhesus Macaque KIR Haplotypes Defined by Their Transcriptomes. Journal of immunology (Baltimore, Md : 1950) 200(5):1692-1701.

11. Bruijnesteijn, J, van der Wiel MKH, de Groot N, Otting N, de Vos-Rouweler AJM, Lardy NM, de Groot NG, Bontrop RE. 2018. Extensive Alternative Splicing of KIR Transcripts. Frontiers in Immunology 9(2846).

12. Martin, AM, Freitas EM, Witt CS, Christiansen FT. 2000. The genomic organization and evolution of the natural killer immunoglobulin-like receptor (KIR) gene cluster. Immunogenetics 51(4-5):268-280.

13. Carrillo-Bustamante, P, Kesmir C, de Boer RJ. 2016. The evolution of natural killer cell receptors. Immunogenetics 68(1):3-18.

14. Parham, P, Norman PJ, Abi-Rached L, Guethlein LA. 2012. Human-specific evolution of killer cell immunoglobulin-like receptor recognition of major histocompatibility complex class I molecules. Philosophical transactions of the Royal Society of London Series B, Biological sciences 367(1590):800-811.

15. Guethlein, LA, Norman PJ, Heijmans CM, de Groot NG, Hilton HG, Babrzadeh F, Abi-Rached L, Bontrop RE, Parham P. 2017. Two Orangutan Species Have Evolved Different KIR Alleles and Haplotypes. Journal of immunology (Baltimore, Md : 1950) 198(8):3157-3169.

16. Daza-Vamenta, R, Glusman G, Rowen L, Guthrie B, Geraghty DE. 2004. Genetic divergence of the rhesus macaque major histocompatibility complex. Genome research 14(8):1501-1515.

17. de Groot, NG, Blokhuis JH, Otting N, Doxiadis GG, Bontrop RE. 2015. Co-evolution of the MHC class I and KIR gene families in rhesus macaques: ancestry and plasticity. Immunological reviews 267(1):228-245.

18. Kanthaswamy, S, Ng J, Satkoski Trask J, George DA, Kou AJ, Hoffman LN, Doherty TB, Houghton P, Smith DG. 2013. The genetic composition of populations of cynomolgus macaques (Macaca fascicularis) used in biomedical research. Journal of medical primatology 42(3):120-131.

19. Blancher, A, Bonhomme M, Crouau-Roy B, Terao K, Kitano T, Saitou N. 2008. Mitochondrial DNA sequence phylogeny of 4 populations of the widely distributed cynomolgus macaque (Macaca fascicularis fascicularis). The Journal of heredity 99(3):254-264.

20. Bunlungsup, S, Kanthaswamy S, Oldt RF, Smith DG, Houghton P, Hamada Y, Malaivijitnond S. 2017. Genetic analysis of samples from wild populations opens new perspectives on hybridization between long-tailed (Macaca fascicularis) and rhesus macaques (Macaca mulatta). American journal of primatology 79(12).

21. Stevison, LS, Kohn MH. 2009. Divergence population genetic analysis of hybridization between rhesus and cynomolgus macaques. Molecular ecology 18(11):2457-2475. 
22. Yan, G, Zhang G, Fang X, Zhang Y, Li C, Ling F, Cooper DN, Li Q, Li Y, van Gool AJ, Du H, Chen J, Chen R, Zhang P, Huang Z, Thompson JR, Meng Y, Bai Y, Wang J, Zhuo M, Wang T, Huang Y, Wei L, Li J, Wang Z, Hu H, Yang P, Le L, Stenson PD, Li B, Liu X, Ball EV, An N, Huang Q, Zhang Y, Fan W, Zhang X, Li Y, Wang W, Katze MG, Su B, Nielsen $\mathrm{R}$, Yang $\mathrm{H}$, Wang J, Wang $X$, Wang J. 2011. Genome sequencing and comparison of two nonhuman primate animal models, the cynomolgus and Chinese rhesus macaques. Nature Biotechnology 29:1019.

23. Karl, JA, Bohn PS, Wiseman RW, Nimityongskul FA, Lank SM, Starrett GJ, O'Connor DH. 2013. Major histocompatibility complex class I haplotype diversity in Chinese rhesus macaques. G3 (Bethesda, Md) 3(7):1195-1201.

24. Otting, N, Heijmans CM, Noort RC, de Groot NG, Doxiadis GG, van Rood JJ, Watkins DI, Bontrop RE. 2005 Unparalleled complexity of the MHC class I region in rhesus macaques. Proceedings of the National Academy of Sciences of the United States of America 102(5):1626-1631.

25. Doxiadis, GG, Rouweler AJ, de Groot NG, Louwerse A, Otting N, Verschoor EJ, Bontrop RE. 2006. Extensive sharing of MHC class II alleles between rhesus and cynomolgus macaques. Immunogenetics 58(4):259-268.

26. Blokhuis, JH, van der Wiel MK, Doxiadis GG, Bontrop RE. 2010. The mosaic of KIR haplotypes in rhesus macaques. Immunogenetics 62(5):295-306.

27. Blokhuis, JH, van der Wiel MK, Doxiadis GG, Bontrop RE. 2011. The extreme plasticity of killer cell Ig-like receptor (KIR) haplotypes differentiates rhesus macaques from humans. European journal of immunology 41(9):2719-2728

28. Sambrook, JG, Bashirova A, Palmer S, Sims S, Trowsdale J, Abi-Rached L, Parham P, Carrington M, Beck S. 2005 Single haplotype analysis demonstrates rapid evolution of the killer immunoglobulin-like receptor (KIR) loci in primates. Genome research 15(1):25-35.

29. Kruse, PH, Rosner C, Walter L. 2010. Characterization of rhesus macaque KIR genotypes and haplotypes. Immunogenetics 62(5):281-293.

30. Moreland, AJ, Guethlein LA, Reeves RK, Broman KW, Johnson RP, Parham P, O'Connor DH, Bimber BN. 2011. Characterization of killer immunoglobulin-like receptor genetics and comprehensive genotyping by pyrosequencing in rhesus macaques. BMC genomics 12:295.

31. Bimber, BN, Moreland AJ, Wiseman RW, Hughes AL, O'Connor DH. 2008. Complete characterization of killer Ig-like receptor (KIR) haplotypes in Mauritian cynomolgus macaques: novel insights into nonhuman primate KIR gene content and organization. Journal of immunology (Baltimore, Md : 1950) 181(9):6301-6308.

32. Bimber, BN, Evans DT. 2015. The killer-cell immunoglobulin-like receptors of macaques. Immunological reviews 267(1):246-258.

33. Prall, TM, Graham ME, Karl JA, Wiseman RW, Ericsen AJ, Raveendran M, Alan Harris R, Muzny DM, Gibbs RA, Rogers J, O'Connor DH. 2017. Improved full-length killer cell immunoglobulin-like receptor transcript discovery in Mauritian cynomolgus macaques. Immunogenetics 69(5):325-339.

34. Dijkman, K, Sombroek CC, Vervenne RAW, Hofman SO, Boot C, Remarque EJ, Kocken CHM, Ottenhoff THM, Kondova I, Khayum MA, Haanstra KG, Vierboom MPM, Verreck FAW. 2019. Prevention of tuberculosis infection and disease by local BCG in repeatedly exposed rhesus macaques. Nature medicine 25(2):255-262.

35. Harding, JD. 2017. Genomic Tools for the Use of Nonhuman Primates in Translational Research. ILAR journal 58(1):59-68.

36. Burdo, TH, Marcondes MC, Lanigan CM, Penedo MC, Fox HS. 2005. Susceptibility of Chinese rhesus monkeys to SIV infection. AIDS (London, England) 19(15):1704-1706

37. Ling, B, Veazey RS, Luckay A, Penedo C, Xu K, Lifson JD, Marx PA. 2002. SIV(mac) pathogenesis in rhesus macaques of Chinese and Indian origin compared with primary HIV infections in humans. AIDS (London, England) 16(11):1489-1496.

38. Albrecht, C, Malzahn D, Brameier M, Hermes M, Ansari AA, Walter L. 2014. Progression to AIDS in SIV-Infected Rhesus Macaques is Associated with Distinct KIR and MHC class I Polymorphisms and NK Cell Dysfunction. Front Immunol 5:600.

39. Walter, L, Ansari AA. 2015. MHC and KIR Polymorphisms in Rhesus Macaque SIV Infection. Front Immunol 6:540.

40. Doxiadis, GG, de Groot N, de Groot NG, Rotmans G, de Vos-Rouweler AJ, Bontrop RE. 2010. Extensive DRB region diversity in cynomolgus macaques: recombination as a driving force. Immunogenetics 62(3):137-147. 
41. Robinson, J, Guethlein LA, Maccari G, Blokhuis J, Bimber BN, de Groot NG, Sanderson ND, Abi-Rached L, Walter L, Bontrop RE, Hammond JA, Marsh SGE, Parham P. 2018. Nomenclature for the KIR of non-human species. Immunogenetics 70(9):571-583.

42. Doxiadis, GG, de Groot N, Otting N, Blokhuis JH, Bontrop RE. 2011. Genomic plasticity of the MHC class I A region in rhesus macaques: extensive haplotype diversity at the population level as revealed by microsatellites. Immunogenetics 63(2):73-83.

43. Robinson, J, Halliwell JA, McWilliam H, Lopez R, Marsh SG. 2013. IPD--the Immuno Polymorphism Database. Nucleic acids research 41(Database issue):D1234-1240.

44. Uhrberg, M, Valiante NM, Shum BP, Shilling HG, Lienert-Weidenbach K, Corliss B, Tyan D, Lanier LL, Parham P. 1997. Human diversity in killer cell inhibitory receptor genes. Immunity 7(6):753-763.

45. Roe, D, Vierra-Green C, Pyo CW, Eng K, Hall R, Kuang R, Spellman S, Ranade S, Geraghty DE, Maiers M. 2017. Revealing complete complex KIR haplotypes phased by long-read sequencing technology. Genes and immunity 18(3):127-134

46. Bruijnesteijn, J, de Groot NG, Otting N, Maccari G, Guethlein LA, Robinson J, Marsh SGE, Walter L, O'Connor DH, Hammond JA, Parham P, Bontrop RE. 2019. Nomenclature report for killer-cell immunoglobulin-like receptors (KIR) in macaque species: new genes/alleles, renaming recombinant entities and IPD-NHKIR updates. Immunogenetics.

47. Middleton, D, Gonzelez F. 2010. The extensive polymorphism of KIR genes. Immunology 129(1):8-19.

48. Abi-Rached, L, Moesta AK, Rajalingam R, Guethlein LA, Parham P. 2010. Human-specific evolution and adaptation led to major qualitative differences in the variable receptors of human and chimpanzee natural killer cells. PLoS genetics 6(11):e1001192.

49. de Groot, NG, Otting N, Doxiadis GGM, Balla-Jhagjhoorsingh SS, Heeney JL, van Rood JJ, Gagneux P, Bontrop RE. 2002. Evidence for an ancient selective sweep in the MHC class I gene repertoire of chimpanzees. Proceedings of the National Academy of Sciences 99(18):11748-11753.

50. de Groot, NG, Heijmans CM, Zoet YM, de Ru AH, Verreck FA, van Veelen PA, Drijfhout JW, Doxiadis GG, Remarque EJ, Doxiadis, II, van Rood JJ, Koning F, Bontrop RE. 2010. AIDS-protective HLA-B*27/B*57 and chimpanzee MHC class I molecules target analogous conserved areas of HIV-1/SIVcpz. Proceedings of the National Academy of Sciences of the United States of America 107(34):15175-15180.

51. Rajalingam, R, Hong M, Adams EJ, Shum BP, Guethlein LA, Parham P. 2001. Short KIR haplotypes in pygmy chimpanzee (Bonobo) resemble the conserved framework of diverse human KIR haplotypes. The Journal of experimental medicine 193(1):135-146.

52. Wroblewski, EE, Guethlein LA, Norman PJ, Li Y, Shaw CM, Han AS, Ndjango JN, Ahuka-Mundeke S, Georgiev AV, Peeters M, Hahn BH, Parham P. 2017. Bonobos Maintain Immune System Diversity with Three Functional Types of MHC-B. Journal of immunology (Baltimore, Md : 1950) 198(9):3480-3493.

53. Maibach, V, Hans JB, Hvilsom C, Marques-Bonet T, Vigilant L. 2017. MHC class I diversity in chimpanzees and bonobos. Immunogenetics 69(10):661-676.

54. Maibach, V, Vigilant L. 2019. Reduced bonobo MHC class I diversity predicts a reduced viral peptide binding ability compared to chimpanzees. BMC evolutionary biology 19(1):14.

55. de Groot, NG, Heijmans CMC, Helsen P, Otting N, Pereboom Z, Stevens JMG, Bontrop RE. 2017. Limited MHC class I intron 2 repertoire variation in bonobos. Immunogenetics 69(10):677-688.

56. de Groot, NG, Stevens JMG, Bontrop RE. 2018. Does the MHC Confer Protection against Malaria in Bonobos? Trends in immunology 39(10):768-771.

57. Guethlein, LA, Flodin LR, Adams EJ, Parham P. 2002. NK cell receptors of the orangutan (Pongo pygmaeus): a pivotal species for tracking the coevolution of killer cell Ig-like receptors with MHC-C. Journal of immunology (Baltimore, Md : 1950) 169(1):220-229.

58. Guethlein, LA, Older Aguilar AM, Abi-Rached L, Parham P. 2007. Evolution of killer cell Ig-like receptor (KIR) genes: definition of an orangutan KIR haplotype reveals expansion of lineage III KIR associated with the emergence of MHC-C. Journal of immunology (Baltimore, Md : 1950) 179(1):491-504.

59. de Groot, NG, Heijmans CM, van der Wiel MK, Blokhuis JH, Mulder A, Guethlein LA, Doxiadis GG, Claas FH, Parham P, Bontrop RE. 2016. Complex MHC Class I Gene Transcription Profiles and Their Functional Impact in Orangutans. Journal of immunology (Baltimore, Md : 1950) 196(2):750-758. 
60. Steiper, ME. 2006. Population history, biogeography, and taxonomy of orangutans (Genus: Pongo) based on a population genetic meta-analysis of multiple loci. Journal of human evolution 50(5):509-522.

61. Ries, M, Reynolds MR, Bashkueva K, Crosno K, Capuano S, 3rd, Prall TM, Wiseman R, O'Connor DH, Rakasz EG, Uno H, Lifson JD, Evans DT. 2017. KIR3DL01 upregulation on gut natural killer cells in response to SIV infection of KIR- and MHC class I-defined rhesus macaques. PLoS pathogens 13(7):e1006506.

62. Banerjee, P, Ries M, Janaka SK, Grandea AG, 3rd, Wiseman R, O'Connor DH, Golos TG, Evans DT. 2018. Diversification of Bw4 Specificity and Recognition of a Nonclassical MHC Class I Molecule Implicated in Maternal-Fetal Tolerance by Killer Cell Ig-like Receptors of the Rhesus Macaque. Journal of immunology (Baltimore, Md : 1950) 201(9):2776-2786.

63. Schafer, JL, Colantonio AD, Neidermyer WJ, Dudley DM, Connole M, O'Connor DH, Evans DT. 2014. KIR3DL01 recognition of Bw4 ligands in the rhesus macaque: maintenance of Bw4 specificity since the divergence of apes and Old World monkeys. Journal of immunology (Baltimore, Md : 1950) 192(4):1907-1917.

64. Colantonio, AD, Bimber BN, Neidermyer WJ, Jr., Reeves RK, Alter G, Altfeld M, Johnson RP, Carrington M, O'Connor DH, Evans DT. 2011. KIR polymorphisms modulate peptide-dependent binding to an MHC class I ligand with a Bw6 motif. PLoS pathogens 7(3):e1001316.

65. Maloveste, SM, Chen D, Gostick E, Vivian JP, Plishka RJ, Iyengar R, Kruthers RL, Buckler-White A, Brooks AG, Rossjohn J, Price DA, Lafont BA. 2012. Degenerate recognition of MHC class I molecules with Bw4 and Bw6 motifs by a killer cell Ig-like receptor 3DL expressed by macaque NK cells. Journal of immunology (Baltimore, Md : 1950) 189(9):4338-4348

66. Rosner, C, Kruse PH, Hermes M, Otto N, Walter L. 2011. Rhesus macaque inhibitory and activating KIR3D interact with Mamu-A-encoded ligands. Journal of immunology (Baltimore, Md : 1950) 186(4):2156-2163.

67. Santourlidis, S, Trompeter HI, Weinhold S, Eisermann B, Meyer KL, Wernet P, Uhrberg M. 2002. Crucial role of DNA methylation in determination of clonally distributed killer cell Ig-like receptor expression patterns in NK cells. Journal of immunology (Baltimore, Md: 1950) 169(8):4253-4261.

68. Walser, JC, Ponger L, Furano AV. 2008. CpG dinucleotides and the mutation rate of non-CpG DNA. Genome research 18(9):1403-1414

69. Hodgkinson, A, Eyre-Walker A. 2011. Variation in the mutation rate across mammalian genomes. Nature reviews Genetics 12(11):756-766.

70. Zhu, Y, Neeman T, Yap VB, Huttley GA. 2017. Statistical Methods for Identifying Sequence Motifs Affecting Point Mutations. Genetics 205(2):843-856.

71. Halfpenny, IA, Middleton D, Barnett YA, Williams F. 2004. Investigation of killer cell immunoglobulin-like receptor gene diversity: IV. KIR3DL1/S1. Human immunology 65(6):602-612.

72. Gonzalez-Galarza, FF, Takeshita LY, Santos EJ, Kempson F, Maia MH, da Silva AL, Teles e Silva AL, Ghattaoraya GS, Alfirevic A, Jones AR, Middleton D. 2015. Allele frequency net 2015 update: new features for HLA epitopes, KIR and disease and HLA adverse drug reaction associations. Nucleic acids research 43(Database issue):D784-788.

73. Anfossi, N, Andre P, Guia S, Falk CS, Roetynck S, Stewart CA, Breso V, Frassati C, Reviron D, Middleton D, Romagne F, Ugolini S, Vivier E. 2006. Human NK cell education by inhibitory receptors for MHC class I. Immunity 25(2):331-342.

74. Yawata, M, Yawata N, Draghi M, Partheniou F, Little AM, Parham P. 2008. MHC class I-specific inhibitory receptors and their ligands structure diverse human NK-cell repertoires toward a balance of missing selfresponse. Blood 112(6):2369-2380.

75. Kim, S, Poursine-Laurent J, Truscott SM, Lybarger L, Song YJ, Yang L, French AR, Sunwoo JB, Lemieux S, Hansen TH, Yokoyama WM. 2005. Licensing of natural killer cells by host major histocompatibility complex class I molecules. Nature 436(7051):709-713.

76. Sim, MJW, Rajagopalan S, Altmann DM, Boyton RJ, Sun PD, Long EO. 2019. Human NK cell receptor KIR2DS4 detects a conserved bacterial epitope presented by HLA-C. Proceedings of the National Academy of Sciences of the United States of America 116(26):12964-12973.

77. Venstrom, JM, Pittari G, Gooley TA, Chewning JH, Spellman S, Haagenson M, Gallagher MM, Malkki M, Petersdorf E, Dupont B, Hsu KC. 2012. HLA-C-Dependent Prevention of Leukemia Relapse by Donor Activating KIR2DS1. New England Journal of Medicine 367(9):805-816.

78. Chewning, JH, Gudme CN, Hsu KC, Selvakumar A, Dupont B. 2007. KIR2DS1-positive NK cells mediate alloresponse against the C2 HLA-KIR ligand group in vitro. Journal of immunology (Baltimore, Md : 1950) 179(2):854-868. 
Chapter 3 | Unparalleled rapid evolution of KIR genes in macaque species

79. Stewart, CA, Laugier-Anfossi F, Vely F, Saulquin X, Riedmuller J, Tisserant A, Gauthier L, Romagne F, Ferracci G, Arosa FA, Moretta A, Sun PD, Ugolini S, Vivier E. 2005. Recognition of peptide-MHC class I complexes by activating killer immunoglobulin-like receptors. Proceedings of the National Academy of Sciences of the United States of America 102(37):13224-13229.

80. Ivarsson, MA, Michaëlsson J, Fauriat C. 2014. Activating Killer Cell Ig-Like Receptors in Health and Disease. Frontiers in Immunology 5(184)

81. Martin, MP, Gao X, Lee JH, Nelson GW, Detels R, Goedert JJ, Buchbinder S, Hoots K, Vlahov D, Trowsdale J, Wilson M, O'Brien SJ, Carrington M. 2002. Epistatic interaction between KIR3DS1 and HLA-B delays the progression to AIDS. Nature genetics 31(4):429-434.

82. Xue, C, Raveendran M, Harris RA, Fawcett GL, Liu X, White S, Dahdouli M, Rio Deiros D, Below JE, Salerno W, Cox L, Fan G, Ferguson B, Horvath J, Johnson Z, Kanthaswamy S, Kubisch HM, Liu D, Platt M, Smith DG, Sun B, Vallender EJ, Wang F, Wiseman RW, Chen R, Muzny DM, Gibbs RA, Yu F, Rogers J. 2016. The population genomics of rhesus macaques (Macaca mulatta) based on whole-genome sequences. Genome research 26(12):1651-1662.

83. Sui, Y, Gordon S, Franchini G, Berzofsky JA. 2013. Nonhuman primate models for HIV/AIDS vaccine development. Current protocols in immunology 102:Unit 12.14. 




\section{Chapter

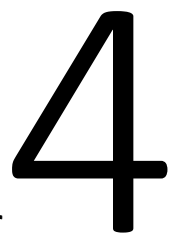 \\ Extensive alternative splicing of KIR transcripts}

Jesse Bruijnesteijn ${ }^{1 *}$, Marit K. H. van der Wiel ${ }^{1}$, Nanine de Groot ${ }^{1}$, Nel Otting ${ }^{1}$, Annemiek J. M. de Vos-Rouweler ${ }^{1}$, Neubury M. Lardy ${ }^{2}$, Natasja G. de Groot ${ }^{1}$, and Ronald E. Bontrop ${ }^{1,3}$

${ }^{1}$ Comparative Genetics and Refinement,

Biomedical Primate Research Centre, Rijswijk, The Netherlands ${ }^{2}$ Dept. of Immunogenetics, Sanquin, Amsterdam, The Netherlands ${ }^{3}$ Theoretical Biology and Bioinformatics, Utrecht University, Utrecht, The Netherlands

*Corresponding author: Jesse Bruijnesteijn Bruijnesteijn@bprc.nl Tel. nr. +31-15-2842574 Fax nr. $+31-15-2842600$ 


\begin{abstract}
The killer-cell Ig-like receptors (KIR) form a multigene entity involved in modulating immune responses through interactions with $\mathrm{MHC}$ class I molecules. The complexity of the KIR cluster is reflected by, for instance, abundant levels of allelic polymorphism, gene copy number variation, and stochastic expression profiles. The current transcriptome study involving human and macaque families demonstrates that KIR family members are also subjected to differential levels of alternative splicing, and this seems to be gene dependent. Alternative splicing may result in the partial or complete skipping of exons, or the partial inclusion of introns, as documented at the transcription level. This post-transcriptional process can generate multiple isoforms from a single KIR gene, which diversifies the characteristics of the encoded proteins. For example, alternative splicing could modify ligand interactions, cellular localization, signaling properties, and the number of extracellular domains of the receptor. In humans, we observed abundant splicing for KIR2DL4, and to a lesser extent in the lineage III KIR genes. All experimentally documented splice events are substantiated by in silico splicing strength predictions. To a similar extent, alternative splicing is observed in rhesus macaques, a species that shares a close evolutionary relationship with humans. Splicing profiles of Mamu-KIR1D and Mamu-KIR2DLO4 displayed a great diversity, whereas Mamu-KIR3DL2O (lineage V) is consistently spliced to generate a homolog of human KIR2DL5 (lineage I). The latter case represents an example of convergent evolution. Although just a single KIR splice event is shared between humans and macaques, the splicing mechanisms are similar, and the predicted consequences are comparable. In conclusion, alternative splicing adds an additional layer of complexity to the $K I R$ gene system in primates, and results in a wide structural and functional variety of KIR receptors and its isoforms, which may play a role in health and disease.
\end{abstract}

\title{
Introduction
}

Natural killer (NK) cells express killer-cell immunoglobulin-like receptors (KIR) that interact with major histocompatibility complex (MHC) class I molecules expressed on the cell surface of nucleated cells. Through these interactions, KIR may modulate the NK-cell activity, thereby providing regulation of the immune system in infectious diseases, pregnancy, and transplantation (1-4). KIR belong to a multigene family, which in humans comprises seventeen members that are categorized into four lineages based on structure and ligand interactions; lineage I includes $K I R 2 D L 4 / 5$, lineage II includes KIR3DL1/L2/S1, lineage III includes KIR2DL1-3/2DS1-5 and the pseudogenes, and lineage $V$ includes KIR3DL3. The KIR gene cluster is a complex entity, as is reflected by allelic polymorphism (5), gene copy number variation resulting in different haplotypic configurations (6), variegated expression $(7,8)$, and complex chromosomal recombination events (9-11). 
The KIR genes are tandemly arranged on chromosome 19q13.4, each spanning 10,000-15,000 base pairs (bp), and are separated by $1,000 \mathrm{bp}$ (12). The receptors are encoded by up to nine exons, of which the first two exons encode the leader peptide, followed by exons encoding two or three extracellular Ig-like domains (2D or 3D; exons 3-5), a stem structure (exon 6), a transmembrane region (exon 7), and a cytoplasmic tail (exons 8-9) $(13,14)$. A long cytoplasmic tail (L) contains two immunoreceptor tyrosine-based inhibitory motifs (ITIM) and characterizes inhibitory KIR. Activating KIR feature a short cytoplasmic tail (S) and a positively charged residue in the transmembrane region, which interacts with molecules that contain the immunoreceptor tyrosinebased activation motif (ITAM).

In the past, numerous KIR characterization studies were mainly performed at the genomic DNA (gDNA) level, thereby lacking information about transcription and posttranscriptional modifications (PTM) of the transcripts. Recently, next-generation sequencing (NGS) has improved and speeded up characterization of the KIR gene cluster, resulting in the identification of novel alleles, recombinant genes, and haplotypes $(9-11,15-17)$. In addition, NGS also enables the characterization of transcripts that are subjected to alternative splicing. The alternative splicing of transcripts is a prevalent form of PTM, which is observed for approximately $95 \%$ of the human multi-exon genes, and it plays a crucial role in the regulation of protein diversity and tissue-specific gene expression $(18,19)$. Normally, precursor messenger RNA (premRNA) is converted to mRNA by constitutive splicing, which involves the removal of introns and the ligation of exons by the spliceosome, which is a complex of five small nuclear RNAs (U1, U2, U4, U5, and U6), and multiple associated core proteins (20-22). To correctly identify the splice sites, a precise interplay of conserved sequence elements present on pre-mRNA (cis-acting), along with spliceosome factors, (transacting) is required. Deviation from constitutive splicing, caused by variation and mutations in the pre-mRNA sequence and/or an imbalance in the trans-acting splice factors, can result in alternative splicing. This process can be either beneficial, as a wide variety of isoforms can originate from a single gene, or detrimental, as different isoforms can be involved in the development of various diseases, such as spinal muscular atrophy and different forms of cancer (23-25). Despite its clinical relevance, at present the splicing pattern of only a few multi-exon genes has been described thoroughly $(26,27)$.

Three groups reported alternatively spliced KIR transcripts that lacked complete exons (28-32). For example, KIR2DL4 transcripts may lack exon 3 (D0 domain), suggesting the existence of a protein structure with only the D2 extracellular domain (30). Likewise, KIR2DL5 splice variants with deletions of exon 5, exons 3 and 5, and exon 7 have been reported; these might encode protein structures lacking the extracellular D2 domain, both the D0 and D2 domains, and the transmembrane region, respectively (31). The latter suggests the existence of a soluble KIR2DL5 isoform. In addition, deletions of only fragments of the Ig-like domains have been described. 
Over the past few years, the KIR gene family has also been characterized in nonhuman primate species, which provide insights into the evolution of this section of the immune system, and eventually may help to optimize and refine animal models ( 9 , 33-37). Rhesus macaques (Macaca mulatta, Mamu), for example, share a close evolutionary relationship with humans, as is reflected by similar immune responses and pathologies in many models for infectious and autoimmune diseases (38-40). Although there are certain subtle differences such as different receptor lineage expansion (lineage II and III in macaques and humans, respectively), and the absence of a haplotype $A$ and $B$ organization in macaques, the KIR cluster in macaques is highly similar to that observed in humans $(9,41)$. Within the rhesus macaque KIR family, twenty-two genes are identified, including receptors with a single extracellular domain (Mamu-KIR1D), a homolog of human KIR2DL4, and multiple KIR3D gene structures (33, 42). Rhesus macaque KIR haplotypes can contain four to fourteen genes, illustrating the extensive copy number variation $(9,33)$. As observed in humans, the macaque KIR cluster is also characterized by allelic variation, variegated expression, and chromosomal recombination.

Alternatively spliced KIR transcripts with deletions of complete or partial domainencoding exons are described for a few Mamu-KIR3D genes, identified by Sanger sequencing $(36,42,43)$. For example, the Mamu-KIR3DL20 gene, which shows sequence similarity with human lineages I and $V K I R$, is hypothesized to consistently generate Mamu-KIR2DLO5 transcripts by the skipping of exon $4(33,42,44,45)$.

Although a few KIR splice variants were already reported in different primate species, the current literature lacks a comprehensive overview of the modifications of KIR genes generated by alternative splicing, as well as an indication of its possible functional consequences. By using a Single-Molecule, Real-Time (SMRT) sequencing approach on the Pacific Bioscience's (PacBio) Sequel platform, we were able to thoroughly characterize the alternative splicing of KIR gene transcripts in both human and rhesus macaque families. The chosen high-resolution method provides insights into the segregation of alternatively spliced KIR transcripts and the potential splicing mechanisms. The data illustrated that alternative splicing adds another layer of complexity to the KIR family in both humans and rhesus macaques. Moreover, the alternatively spliced KIR gene isoforms might encode receptors having a modified structure, function, and/or expression profile, which consequently might play a custom role in health and disease. 


\section{Materials and Methods}

\section{Transcriptome datasets}

The KIR transcriptomes of 15 related humans and 30 related rhesus macaques were reported previously (9). In addition, during the course of this study the KIR transcriptomes of another three rhesus macaque families, which in total comprised 25 macaques, and one human family, comprising six individuals, samples of whom were provided by Sanquin (Amsterdam, The Netherlands), were analyzed as previously described (9). In short, total RNA was isolated from human and rhesus macaque PBMCs, and cDNA was synthesized. Primer sets were designed for human KIR2DL4 and $K I R 2 D / 3 D$, which amplified all human KIR genes except for KIR3DL3, KIR2DL5, and the pseudogenes. A KIR2DLO4-specific primer set along with two KIR2D/3D primer sets amplified macaque KIR. Tagged KIR amplicons were pooled and purified, and SMRTbell libraries were generated. Sequencing was performed on a PacBio Sequel platform using P6-C4 sequencing chemistry. Informed consent was obtained from all participants.

\section{Identification of alternative spliced KIR transcripts and splice elements}

Subsequent to PacBio sequencing, the circular consensus sequences were selected for high read quality, and were demultiplexed based on unique barcoding. Geneious Pro R10 software (46) was used to map the PacBio reads to reference databases that included all reported full-length and partial human and rhesus macaque KIR allele sequences, which were derived from the IPD-KIR database and the literature $(5,33,43$, $45,47-51$ ), to identify $100 \%$ matched reads ( $0 \%$ mismatch, maximum ambiguity $=1$, minimum mapping quality $=30,80 \%$ minimum overlap identity, minimum overlap $=$ 400). Next, the unused reads were subjected to deletion and structural variant discovery, which can align paired and unpaired reads that include structural rearrangements, deletions, and insertions, to reference sequences from the databases ( $0 \%$ mismatch, maximum ambiguity $=1$, minimum mapping quality $=30,10$ gaps per read allowed, minimum overlap $=100)$. A splice variant was confirmed when observed in two or more individuals with at least three supporting reads. For each gene for which a specific splice event was confirmed, the sequence was submitted to the ENA database and received an accession number (Suppl. Table $\mathbf{1}$ and $\mathbf{2}$ ). In addition, Sanger sequencing was used to confirm alternative splicing in transcripts of human KIR2DL5 (deletion of 294 bp), human KIR2DL4 (deletion of 104/105 bp), Mamu-KIR3DLO1 (inclusion of $170 \mathrm{bp}$ ), Mamu-KIR3DL20 (deletion of $294 \mathrm{bp}$ and $300 \mathrm{bp}$ ), and MamuKIR2DLO4 (inclusion of $245 \mathrm{bp}$ ), using primers designed in the inserted region, or at the boundary of the deleted region. The skipping of exon 4 in Mamu-KIR3DL20 transcripts was visualized by gel electrophoresis, using gene-specific primers situated at the boundary of exons $1 / 2$ and at the end of exon 5 . 


\section{KIR intron sequences}

Macaque KIR intron sequences are almost absent from the literature, except for introns in a completely sequenced rhesus macaque KIR haplotype (45). Therefore, we extracted genomic DNA (gDNA) from EDTA whole blood samples by a standard saltingout procedure, or from $\sim 15 \times 10^{6}$ PBMCs with an AllPrep RNA/DNA Mini Kit (Qiagen), in accordance with the manufacturer's instructions. We designed generic primer sets, tagged with PacBio barcodes, which amplified one or multiple introns, and flanking exons, of Mamu-KIR3DL/S, Mamu-KIR1D, and Mamu-KIR2DLO4 (Suppl. Table 3). Thermal cycling conditions were denaturation at $98^{\circ} \mathrm{C}$ for 2 min, followed by " $x$ " cycles of $98^{\circ} \mathrm{C}$ for $20 \mathrm{~s}, " x$ " ${ }^{\circ} \mathrm{C}$ for $30 \mathrm{~s}$, and $72^{\circ} \mathrm{C}$ for 2 min (number of cycles and annealing temperature are indicated in Suppl. Table $\mathbf{3}$ for each primer set). Sequencing was performed on a PacBio Sequel platform. The majority of the human KIR intron sequences were derived from the IPD-KIR database (52). Additional sequences of human $K I R$ introns 6,7 , and 8, and flanking exons, were obtained by amplification with two primer sets, using the above-mentioned thermal cycling conditions (Suppl. Table 3). The obtained intron sequences could be assigned to the corresponding KIR genes or alleles based on the flanking exon sequences.

\section{Splicing strength prediction}

Multiple prediction tools have been developed and compared to score sequence elements that are involved in splicing, such as the $3^{\prime}$ splice site (ss) region, including the polypyrimidine tract (PPT), and the $5^{\prime}$ ss (53-57). In all studies, the Maximum Entropy Modeling Scan (MaxEntScan; MES) (58), the Position Weight Matrix (PWM) via SpliceView (59), and the Human Splice Finder (HSF) (60) outperformed the other tools, and were therefore selected to predict the splicing strength of the KIR splice elements. The different prediction tools use varying nucleotide ranges to score the splicing strength of the $3^{\prime} \mathrm{ss}$, which is likely due to the degenerate nature of this motif (Suppl. Table 4). The $5^{\prime}$ ss was mainly defined by three exonic (-3) and six intronic nucleotides (+6). The output value of the tools also has different ranges, but a higher score always implies a more precise prediction (Suppl. Table 4). It should be noted that the scores are not a measure of effect sizes, and there are no thresholds that can predict whether or not a splice event will occur. The scores should only be used to facilitate a comparison between related splice sites. In addition to MES, PWM, and HSF, the Weight Matrix Model (WMM) (58) and NNSplice tool (61) were evaluated, and used when other tools failed to provide a splicing strength score. 


\section{Results}

\section{Overview of alternative splicing events in human KIR genes}

In a preceding family study, single Molecule, Real-Time (SMRT) sequencing on a Pacific Bioscience's (PacBio) Sequel platform was used to obtain KIR2DL4 and KIR2D/3D transcript profiles of fifteen related individuals (9). These transcript profiles partly consisted of reads that matched $100 \%$ to known KIR alleles. The dataset, however, also comprised a considerable number of partial sequences, and sequences that contained single nucleotide gaps. In this communication, we performed an in-depth analysis of these latter datasets, and determined that approximately $53 \%$ and $4 \%$ of the $100 \%$-matched reads (error-free reads) accounted for alternatively spliced KIR2DL4 and KIR2D/3D transcripts, respectively.

In total, 29 distinct KIR splice events were identified ( $\geq 3$ PacBio reads), of which 18 were observed in two or more related individuals (Table 1). These independently confirmed splice events involved both insertions ( 6 events) and deletions (12 events), and can be categorized into common types of splicing mechanisms, such as exon skipping, alternative $3^{\prime}$ - and 5'-splice sites (ss), and cryptic exon inclusion (Figure 1 and

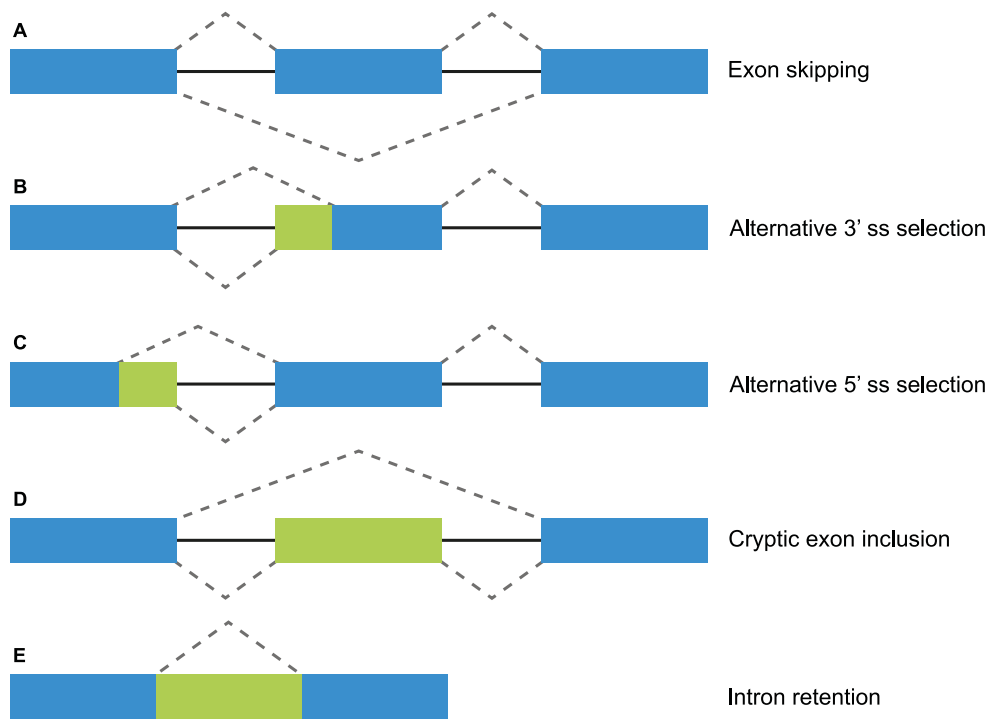

Figure 1. Different mechanisms of alternative splicing. Blue boxes indicate exonic regions. Green boxes indicate intronic sequences that are included in mature mRNA by alternative splicing. The upper dashed lines indicate constitutive splicing, whereas the lower dashed lines indicate alternative splicing. (A) Exon skipping (or 'cassette exon') is the most prevalent form of alternative splicing, which involves the complete deletion of one or multiple exons. (B, C) An alternative $3^{\prime}$ or 5' splice site (ss) can result in a partial intron retention (observed as insertion in the mRNA) or deletion. (D) Intron retention is a less common form of alternative splicing, and involves the inclusion of a complete intron. (E) Introns can include exonic sequences that can contain intact $3^{\prime}$ and $5^{\prime}$ splice sites. The incorporation of these cryptic exons is prohibited during constitutive splicing, but they can be included into the mature mRNA by alternative splicing. 


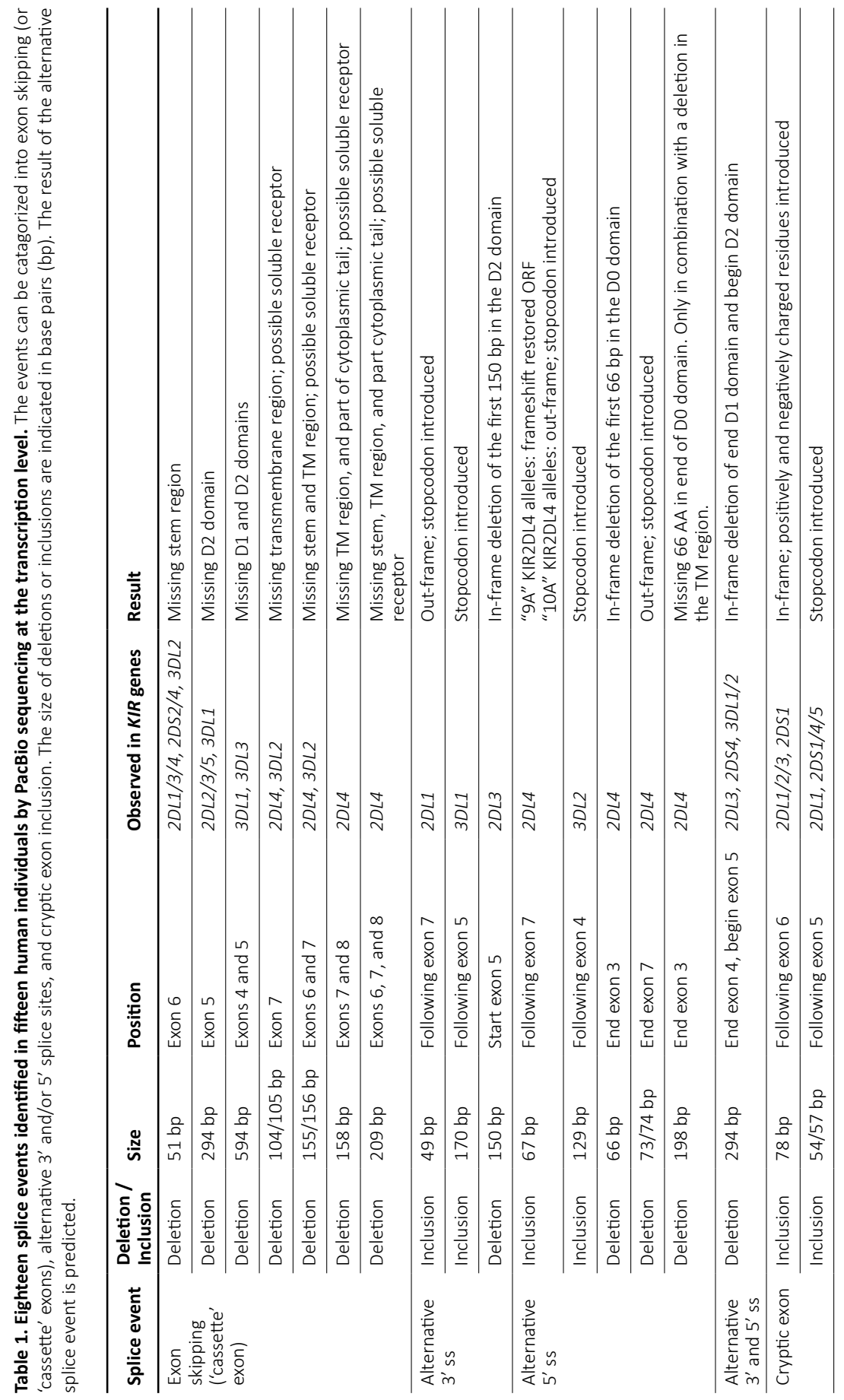


Table 1). In figure $\mathbf{2 A}$, a schematic overview is provided of the confirmed splice events summarized in Table 1. The excision of exon 6, which encodes the stem region, represented the most frequently observed splice event, and was identified in alleles of six different KIR genes (Table 1). Other commonly observed splice events were the deletion of exon 5 (D2 domain), a deletion of 294 bp mediated by an alternative $5^{\prime}$ ss at the end of exon 4 and an alternative $3^{\prime}$ ss at the beginning of exon 5 , an insertion of $54 / 57$ bp following exon 5, and an insertion of $78 \mathrm{bp}$ subsequent to exon 6 . The remaining splice events were specific for one or two KIR genes. In transcripts of KIR2DL1, KIR2DL3, KIR2DL4, KIR3DL1, and KIR3DL2, at least four different splice events were observed, resulting in a diverse range of isoforms for these genes. The most diverse alternative splicing profile was observed for KIR2DL4, for which eight different splice events were identified. Most of these events were KIR2DL4-specific, including a frequently observed insertion of $67 \mathrm{bp}$ subsequent to exon 7, and a deletion of $66 \mathrm{bp}$ in exon 3. For transcripts encoded by activating $K I R$ genes less alternative splicing events were observed, which might be explained by the lower frequency of these genes in the individuals studied. Similar splice events were observed in an additional human family comprising six individuals, confirming the obtained splice profiles, and suggesting that the data provides a comprehensive overview of alternative splicing in human KIR.

\section{In silico prediction of cis-acting splicing elements}

Constitutive and alternative splicing of pre-mRNA is regulated by trans-acting factors (small nuclear RNAs, spliceosome core proteins), and their cognate nucleotide sequence cis-elements near the intron-exon boundaries $(54,62)$. Essential splicing ciselements are the $3^{\prime}$ splice site (ss), the $5^{\prime}$ ss, the branch point sequence (BPS), and the polypyrimidine tract (PPT) (Figure 3). Additional enhancer and silencer elements can be identified in the exons (Exon Splicing Enhancer, ESE; Exon Splicing Silencer, ESS) and introns (Intron Splicing Enhancer, ISE; Intron Splicing Silencer, ISS). These regulatory splicing sequences are degenerate, and the consensus sequences can only be loosely followed (63). Although software tools are available to predict and score the splicing strength of all different cis-elements (54), we mainly focused on the better modeled prediction of the splice site elements ( $3^{\prime}$ - and $5^{\prime}$ SS, BPS, and PPT). In the following sections, different observed events (Table 1, Figure 2A) are substantiated by the identified splice sites, and by their corresponding in silico-predicted splicing strength scores, per alternative splicing mechanism (Figure 1).

\section{Exon skipping in human KIR transcripts}

The skipping of one or multiple exons was the most frequently observed alternative splicing mechanism in the KIR transcriptomes of the human family studied (Figure 1A, Table 1, and suppl. Table 1). The skipping of exon 7, which encodes the transmembrane region, was observed in KIR2DL4 and KIR3DL2 transcripts, and might be explained by variation in the splicing cis-elements (Figure 4). In all KIR genes, identical BPS and 3' sS sequences were identified in intron 6 preceding exon 7, and were in agreement with 

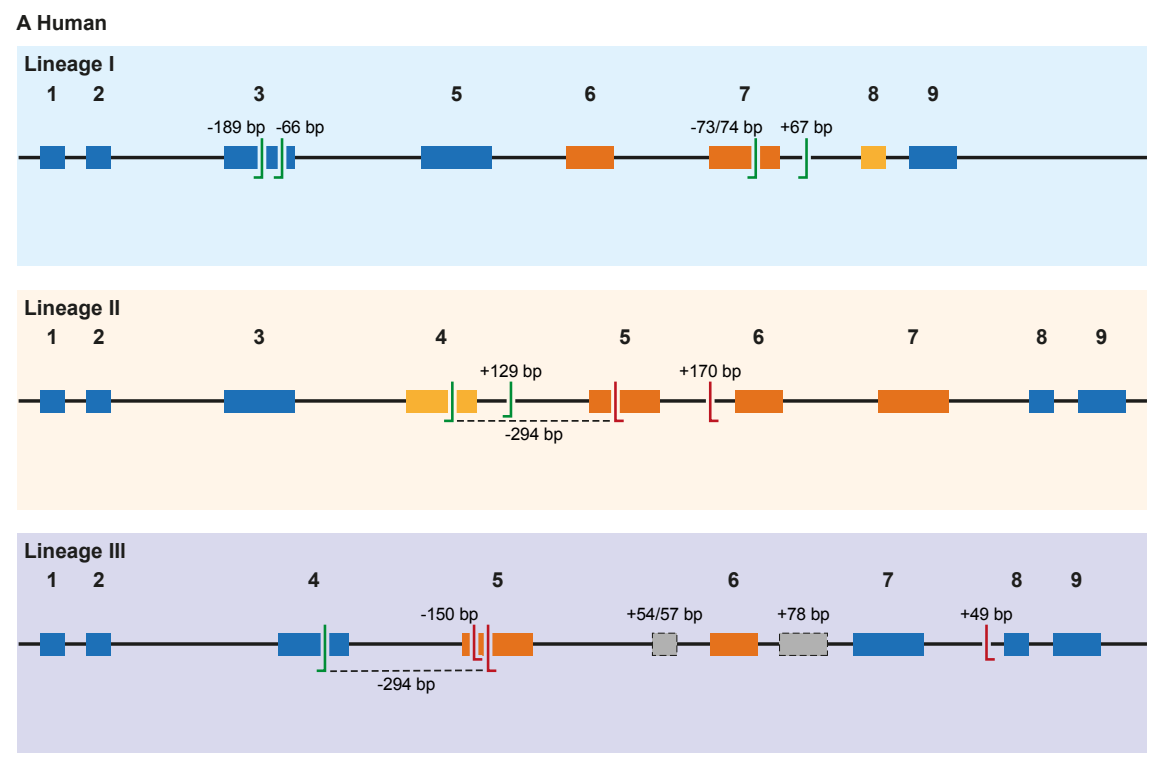

\section{B Macaque}

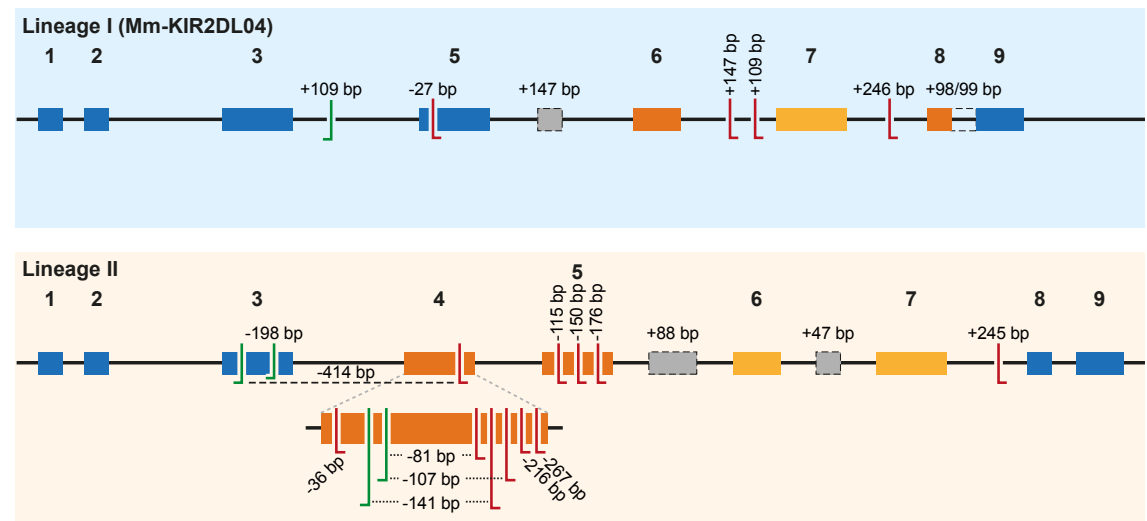

Lineage V (Mm-KIR3DL20)

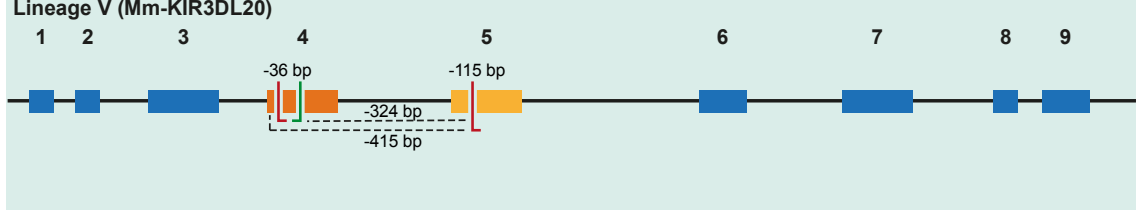


Figure 2. Overview of alternative splice sites in human and rhesus macaque KIR. A schematic representation of the different splice events observed in human (A) and rhesus macaque (B) KIR transcripts categorized by gene lineage. The splice events illustrated correspond to the splice events summarized in tables 1 and 2 , and are indicated with the size of the inclusion (+) or deletion (-) in base pairs (bp), or by color-coding. The black line indicates the introns, whereas colored boxes represent the exons. Exons that are subjected to exon skipping are illustrated with dark orange boxes, and the exons that are only skipped in combination with one or more exons are indicated in light orange boxes. Exons that are not subjected to exon skipping are colored blue. The actual splice sites, which map to the exon/intron boundaries, have not been indicated. Alternative $3^{\prime}$ splice sites (ss) are indicated with green left-directed hooks, whereas alternative $5^{\prime}$ ss are indicated with red right-directed hooks. An alternative splice site always pair with the adjunct complement actual $3^{\prime}$ or $5^{\prime}$ splice site, except splice events that are mediated by a set of $3^{\prime}$ and $5^{\prime}$ alternative ss, which are marked with a dashed line. Cryptic exons are illustrated as grey boxes with a dashed line, and the (alternative) splice sites of these cryptic exons are not explicitly indicated. The intron retention event observed in rhesus macaque KIR2DL04 (lineage I) is indicated with a dashed line between exons 8 and 9. Exon 4 in rhesus macaque lineage II KIR genes is enlarged to more precisely illustrate the high number of alternative splice events observed.

the consensus sequences YUNAY and NYAG/G (Figures $\mathbf{3}$ and $\mathbf{4}$; / marks actual splice site), respectively (64). Compared to the 5 ' ss sequence of lineage III KIR genes (MES: 9.72; HSF: 88.47; PWM: 87), a single nucleotide variation (T/C) was observed in the KIR2DL4 5' ss sequence of exon 7, and this resulted in a lower in silico-predicted splicing strength score (MES: 7.31; HSF: 86.29; PWM: 84) (Figure 4). Also for KIR2DL5 and KIR3DL2, a decreased splicing strength score was predicted for the $5^{\prime}$ ss sequence of exon 7 (MES: 9.35; HSF: 83.61; PWM: 84), and these genes are discriminated from other KIR genes by a single nucleotide as well (A/G). Furthermore, the PPT of exon 7 in KIR2DL1 and KIR2DL2 contained a single adenine substitution as compared to the remaining lineage III KIR genes, but despite the predicted lowered 3' ss splicing strength score, the skipping of exon 7 was not observed for the corresponding transcripts. The

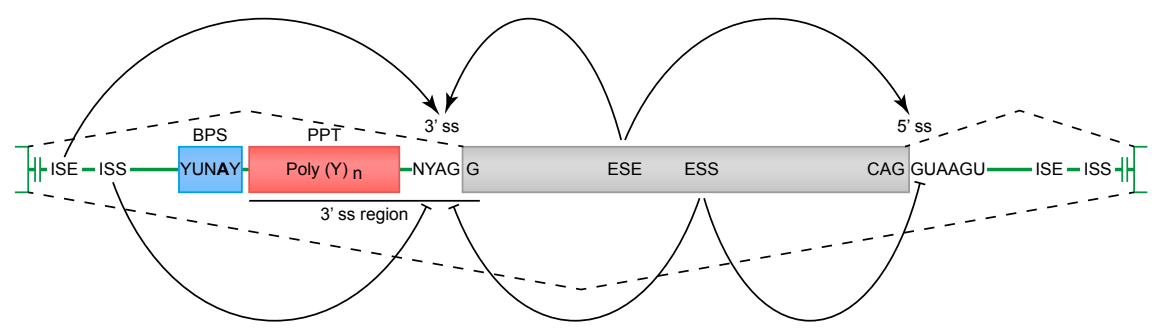

Figure 3. Cis-acting motifs that mediate constitutive and alternative splicing. The boundaries of exons (grey box) and introns (green line) are marked by splice sites (ss). At the $3^{\prime}$ ss, the end of the intron is characterized by an adenine and guanine (AG), and forms the basis of the $3^{\prime}$ ss motif. At the $5^{\prime}$ ss, the start of the intron is marked by a guanine and thymine (GT), and forms the basis of the $5^{\prime}$ ss motif ( -3 bp in exon, and +6 bp in intron). Prior to the $3^{\prime}$ ss, a branch point sequence (BPS) and polypyrimidine tract (PPT) can be identified, and these elements are involved in spliceosome binding and intron exclusion. The $3^{\prime}$ splice site and PPT together are referred to as the $3^{\prime}$ region (-20 bp in intron, and $+3 \mathrm{bp}$ in exon), and can be used to predict the splicing strength. In addition to the splice site motifs, enhancer and silencer motifs can be identified in the exons (Exon Splicing Enhancer, ESE; Exon Splicing Silencer, ESS) and introns (Intron Splicing Enhancer, ISE; Intron Splicing Silencer, ISS), and can stimulate or inhibit splicing of an exon. 


\begin{tabular}{|c|c|c|c|c|c|}
\hline \multirow{3}{*}{$\begin{array}{l}\text { KIR2DL3+ } \\
\text { KIR2DS1-5 } \\
\end{array}$} & \multicolumn{2}{|r|}{ MES: 10.88; HSF: 96.78; PWM: 93} & & \multicolumn{2}{|c|}{ MES: 9.72; HSF: $88.47 ;$ PWM: 87} \\
\hline & \multicolumn{2}{|r|}{ 3' ss region } & & \multicolumn{2}{|c|}{ 5'ss } \\
\hline & BPS & TGTCTCCTCTTCTT-CCAG G & Exon 7 & AAA & GTAAGT \\
\hline \multirow[b]{3}{*}{ KIR2DL1/2 } & \multicolumn{2}{|r|}{ MES: 10.37; HSF: 94.86; PWM: 90} & & \multicolumn{2}{|c|}{ MES: 9.72; HSF: 88.47; PWM: 87} \\
\hline & \multicolumn{2}{|r|}{ 3' ss region } & & \\
\hline & BPS & CCAG $\mathrm{G}$ & Exon 7 & --- & 5 'ss \\
\hline \multirow[b]{3}{*}{ KIR2DL4 } & \multicolumn{2}{|r|}{ MES: 9.72; HSF: 95.33; PWM: 90} & & \multicolumn{2}{|c|}{ MES: $7.31 ;$ HSF: $86.29 ;$ PWM: 84} \\
\hline & \multicolumn{2}{|r|}{ 3' ss region } & & \\
\hline & BPS & АT----A----C - CCAG G & Exon 7 & --- & 5' ss \\
\hline & & MES: $8.90 ;$ HSF: $92.59 ;$ PWM: 89 & & MES: $9.35 ; \mathrm{HS}$ & F: 83.61; PWM: 84 \\
\hline & & 3'ss region & & & 5'ss \\
\hline \multirow[t]{3}{*}{ KIR2DL5 } & BPS & AT- $-\mathrm{C}-\mathrm{AC}----\mathrm{CC}-\mathrm{CCAG} \mathrm{G}$ & Exon 7 & $-G-$ & \\
\hline & & MES: 9.97; HSF: 95.33; PWM: 90 & & \multicolumn{2}{|c|}{ MES: 9.35 ; HSF: 83.61 ; PWM: 84} \\
\hline & \multicolumn{2}{|r|}{3 'ss region } & & \multicolumn{2}{|r|}{$\overline{5 ' s s}$} \\
\hline KIR3DL2 & BPS & AT- $-\mathrm{C}-\mathrm{A}-----\mathrm{C}--\mathrm{CCAG}$ G & Exon 7 & $-G-$ & \\
\hline
\end{tabular}

Figure 4. Suboptimal splice sites might mediate the skipping of exon 7 in KIR2DL4, KIR2DL5, and KIR3DL2 transcripts. Exon 7 is indicated in grey boxes, whereas the BPS and PPT elements are indicated in blue and red boxes, respectively. The $3^{\prime}$ ss region ( 3 ' ss and PPT) and the 5' ss sequences of lineage III KIR genes, except for $K I R 2 D L 1 / 2$, are used as consensus sequences. Dashes (-) indicate sequence identity with the consensus sequence. The splicing strength scores of the $3^{\prime}$ and $5^{\prime}$ splice sites are provided. The BPS and $3^{\prime}$ splice sites of exon 7 were identical, but variation was observed in the PPT and 5' splice site.

PPT of exon 7 of KIR2DL4, KIR2DL5, and KIR3DL2, however, varied from the lineage III $K I R$ genes at four to seven nucleotide positions. This variation included the presence of two adenines that interrupted the guanine- and thymine-rich tract, and although a long continuous PPT is not required for splicing, it does appear to increase the splicing efficiency $(65,66)$. Indeed, a decreased splicing strength score of the $3^{\prime}$ ss region (3' ss + PPT) of exon 7 in KIR2DL4, KIR2DL5, and KIR3DL2 was predicted (Figure 4). Thus, the observed skipping of exon 7 in KIR2DL4 and KIR3DL2 transcripts might be explained by deviations in the 5' ss and the PPT together, and suggest the existence of soluble isoforms of these receptors. In addition, the absence of exon 7 in KIR2DL4 molecules results in the loss of their activating signaling potential, which is facilitated by a positive residue in the transmembrane region. Based on the data derived from the prediction tools, the skipping of exon 7 could be expected in KIR2DL5 transcripts as well, and was indeed reported previously (31). However, despite the presence of the KIR2DL5 gene in some individuals, we did not identify the event in the human family studied.

Other exon skipping events involved the complex of exons 4 and 5, exon 5 only, and exon 6, respectively, encoding the extracellular domains and the stem region (Figure 2A). In particular, the skipping of exon 6 was frequently identified, and observed 
in KIR genes of lineages I, II, and III, suggesting the presence of conserved suboptimal cis-elements. However, between the different KIR genes, extensive nucleotide variation was observed in the BPS, PPT, and $5^{\prime}$ ss of exon 6 , which resulted in a variety of predicted splicing strength scores, implying that conserved suboptimal splice sites did not mediate the splice event. In addition to less efficient splice sites, skipped exons are often characterized by longer flanking introns that can obstruct exon recognition, or that contain splice enhancer and silencer motifs (62). The two largest introns of the KIR gene are those flanking exon 6 , and might mediate exon skipping. Phylogenetic analysis of introns 5 and 6 illustrated, however, that the introns do not have a close evolutionary relationship across the different $K I R$ lineages. Despite lineage variation in the introns, ISE and ISS motifs, or elements that induce secondary intron structures, might be conserved between these introns, but these elements are hard to predict using the available in silico models.

\section{Alternative splice sites in human KIR}

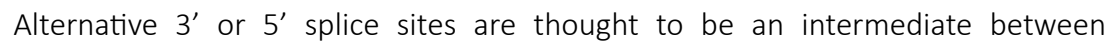
constitutively spliced and skipped exons, and can introduce in- and out-frame deletions and insertions in transcripts (Figures 1B, 1C, and 2A) (62). An example of an alternative splice event, caused by an alternative $3^{\prime}$ ss, is the retention of 170 bp of intron 5, which was observed in KIR3DL1 transcripts (Table 1). This partial intron retention introduced a premature stop codon subsequent to exon 5 , resulting in a transcript that encodes only the extracellular domains, and could be explained by the presence of an additional 3' splice site upstream of the actual splice site (Figure 5A). However, according to the in silico models, the splicing strength of the alternative 3' ss region (MES: 4.72; HSF: 77.69; PWM: 81) is remarkably lower compared to the actual splice site (MES: 11.54; HSF: 80.64; PWM: 86), which might indicate that this splice event is not common. Additionally, a BPS prior to the alternative $3^{\prime}$ ss that matches the consensus sequence was not observed. The low number of PacBio reads ( $\leq 6$ reads) for this KIR3DL1 splice variant might already be indicative that this splice event, although observed in three individuals, is not favorable over constitutive splicing. Even more, the introduction of a premature stopcodon might be indicative for the degradation of the alternatively spliced transcript by the nonsense-mediated decay. Nonetheless, the presence of this splice variant was confirmed by Sanger sequencing, and might still have functional relevance in certain NK cell subsets that are resident in specific tissues.

A partial deletion at the end of exon 7 was observed in KIR2DL4 transcripts, and was mediated by an alternative $5^{\prime}$ splice site (Figure 5B). The end of exon 7 in KIR2DL4 is marked by a poly-adenine sequence that can be nine (9A) or ten (10A) nucleotides long (30). The "9A" KIR2DL4 alleles have a premature stopcodon subsequent to exon 7, suggesting the absence of a cytoplasmic tail, and thereby the loss of their inhibitory potential (Figure 5B). The " $10 A^{\prime \prime}$ KIR2DL4 transcripts encode a complete receptor, including a cytoplasmic tail with a single ITIM. Deletions of 73 and $74 \mathrm{bp}$ at the end of exon 7 were observed in transcripts of " $9 A$ " and " $10 A$ " KIR2DL4 alleles, respectively (Figure 5B, Table 1). These deletions were mediated by an alternative $5^{\prime}$ ss that is 
A

\begin{tabular}{|l|l|l|l|l|l|l|l|l|}
1 & 2 & 3 & 4 & 5 & 6 & 7 & 8 & 9
\end{tabular}

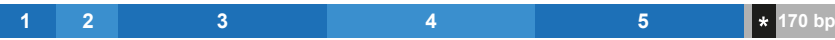

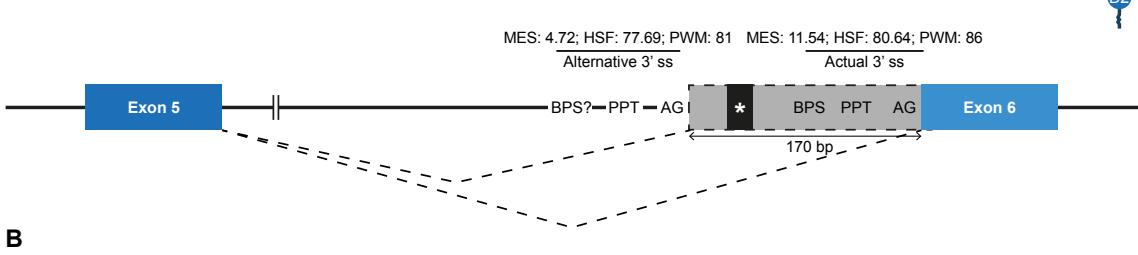

9A KIR2DL4

$1 \quad 2$

3

5

6

7

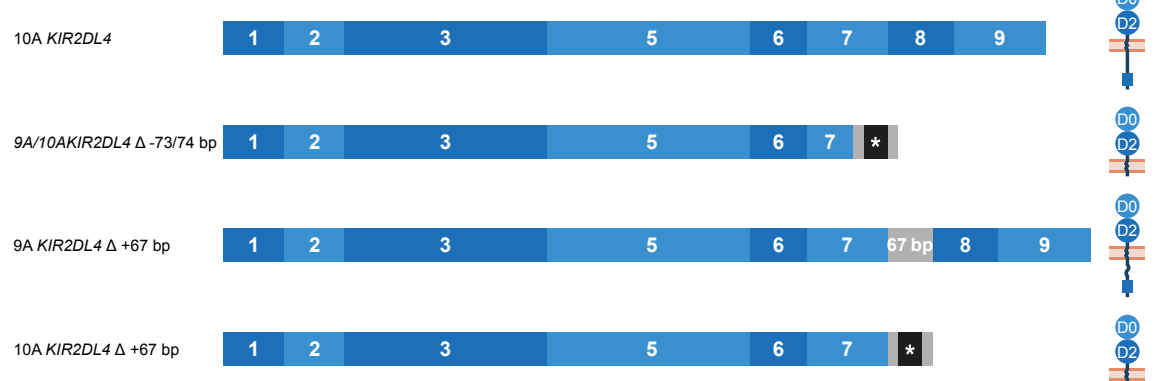

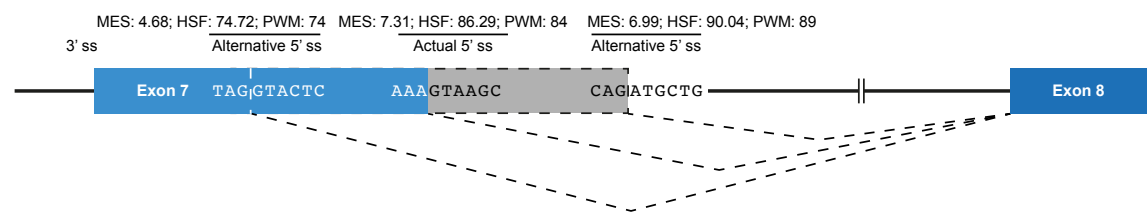

Figure 5. Alternative splice sites mediate deletions and insertions at the transcription level. The observed transcripts are illustrated, in which exons are indicated by blue boxes, and corresponding protein structures are schematically depicted adjacent to the transcript. Intron inclusions are indicated in grey boxes. Dashed lines indicate the potential splice events, and predicted splicing strength scores are provided for actual and alternative splice sites. Stopcodons are indicated by black boxes with an asterisk (*). (A) The constitutive splicing of human KIR3DL1 results in a transcript including nine exons, and encodes a KIR3DL molecule. Alternative splicing, mediated by an alternative $3^{\prime}$ splice site located 170 bp downstream of the actual splice site, results in a transcript encoding only three extracellular domains. "BPS?" refers to the potential lacking of a BPS for the alternative 3 ' ss. (B) The constitutive splicing of human "9A" and "10A" KIR2DL4 alleles results in membrane-bound molecules containing two extracellular domains, or molecules that contain two extracellular domains and a cytoplasmic tail including a single ITIM, respectively. An alternative $5^{\prime}$ ss located in exon 7

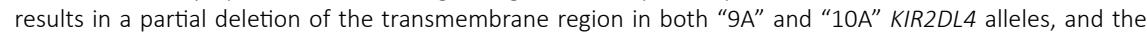
introduction of a stopcodon. A second alternative 5 ' ss located in intron 7 results in a partial intron inclusion of $67 \mathrm{bp}$. In "9A" KIR2DL4 alleles, this inclusion restores the open reading frame, and these isoforms probably express an inhibitory cytoplasmic tail. In contrast, the same splice event in "10A" KIR2DL4 alleles results in a frame-shift that introduces a stopcodon subsequent to exon 7. 
located within exon 7, and caused a frameshift that introduced a premature stop codon, suggesting a soluble KIR2D molecule. The in silico models predicted that the splicing strength score of the actual 5' ss is higher (MES: 7.31; HSF: 86.29; PWM: 84) than the splicing strength score of the alternative $5^{\prime}$ ss located in exon 7 (MES: 4.68; HSF: 74.72; PWM: 74), suggesting that constitutive splicing would be more prevalent. In addition, another alternative $5^{\prime}$ ss was observed in intron 7 of KIR2DL4, which resulted in a partial intron inclusion of 67 bp subsequent to exon 7. This alternative $5^{\prime}$ ss scored a higher predicted splicing strength (MES: 6.99; HSF: 90.04; PWM: 89) then the alternative $5^{\prime}$ ss located in exon 7 , and even scored higher compared to the actual 5 ' ss according to the HSF and PWM models. This might indicate that the inclusion of 67 bp subsequent to exon 7 in KIR2DL4 transcripts is a prevalent splicing event, which is also supported by high PacBio read counts observed for this splice variant (an average of 115 PacBio reads per individual). In the "10A" KIR2DL4 transcripts, the partial intron inclusion mediated by the alternative $5^{\prime}$ ss in intron 7 caused a frameshift that introduced a stopcodon subsequent to exon 7, and they thereby lack the cytoplasmic tail that includes an ITIM. In contrast, in the "9A" KIR2DL4 alleles, which normally encode a truncated receptor, the open reading frame (ORF) was restored by the partial intron inclusion, resulting in transcripts that encode a KIR protein including a cytoplasmic tail. These examples suggest that alternative splicing might regulate whether the KIR2DL4 receptors contain a cytoplasmic tail, and thereby maintain their inhibitory function, or not.

\section{"Cryptic" exons in human KIR}

Some potential exons - referred to as cryptic exons - are located within intronic regions, and are normally not spliced into mature mRNA by constitutive splicing (Figure 1E); this could be due to intrinsic defects, the presence of splice silencer elements, or the formation of inhibiting RNA secondary structures (67). Nonetheless, alternative splicing can mediate the inclusion of cryptic exons in the transcript, as is previously described for KIR2DL1, and this might play a role in health and disease (6770). In the family studied, multiple alternative splice events that introduced a cryptic exon were identified. For example, an inclusion of $78 \mathrm{bp}$ that originated from intron 6 was observed in transcripts of KIR2DL1, KIR2DL2, KIR2DL3, and KIR2DS1 (Figure 6A). The extended transcripts remained in-frame, and the 26 introduced amino acids (cryptic exon) between the stem and transmembrane region included positively and negatively charged residues. The stretch of amino acids was found to be highly conserved in the four KIR gene products mentioned above, with only one single nucleotide variation present in the alleles studied. In all other KIR genes, except for KIR2DL5 and KIR3DL3, the cryptic exon could be identified at the same position in intron 6 ( 1426 bp 3' of exon 6, 2755 bp $5^{\prime}$ of exon 7), but sequence variability was observed, varying from five to fifteen nucleotides, as compared to the abovementioned conserved sequence. This variation might involve ESE and ESS motifs, which could inhibit cryptic exon inclusion. In KIR genes that contained the cryptic exon or the variant cryptic exon, a BPS, PPT, and 3' ss were identified, and similar 3' ss splicing 
A

\begin{tabular}{|l|l|l|l|l|l|l|l|}
\hline 1 & 2 & 4 & 5 & 6 & 7 & 8 & 9 \\
\hline
\end{tabular}

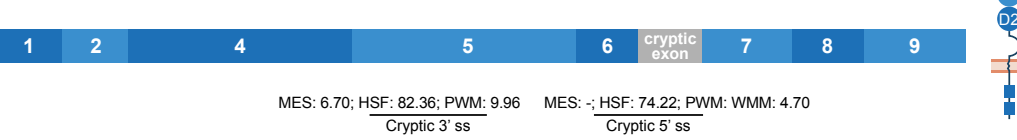

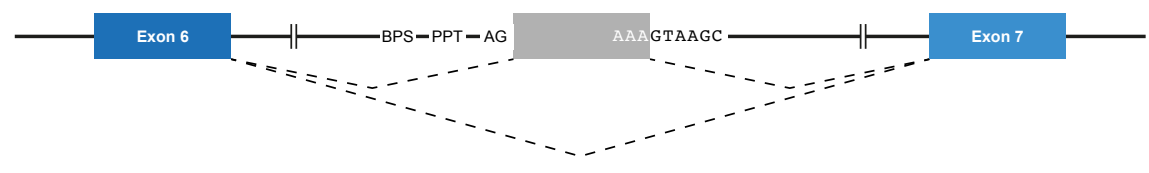

B

1

2

4

5

6 7

8 9

$\sum_{1}^{02}$

\begin{tabular}{lllll|l}
1 & 2 & 4 & 5 & $*$
\end{tabular}

D2

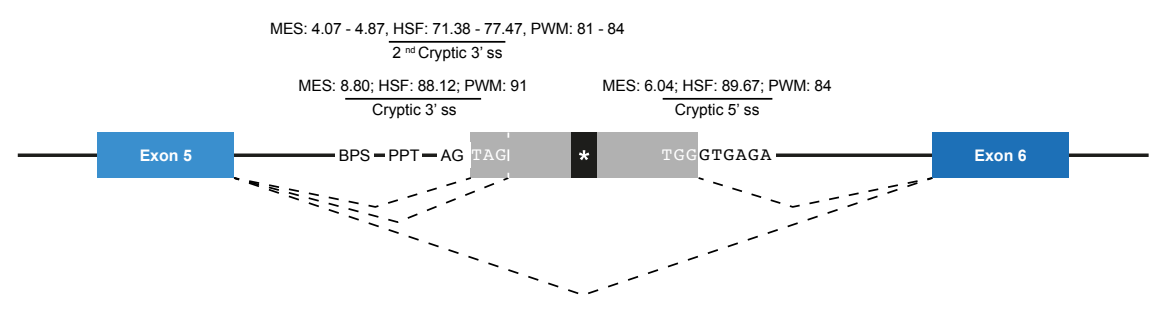

Figure 6. Inclusion of cryptic exons by alternative splicing. The observed transcripts are illustrated, in which exons are indicated by blue boxes, and corresponding protein structures are schematically depicted adjacent to the transcript. Cryptic exons are indicated in grey boxes. Dashed lines indicate the potential splice events, and predicted splicing strength scores are provided for cryptic splice sites. Stopcodons are indicated by black boxes with an asterisk $(*)$. (A) In transcripts of KIR2DL1-3/2DS1, a cryptic exon of 78 bp was observed that originated from intron 6 . This inclusion extends the region between the stem and transmembrane region by 26 amino acids, including positively and negatively charged residues. (B) In transcripts of KIR2DL1 and KIR2DS5, a cryptic exon of 57 bp was observed, which originated from intron 5 . Three bp upstream, a second cryptic 3' ss was identified, which explained the cryptic exon inclusion of 54 bp in transcripts of KIR2DS1 and KIR2DS4. At the gDNA level, the presence of one or both of these cryptic exons was also identified in other lineage III KIR genes and KIR2DL5. 
strength scores were predicted (data not shown). The $5^{\prime}$ sS of KIR2DL1-3 and KIR2DS1 could be distinguished from the $5^{\prime}$ ss of other KIR genes by a substitution of a cytosine with an adenine, which resulted in a higher predicted $5^{\prime}$ ss splicing strength score in KIR2DL1-3 and KIR2DS1 (MES:-; HSF: 74.22; PWM:-; WMM: 4.70) compared to the KIR genes that had a cytosine in the 5' ss (MES:-; HSF: 65.41; PWM:-; WMM: 1.71). Thus, this mutation might contribute to the inclusion of the cryptic exon at the transcription level. Furthermore, phylogenetic analysis illustrated that intron 6 of each KIR gene clustered separately, but that the evolutionary distance of KIR2DL1, KIR2DL2, KIR2DL3, and KIR2DS1 was small compared to the other KIR genes. Although hard to predict, the variation in intron 6 sequences might involve ISE and ISS motifs that, in combination with the cryptic exon variation and 5' ss mutation, contribute to the inclusion of the cryptic exon.

Another example of a cryptic exon inclusion is the insertion of 57 bp that was observed in transcripts of KIR2DL1 and KIR2DS5 (Figure 6B and Table 1). This cryptic exon originated from intron 5 ( $\sim 37 \mathrm{bp} \mathrm{3}$ ' of exon 5, $2259 \mathrm{bp} \mathrm{5}$ ' of exon 6), and introduced a stopcodon subsequent to exon 5 , resulting in transcripts encoding only the D1 and D2 domains. In all KIR genes, this cryptic exon could be identified at the same position in intron 5, with variation up to nine nucleotides. However, only in four genes (KIR2DL1, KIR2DS1, KIR2DS3, and KIR2DS5) does the cryptic exon have an intact 3' ss region (MES: 8.80, HSF: 88.12, PWM: 91), whereas the other KIR genes are missing a $3^{\prime}$ 'ss at this position. Three nucleotides upstream, however, another 3' ss could be identified in all lineage III KIR genes as well as in KIR2DL5 (MES: 4.07-4.87, HSF: 71.3877.47, PWM: 81-84), which could result in the inclusion of 54 bp subsequent to exon 5 , as was observed in transcripts of KIR2DS1 and KIR2DS4 (Table 1). Since the predicted splicing strength score is lower in the second cryptic 3' ss, a cryptic exon of 57 bp might be more prevalent then a 54 bp inclusion for genes that have both cryptic 3 ' ss, but quantitative techniques are required to confirm this. The predicted splicing strength of the $5^{\prime}$ ss is similar in all genes (MES: 6.04, HSF: 89.67, PWM: 84), except for KIR3DL2, in which a $5^{\prime}$ ss was not identified. Although cryptic exon inclusion events (54 or 57 bp) were only observed in four KIR genes in the human family studied (Table 1), these observations suggest that the cryptic exon of $57 \mathrm{bp}$ can be expected in transcripts of KIR2DL1, KIR2DS1, KIR2DS3, and KIR2DS5, whereas an identical cryptic exon of $54 \mathrm{bp}$ might be observed in transcripts of all lineage III KIR genes and KIR2DL5.

\section{Overview of alternative splicing in rhesus macaque KIR transcripts}

In addition to the splicing profiles of human $K I R$, we also analyzed alternative splicing of KIR transcripts in rhesus macaques. From a preceding family-based study, macaque KIR transcriptome profiles were obtained, which consisted of $100 \%$ matched Mamu-KIR sequences (20-45\%), partial sequences, and sequences that contained a single nucleotide gap (9). In-depth analysis demonstrated that approximately $24 \%$ and $13 \%$ of the $100 \%$-matched Mamu-KIR3DL/S and -KIR2DLO4 reads (error-free reads) accounted for alternatively spliced transcripts, respectively. In total, 48 different alternative splice events were identified ( $\geq 3$ PacBio reads), of which 29 were confirmed 
in two or more rhesus macaques (Table 2, Figure 2B, and suppl. Table 2). To verify whether we had obtained a complete overview of the alternative KIR splicing profiles, the PacBio read coverage of some previously typed macaque transcriptomes was increased by pooling samples of three instead of twelve rhesus macaques in a single PacBio Sequel sequencing run. This resulted in an average of 40.000 PacBio reads per rhesus macaque, which is approximately four times the number of reads we obtained per macaque from the previous study. Three additional splice events were identified ( $\geq$ 10 PacBio reads, or confirmed in two macaques; Table 3), and although a few splice events may have been missed, this indicated that the coverage of the formerly obtained KIR transcriptomes is sufficient to provide a fairly complete overview of the alternative splicing profiles. Furthermore, three additional families, which in total comprised 25 rhesus macaques, were sequenced for their KIR transcriptome, in accordance with the previously described protocol (9). The alternative splicing profiles of the KIR transcripts in these families revealed only one novel splice event (deletion of $112 \mathrm{bp}$ in exons 4 and 5), and confirmed twenty-four splice events that were already present in the alternative KIR splicing profiles of the formerly studied family. Moreover, three events that were previously identified in a single macaque (Table 3, events in italic) could be confirmed by analyses of the three additional families ( $\geq 3$ PacBio reads, in two or more macaques). This illustrated that most, but not all, KIR splice events are shared between macaque families.

\section{Common alternative splicing events in rhesus macaque KIR}

As in human KIR, all independently confirmed splice events observed in rhesus macaque KIR could be categorized into common alternative splicing mechanisms (Figure 1), which are listed in Table $\mathbf{2}$ and schematically illustrated in Figure 2B. Splice events that involved Mamu-KIR1D are not included in this table and schematic figure, and will be discussed separately in the following section. The deletion of the first $36 \mathrm{bp}$ of exon 4 was the most frequently observed alternative splice event, and is mediated by a conserved alternative $3^{\prime}$ ss that is present in most Mamu-KIR1D/3D alleles (Table 2). The predicted splicing strength score of the alternative 3' SS (MES: 8.2410.27, HSF: 90.11-90.53, PWM: 88-92) was higher (HSF and PWM), or similar (MES), compared to the predicted score of the actual 3' SS (MES: 8.25-10.20, HSF: 86.6188.52, PWM: 84-87). Transcripts with this in-frame deletion lacked 12 amino acids at the start of the D1 domain, including three positively charged residues that might be involved in protein folding or ligand binding. In exon 4 of most Mamu-KIR3D genes, two other alternative $3^{\prime}$ ss could be identified, resulting in transcripts with in-frame deletions of 216 bp and 267 bp (Figure 2B and Table 2). Likewise, four alternative 3'ss 


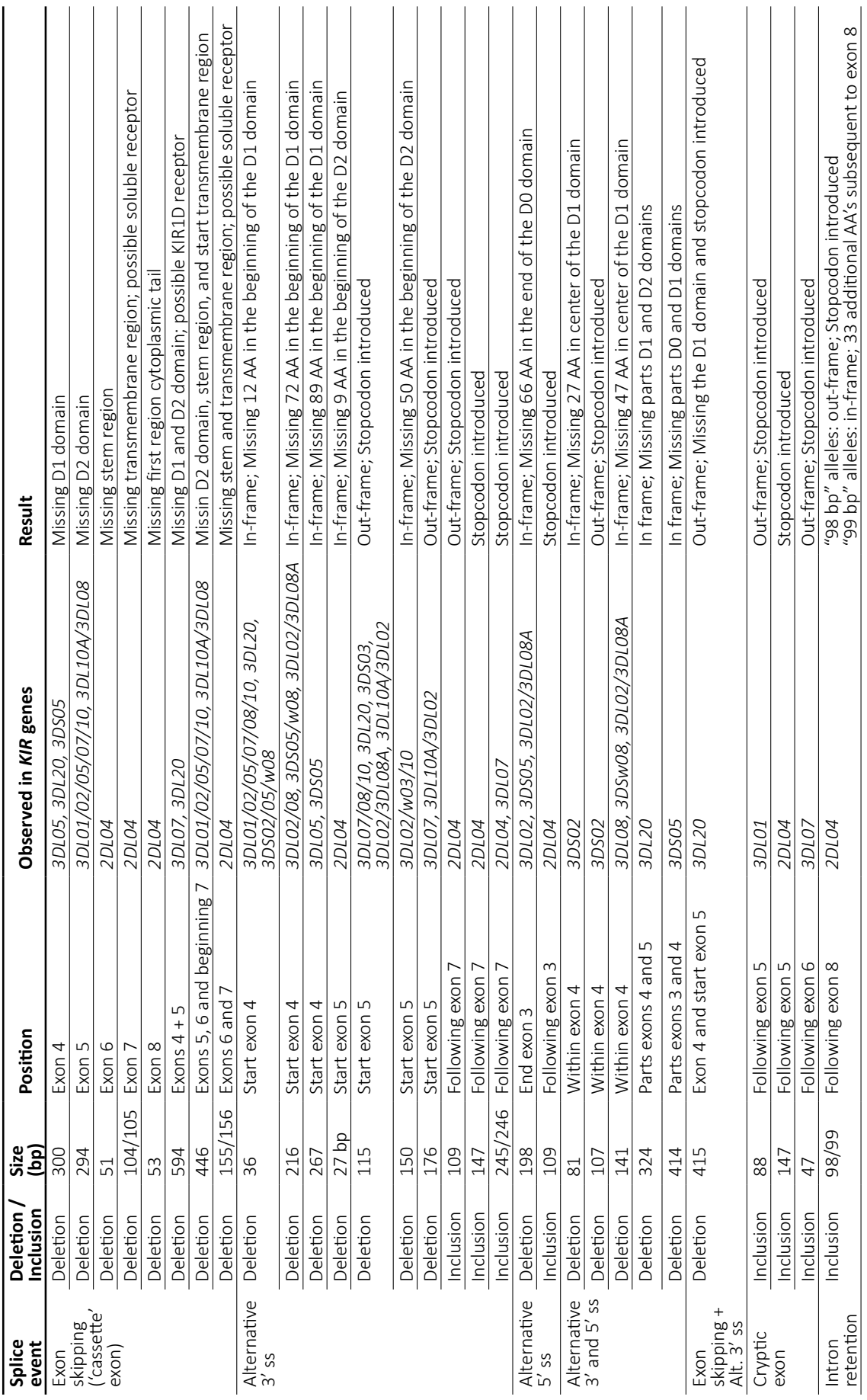


could be identified in exon 5 of most Mamu-KIR3D genes, of which the one that mediated an out-frame deletion of $115 \mathrm{bp}$ was most frequently observed. Other common alternative splice events observed in rhesus macaque KIR involved the skipping of exon 5 and the deletion of 446 bp (exons 5, 6, and 7). Mamu-KIR1D, -KIR3DL20, and -KIR2DLO4 displayed a remarkable alternative splicing profile, and will be discussed in more detail in the next sections.

\section{Extensive alternative splicing in Mamu-KIR1D}

Mamu-KIR1D, which is the only lineage III KIR gene in rhesus macaques, was identified in approximately $25-30 \%$ of the defined Mamu-KIR haplotypes, and, so far, only three different alleles are reported, suggesting a high level of conservation at the exon level (9). Hershberger and colleagues described nine different splice variants using Sanger sequencing (43). In our KIR transcriptome profiles obtained by PacBio sequencing, we identified a complex array of nineteen different alternatively spliced Mamu-KIR1D transcripts that originated from a single allele (Mamu-KIR1D*002) (Figure 7A). Up to fifteen different Mamu-KIR1D isoforms could be identified in a single individual ( $\geq 3$ PacBio reads), which illustrates extensive alternative splicing. These splice variants could be explained by exon skipping, alternative $3^{\prime}$ - and 5' ss, and cryptic exons. At the genomic DNA (gDNA) level, three domain-encoding exons could be identified, but only exon 4 (D1 domain) was present in all transcribed Mamu-KIR1D isoforms. On the basis of gDNA analysis, it was revealed that exon 3 of Mamu-KIR1D contained a 5 bp deletion (71), and was constitutively skipped, similar to what is observed in human lineage III KIR genes. However, an intact BPS, PPT, 3' sS (MES: 6.62, HSF: 86.67, PWM: 85), and 5' SS (MES: 7.41, HSF: 92.64, PWM: 86) could be identified for exon 3 of Mamu-KIR1D, which may suggest that another mechanism plays a role in the constitutive skipping of this exon. An explanation might be the absence of $33 \mathrm{bp}$ in intron 2, which characterizes all lineage III KIR genes in both humans and macaques (Figure 7B). This intron part is a purine-rich element that might be essential for spliceosome binding, and leads in its absence to the exclusion of exon 3 at the transcription level.

In most of the identified Mamu-KIR1D isoforms, exon 5 was present or partially included (Figure 7A, \#1-13). Due to a 7 bp deletion in exon 5 at the gDNA level, complete inclusion of this exon at the mRNA level by constitutive splicing resulted in a frameshift that introduced a stopcodon in the beginning of exon 7 (Figure 7A; \#1, 2). The remaining transcripts that included exon 5 either skipped exon 7, but had in-frame exons 8 and 9 (Figure 7A; \#3), or had intronic/exonic inclusions subsequent to exon 6 that introduced a stopcodon (Figure 7A; \#4-6). These transcripts probably encode soluble and truncated KIR1D receptors, respectively. In other Mamu-KIR1D isoforms, the first part of exon 5 was skipped, which resulted in in-frame transcripts that encoded the D1 domain, the second part of the D2 domain, and an intact cytoplasmic tail (Figure 7A; \#7-9), or outframe transcripts that had a stopcodon subsequent to exon 5 (Figure 7A; \#10-13). Transcripts that completely lacked exon 5 encoded membrane-bound isoforms with the D1 domain and an ITIM-containing cytoplasmic tail (Figure 7A, \#14, 15), or isoforms 


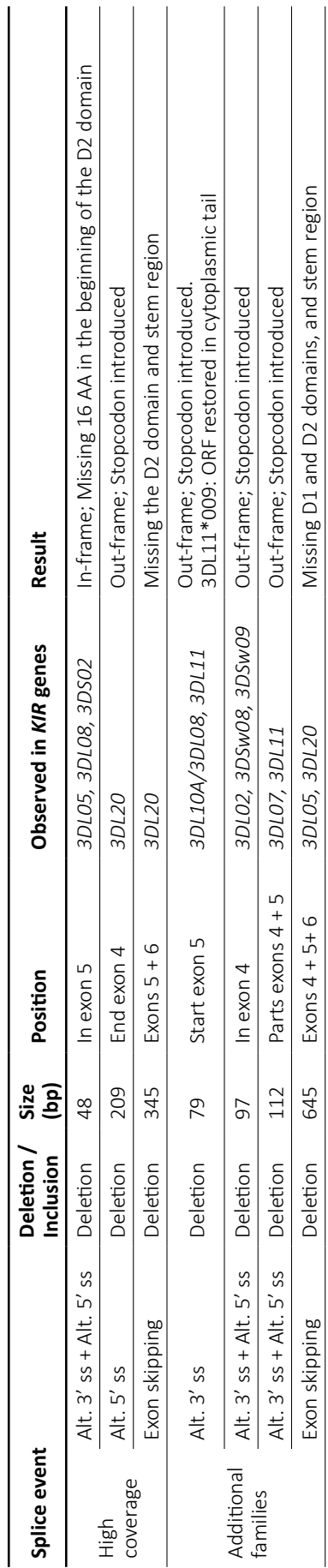

Table 3. To verify whether a complete overview of Mamu-KIR alternative splicing was obtained, four samples were pooled for a PacBio Sequel sequencing run, which provided a fourfold coverage compared to the obtained transcriptome profiles. Three additional splice events were confirmed $(\geq 10$ PacBio reads, or confirmed in two macaques), indicating that a rather complete overview of alternative splicing can be provided by data from the preceding study (9). Also, the alternative splicing profiles of three additional families, consisting of 25 rhesus macaques in total, were characterized. Only a single splice event was identified that was not observed in the main dataset, whereas 24 splice events were confirmed, indicating that most splice events are shared between rhesus macaque families.

that encoded only the D1 domain (Figure 7A, \#1618). The deletion of $36 \mathrm{bp}$ at the beginning of exon 4 , as is observed in most lineage II Mamu-KIR genes (Table 2), was also identified in multiple MamuKIR1D transcripts; these isoforms could appear with and without this splice event (Figure 7A, \#1/2, 8/9, 10/11, 12/13).

In other Old World monkey species such as cynomolgus macaques (Macaca fascicularis), olive baboons (Papio Anubis), and vervet monkeys (Chlorocebus aethiops), orthologs of Mamu-KIR1D were observed that also displayed the $5 \mathrm{bp}$ and 7 bp deletions in exons 3 and 5 , respectively $(36,71)$. In addition, a comparison of intron 2 of these genes revealed that they also lack the purine-rich element of $33 \mathrm{bp}$, which might explain the constitutive skipping of exon 3. It is not known whether these orthologs are also subjected to extensive alternative splicing. In humans, no orthologs of Mamu-KIR1D were identified. However, multiple human KIR2DS4 alleles that skip exon 3 - and that have a 22 bp deletion at the gDNA level in exon 5, which introduces a frameshift that resulted in an early stopcodon subsequent to exon 6 - have been described as Mamu-KIR1D analogs (72).

\section{Consistent alternative splicing of Mamu-KIR3DL20}

The Mamu-KIR3DL2O gene is present on most, but not all, reported Mamu-KIR haplotypes, and has been considered a framework gene. Phylogenetic analysis has illustrated a relationship 
A

\begin{tabular}{|c|c|c|c|c|c|c|c|}
\hline \multirow[t]{2}{*}{$1+2$} & 1 & 2 & 4 & 5 & 6 & 7 & \\
\hline & & & dele & \multicolumn{3}{|c|}{ Out frame } & \\
\hline \multirow[t]{2}{*}{3} & 1 & 2 & 4 & 5 & 6 & 8 & 9 \\
\hline & & & & \multicolumn{2}{|c|}{ Out frame } & & In frame \\
\hline \multirow[t]{2}{*}{4} & 1 & 2 & 4 & 5 & 6 & * & \\
\hline & & & & \multicolumn{2}{|c|}{ Out frame } & $19 \mathrm{t}$ & from intron 7 \\
\hline 5 & 1 & 2 & 4 & 5 & 6 & * & \\
\hline
\end{tabular}

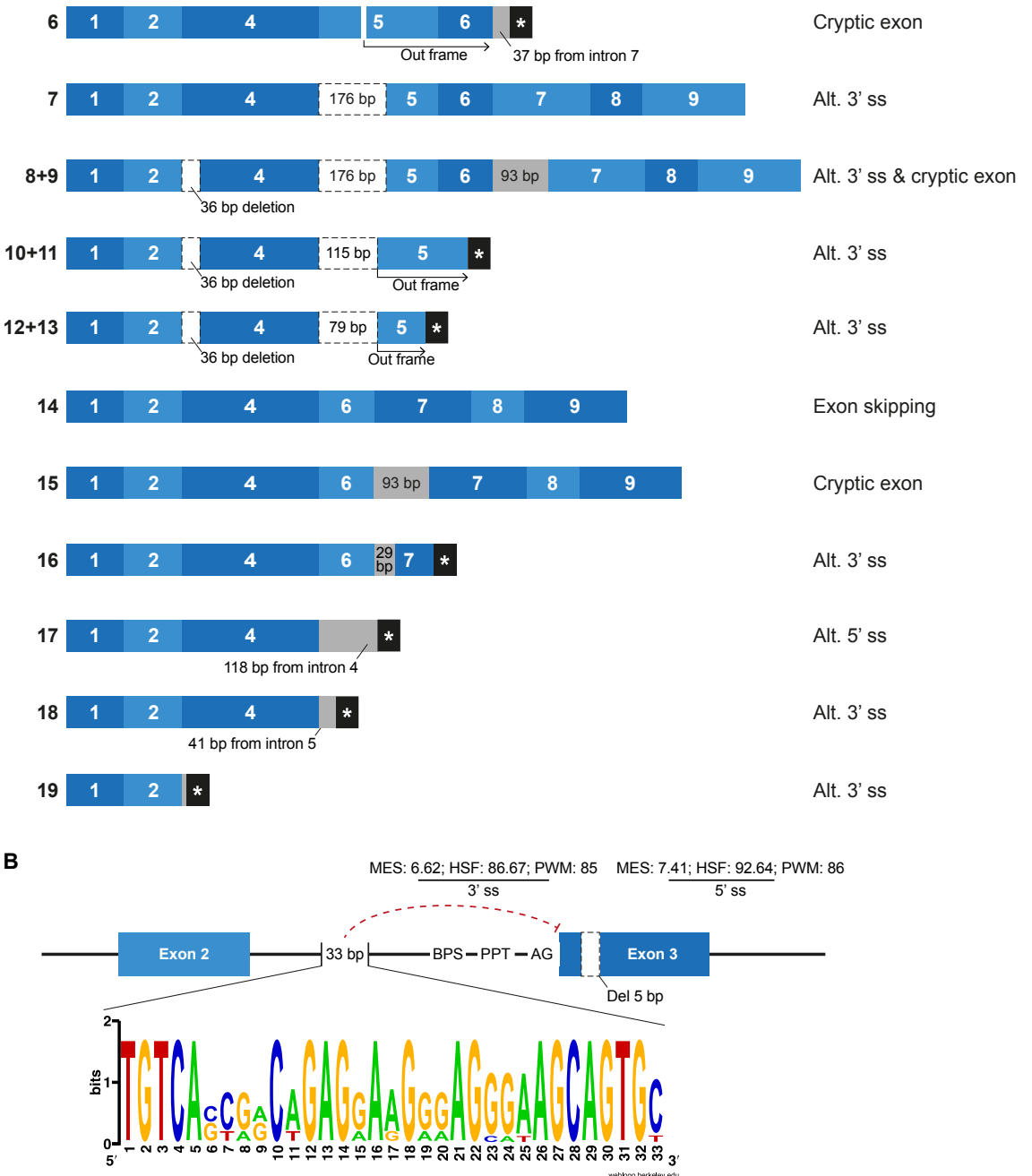

Splice mechanism

Exon skipping

Cryptic exon

Alt. 3' ss

o

Alt 3 ss

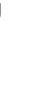


between Mamu-KIR3DL2O and human linage I (KIR2DL4, KIR2DL5) and V (KIR3DL3) KIR genes $(45,71)$. Indeed, exon 3 of Mamu-KIR3DL20 showed similarity to human KIR2DL5, and exons 4 and 5 displayed similarity to human KIR3DL3. The exons encoding the cytoplasmic tail of Mamu-KIR3DL2O are more related to macaque KIR genes. Multiple studies have suggested that the frequently identified Mamu-KIR2DLO5 transcript is a splice variant of the Mamu-KIR3DL20 gene, in which exon 4 (D1 domain) is spliced out $(33,43,44,71,73)$. Our results substantiates that Mamu-KIR2DLO5 is indeed a splice variant of MamU-KIR3DL20, and that this splice event is consistent for every identified Mamu-KIR3DL2O allele in the rhesus macaques studied (Figure 8). In addition, gel electrophoresis indicates that the amount of exon 4 skipping in MamuKIR3DL20 transcripts is considerable (additional file figure S1 in Supplementary Material). The 3' ss region of exon 4 in Mamu-KIR3DL2O is intact (MES: 8.25, HSF: 86.96, PWM: 85), although the predicted splicing strength is slightly lower compared to the 3' ss of most other macaque KIR genes (MES: 9.84, HSF: 88.52, PWM: 85). Due to a single substitution of a cytosine with a thymine, the $5^{\prime}$ ss of exon 4 in MamuKIR3DL20 alleles (MES: 6.95, HSF: 94.52, PWM: 88) also scored lower compared to the remaining KIR genes (MES: 9.22, HSF: 96.51, PWM: 92). Therefore, these suboptimal splice sites might contribute to the skipping of exon 4 in Mamu-KIR3DL20, resulting in Mamu-KIR2DLO5 transcripts. Moreover, the skipping of exon 4 might be mediated by intron 3 of Mamu-KIR3DL20, which is 450-650 bp shorter compared to intron 3 of other Mamu-KIR genes. This may result in modified or missing splicing elements in intron 3, thereby influencing the spliceosome efficiency. Of note is that in human KIR3DL3, which has an exon 4 similar to Mamu-KIR3DL20, intron 3 is also shorter, but the consistent skipping of exon 4 is not reported for this human gene. In contrast to exon 4 in all reported human and macaque KIR genes, exon 4 of Mamu-KIR3DL2O is completely conserved in all 22 reported rhesus macaque alleles. This observation suggests selective pressure, and indicates an important function of exon 4 (D1 domain)

4 Figure 7. Overview of alternative splicing in Mamu-KIR1D. (A) Nineteen different Mamu-KIR1D transcripts were observed; each transcript is illustrated by blue boxes per exon. White boxes with a dashed outline indicate partial exon deletions, and intronic inclusions are indicated in grey boxes. Multiple transcripts have an out-frame region, due to a deletion of $7 \mathrm{bp}$ in exon 5 at the gDNA level, which can introduce a stopcodon (\#1, $2,4-6)$. However, the complete inclusion of exon 5 combined with the skipping of exon 7 can restore the open reading frame (\#3). The partial deletion of exon 5 might result in Mamu-KIR1D molecules containing an intact inhibitory cytoplasmic tail (\#7-9), or in molecules that only consist of a single D1 domain (\#10-14). Transcripts that completely skipped exon 5 might encode membrane-bound molecules including an inhibitory cytoplasmic tail $(\# 14,15)$, or molecules that only encode the D1 domain, with or without the stem region (\#16-18). A second variant was observed for some transcripts, which involved the deletion of $36 \mathrm{bp}$ at the beginning of exon 4, which was also observed in lineage II KIR genes (\#1/2, 8/9, 10/11, 12/13; Table II). (B) Exon 3 is present at the gDNA level in Mamu-KIR1D, but none of the KIR1D isoforms contain the D0 domain encoded by this exon. The BPS, PPT, and both $3^{\prime}$ and $5^{\prime}$ splice sites of exon 3 are intact, just as in human lineage III KIR genes, and predicted splicing strength scores are provided. Intron 2 of all lineage III KIR genes, including MamuKIR1D, lack $33 \mathrm{bp}$ in intron 2 compared to all other KIR genes. The lack of this $33 \mathrm{bp}$ stretch might inhibit constitutive splicing of exon 3 , as indicated by the red dashed line. The weblogo plot shows the nucleotide sequence composition of this $33 \mathrm{bp}$ stretch that is present in all KIR genes except the KIR lineage III genes, which might mediate the constitutive splicing of exon 3. 


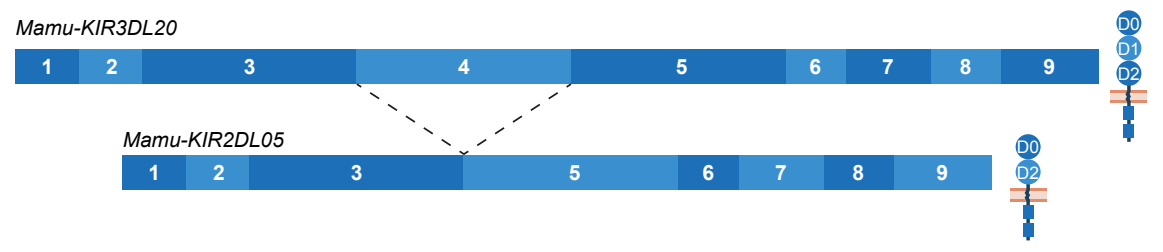

Figure 8. The skipping of exon 4 in Mamu-KIR3DL20 transcripts to generate Mamu-KIR2DLO5 transcripts. Transcripts are illustrated with the exons indicated in blue boxes, and corresponding protein structures are schematically depicted adjacent to the transcript. The splice event is indicated with dashed lines. Exon 4 (D1 domain) is consistently skipped for all studied Mamu-KIR3DL2O alleles to generate Mamu-KIR2DLO5 transcripts.

in the recognition of Mamu-KIR3DL20 receptors, or in the formation of MamuKIR2DL05 splice variants via splicing enhancer or silencer motifs.

The consensus sequence of the Mamu-KIR2DLO5 transcripts, generated by the alternative splicing of the Mamu-KIR3DL20 gene, showed $89.5 \%$ similarity to the consensus sequence of constitutively spliced human KIR2DL5 transcripts, and suggests a convergent evolution of this gene in humans and macaques. Although the exact mechanism and function of the consistent skipping of exon 4 in Mamu-KIR3DL2O resulting in Mamu-KIR2DLO5 transcripts is not completely understood, it does illustrate that alternative splicing in macaques can introduce a second KIR2DL transcript additional to Mamu-KIR2DLO4. As well as the skipping of exon 4, Mamu-KIR3DL20 transcripts that lacked exons 4 and 5 (594 bp deletion; Table 2) were also frequently observed. These transcripts were not consistently observed in all macaques, however, and seem to encode inhibitory receptors with a single extracellular domain (D0).

\section{Alternative splicing in Mamu-KIR2DL04 is mainly gene-specific}

Whereas human KIR2DL4 is a framework gene, the macaque ortholog MamuKIR2DLO4 is identified on approximately $65-75 \%$ of the reported Mamu-KIR haplotypes $(9,33)$. As with KIR2DL4 in human, the most diverse splicing profile in macaques was observed for Mamu-KIR2DLO4, including ten confirmed splice events, of which nine were gene-specific (Table 2). Exon skipping events were observed in exons 6 to 8 . In Mamu-KIR2DLO4 transcripts, the skipping of exon 7 is only observed in combination with the skipping of exon 6 (155 bp in total) (Figure 2B). The skipping of exon 8, which encodes a part of the cytoplasmic tail, was only observed in Mamu-KIR2DLO4*015. In two macaques that expressed this allele, no complete transcripts were identified, indicating allele-specific consistent exon skipping. Other events involved alternative splice sites, of which three were located in intron 7, which resulted in partial intron retentions subsequent to exon 7 (Figure 9 and Table 2). These intronic insertions introduced a stopcodon, and the three corresponding transcripts probably encode a membrane-bound receptor that lacks a cytoplasmic tail. Similar alternatively spliced transcripts were observed for human "10A" KIR2DL4 alleles with an insertion of 67 bp 


\begin{tabular}{|c|c|c|c|c|c|c|c|}
\hline 1 & 2 & 3 & 5 & 6 & 7 & 8 & 9 \\
\hline 1 & 2 & 3 & 5 & 6 & 7 & $109 \mathrm{bp}$ & \\
\hline 1 & 2 & 3 & 5 & 6 & 7 & $147 \mathrm{bp}$ & * \\
\hline
\end{tabular}
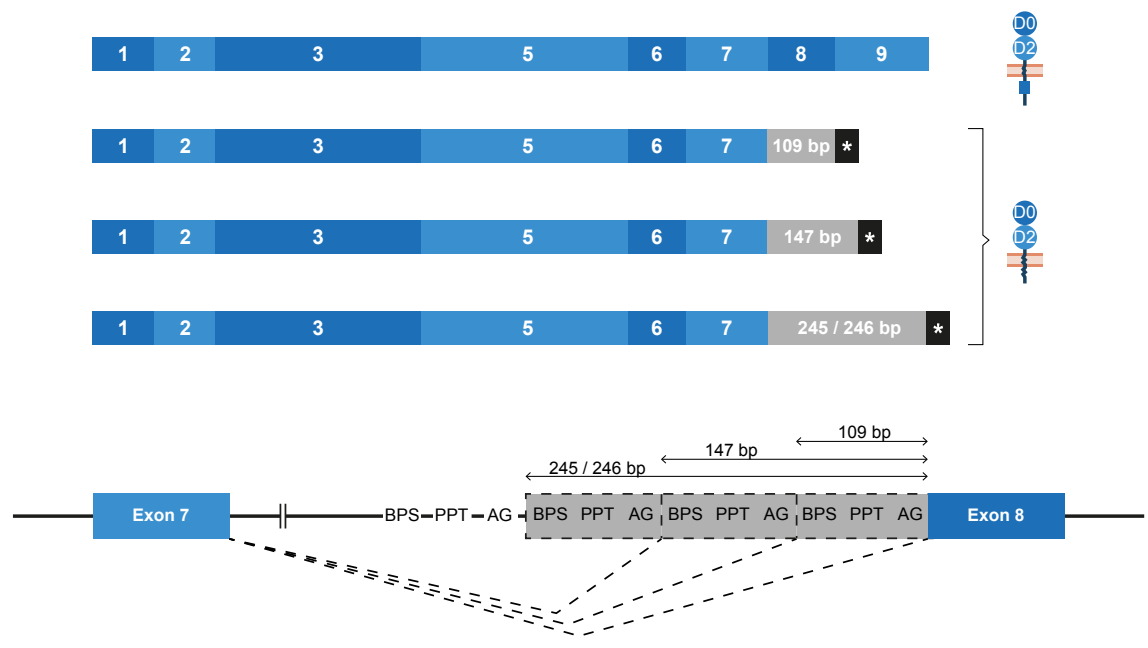

Figure 9. Alternative splice sites in intron 7 of Mamu-KIR2DL04. Transcripts are illustrated with the exons indicated in blue boxes, and corresponding protein structures are schematically depicted adjacent to the transcript. Intronic inclusions are indicated in grey boxes, and stopcodons are marked by a black box with asterisk $\left({ }^{*}\right)$. Splice events are indicated with dashed lines. Three alternative 3 ' splice sites were identified in intron 7 of Mamu-KIR2DLO4, and result in intron inclusions of 109, 147, and 245/246 bp subsequent to exon 7. All these inclusions result in a frameshift that introduces a stopcodon, indicating membrane-bound KIR2D receptors without an inhibitory cytoplasmic tail.

subsequent to exon 7 (Figure 5B). However, whereas in human KIR2DL4 the splice event is mediated by an alternative $5^{\prime}$ ss, resulting in an inclusion of the first part of intron 7 , in macaques the intron inclusions originate from the end of intron 7 , and are mediated by alternative 3 ' splice sites. Notably, three out of the four alternative splice sites in intron 7 could be identified in the human and macaque KIR2DL4 orthologs, but no similar alternatively spliced transcripts were shared between the species.

\section{Discussion}

The plasticity of the KIR genes is manifested by allelic polymorphism, copy number variation, the expansion and contraction of haplotypes, variegated expression, and the generation of hybrid genes by recombination. Here we demonstrate that alternative splicing adds an additional layer of complexity by the generation of isoforms originating from a single KIR gene. This phenomenon appears to be a structural aspect of the KIR gene cluster in different primate species. In total, 18 human and 55 macaque splice events were documented (Figures 2 and 7A, Tables 1-3), including gene-specific events, and events that were observed across different $K I R$ lineages. The potential mechanisms that mediate these splice events were categorized into common types of 
alternative splicing, and the responsible motifs were predicted and scored with different software tools. Overall, the current overview of the KIR splicing profiles in humans and rhesus macaques indicates that the generation of different KIR isoforms might be of functional relevance in health and disease.

Alternative splicing can diversify the characteristics of the encoded protein, including the domains it contains, its ligand interactions, cellular localization, and signaling properties. As a result, different isoforms encoded by the same gene can execute distinct functionalities. The receptor structure, and to a lesser extent the functional characteristics, of some KIR isoforms could be predicted based on the alternatively spliced KIR transcripts. The skipping of one or multiple exons that encode the extracellular domains (exons 3-5) likely results in the formation of KIR1D or KIR2D receptors, which can have distinct binding properties compared to the constitutively spliced isoform. A frequently observed splice event in human KIR is the skipping of exon 6 , which encodes the stem region. The function of this region is not yet clear, but it might be involved in the flexibility and orientation of the receptor. Crystallography and in vitro lysis assays illustrated, for instance, that the stem region is not involved in ligand binding, but may contribute to the inhibitory signaling function $(74,75)$. Therefore, isoforms that lack the stem region might be less stringent in delivering inhibitory signals. Transcripts that completely or partially lack exon 7 probably encode soluble receptors, whereas the absence of exons 8 and/or 9 indicates a loss of inhibitory signaling function. The consequences of events facilitated by splice mechanisms other then exon skipping is harder to predict. For example, the in-frame deletion of $36 \mathrm{bp}$ in exon 4, which is observed in thirteen different Mamu-KIR genes and mediated by an alternative $3^{\prime}$ ss, might result in a different D1 domain orientation, in distinct ligand interactions, or in an aberrant folding of the complete receptor. The conservation of this alternative $3^{\prime}$ splice site may indicate a selective pressure on a functional characteristic of the KIR isoform generated by this splice event. In vitro binding and inhibition assays with KIR isoform-transfected cells could elucidate the function of these less predictable splice variants. However, it should be noted that a large proportion of the splice events may not result in functional receptors and these product may be subjected to the nonsense-mediated decay pathway, as was previously reported in human proteomic studies (76-78). This nonproductive splicing is likely a redundancy relating to the rapid evolution of splice sites, from which beneficial isoforms are positively selected, although it has also been suggested that nonfunctional splicing is a mechanism to downregulate expression of the protein encoded by constitutive splicing $(79,80)$. Nonetheless, the number of splice events that we identified (Figures $\mathbf{2}$ and 7A, and Tables 1-3), together with the observed segregation of splice events and the sharing of splice mechanisms resulting in similar consequences on protein level in humans and rhesus macaques, suggests that at least a part of the alternative splicing profiles contributes to a structural and functional variety of KIR receptors. 
All common alternative splicing mechanisms were observed in human and rhesus macaque KIR genes, except for intron retention, which was only observed in a single splice event in Mamu-KIR2DLO4. In both species, similar exon skipping events were observed (Figure 2 and Tables 1-3), although in humans more events involved the skipping of multiple exons, especially exon 7 and its flanking exons. In addition to most exon skipping events, only the deletion of $198 \mathrm{bp}$ in the end of exon 3, which is mediated by an alternative 5' ss, was shared between humans and rhesus macaques. In macaques, this deletion was observed in three different genes, whereas in humans this splice event was specific for KIR2DL4, and was only observed in combination with a second deletion in the transmembrane region. These isoforms probably have an aberrant D0 domain, which might result in modified binding properties. All other splice events were only identified in one of the two species. In contrast to human KIR splice events, most splice events in macaque KIR involved the domain-encoding exons. This could be related to the expansion of lineage II KIR genes in macaques, which contain three extracellular domains, and therefore might have more flexibility to modify the domains without compromising ligand binding.

The skipping of exon 4 (D1 domain) was consistently observed in transcripts of Mamu-KIR3DL2O (lineage V), which is considered a framework gene in rhesus macaques, and resulted in Mamu-KIR2D transcripts that only encoded the D0 and D2 domains. These alternatively spliced transcripts seem to be a functional analog of human KIR2DL5 (lineage I) and they share a similarity of $89.5 \%$, suggesting a convergent evolution of this structure. In macaques, the Mamu-KIR2DL05 transcripts were identified in all individuals, whereas in humans, KIR2DL5 is only present on specific haplotypes (group B haplotypes). This is an indication that the alternatively spliced Mamu-KIR2DLO5 transcripts, or the Mamu-KIR3DL2O gene itself, are essential to rhesus macaques. The complete conservation of exon 4 in all Mamu-KIR3DL2O alleles further supports this. Exon 4 in Mamu-KIR3DL2O seems to be essential in facilitating its own consistent skipping, or in the interaction of Mamu-KIR3DL20 molecules, and might therefore be conserved by selective pressure. This conserved character is not observed for any other exon in human or macaque KIR. The skipping of exon 4 in Mamu-KIR3DL20 transcripts illustrates how alternative splicing can expand the plasticity of the KIR repertoire by generating isoforms of two different $K I R$ lineages from a single gene.

The skipping of exon 5, and exons 4 and 5 together, was observed in KIR genes of humans and macaques, and the events might have similar consequences. The skipping of exon 3 was not observed in human and macaque lineage I and II KIR genes, which implies essential properties for the DO domain. It has been reported that the DO domain is involved in the direct binding of MHC class I molecules (81), whereas others described only a modulatory role for this domain in $\operatorname{KIR} 3 D$ receptors $(82,83)$. Furthermore, the cell surface level of human KIR3DL1 could be modified by D0 polymorphisms, such as the substitution of a valine with a leucine at position 18 (V18L), which prevents the surface expression of KIR3DL1*053 (84). The characteristics of the D0 domain might be essential for KIR3D function in both species, and therefore the alternative splicing of this domain might be subjected to negative selection. 
A large number of alternative splicing events were observed for the Mamu-KIR1D gene (Figure 7A), which is the only lineage III KIR gene in macaques and is highly conserved, as only three alleles have been documented in the apparent functional sections of the gene (exons 1, 2 and 4). Despite an intact BPS, PPT, and 3' and 5' splice sites, exon 3 is constitutively skipped in this gene. An explanation for this phenomenon can be found in the absence of a purine-rich stretch of $33 \mathrm{bp}$ in intron 2 of MamuKIR1D (Figure 7B), which is also lacking in human lineage III KIR genes. Human and macaque KIR genes, which do include exon 3 in their transcripts, have an intron 2 that contains the purine-rich $33 \mathrm{bp}$. Within these genes, the $33 \mathrm{bp}$ appear highly conserved, which might indicate its essential role for spliceosome recognition. Furthermore, the Mamu-KIR1D gene shows extensive alternative splicing subsequent to exon 4, and this might be due to the introns flanking exons 5 and 6 . In rhesus macaques, introns 5 and 6 of lineage II KIR genes are approximately 2000 bp and 914 bp in length, respectively. Introns 5 and 6 of Mamu-KIR1D (lineage III) are approximately $3290 \mathrm{bp}$ and $4330 \mathrm{bp}$, respectively, and similar intron lengths are observed in human lineage III KIR genes. In humans, lineage III KIR genes mainly interact with HLA-C molecules. In rhesus macaques, however, no homolog of HLA-C is identified, and most Mamu-KIR probably interacts with members of the expanded repertoire of MHC-A and -B molecules. Therefore, selective pressure to conserve the lineage III gene might be low, which makes the large introns observed for Mamu-KIR1D prone to mutations that might induce alternative splicing. As such, an initial macaque KIR2D gene is now translated into Mamu-KIR1D. At present it is unclear whether any of the different identified Mamu-KIR1D isoforms are functional in macaques.

The extent of the impact that alternative splicing has on the KIR repertoire is dependent not only on which splice variants are formed but also on the frequency of the splice events. In this study, the PacBio Sequel platform was used to determine the alternative splicing profiles, but this method does not provide quantification, and may only be used as a quantitative indication. Approximately $4 \%$ and $53 \%$ of the 100\%-matched human KIR2D/3D and KIR2DL4 reads accounted for alternatively spliced transcripts. In rhesus macaques, $24 \%$ and $13 \%$ splice variants were obtained from the 100\%-matched KIR2D/3D and KIR2DL04 PacBio reads, respectively. These percentages indicate abundant alternative splicing for human KIR2DL4 and macaque $K I R 2 D / 3 D$, but one has to be cautious with the interpretation of these numbers, as the quantification value of the PacBio platform is low. Furthermore, preferential amplification of the used primer sets can not be ruled out, which may also have an effect on the calculated numbers. More reliable quantification methods, like droplet digital PCR (ddPCR) or RNA-seq, are hard to adapt on a multigene family such as KIR. Therefore, we are currently only able to provide quantitative indications of alternative KIR splicing.

In this study, and in other studies that reported KIR isoforms (28-30, 36, 42, 43), the splice variants were identified in whole blood samples, and might therefore give a representation of the complete splicing profile. However, tissue-specific alternative splicing has been reported, and suggests isoforms with a local specialized function 
(85). Especially in the brain, testis, and liver, increased alternative splicing events can be identified, which mainly involved exon skipping and alternative splice sites. NK cells that reside in tissues are reported in multiple organs, such as the intestines, lungs, liver, spleen, lymph nodes, brain, eye retina, and uterus, and can be phenotypically and functionally distinct from the NK cells in peripheral blood (86-93). The diversity of NKcell subsets in the different tissues includes selective expression of KIR, but might also involve distinct alternative splicing profiles of the KIR genes. The regulation of tissuespecific alternative splicing is complex, and involves the differential expression of splicing factors and epigenetic modifications, such as methylation and histone acetylation (94-96). In tissues that should dampen the immune response to avoid inflammation, like the eye retina, or tissues that require high immune surveillance, like the intestines and liver, alternative splicing might provide the required isoforms. Also in the uterine tissue, phenotypically and functionally distinct NK cells (uNK cells) have been identified that mainly express KIR2DL4, and are involved in pregnancy. This NK cell subset can interact with the highly expressed HLA-G molecules in the uterus, which are subjected to alternative splicing, to maintain the fetal-maternal interface and induce cytokine production (97-99). Alternative splicing might modify the activity and interactions of KIR2DL4 expressed on the UNK cells, for example, by skipping the transmembrane region to generate soluble KIR2DL4 receptors (Figure 4), or by an insertion of $67 \mathrm{bp}$ that can regulate the presence of an inhibitory cytoplasmic tail (Figure 5B).

Overall, we characterized the alternative splicing profiles of $K I R$ genes in human and macaque families, which provides an illustration of the potential formation of protein isoforms. These posttranscriptional modifications might contribute to the complexity of the KIR gene family of both species, human and macaque, and result in a wide structural and functional variety of receptors that might be involved in health and disease.

\section{Acknowledgements}

We thank D. Devine for editing the manuscript and F. van Hassel for preparing the figures. Human PBMC samples from a preceding study (9) were kindly provided by Prof. dr. Frans H. J. Claas (Immunohematology and Blood Transfusion, Leiden University Medical Center, Leiden, The Netherlands). 


\section{References}

1. Giebel, S., Locatelli, F., Lamparelli, T., Velardi, A., Davies, S., Frumento, G., et al. (2003). Survival advantage with KIR ligand incompatibility in hematopoietic stem cell transplantation from unrelated donors. Blood 102(3), 814819. doi: 10.1182/blood-2003-01-0091.

2. Hiby, S.E., Walker, J.J., O’Shaughnessy K, M., Redman, C.W., Carrington, M., Trowsdale, J., et al. (2004). Combinations of maternal KIR and fetal HLA-C genes influence the risk of preeclampsia and reproductive success. J Exp Med 200(8), 957-965. doi: 10.1084/jem.20041214.

3. Kulkarni, S., Martin, M.P., and Carrington, M. (2008). The Ying and Yang of HLA and KIR in Human Disease. Semin Immunol 20(6), 343-352. doi: 10.1016/j.smim.2008.06.003.

4. Rajagopalan, S., and Long, E.O. (2005). Understanding how combinations of HLA and KIR genes influence disease. J Exp Med 201(7), 1025-1029. doi: 10.1084/jem.20050499.

5. Robinson, J., Mistry, K., McWilliam, H., Lopez, R., and Marsh, S.G.E. (2010). IPD-the Immuno Polymorphism Database. Nucleic Acids Res 38(Database issue), D863-869. doi: 10.1093/nar/gkp879.

6. Uhrberg, M., Valiante, N.M., Shum, B.P., Shilling, H.G., Lienert-Weidenbach, K., Corliss, B., et al. (1997). Human diversity in killer cell inhibitory receptor genes. Immunity 7(6), 753-763.

7. Held, W., and Kunz, B. (1998). An allele-specific, stochastic gene expression process controls the expression of multiple Ly49 family genes and generates a diverse, MHC-specific NK cell receptor repertoire. Eur J Immunol 28(8), 2407-2416. doi: 10.1002/(sici)1521-4141(199808)28:08\&\#60;2407::aid-immu2407\&\#62;3.0.co;2-d.

8. Valiante, N.M., Uhrberg, M., Shilling, H.G., Lienert-Weidenbach, K., Arnett, K.L., D’Andrea, A., et al. (1997). Functionally and structurally distinct NK cell receptor repertoires in the peripheral blood of two human donors. Immunity 7(6), 739-751.

9. Bruijnesteijn, J., van der Wiel, M.K.H., Swelsen, W.T.N., Otting, N., de Vos-Rouweler, A.J.M., Elferink, D., et al. (2018). Human and Rhesus Macaque KIR Haplotypes Defined by Their Transcriptomes. J Immunol. doi: 10.4049/jimmunol.1701480.

10. Jiang, W., Johnson, C., Jayaraman, J., Simecek, N., Noble, J., Moffatt, M.F., et al. (2012). Copy number variation leads to considerable diversity for B but not A haplotypes of the human KIR genes encoding NK cell receptors. Genome Res 22(10), 1845-1854. doi: 10.1101/gr.137976.112.

11. Traherne, J.A., Martin, M., Ward, R., Ohashi, M., Pellett, F., Gladman, D., et al. (2010). Mechanisms of copy number variation and hybrid gene formation in the KIR immune gene complex. Hum Mol Genet 19(5), 737-751. doi: $10.1093 / \mathrm{hmg} / \mathrm{ddp} 538$.

12. Martin, A.M., Freitas, E.M., Witt, C.S., and Christiansen, F.T. (2000). The genomic organization and evolution of the natural killer immunoglobulin-like receptor (KIR) gene cluster. Immunogenetics 51(4-5), 268-280.

13. Vilches, C., and Parham, P. (2002). KIR: diverse, rapidly evolving receptors of innate and adaptive immunity. Annu Rev Immunol 20, 217-251. doi: 10.1146/annurev.immunol.20.092501.134942.

14. Mingari, M.C., Moretta, A., and Moretta, L. (1998). Regulation of KIR expression in human T cells: a safety mechanism that may impair protective T-cell responses. Immunol Today 19(4), 153-157.

15. Roe, D., Vierra-Green, C., Pyo, C.W., Eng, K., Hall, R., Kuang, R., et al. (2017). Revealing complete complex KIR haplotypes phased by long-read sequencing technology. Genes Immun 18(3), 127-134. doi: 10.1038/ gene.2017.10.

16. Robinson, J., Halliwell, J.A., McWilliam, H., Lopez, R., and Marsh, S.G. (2013). IPD--the Immuno Polymorphism Database. Nucleic Acids Res 41(Database issue), D1234-1240. doi: 10.1093/nar/gks1140.

17. Pyo, C.W., Wang, R., Vu, Q., Cereb, N., Yang, S.Y., Duh, F.M., et al. (2013). Recombinant structures expand and contract inter and intragenic diversification at the KIR locus. BMC Genomics 14, 89. doi: 10.1186/1471-216414-89.

18. Nilsen, T.W., and Graveley, B.R. (2010). Expansion of the eukaryotic proteome by alternative splicing. Nature 463(7280), 457-463. doi: 10.1038/nature08909.

19. Blencowe, B.J. (2006). Alternative splicing: new insights from global analyses. Cell 126(1), 37-47. doi: 10.1016/j. cell.2006.06.023.

20. Will, C.L., and Luhrmann, R. (2011). Spliceosome structure and function. Cold Spring Harb Perspect Bio/ 3(7). doi: 10.1101/cshperspect.a003707. 
21. Pagani, F., and Baralle, F.E. (2004). Genomic variants in exons and introns: identifying the splicing spoilers. Nat Rev Genet 5(5), 389-396. doi: 10.1038/nrg1327.

22. Roy, B., Haupt, L.M., and Griffiths, L.R. (2013). Review: Alternative Splicing (AS) of Genes As An Approach for Generating Protein Complexity. Curr Genomics 14(3), 182-194. doi: 10.2174/1389202911314030004.

23. Tazi, J., Bakkour, N., and Stamm, S. (2009). Alternative splicing and disease. Biochim Biophys Acta 1792(1), $14-$ 26. doi: $10.1016 / \mathrm{j}$.bbadis.2008.09.017.

24. Venables, J.P. (2006). Unbalanced alternative splicing and its significance in cancer. Bioessays 28(4), 378-386 doi: 10.1002/bies.20390

25. Skotheim, R.I., and Nees, M. (2007). Alternative splicing in cancer: noise, functional, or systematic? Int J Biochem Cell Biol 39(7-8), 1432-1449. doi: 10.1016/j.biocel.2007.02.016.

26. Voorter, C.E., Gerritsen, K.E., Groeneweg, M., Wieten, L., and Tilanus, M.G. (2016). The role of gene polymorphism in HLA class I splicing. Int J Immunogenet 43(2), 65-78. doi: 10.1111/iji.12256.

27. Romero, A., García-García, F., López-Perolio, I., Ruiz de Garibay, G., García-Sáenz, J.A., Garre, P., et al. (2015). BRCA1 Alternative splicing landscape in breast tissue samples. BMC Cancer 15(1), 219. doi: 10.1186/s12885015-1145-9.

28. Chwae, Y.J., Cho, S.E., Kim, S.J., and Kim, J. (1999). Diversity of the repertoire of p58 killer cell inhibitory receptors in a single individual. Immunol Lett 68(2-3), 267-274

29. Dohring, C., Samaridis, J., and Colonna, M. (1996). Alternatively spliced forms of human killer inhibitory receptors. Immunogenetics 44(3), 227-230

30. Goodridge, J.P., Lathbury, L.J., Steiner, N.K., Shulse, C.N., Pullikotil, P., Seidah, N.G., et al. (2007). Three common alleles of KIR2DL4 (CD158d) encode constitutively expressed, inducible and secreted receptors in NK cells. Eur J Immunol 37(1), 199-211. doi: 10.1002/eji.200636316.

31. Vilches, C., Rajalingam, R., Uhrberg, M., Gardiner, C.M., Young, N.T., and Parham, P. (2000). KIR2DL5, a novel killer-cell receptor with a D0-D2 configuration of Ig-like domains. J Immunol 164(11), 5797-5804.

32. Goodridge, J.P., Witt, C.S., Christiansen, F.T., and Warren, H.S. (2003). KIR2DL4 (CD158d) genotype influences expression and function in NK cells. J Immunol 171(4), 1768-1774.

33. Blokhuis, J.H., van der Wiel, M.K., Doxiadis, G.G., and Bontrop, R.E. (2010). The mosaic of KIR haplotypes in rhesus macaques. Immunogenetics 62(5), 295-306. doi: 10.1007/s00251-010-0434-3.

34. Guethlein, L.A., Norman, P.J., Heijmans, C.M., de Groot, N.G., Hilton, H.G., Babrzadeh, F., et al. (2017). Two Orangutan Species Have Evolved Different KIR Alleles and Haplotypes. J Immunol 198(8), 3157-3169. doi: 10.4049/jimmunol.1602163.

35. Guethlein, L.A., Older Aguilar, A.M., Abi-Rached, L., and Parham, P. (2007). Evolution of killer cell Ig-like receptor (KIR) genes: definition of an orangutan KIR haplotype reveals expansion of lineage III KIR associated with the emergence of MHC-C. J Immunol 179(1), 491-504

36. Prall, T.M., Graham, M.E., Karl, J.A., Wiseman, R.W., Ericsen, A.J., Raveendran, M., et al. (2017). Improved full-length killer cell immunoglobulin-like receptor transcript discovery in Mauritian cynomolgus macaques. Immunogenetics 69(5), 325-339. doi: 10.1007/s00251-017-0977-7.

37. Rajalingam, R., Hong, M., Adams, E.J., Shum, B.P., Guethlein, L.A., and Parham, P. (2001). Short KIR haplotypes in pygmy chimpanzee (Bonobo) resemble the conserved framework of diverse human KIR haplotypes. J Exp Med 193(1), 135-146.

38. Bontrop, R.E. (2001). Non-human primates: essential partners in biomedical research. Immunol Rev 183, 5-9.

39. Kaushal, D., Mehra, S., Didier, P.J., and Lackner, A.A. (2012). The non-human primate model of tuberculosis. J Med Primatol 41(3), 191-201. doi: 10.1111/j.1600-0684.2012.00536.x.

40. Vierboom, M.P.M., Jonker, M., Bontrop, R.E., and t Hart, B. (2005). Modeling human arthritic diseases in nonhuman primates. Arthritis Res Ther 7(4), 145-154. doi: 10.1186/ar1773.

41. de Groot, N.G., Blokhuis, J.H., Otting, N., Doxiadis, G.G., and Bontrop, R.E. (2015). Co-evolution of the MHC class I and KIR gene families in rhesus macaques: ancestry and plasticity. Immunol Rev 267(1), 228-245. doi: 10.1111/imr.12313.

42. Moreland, A.J., Guethlein, L.A., Reeves, R.K., Broman, K.W., Johnson, R.P., Parham, P., et al. (2011). Characterization of killer immunoglobulin-like receptor genetics and comprehensive genotyping by pyrosequencing in rhesus macaques. BMC Genomics 12, 295. doi: 10.1186/1471-2164-12-295. 
43. Hershberger, K.L., Shyam, R., Miura, A., and Letvin, N.L. (2001). Diversity of the killer cell Ig-like receptors of rhesus monkeys. J Immunol 166(7), 4380-4390.

44. Bimber, B.N., Moreland, A.J., Wiseman, R.W., Hughes, A.L., and O'Connor, D.H. (2008). Complete Characterization of Killer Ig-Like Receptor (KIR) Haplotypes in Mauritian Cynomolgus Macaques: Novel Insights into Nonhuman Primate KIR Gene Content and Organization. J Immunol 181(9), 6301-6308.

45. Sambrook, J.G., Bashirova, A., Palmer, S., Sims, S., Trowsdale, J., Abi-Rached, L., et al. (2005). Single haplotype analysis demonstrates rapid evolution of the killer immunoglobulin-like receptor (KIR) loci in primates. Genome Res 15(1), 25-35. doi: 10.1101/gr.2381205.

46. Kearse, M., Moir, R., Wilson, A., Stones-Havas, S., Cheung, M., Sturrock, S., et al. (2012). Geneious Basic: an integrated and extendable desktop software platform for the organization and analysis of sequence data. Bioinformatics 28(12), 1647-1649. doi: 10.1093/bioinformatics/bts199.

47. Blokhuis, J.H., Doxiadis, G.G., and Bontrop, R.E. (2009). A splice site mutation converts an inhibitory killer cell Ig-like receptor into an activating one. Mol Immunol 46(4), 640-648. doi: 10.1016/j.molimm.2008.08.270.

48. Colantonio, A.D., Bimber, B.N., Neidermyer, W.J., Jr., Reeves, R.K., Alter, G., Altfeld, M., et al. (2011). KIR polymorphisms modulate peptide-dependent binding to an MHC class I ligand with a Bw6 motif. PLoS Pathog 7(3), e1001316. doi: 10.1371/journal.ppat.1001316.

49. Bostik, P., Kobkitjaroen, J., Tang, W., Villinger, F., Pereira, L.E., Little, D.M., et al. (2009). Decreased NK cell frequency and function is associated with increased risk of KIR3DL allele polymorphism in simian immunodeficiency virus-infected rhesus macaques with high viral loads. J Immunol 182(6), 3638-3649. doi: 10.4049/jimmunol.0803580.

50. Grendell, R.L., Hughes, A.L., and Golos, T.G. (2001). Cloning of rhesus monkey killer-cell Ig-like receptors (KIRs) from early pregnancy decidua. Tissue Antigens 58(5), 329-334.

51. Kruse, P.H., Rosner, C., and Walter, L. (2010). Characterization of rhesus macaque KIR genotypes and haplotypes. Immunogenetics 62(5), 281-293. doi: 10.1007/s00251-010-0433-4.

52. Robinson, J., Waller, M.J., Stoehr, P., and Marsh, S.G. (2005). IPD--the Immuno Polymorphism Database. Nucleic Acids Res 33(Database issue), D523-526. doi: 10.1093/nar/gki032.

53. Jian, X., Boerwinkle, E., and Liu, X. (2014). In silico tools for splicing defect prediction: a survey from the viewpoint of end users. Genet Med 16(7), 497-503. doi: 10.1038/gim.2013.176.

54. Ohno, K., Takeda, J.I., and Masuda, A. (2018). Rules and tools to predict the splicing effects of exonic and intronic mutations. Wiley Interdiscip Rev RNA 9(1). doi: 10.1002/wrna.1451.

55. Houdayer, C., Caux-Moncoutier, V., Krieger, S., Barrois, M., Bonnet, F., Bourdon, V., et al. (2012). Guidelines for splicing analysis in molecular diagnosis derived from a set of 327 combined in silico/in vitro studies on BRCA1 and BRCA2 variants. Hum Mutat 33(8), 1228-1238. doi: 10.1002/humu.22101.

56. Jian, X., Boerwinkle, E., and Liu, X. (2014). In silico prediction of splice-altering single nucleotide variants in the human genome. Nucleic Acids Res 42(22), 13534-13544. doi: 10.1093/nar/gku1206.

57. Colombo, M., De Vecchi, G., Caleca, L., Foglia, C., Ripamonti, C.B., Ficarazzi, F., et al. (2013). Comparative in vitro and in silico analyses of variants in splicing regions of BRCA1 and BRCA2 genes and characterization of novel pathogenic mutations. PLoS One 8(2), e57173. doi: 10.1371/journal.pone.0057173.

58. Yeo, G., and Burge, C.B. (2004). Maximum Entropy Modeling of Short Sequence Motifs with Applications to RNA Splicing Signals. Journal of Computational Biology 11(2-3), 377-394. doi: 10.1089/1066527041410418.

59. Rogozin, I.B., and Milanesi, L. (1997). Analysis of donor splice sites in different eukaryotic organisms. J Mol Evol 45(1), 50-59.

60. Desmet, F.O., Hamroun, D., Lalande, M., Collod-Beroud, G., Claustres, M., and Beroud, C. (2009). Human Splicing Finder: an online bioinformatics tool to predict splicing signals. Nucleic Acids Res 37(9), e67. doi: 10.1093/nar/gkp215.

61. Reese, M.G., Eeckman, F.H., Kulp, D., and Haussler, D. (1997). Improved splice site detection in Genie. J Comput Biol 4(3), 311-323. doi: 10.1089/cmb.1997.4.311.

62. Keren, H., Lev-Maor, G., and Ast, G. (2010). Alternative splicing and evolution: diversification, exon definition and function. Nat Rev Genet 11(5), 345-355. doi: 10.1038/nrg2776. 
63. Schwartz, S.H., Silva, J., Burstein, D., Pupko, T., Eyras, E., and Ast, G. (2008). Large-scale comparative analysis of splicing signals and their corresponding splicing factors in eukaryotes. Genome Res 18(1), 88-103. doi: 10.1101/ gr.6818908.

64. Cornish-Bowden, A. (1985). Nomenclature for incompletely specified bases in nucleic acid sequences: recommendations 1984. Nucleic Acids Res 13(9), 3021-3030.

65. Coolidge, C.J., Seely, R.J., and Patton, J.G. (1997). Functional analysis of the polypyrimidine tract in pre-mRNA splicing. Nucleic Acids Res 25(4), 888-896.

66. Sickmier, E.A., Frato, K.E., Shen, H., Paranawithana, S.R., Green, M.R., and Kielkopf, C.L. (2006). Structural basis for polypyrimidine tract recognition by the essential pre-mRNA splicing factor U2AF65. Mol Cell 23(1), 49-59 doi: 10.1016/j.molcel.2006.05.025.

67. Dhir, A., and Buratti, E. (2010). Alternative splicing: role of pseudoexons in human disease and potential therapeutic strategies. Febs j 277(4), 841-855. doi: 10.1111/j.1742-4658.2009.07520.x.

68. Rump, A., Rosen-Wolff, A., Gahr, M., Seidenberg, J., Roos, C., Walter, L., et al. (2006). A splice-supporting intronic mutation in the last bp position of a cryptic exon within intron 6 of the CYBB gene induces its incorporation into the mRNA causing chronic granulomatous disease (CGD). Gene 371(2), 174-181. doi: 10.1016/j.gene.2005.11.036.

69. Fackenthal, J.D., Lee, Y., and Olopade, O.I. (2012). Hidden dangers: a cryptic exon disrupts BRCA2 mRNA. Clin Cancer Res 18(18), 4865-4867. doi: 10.1158/1078-0432.ccr-12-2090.

70. Wright, P.W., Li, H., Huehn, A., O'Connor, G.M., Cooley, S., Miller, J.S., et al. (2014). Characterization of a weakly expressed KIR2DL1 variant reveals a novel upstream promoter that controls KIR expression. Genes Immun 15(7), 440-448. doi: 10.1038/gene.2014.34.

71. Palacios, C., Cuervo, L.C., and Cadavid, L.F. (2011). Evolutionary patterns of killer cell Ig-like receptor genes in Old World monkeys. Gene 474(1-2), 39-51. doi: 10.1016/j.gene.2010.12.006.

72. Maxwell, L.D., Wallace, A., Middleton, D., and Curran, M.D. (2002). A common KIR2DS4 deletion variant in the human that predicts a soluble KIR molecule analogous to the KIR1D molecule observed in the rhesus monkey. Tissue Antigens 60(3), 254-258.

73. Bimber, B.N., and Evans, D.T. (2015). The killer-cell immunoglobulin-like receptors of macaques. Immunol Rev 267(1), 246-258. doi: 10.1111/imr.12329.

74. Fan, Q.R., Long, E.O., and Wiley, D.C. (2000). A disulfide-linked natural killer cell receptor dimer has higher affinity for HLA-C than wild-type monomer. Eur J Immuno/ 30(9), 2692-2697. doi: 10.1002/1521-4141(200009)30:9<2692::aid-immu2692>3.0.co;2-0.

75. Kumar, S., Rajagopalan, S., Sarkar, P., Dorward, D.W., Peterson, M.E., Liao, H.S., et al. (2016). Zinc-Induced Polymerization of Killer-Cell Ig-like Receptor into Filaments Promotes Its Inhibitory Function at Cytotoxic Immunological Synapses. Mol Cell 62(1), 21-33. doi: 10.1016/j.molcel.2016.03.009.

76. Pickrell, J.K., Pai, A.A., Gilad, Y., and Pritchard, J.K. (2010). Noisy splicing drives mRNA isoform diversity in human cells. PLoS Genet 6(12), e1001236. doi: 10.1371/journal.pgen.1001236.

77. Leoni, G., Le Pera, L., Ferre, F., Raimondo, D., and Tramontano, A. (2011). Coding potential of the products of alternative splicing in human. Genome Biol 12(1), R9. doi: 10.1186/gb-2011-12-1-r9.

78. Sorek, R., Shamir, R., and Ast, G. (2004). How prevalent is functional alternative splicing in the human genome? Trends Genet 20(2), 68-71. doi: 10.1016/j.tig.2003.12.004.

79. Lewis, B.P., Green, R.E., and Brenner, S.E. (2003). Evidence for the widespread coupling of alternative splicing and nonsense-mediated mRNA decay in humans. Proc Natl Acad Sci U S A 100(1), 189-192. doi: 10.1073/ pnas. 0136770100

80. Lareau, L.F., Brooks, A.N., Soergel, D.A., Meng, Q., and Brenner, S.E. (2007). The coupling of alternative splicing and nonsense-mediated mRNA decay. Adv Exp Med Biol 623, 190-211.

81. Sharma, D., Bastard, K., Guethlein, L.A., Norman, P.J., Yawata, N., Yawata, M., et al. (2009). Dimorphic motifs in $\mathrm{D} 0$ and D1+D2 domains of killer cell Ig-like receptor 3DL1 combine to form receptors with high, moderate, and no avidity for the complex of a peptide derived from HIV and HLA-A*2402. J Immunol 183(7), 4569-4582. doi: 10.4049/jimmunol.0901734.

82. Khakoo, S.I., Geller, R., Shin, S., Jenkins, J.A., and Parham, P. (2002). The D0 domain of KIR3D acts as a major histocompatibility complex class I binding enhancer. J Exp Med 196(7), 911-921. 
83. O'Connor, G.M., Vivian, J.P., Widjaja, J.M., Bridgeman, J.S., Gostick, E., Lafont, B.A., et al. (2014). Mutational and structural analysis of KIR3DL1 reveals a lineage-defining allotypic dimorphism that impacts both HLA and peptide sensitivity. J Immunol 192(6), 2875-2884. doi: 10.4049/jimmunol.1303142.

84. Thomas, R., Yamada, E., Alter, G., Martin, M.P., Bashirova, A.A., Norman, P.J., et al. (2008). Novel KIR3DL1 alleles and their expression levels on NK cells: convergent evolution of KIR3DL1 phenotype variation? J Immunol 180(10), 6743-6750.

85. Yeo, G., Holste, D., Kreiman, G., and Burge, C.B. (2004). Variation in alternative splicing across human tissues. Genome Biol 5(10), R74. doi: 10.1186/gb-2004-5-10-r74.

86. Peng, H., Wisse, E., and Tian, Z. (2016). Liver natural killer cells: subsets and roles in liver immunity. Cell Mol Immunol 13(3), 328-336. doi: 10.1038/cmi.2015.96.

87. Burt, B.M., Plitas, G., Zhao, Z., Bamboat, Z.M., Nguyen, H.M., Dupont, B., et al. (2009). The Iytic potential of human liver NK cells is restricted by their limited expression of inhibitory killer Ig-like receptors. J Immunol 183(3), 1789-1796. doi: 10.4049/jimmunol.0900541.

88. Hesker, P.R., and Krupnick, A.S. (2013). The role of natural killer cells in pulmonary immunosurveillance. Front Biosci (Schol Ed) 5, 575-587.

89. Sanos, S.L., and Diefenbach, A. (2010). Isolation of NK cells and NK-like cells from the intestinal lamina propria. Methods Mol Biol 612, 505-517. doi: 10.1007/978-1-60761-362-6_32.

90. Garrod, K.R., Wei, S.H., Parker, I., and Cahalan, M.D. (2007). Natural killer cells actively patrol peripheral lymph nodes forming stable conjugates to eliminate MHC-mismatched targets. Proc Natl Acad Sci U S A 104(29), 12081-12086. doi: 10.1073/pnas.0702867104.

91. Niederkorn, J.Y. (2010). “NK cells in the eye," in Natural Killer Cells. Elsevier Ltd.), pp. 385-401

92. Gaynor, L.M., and Colucci, F. (2017). Uterine Natural Killer Cells: Functional Distinctions and Influence on Pregnancy in Humans and Mice. Front Immunol 8, 467. doi: 10.3389/fimmu.2017.00467.

93. Carrega, P., and Ferlazzo, G. (2012). Natural killer cell distribution and trafficking in human tissues. Front Immuno/ 3, 347. doi: 10.3389/fimmu.2012.00347.

94. Chen, M., and Manley, J.L. (2009). Mechanisms of alternative splicing regulation: insights from molecular and genomics approaches. Nat Rev Mol Cell Biol 10(11), 741-754. doi: 10.1038/nrm2777.

95. Grosso, A.R., Gomes, A.Q., Barbosa-Morais, N.L., Caldeira, S., Thorne, N.P., Grech, G., et al. (2008). Tissuespecific splicing factor gene expression signatures. Nucleic Acids Res 36(15), 4823-4832. doi: 10.1093/nar/ gkn463.

96. Zhou, H.L., Luo, G., Wise, J.A., and Lou, H. (2014). Regulation of alternative splicing by local histone modifications: potential roles for RNA-guided mechanisms. Nucleic Acids Res 42(2), 701-713. doi: 10.1093/nar/ gkt875.

97. Moreau, P., Carosella, E., Teyssier, M., Prost, S., Gluckman, E., Dausset, J., et al. (1995). Soluble HLA-G molecule. An alternatively spliced HLA-G mRNA form candidate to encode it in peripheral blood mononuclear cells and human trophoblasts. Hum Immunol 43(3), 231-236.

98. Kovats, S., Main, E.K., Librach, C., Stubblebine, M., Fisher, S.J., and DeMars, R. (1990). A class I antigen, HLA-G, expressed in human trophoblasts. Science 248(4952), 220-223.

99. Dahl, M., Djurisic, S., and Hviid, T.V. (2014). The many faces of human leukocyte antigen-G: relevance to the fate of pregnancy. J Immunol Res 2014, 591489. doi: 10.1155/2014/591489. 


\section{Supplementary material}

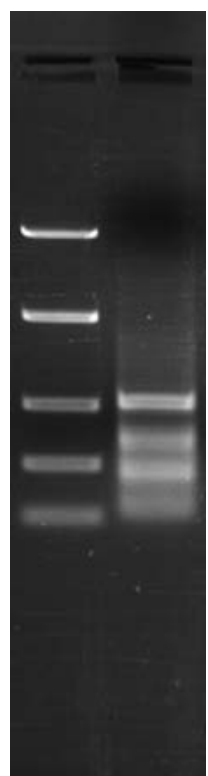

Supplementary figure S1. Visualization of alternative splicing in MamuKIR3DL20 transcripts by gel electrophoresis. In the left lane marker bands are shown (top to bottom: 5000 bp, 2000 bp, 850 bp, 400 bp, 100 bp), and in the right lane the PCR products of Mamu-KIR3DL2O are shown, using primers that were designed at the boundary of exons $1 / 2$ and at the end of exon 5. From top to bottom, the product bands correspond with the constitutively spliced MamuKIR3DL2O transcript, the Mamu-KIR2DLO5 transcript (exon 4 skipped), and the transcript that was subjected to the excision of 415 bp (exon 4 and the first 115 bp of exon 5). The lowest band shows aspecific amplification. The sequences were confirmed by Sanger sequencing.

$\longleftarrow$ Mamu-KIR3DL20

$\longleftarrow$ Mamu-KIR2DL05

$\longleftarrow$ Mamu-KIR3DL20 -415 bp 
Supplementary table 1. Eighteen different splice events were observed for human KIR. For each KIR gene for which a splice event was observed, an accession number is listed.

\begin{tabular}{|c|c|c|c|}
\hline Splice mechanism & Insertion/deletion size & Gene & Accession number \\
\hline \multirow[t]{18}{*}{ Exon skipping } & \multirow[t]{4}{*}{ Deletion 294 bp (exon 5) } & $2 D L 2$ & LS974084 \\
\hline & & $2 D L 3$ & LS974085 \\
\hline & & $2 D L 5$ & LS974086 \\
\hline & & $3 D L 1$ & LS974087 \\
\hline & \multirow[t]{6}{*}{ Deletion 51 bp (exon 6) } & $2 D L 1$ & LS974078 \\
\hline & & $2 D L 3$ & LS974079 \\
\hline & & $2 D L 4$ & LS974080 \\
\hline & & $2 D S 2$ & LS974081 \\
\hline & & $2 D S 4$ & LS974082 \\
\hline & & $3 D L 2$ & LS974083 \\
\hline & \multirow[t]{2}{*}{ Deletion 104/105 bp (exon 7) } & $2 D L 4$ & LS974091, LS974092 \\
\hline & & $3 D L 2$ & LS974090 \\
\hline & \multirow[t]{2}{*}{ Deletion 594 bp (exons 4 + 5) } & $3 D L 1$ & LS974088 \\
\hline & & $3 D L 3$ & LS974089 \\
\hline & \multirow[t]{2}{*}{ Deletion 155/156 bp (exons $6+7$ ) } & $2 D L 4$ & LS974093 ,LS974095 \\
\hline & & $3 D L 2$ & LS974094 \\
\hline & Deletion 158 bp (exons $7+8$ ) & $2 D L 4$ & LS974097 \\
\hline & Deletion 209 bp (exons $6+7+8$ ) & $2 D L 4$ & LS974096 \\
\hline \multirow[t]{3}{*}{ Alternative $3^{\prime}$ ss } & Deletion 150 bp & $2 D L 3$ & LS974098 \\
\hline & Inclusion 49 bp & $2 D L 1$ & LS974099 \\
\hline & Inclusion $170 \mathrm{bp}$ & $3 D L 1$ & LS974100 \\
\hline \multirow[t]{5}{*}{ Alternative $5^{\prime}$ ss } & Deletion 66 bp & $2 D L 4$ & LS974103 \\
\hline & Deletion 73/74 bp & $2 D L 4$ & LS974105, LS974104 \\
\hline & Deletion 198 bp & $2 D L 4$ & LS974106 \\
\hline & Inclusion 67 bp & $2 D L 4$ & LS974101 \\
\hline & Inclusion 129 bp & $3 D L 2$ & LS974102 \\
\hline \multirow[t]{4}{*}{ Alternative $3^{\prime}+5^{\prime}$ ss } & \multirow[t]{4}{*}{ Deletion 294 bp } & $2 D L 3$ & LS974108 \\
\hline & & $2 D S 4$ & LS974107 \\
\hline & & $3 D L 1$ & LS974110 \\
\hline & & $3 D L 2$ & LS974109 \\
\hline \multirow[t]{8}{*}{ Cryptic exon } & \multirow[t]{4}{*}{ Inclusion 78 bp } & $2 D L 1$ & LS974111 \\
\hline & & $2 D L 2$ & LS974112 \\
\hline & & $2 D L 3$ & LS974113 \\
\hline & & 2DS1 & LS974114 \\
\hline & \multirow[t]{4}{*}{ Inclusion 54/57 bp } & $2 D L 1$ & LS974115 \\
\hline & & 2DS1 & LS974117 \\
\hline & & $2 D S 4$ & LS974077 \\
\hline & & $2 D S 5$ & LS974116 \\
\hline
\end{tabular}


Supplementary table 2. Twenty-nine different splice events were observed for rhesus macaque KIR. For each KIR gene for which a splice event was observed, an accession number is listed.

\begin{tabular}{|c|c|c|c|}
\hline Splice mechanism & Insertion/deletion size & Gene & Accession number \\
\hline \multirow{18}{*}{ Exon skipping } & \multirow{2}{*}{ Deletion 300 bp (exon 4) } & 3DLO5 & LR030267 \\
\hline & & 3DSO5 & LR030254 \\
\hline & \multirow{6}{*}{ Deletion 294 bp (exon 5) } & 3DLO1 & LR030249, LR030461 \\
\hline & & $3 D L O 2$ & LR030261 \\
\hline & & 3DLO5 & LR030491 \\
\hline & & $3 D L 07$ & LR030266 \\
\hline & & $30 L 10$ & LR030478 \\
\hline & & $3 D L 10 A / 3 D L 02$ & LR030248 \\
\hline & Deletion 51 bp (exon 6) & $20 L 04$ & LR030486 \\
\hline & Deletion 53 bp (exon 8) & $2 D L 04$ & LR030481 \\
\hline & \multirow{2}{*}{ Deletion 594 bp (exon 4 + 5) } & $3 D L 07$ & LR030255 \\
\hline & & $3 D L 20$ & LR030240 \\
\hline & \multirow{5}{*}{ Deletion 446 bp (exon $5+6+7)$} & 3DLO1 & LR030258 \\
\hline & & 3DLO5 & LR030463 \\
\hline & & $30 L 07$ & LR030492 \\
\hline & & $30 L 10$ & LR030259 \\
\hline & & 3DL10A/3DLO2 & LR030253 \\
\hline & Deletion 115 bp (exon $6+7$ ) & $2 D L 04$ & LR030490 \\
\hline \multirow{21}{*}{ Alternative $3^{\prime}$ ss } & \multirow{13}{*}{ Deletion 36 bp } & 3DL01 & LR030242 \\
\hline & & $3 D L O 2$ & LR030495 \\
\hline & & 3DLO5 & LR030477, LR030494 \\
\hline & & $3 D L 07$ & LR030264 \\
\hline & & 3DL08 & LR030260 \\
\hline & & $3 D L 10$ & LR030466, LR030469 \\
\hline & & 3DSO1 & LR030505 \\
\hline & & 3DSO2 & LR030268 \\
\hline & & 3DSO3 & LR030503 \\
\hline & & 3DSO5 & LR030251 \\
\hline & & $3 D S w 08$ & LR030493 \\
\hline & & $3 D L 02 / 3 D L 08 A$ & LR030504 \\
\hline & & $3 D L 20$ & LR030472 \\
\hline & \multirow{5}{*}{ Deletion 216 bp } & $3 D L O 2$ & LR030496 \\
\hline & & $3 D L 08$ & LR030245 \\
\hline & & $3 D S 05$ & LR030252 \\
\hline & & 3DSw08 & LR030474 \\
\hline & & 3DL02/3DL08A & LR030243 \\
\hline & \multirow{2}{*}{ Deletion 267 bp } & 3DLO5 & LR030497 \\
\hline & & $30 S 05$ & LR030462 \\
\hline & Deletion 27 bp & $2 D L 04$ & LR030487 \\
\hline
\end{tabular}


Supplementary table 2. Continued.

\begin{tabular}{|c|c|c|c|}
\hline Splice mechanism & Insertion/deletion size & Gene & Accession number \\
\hline \multirow{16}{*}{ Alternative $3^{\prime}$ ss } & \multirow{7}{*}{ Deletion 115 bp } & $3 D L 07$ & LR030265 \\
\hline & & 3DL08 & LR030471 \\
\hline & & $3 D L 10$ & LR030479 \\
\hline & & 3DSO3 & LR030262 \\
\hline & & 3DL02/3DL08A & LR030247 \\
\hline & & 3DL10A/3DLO2 & LR030241 \\
\hline & & $3 D L 20$ & LR030464 \\
\hline & \multirow{3}{*}{ Deletion 150 bp } & 3DLO2 & LR030498 \\
\hline & & $3 D L w 03$ & LR030476 \\
\hline & & $3 D L 10$ & LR030480 \\
\hline & \multirow{2}{*}{ Deletion 176 bp } & $3 D L 07$ & LR030256 \\
\hline & & 3DL1OA/3DLO2 & LR030250 \\
\hline & Inclusion 109 bp (exon. 7) & $2 D L 04$ & LR030483 \\
\hline & Inclusion 147 bp (exon 7) & $2 D L 04$ & LR030488 \\
\hline & \multirow{2}{*}{ Inclusion 245/246 bp } & $3 D L 07$ & LR030263 \\
\hline & & 2DLO4 & LR030489 \\
\hline \multirow{4}{*}{ Alternative $5^{\prime}$ ss } & \multirow{3}{*}{ Deletion 198 bp } & 3DLO2 & LR030499 \\
\hline & & 3DSO5 & LR030467 \\
\hline & & 3DL02/3DL08A & LR030244 \\
\hline & Inclsuion 109 bp (exon 3) & 2DLO4 & LR030484 \\
\hline \multirow{7}{*}{ Alternative $3^{\prime}$ and $5^{\prime}$ ss } & Deletion 81 bp & 30502 & LR030502 \\
\hline & Deletion 107 bp & $30 S 02$ & LR030501 \\
\hline & \multirow{3}{*}{ Deletion 141 bp } & 3DL08 & LR030473 \\
\hline & & $3 D S w 08$ & LR030475 \\
\hline & & $3 D L 02 / 3 D L 08 A$ & LR030246 \\
\hline & Deletion 414 bp & $30 S 05$ & LR030500 \\
\hline & Deletion 324 bp & $3 D L 20$ & LR030468 \\
\hline Exon skipping and alt. $3^{\prime}$ ss & Deletion 415 bp & $3 D L 20$ & LR030465 \\
\hline \multirow{3}{*}{ Cryptic exon } & Inclusion 88 bp & 3DLO1 & LR030470 \\
\hline & Inclusion 147 bp (exon 5) & 2DLO4 & LR030485 \\
\hline & Inclusion 47 bp & $3 D L 07$ & LR030257 \\
\hline Intron retention & Inclusion 98/99 bp & $2 D L 04$ & LR030482 \\
\hline
\end{tabular}


Supplementary table 3. Primer sets to amplify human and macaque intron sequences. In human, two generic primer sets amplified most KIR2D genes. In macaques, different primer sets were used to amplify certain regions of different $K I R$ genes.

\begin{tabular}{|c|c|c|c|c|c|}
\hline \multicolumn{6}{|l|}{ Macaque } \\
\hline Gene & Region & Forward & Reverse & $\begin{array}{l}\text { Annealing } \\
\text { temp. }\left(C^{\circ}\right)\end{array}$ & $\begin{array}{l}\text { \# of } \\
\text { cycli }\end{array}$ \\
\hline KIR3DL & Exon 6 to exon 9 & TCACCCACTGAACCAAGCTC & АCAYGCTGGTATCTGTTGGG & 62 & 30 \\
\hline KIR3DL & Intron 4 to exon 9 & TGGGGAAGTGAGGTCAGAGA & ACACGCTGGTATCTGTTGGG & 62 & 32 \\
\hline KIR2DLO4 & Exon 5 to exon 9 & ACATTCCAGGCTGACTTCCC & GCTGTGAAGCCGGTTTTGAG & 62 & 32 \\
\hline KIR3DLO1 & Exon 5 to exon 9 & TGACCTTGTCCTGCAGTTCC & GCTGTGAAGCCGGTTTTGAG & 62 & 32 \\
\hline KIR3DL2O & Exon 1 to intron 4 & GCRTGTGTTGGGTTCTTCTTG & TCCTGTTTCTCTACCTCTGTTGGT & 62 & 32 \\
\hline
\end{tabular}

\begin{tabular}{llllll}
\hline Human & & & & \\
\hline Gene & Region & Forward & Reverse & $\begin{array}{l}\text { Annealing } \\
\text { temp. }\left(\mathbf{C}^{\circ}\right)\end{array}$ & $\begin{array}{l}\text { \# of } \\
\text { cycli }\end{array}$ \\
\hline KIR2DL & Exon 6 to exon 9 & TCACCCACTGAACCAAGCTC & GGGCAGGAGACAACTTTGGA & 60 & 32 \\
\hline KIR2DS & Exon 6 to exon 9 & TCACCCACTGAACCAAGCTC & TGCGTATGACACCTCCTGATG & 60 & 32 \\
\hline
\end{tabular}

Supplementary table 4. The Maximum Entropy Modeling Scan (MaxEntScan; MES) (68), the Position Weight Matrix (PWM) via SpliceView (69), and the Human Splice Finder (HSF) were used to predict most splicing strenght scores. If these tools failed to provide a splicing strenght score, the Weight Matrix Model (WMM) (68) and NNSplice tool (71) were used. The definition of the $3^{\prime}$ and $5^{\prime}$ splice site could differ between the different models and are listed: + determine the number of nucleotides within the exon, and- display the number of nucleotides within the intron. Also, the models define different score ranges, in which a higher value always implies a better predicted splice site. Only the HSF model provided scores for the branch point sequence (BPS), exonic splice enhancers (ESE), and exonic splice silencers (ESS).

\begin{tabular}{lllll}
\hline Software tool & 3' $^{\prime}$ ss definition & 5' ss definition & Splice strenght score range & Additional elements \\
\hline MES & -20 to +3 & +3 to -6 & -20 to +20 & - \\
\hline PWM & -9 to +4 & +3 to -6 & 0 to 100 & - \\
\hline HSF & -12 to +2 & +3 to -6 & 0 to 100 & BP, ESE, ESS \\
\hline WMM & -20 to +3 & +3 to -6 & -20 to +20 & - \\
\hline NNSplice tool & -21 to +20 & +7 to -8 & 0 to 1 & - \\
\hline
\end{tabular}





\section{Rapid characterization of complex killer cell immunoglobulin-like receptor (KIR) regions using Cas9 enrichment and Nanopore sequencing}

Jesse Bruijnesteijn ${ }^{1 *}$, Marit van der Wiel ${ }^{1}$, Natasja G. de Groot ${ }^{1}$ and Ronald E. Bontrop ${ }^{1,2}$

${ }^{1}$ Comparative Genetics and Refinement, Biomedical Primate Research Centre, Rijswijk, the Netherlands

${ }^{2}$ Theoretical Biology and Bioinformatics, Utrecht University, Utrecht, the Netherlands

*Corresponding author: Jesse Bruijnesteijn bruijnesteijn@bprc.nl Tel. nr. +31-15-2842574 Fax nr. +31-15-2842600 


\begin{abstract}
Long-read sequencing approaches have considerably improved the quality and contiguity of genome assemblies. Such platforms bear the potential to resolve even extremely complex regions, such as multigenic immune families and repetitive stretches of DNA. Deep sequencing coverage, however, is required to overcome low nucleotide accuracy, especially in regions with high homopolymer density, copy number variation, and sequence similarity, such as the MHC and KIR gene clusters of the immune system. Therefore, we have adapted a targeted enrichment protocol in combination with long-read sequencing to efficiently annotate complex $K I R$ gene regions. Using Cas9 endonuclease activity, segments of the KIR gene cluster were enriched and sequenced on an Oxford Nanopore Technologies platform. This provided sufficient coverage to accurately resolve and phase highly complex KIR haplotypes. Our strategy eliminates PCR-induced amplification errors, facilitates rapid characterization of large and complex multigenic regions, including its epigenetic footprint, and is applicable in multiple species, even in the absence of a reference genome.
\end{abstract}

\title{
Introduction
}

Repetitive regions are difficult to resolve using short-read sequencing approaches, and often remain registered for years as incomplete gaps in draft genomes (1-7). These complex stretches often involve transposable elements, microsatellites, and multicopy gene clusters, the latter of which is represented by multiple gene families that encode essential components of the immune system $(8,9)$. For example, the major histocompatibility complex (MHC) genes, known in humans as human leukocyte antigens (HLA) genes, are considered the most polymorphic gene cluster. The MHC genes co-evolved with their receptors of the killer cell immunoglobulin-like receptor (KIR) gene family, which also features striking levels of complexity $(10,11)$.

The fundamental limitations to characterizing these complex regions by short-read sequencing strategies are potentially overcome by third-generation techniques that generate high yields of long reads $(12,13)$. Oxford Nanopore sequencing may produce reads far above $100 \mathrm{~kb}$ by recording changes in the electrical current as nucleotides pass through synthetic nanopores. The data quality and throughput of nanopore sequencing is improving rapidly, and has allowed the de novo assembly of multiple human genomes (14-16). These genome assemblies contiguously span multigenic clusters, such as the $\mathrm{MHC}$ and $K I R$ gene regions, but correct annotation is hampered by the relatively low coverage, which precludes at this stage an accurate allele level resolution. Considering the important role of different multi-copy gene families in health and disease, a cost-efficient and high-resolution characterization approach regarding these types of regions is urgent.

Instead of whole genome sequencing, specific genes and regions might be enriched during library preparation. For instance, the MHC class // DRB gene region was enriched 
by long-ranged PCR, and characterized using a hybrid sequencing approach that combined Illumina and Oxford Nanopore platforms (17). Amplification steps, however, might introduce nucleotide errors during synthesis and, in addition, erase all epigenetic footprints. An amplification-free enrichment technique involves Cas9-mediated targeting of chromosome segments and nanopore sequencing (18-22). The Cas9 endonuclease activity may specifically excise genomic regions of interest that are subsequently ligated to nanopore adapters. This allows the direct sequencing of genomic segments while avoiding error prone DNA synthesis and maintaining epigenetic modifications. Efficient and specific enrichment using this approach has been demonstrated for single genes, including several cancer-related fusion genes (21), but an application for multigenic regions is absent in the literature.

In this study, we adapted the Cas9-mediated enrichment potential to resolve complex immune regions and validated this approach by the targeted characterization of KIR gene clusters in two different primate species. We focused on the KIR region in humans, which has been thoroughly characterized at the genomic level, and is important, for instance, in AIDS susceptibility and transplantation biology $(23,24)$. Rhesus macaques, however, represent a frequently used species in preclinical health research concerning, for example, COVID-19 and AIDS (25-27), but the physical location of the KIR genes is poorly understood. The KIR receptor family is involved in the regulation of NK cell activity and comprises activating and inhibitory members that may recognize particular epitopes on MHC class I molecules. A comprehensive nomenclature system distinguishes the variety of KIR receptors, and reflects the number of extracellular domains (KIR1D, KIR2D, KIR3D) and the length of the cytoplasmic tail (long, L; short, S) $(28,29)$. Subsequent numbering defines structurally similar but phylogenetically distinct genes (e.g., KIR2DL1), whereas three additional digits distinguish allotypes (e.g., KIR2DL1*001). In humans, a total of 17 KIR genes are defined, 1110 alleles of which are documented (IPD-KIR, release 2.9.0) (30).

The KIR gene repertoire is shaped by abundant tandem duplications, deletions, and chromosomal recombination events, and exceeds the plasticity of the MHC gene cluster (31-33). The KIR genes are 10 to $15 \mathrm{~kb}$ long and are arranged in a head-to-tail manner, separated by intergenic regions of approximately $2 \mathrm{~kb}$. Sequence similarity characterizes the genetic cluster, with any two KIR genes sharing $80 \%-90 \%$ homology, and allelic variants of a certain gene tend to be over $98 \%$ similar. The KIR haplotypes, defined as a segregating unit of genes located on a single chromosome, distinguish a centromeric and telomeric segment, which display diverse configurations and extensive copy number variation. The KIR genes display a variegated expression pattern, which is modulated by methylation of the promotor regions (34). For this study, we resolved six human KIR haplotypes, derived from three randomly selected human donors, at an allele-level resolution and additionally determined their methylation profiles.

The continuous evolution of the KIR gene system is reflected by the genomic diversification at a species, population, and individual level. To validate our concept, we enriched and assembled KIR haplotypes in rhesus macaques. So far, only two completely sequenced rhesus macaque KIR haplotypes have been documented, but 
previous transcriptome and segregation studies indicate extensive variation (35-39). Annotation of this complex immune region is a difficult enterprise, which is reflected by a poorly annotated KIR region in the rhesus macaque reference genome (Mmul_10) (40). Our genomic characterization of rhesus macaque KIR haplotypes demonstrated the rapid construction of complex multigenic haplotypes, even in the absence of reference sequences. Hence, adaption of this technique allows a speedy and costefficient characterization of other immune regions, from which whole genome assemblies and clinical implications might benefit.

\section{Materials and methods}

\section{Cells and genomic DNA extraction}

Human buffy coat samples from healthy donors were obtained from the Dutch blood bank (Sanquin, the Netherlands). Informed consent was obtained from all participants. Rhesus macaques with a characterized KIR transcriptome were selected from the self-sustaining colony housed at the Biomedical Primate Research Centre (BPRC) $(28,36)$. Heparin whole blood samples from these animals were obtained during annual health checks. PBMCs were isolated from human buffy coats and rhesus macaque heparin samples.

High-molecular-weight (HMW) gDNA was isolated from human and rhesus macaque PBMC samples $\left( \pm 7 \times 10^{6}\right.$ cells), using the Circulomics Nanobind CBB Big DNA Kit (Circulomics, NB-900-001-01) and following the manufacturer's instructions. The concentration and purity of the gDNA samples were determined using a Nanodrop and a Qubit platform. The (HMW) gDNA fragment length was determined by pulsed field gel electrophoresis (PFGE) in reference to a lambda PFGE ladder.

\section{Designing guiding crRNAs and constructing RNPs}

For both humans and macaques, sets of generic and specific CRISPR RNAs (crRNA) were designed within the KIR gene cluster and the flanking LILR and FCaR genes by using Benchling, a freely available online software tool (41). The crRNAs have a guiding length of $20 \mathrm{bp}$, and are designed in front of a protospacer adjacent motif (PAM) sequence "NGG", in which "N" represents any nucleotide base. The software tool provides on- and off-target scores for specific crRNAs. The on-target scores are based on optimized calculations from Doench and colleagues, with the higher score indicating the better crRNA target binding (42). The off-target scores reflect the specificity of the crRNAs, and were based on different builds of the human (NCBI36, GRCh37, GRCh38) and macaque (MMUL_1, Mmul_8.0.1) reference genomes (43). CRISPR RNAs with high off-target scores were considered specific for one KIR gene. Relatively low off-target scores (ranging from 3 to 60) were recorded for crRNAs that potentially target multiple $K I R$ genes and were included in the panel as putative generic crRNAs. In total, 54 and 45 custom crRNAs were selected to enrich the human and macaque KIR gene cluster, respectively (Sup. Table 1 and $\mathbf{2}$ ) (IDT, custom Alt-R ${ }^{\circledast}$ CRISPR-Cas9 crRNA). 
Sets of different crRNAs were pooled based on the different strand-directed orientations (sense versus anti-sense) to avoid cleavage and the sequencing of unintended short on-target fragments. The pools were defined to generate DNA fragments that comprise KIR genes from exon 1 to exon 9, or fragments that connect neighboring KIR genes (Sup. Table $\mathbf{1}$ and $\mathbf{2}$ ). The pooled crRNAs were mixed with transactivating crRNAs (tracrRNA) (IDT, \#1072534) in a 1:1 ratio and further diluted in Duplex Buffer to a final concentration of $10 \mu \mathrm{M}$. The crRNAs and tracrRNA were annealed by heating the duplex solution for $5 \mathrm{~min}$ at $95^{\circ} \mathrm{C}$, followed by cooling to room temperature (RT) on a benchtop. To subsequently construct the Cas9 ribonucleoprotein particles (RNPs), the crRNA-tracrRNA duplexes were assembled with HiFi Cas9 endonuclease (IDT, \#1081060) in 1x NEB CutSmart Buffer (NEB, \#B7204S) at a total volume of $30 \mu \mathrm{l}$ by incubating the solution for $30 \mathrm{~min}$ at RT. The Cas9 RNPs were stored until use at $4^{\circ} \mathrm{C}$ for up to a week.

\section{Cas9-mediated target enrichment and Oxford Nanopore sequencing}

Throughout the protocol, unintended fragmentation of gDNA will decrease the capturing efficiency and enrich off-target fragments. Therefore, samples should be handled with care and processed with wide-bore pipette tips. Input gDNA (5-10 $\mu \mathrm{g}$ ) was resuspended in 10x NEB CutSmart Buffer (8:1) and dephosphorylated by incubation with Quick calf intestinal phosphatase (CIP) (NEB, \# M0525S) at $37^{\circ} \mathrm{C}$ for $20 \mathrm{~min}$, followed by heating at $80^{\circ} \mathrm{C}$ for 2 min to deactivate the enzyme (Figure 1). After the sample returned to room temperature, the dephosphorylated gDNA (30 $\mu$ ) was gently mixed with a Cas9 RNP pool $(10 \mu \mathrm{l}), 10 \mathrm{mM}$ dATP $(1 \mu \mathrm{l})$, and Taq polymerase $(1 \mu \mathrm{l})$, followed by incubation at $37 \mathrm{C}^{\circ}$ for $60 \mathrm{~min}$, then $72^{\circ} \mathrm{C}$ for $5 \mathrm{~min}$, and hold at $4^{\circ} \mathrm{C}$. Ligation buffer $(20 \mu \mathrm{l})$ and sequencing adaptors $(5 \mu \mathrm{l})$ from the Ligation Sequencing Kit (ONT, \#LSK109) and Quick T4 DNA Ligase (10 $\mu$ l) (NEB, M2200S) were added to the cleaved and dA-tailed gDNA sample, followed by an incubation of 60 min at RT. Adapter-ligated samples from the same individual, which were treated with different crRNA sets, were pooled, and diluted (1:1) in TE buffer. The excess of adaptors and short DNA fragments were removed using 0.3x AMPure XP Beads (Beckman Coulter, \#A63881), which were washed twice on a magnetic rack with Long Fragment Buffer (ONT, \#LSK109). The beads were eluted in $15 \mu$ l Elution Buffer (ONT, \#LSK109). A sequencing library was prepared by adding $37.5 \mu \mathrm{l}$ Sequencing Buffer and $25.5 \mu \mathrm{l}$ Loading Beads (ONT, \#LSK109) to the processed DNA sample. Eluted samples were sequenced on a R9.4.1 flowcell using an Oxford Nanopore MinION device. Prior to sequencing, the flowcells were primed according to the manufacturer's instructions using the Flow Cell Priming Expansion Pack (ONT, \#LSK109). After 24 hours of sequencing, flowcells with over 500 active pores remaining were washed and reloaded with a second enriched library of the same gDNA sample according to the manufacturer's instructions using the Flow Cell Wash Kit (ONT, \#EXP-WSH0O3). 
HMW gDNA

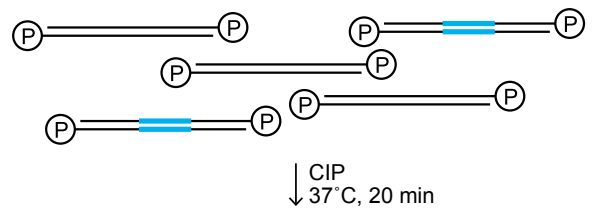

\section{Dephosphorylated} HMW gDNA

\section{Target-cleaved HMW gDNA}
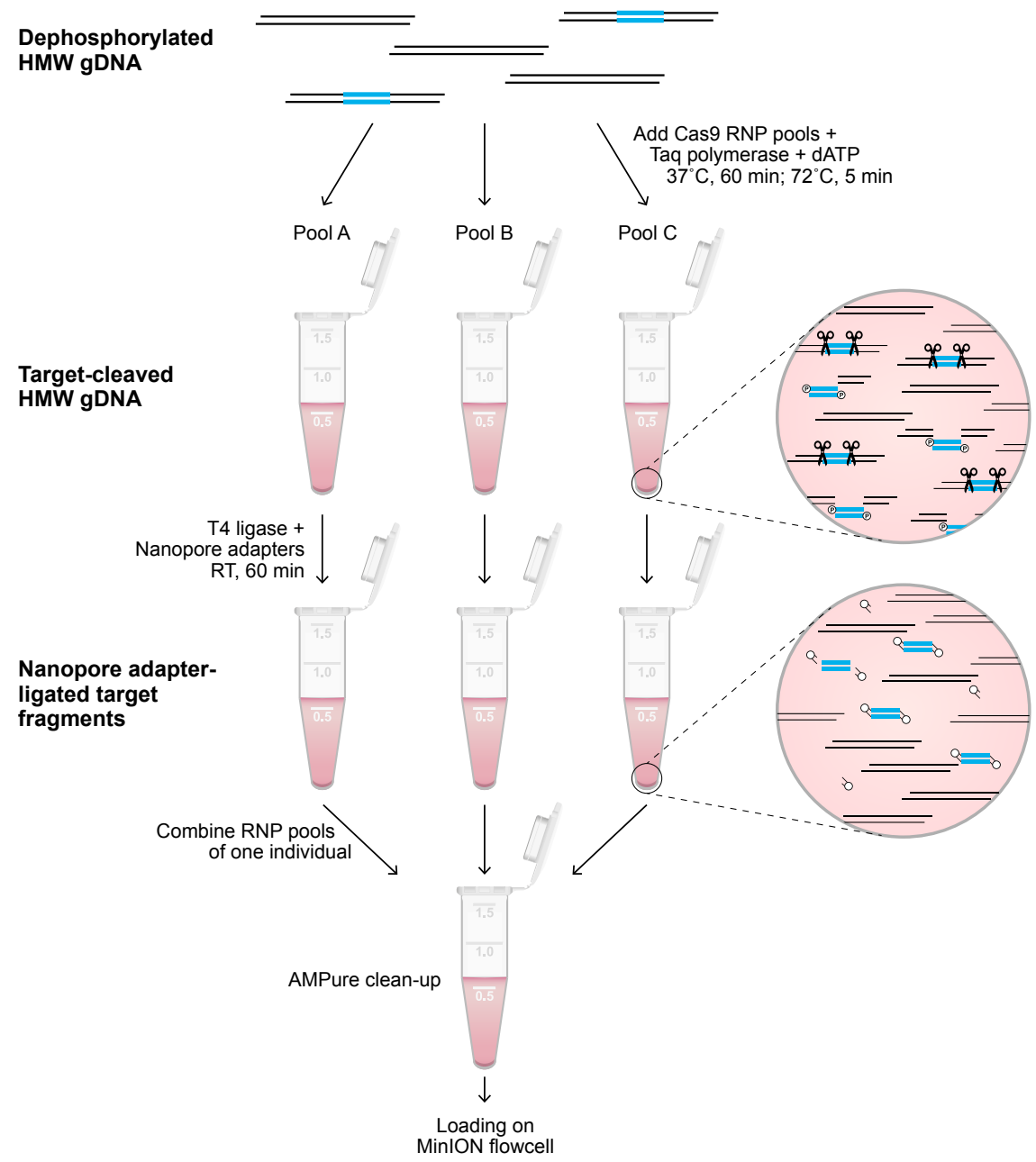

Figure 1. Library preparation of the targeted enrichment protocol. A schematic overview of the library preparation to enrich a target region (blue). Freshly isolated HMW genomic DNA was dephosphorylated by incubation with CIP for 20 min at $37^{\circ} \mathrm{C}$. Subsequently, selected pools of RNPs (Sup. Table 1 and 2 ) were added to aliquots of the dephosphorylated gDNA, and incubated for $60 \mathrm{~min}$ at $37^{\circ} \mathrm{C}$ in the presence of Taq polymerase and dATP, followed by incubation for $5 \mathrm{~min}$ at $72^{\circ} \mathrm{C}$. The available phosphate groups at the terminus of the targeted region were ligated to Nanopore adaptors during incubation with T4 ligase for 60 min at room temperature (RT). The adapter-ligated aliquots were pooled, followed by clean-up with 0.3X AMPure beads. The eluted sample was loaded on a flowcell for Nanopore sequencing. 


\section{Sequence data analysis}

Base calling and read quality assessment (min qscore 7) were performed using Guppy V3.4.1 software on a Linux platform that utilized a GeForce RTX 2080 Ti graphics processing unit (GPU). Base called reads were imported into Geneious Prime software (v.2020.1.2) for further analysis. Exon libraries, including sequences of exons 3, 4, and 7 from the human and rhesus macaque KIR databases (IPD-KIR, release 2.9.0; IPDNHKIR, release 1.3.0.0), were used as references to map the Nanopore reads into contigs based on similarity. Each on-target contig resembled a specific KIR gene or a pair of neighboring KIR genes.

For each human individual, the haplotype configurations (e.g., cA01-tA01) could be largely deduced from the read contigs generated by exon library mapping. On the basis of this knowledge, the reads of a single flowcell were re-mapped to the complete human reference genome (HG38), complemented with the particular KIR haplotype configuration references, using minimap2 (version 2.17). Consensus sequences that covered KIR genes from start to end, or that comprised segments of neighboring KIR genes, were generated from the alignments based on $65 \%$ nucleotide similarity. The consensus accuracy was determined by comparison to the reference haplotype. The on-target coverage was resolved for each individual by mapping all reads with a read length above 7,000 bp to the human reference genome (HG38) using minimap2 in Geneious software. The enrichment factor was determined as the ratio of on- and offtarget mean coverage, and reflects the enrichment efficiency.

For each rhesus macaque sample, reads from the on-target exon contigs were remapped to the contig consensus sequence using minimap2. Additional re-mapping steps were performed using the generated consensus sequences to optimize accuracy and diminish homopolymer errors. To further optimize these consensus sequences, shorter on-target reads $(<7,000 \mathrm{bp})$ were included for consensus calculations. The eventual consensus sequences were generated based on $65 \%$ nucleotide similarity. The consensus accuracy was estimated by the alignment with exon references available from previous KIR transcriptome studies (IPD-NHKIR, release 1.3.0.0). On-target coverage was defined by mapping all reads with a length of 7,000 bp or more to the rhesus macaque reference genome (Mmul_10), which was complemented with the appropriate assembled KIR haplotypes, using minimap2 in Geneious software.

\section{DNA modification profiles}

Raw signal data including information on DNA modifications, such as methylation, were processed using Guppy V4.0.12 software with the dna_r9.4.1_450bps_ modbases_dam-dcm-cpg_hac.cfg configuration and fast5_out flag. Multi-read fast5 files were converted to single-read files using multi_to_single_fast5 provided by ONT. The base called reads were mapped to a reference genome using minimap2, and subsequently called for modifications using Nanopolish (44). The methylation likelihood and frequencies were visualized by Methplotlib (45)d, using an annotated reference genome. When the reference genome did not contain the appropriate KIR genes, like 
Mmul_10 for all macaque individuals, methylation calls were annotated using a modified reference genome, which was complemented with the newly assembled haplotypes as artificial chromosomes.

\section{Results}

\section{A 'tiling' approach to enrich complex immune clusters without amplification}

The characterization of large and repetitive immune regions requires the generation and sequencing of genomic DNA (gDNA) fragments that share overlapping segments. Allelic variation in these overlaps allows the phasing of haplotypes. To achieve this goal, dephosphorylated high molecular weight (HMW) gDNA needs to be cleaved, using sets of CRISPR RNAs (crRNA) in complex with Cas9 endonuclease (Figure 1). These crRNAs are designed to target conserved stretches that are shared by members of a multigenic family. This approach will allow generic enrichment. Only at the terminus of the cleaved target sites is a phosphate group available, which is utilized for $\mathrm{dA}$-tailing and subsequent ligation to Nanopore sequencing adaptors. This 'tiling' approach facilitates the selective enrichment of large overlapping DNA-segments, and allows the subsequent sequencing of polymorphic and multigenic immune regions without the need for amplification.

\section{Enrichment of complex KIR regions}

To validate our approach, the KIR gene regions in humans and rhesus macaques were enriched and characterized (Figure 2 ). The nature of $K I R$ gene complexity required the design of species-specific sets of crRNAs to enrich complete genomic clusters (Figure 3; Sup. Table 1 \& 2). In humans, the presence of four framework genes with distinctive physical locations marks the centromeric (KIR3DL3 and KIR3DP1) and telomeric (KIR2DL4 and KIR3DL2) haplotype segments (Figure 2). Expansion and contraction of both regions resulted in haplotypes that contain 9 to 14 KIR genes, including two pseudogenes (KIR2DP1 and KIR3DP1). Important to note, however, is that some genes that are present on a given KIR haplotype may be absent from another. Human KIR haplotypes can be roughly categorized based on their gene content, into those with more an inhibitory (group A) or an activating (group B) gene profile (46). Recombination events, possibly owing to the high transposon density, might rearrange haplotype organizations (Figure 2). To determine the high content variability of this genetic cluster, 35 generic crRNAs were designed to target the differential presence of human KIR genes that may be encountered on a haplotype, whereas 12 crRNA were specific for one particular framework gene (Figure 3A; Sup. Table 1). In addition, seven crRNA were included to target the genes that flank the KIR gene cluster (LILR and $F(A R)$, in order to define both ends of the KIR haplotype.

The KIR haplotypes in rhesus macaques display even more content diversity, with 4 to $17 \mathrm{KIR}$ transcripts encoded as defined by segregation studies $(35,36)$. The only framework gene present on all haplotypes is KIR3DL20, which marks the centromeric 


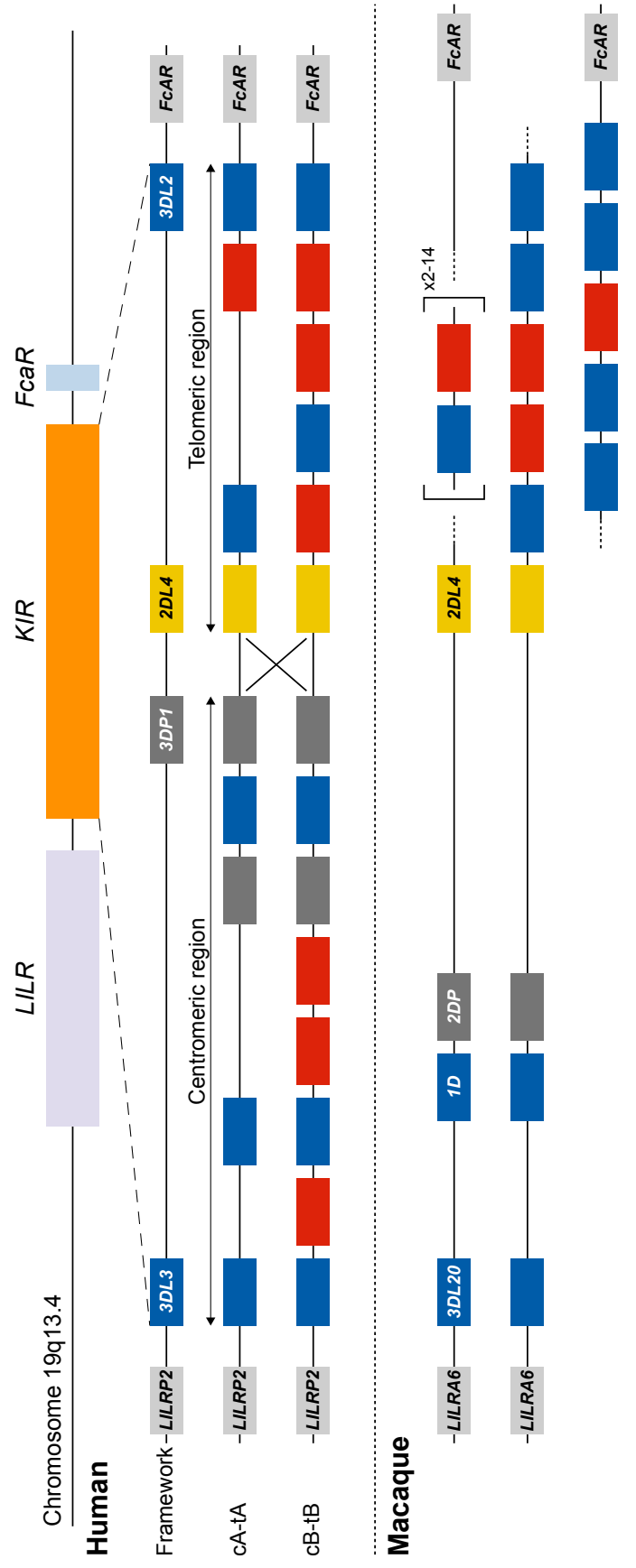

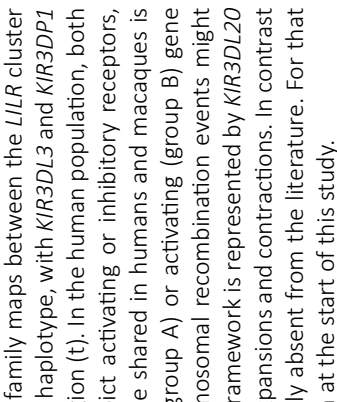

ब

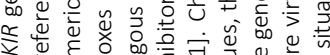

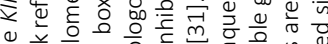

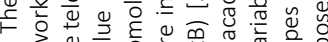

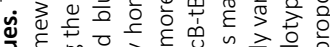
함 ฮั

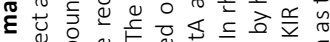

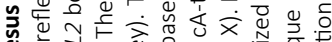
dั0

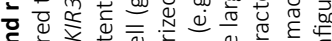

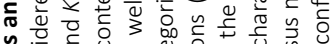

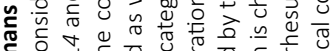

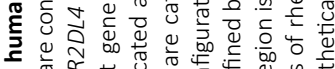
. น 完

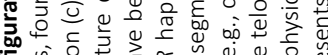

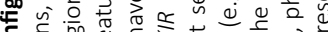

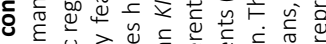

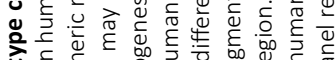

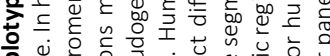

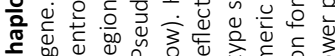

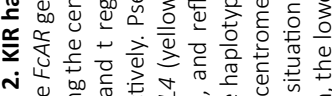

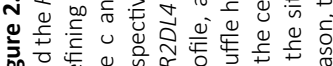

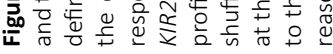



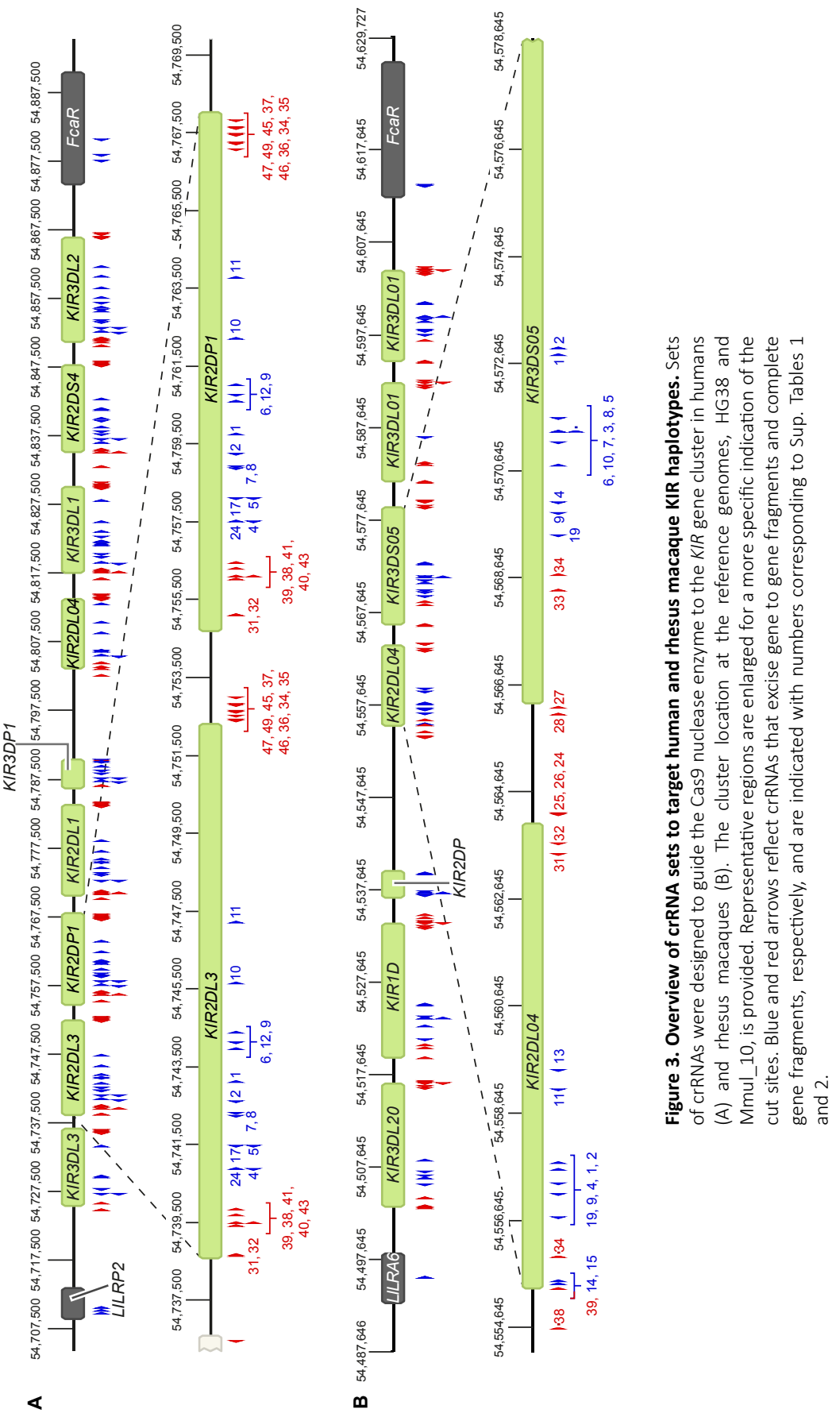
region (Figure 2). Only two other KIR genes are differentially located within this haplotype segment (KIR1D and KIR2DP). The only macaque KIR ortholog that is shared with humans, KIR2DL04, is present on approximately $80 \%$ of the telomeric haplotype segments. This haplotype region is further characterized by a differential number of $K I R$ genes in highly diverse frequencies. To enrich the macaque KIR cluster, we designed 24 generic crRNAs that target the variable gene tandem, and complemented those with crRNAs specific for KIR1D, KIR2DLO4, and KIR3DL20, and the flanking genes, LILRA6 and FCAR (Figure 3B; Sup. Table 2).

\section{Assembly of human KIR haplotypes}

For this communication, the human target region was defined at the start of exon 5 of LILRP2 to the end of exon 1 of FCAR, thereby comprising the complete KIR haplotype (Figure 2). On the human reference genome (HG38), this target region spans approximately $161 \mathrm{~kb}$ and contains nine KIR genes, one of which encodes an activating KIR (group A haplotype). To account for the variability of KIR haplotype configurations, four additional reference sequences, which were assembled by Fosmid sequencing and reflected different $K I R$ genotypes, were included in our panel (47). In total, five reference KIR regions were used to assemble captured reads and to determine sequence accuracy.

For each of the three randomly selected human individuals, two Nanopore flowcells were loaded with HMW gDNA samples that were enriched either for fragments that comprise a complete $K I R$ gene or fragments that connect and distinguish neighboring $K I R$ genes. An average of 1.26 million Nanopore reads were yielded per flowcell (Table 1). Of these reads, $4.2 \%$ to $19.3 \%$ had a length of 7,000 bp or longer, which is required to connect and distinguish neighboring $K I R$ genes. The percentage of sizeselected reads that mapped to the target region ranged from $1.6 \%$ to $2.5 \%$ ( $\pm 4,015$ reads), which provided a median coverage ranging from 269 to $323 \mathrm{X}$. The enrichment factor, which reflects the efficiency of the targeted enrichment, ranged from 215 to $394 X$. The length of the consensus sequences that were generated from the on-target reads ranged from 6.5 to $29.7 \mathrm{~kb}$, and their assembly covered complete reference KIR haplotype configurations (Figure $\mathbf{4 A}, \mathbf{4 B}$, and $\mathbf{4 C}$ ). The consensus accuracy compared to the reference sequences ranged from $96.7 \%$ to $99.9 \%$.

The centromeric and telomeric regions were completely assembled and grouped into four different segment configurations (cA01, cB02, tA01, tB01). Phasing of complete KIR haplotypes was achieved in two individuals, including a homozygous haplotype configuration using allele level resolution (Figure 4A, 4B and $4 D$ ). This result demonstrates the resolution power of our approach. For another homozygous setting, KIR haplotypes were phased for all genes, except for KIR2DL1 (Figure 4C). This gene was identical on both haplotypes, and defined a SNP desert ( $25 \mathrm{~kb})$ that hampered complete phasing (Figure S1). The largest completely assembled and phased haplotype comprised 11 KIR genes and covered a total of approximately 176 kb (Figure 4B). 
Table 1. Overview of the total reads, read length, on-target hits, and the coverage of the target region in human samples.

\begin{tabular}{|c|c|c|c|}
\hline & \multicolumn{3}{|c|}{ Gene fragments (flowcell 1) } \\
\hline & $\# 1$ & \#2 & \#3 \\
\hline Total \# reads & 789705 & 1175600 & 1413919 \\
\hline Total \# reads > $7.000 \mathrm{bp}$ & 57830 & 49535 & 59575 \\
\hline Percentage reads $>7.000 \mathrm{bp}$ & 7.3 & 4.2 & 4.2 \\
\hline Average read length (bp) & 2603 & 2114 & 2092 \\
\hline \multirow[t]{3}{*}{ Average read length $(\mathrm{bp})$ of reads $>7.000 \mathrm{bp}$} & 13410 & 10895 & 13317 \\
\hline & \multicolumn{3}{|c|}{ Gene to gene fragments (flowcell 2) } \\
\hline & $\# 1$ & $\# 2$ & $\# 3$ \\
\hline Total \# reads & 1060000 & 1078502 & 2012903 \\
\hline Total \# reads > $7.000 \mathrm{bp}$ & 204797 & 152372 & 108688 \\
\hline Percentage reads > $7.000 \mathrm{bp}(\%)$ & 19.3 & 14.1 & 5.4 \\
\hline Average read length (bp) & 5062 & 3728 & 2021 \\
\hline \multirow[t]{3}{*}{ Average read length $(\mathrm{bp})$ of reads $>7.000 \mathrm{bp}$} & 17206 & 13327 & 13455 \\
\hline & \multicolumn{3}{|c|}{ Total reads (flowcells 1 and 2) } \\
\hline & $\# 1$ & $\# 2$ & \#3 \\
\hline On-target reads (> 7.000 bp reads) & 4470 & 3308 & 4268 \\
\hline Percentage on-target reads & 1.7 & 1.6 & 2.5 \\
\hline Mean coverage target region & 323 & 269 & 315 \\
\hline Enrichment factor & 215 & 299 & 394 \\
\hline
\end{tabular}

\section{Assembly of rhesus macaque KIR haplotypes without reference genome}

The successful deciphering of highly variable rhesus macaque KIR haplotypes validated our enrichment and characterization approach further, even in the absence of genomic reference sequences. The haplotype content was initially determined by previous transcriptome and segregation studies $(35,36)$, but the physical location of $K I R$ genes remained elusive. The target region was defined at the start of exon 7 in LILRA6 to the end of exon 2 in FCAR, thereby comprising the complete rhesus macaque $K I R$ cluster. This region covered $330 \mathrm{~kb}$ on the rhesus macaque reference genome (Mmul_10). Ironically, the KIR gene cluster is poorly assembled and annotated on this reference genome and is at present not suitable as a reference to assemble $K I R$ haplotypes.

The rhesus macaque KIR cluster was enriched from samples of three animals. One or two flowcells were used to enrich fragments that comprised KIR genes from start to end, or connected neighboring KIR genes. A range of $2.5 \%$ to $36.3 \%$ of the total reads had a read-length of 7,000 bp or longer (Table 2). The percentage captured on-target reads ranged from $0.5 \%$ to $4.2 \%$ (Table 2 ). The mean coverage of the target region was determined by mapping all reads to the reference genome (Mmul_10), which was complemented with the assembled KIR haplotypes as artificial chromosomes and reached $26 \mathrm{X}$ to $91 \mathrm{X}$. The enrichment factor ranged from $128 \mathrm{X}$ to $637 \mathrm{X}$. Consensus 
Table 2. Overview of the total reads, read length, on-target hits, and the coverage of the target region in rhesus macaque samples. *For \#3, a single flowcell was used for all different crRNA pools, generating fragments containing a KIR gene from start to end, and fragments that span from one KIR gene to another.

\begin{tabular}{|c|c|c|c|}
\hline & \multicolumn{3}{|c|}{ Gene fragments (flowcell 1) } \\
\hline & $\# 1$ & $\# 2$ & $\# 3$ \\
\hline Total reads & 252981 & 453649 & 2208000 \\
\hline Total reads $>7.000 \mathrm{bp}$ & 25062 & 54772 & 55490 \\
\hline Percentage reads $>7.000 \mathrm{bp}$ & 9.9 & 12.1 & 2.5 \\
\hline Average read length (bp) & 3098 & 3375 & 1978 \\
\hline \multirow[t]{3}{*}{ Average read length $(\mathrm{bp}$ ) of reads $>7.000 \mathrm{bp}$} & 11258 & 11875 & 9319 \\
\hline & \multicolumn{3}{|c|}{ Gene to gene fragments (flowcell 2) } \\
\hline & $\# 1$ & $\# 2$ & - \\
\hline Total reads & 48586 & 20956 & - \\
\hline Total reads $>7.000 \mathrm{bp}$ & 5179 & 7607 & - \\
\hline Percentage reads > $7.000 \mathrm{bp}(\%)$ & 10.7 & 36.3 & - \\
\hline Average read length (bp) & 2884 & 7110 & - \\
\hline \multirow[t]{3}{*}{ Average read length (bp) of reads $>7.000 \mathrm{bp}$} & 12226 & 14789 & - \\
\hline & \multicolumn{3}{|c|}{ Total reads (flowcells 1 and 2 ) } \\
\hline & $\# 1$ & $\# 2$ & $\# 3$ \\
\hline On-target reads (> 7.000 bp reads) & 1070 & 1005 & 292 \\
\hline Percentage on-target reads & 4.3 & 1.8 & 0.5 \\
\hline Mean coverage target region & 60.8 & 91.8 & 26.8 \\
\hline Enrichment factor & 637 & 404 & 128 \\
\hline
\end{tabular}

sequences were generated, which displayed lengths ranging from 7.2 to $20.0 \mathrm{~kb}$. The accuracy of the consensus sequences is estimated by a comparison with rhesus macaque exon sequences extracted from the non-human KIR Database (IPD-NHKIR, release 1.3 .0 .0 ), and reached $96.7 \%$ to $100 \%$ similarity at these coding regions.

An allele level resolution allowed the phasing of six rhesus macaque KIR haplotypes (Figure 5). Even with a single flowcell, sufficient coverage was reached to define haplotypes at an allele level resolution (Figure $\mathbf{5 C}$ ). The largest haplotype contained 16 KIR genes and spanned $280 \mathrm{~kb}(\mathrm{H} 15)$. The different allelic copies of KIR3DLO1, KIR3DLO7 and KIR3DSO1 that could be distinguished on this extended haplotype suggests its generation by multiple chromosomal recombination events. The shortest KIR haplotype encoded five KIR gene members ( $\mathrm{H} 10)$. A fusion gene, which consists of segments from two distinct KIR genes, and that are occasionally generated by chromosomal recombination events, was identified on $\mathrm{H} 14$ (KIR3DL20*O3OR) (Figure 5A). These recombined entities often remain undetected by current genotyping approaches.

\section{Methylation profile of the multigenic KIR region}

Amplification-free enrichment and nanopore sequencing allow the characterization of DNA modification profiles. KIR genes display a variegated expression on NK cells and subsets of $T$ cells, which is tightly regulated by methylation of the promotor region 
A

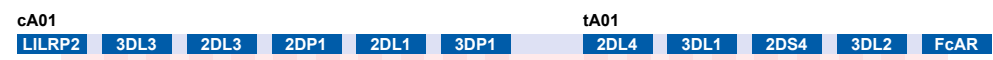

$\frac{10,8 \mathrm{~kb}}{99,2 \%}\left|\frac{13,0 \mathrm{~kb}}{99,6 \%}\right| \frac{11,4 \mathrm{~kb}}{99,7 \%}\left|\frac{12,8 \mathrm{~kb}}{99,9 \%}\right| \frac{6,5 \mathrm{~kb}}{\mid 99,5 \%}\left|\quad \frac{14,8 \mathrm{~kb}}{99,4 \%}\right| \frac{12,4 \mathrm{~kb}}{99,1 \%}\left|\frac{14,8 \mathrm{~kb}}{\mid 99,3 \%}\right| \frac{15,3 \mathrm{~kb}}{99,9 \%}$

(1) $\frac{12,5 \mathrm{~kb}}{98,8 \%}\left|\frac{14,3 \mathrm{~kb}}{99,4 \%}\right| \frac{13,0 \mathrm{~kb}}{99,8 \%}\left|\frac{12,9 \mathrm{~kb}}{99,8 \%}\right| \frac{11,7 \mathrm{~kb}}{99,7 \%}\left|\frac{16,0 \mathrm{~kb}}{99,6 \%}\right| \frac{15,1 \mathrm{~kb}}{99,8 \%}\left|\frac{14,2 \mathrm{~kb}}{99,8 \%}\right| \frac{12,0 \mathrm{~kb}}{99,8 \%}\left|\frac{17,8 \mathrm{~kb}}{99,7 \%}\right|$

$\frac{14,0 \mathrm{~kb}}{98,9 \%}\left|\frac{14,3 \mathrm{~kb}}{99,3 \%}\right| \frac{13,6 \mathrm{~kb}}{99,3 \%}\left|\frac{13,6 \mathrm{~kb}}{99,5 \%}\right| \frac{10,7 \mathrm{~kb}}{99,5 \%}\left|\frac{17,1 \mathrm{~kb}}{99,6 \%}\right| \frac{13,5 \mathrm{~kb}}{99,6 \%}\left|\frac{14,4 \mathrm{~kb}}{97,6 \%}\right| \frac{12,1 \mathrm{~kb}}{99,4 \%}\left|\frac{17,8 \mathrm{~kb}}{99,6 \%}\right|$

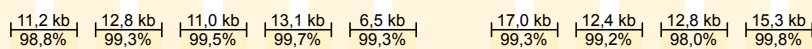
\begin{tabular}{l|l|l|l|l|l|l|l|l|l|l|l} 
LLILR2 & 3DL3 & 2DL3 & 2DP1 & 2DL1 & 3DP1 & 2DL4 & 3DL1 & 2DS4 & 3DL2 & FcAR \\
cA01 & & & &
\end{tabular}

B CA01 tB01 \begin{tabular}{|l|l|l|l|l|l|l|l|l|l|l|}
\hline $3 D L 3$ & $2 D L 3$ & $2 D P 1$ & $2 D L 1$ & $3 D P 1$ & $2 D L 4$ & $3 D S 1$ & $2 D L 5$ & $2 D S 5$ & $2 D S 1$ & $3 D L 2$ \\
\hline
\end{tabular}

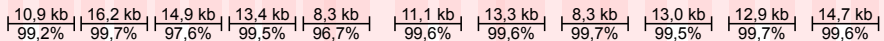

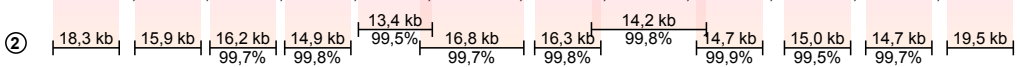

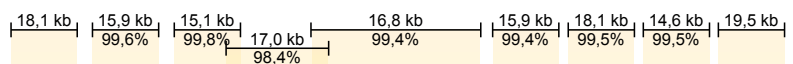

$\frac{11,0 \mathrm{~kb}}{99,3 \%}+\frac{13,0 \mathrm{~kb}}{99,6 \%}+\frac{12,9 \mathrm{~kb}}{99,6 \%}+\frac{13,2 \mathrm{~kb}}{98,2 \%}\left|\frac{11,2 \mathrm{~kb}}{99,2 \%}+\frac{15,2 \mathrm{~kb}}{99,4 \%}\right| \frac{12,9 \mathrm{~kb}}{\frac{99,3 \%}{1}} \mid \frac{14,5 \mathrm{~kb}}{99,3 \%}$

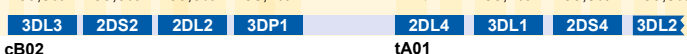

C \begin{tabular}{l|l|l|l|l|l|l|l|l|l|l} 
CA01 & & \multicolumn{1}{l}{ tA01 } & & & \\
LILRP2 & 3DL3 & 2DL3 & 2DP1 & 2DL1 & 3DP1 & 2DL4 & 3DL1 & 2DS4 & 3DL2 & FCAR
\end{tabular}

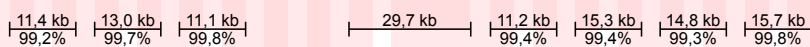

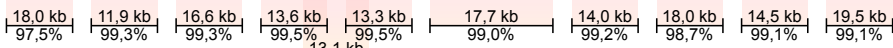

(3) $\frac{21,5 \mathrm{~kb}}{99,3 \%}\left|\frac{12,9 \mathrm{~kb}}{99,2 \%}\right| \frac{15,0 \mathrm{~kb}}{99,3 \%}\left|\frac{14,4 \mathrm{~kb}, 7 \%}{99,6 \%}\right| \frac{13,3 \mathrm{~kb}}{99,6 \%}\left|\frac{17,0 \mathrm{~kb}}{99,2 \%}\right| \frac{14,0 \mathrm{~kb}}{99,2 \%}\left|\frac{17,6 \mathrm{~kb}}{97,4 \%}\right| \frac{17,6 \mathrm{~kb}}{99,2 \%} \mid \frac{17,7 \mathrm{~kb}}{199,4 \%}$

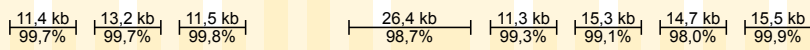

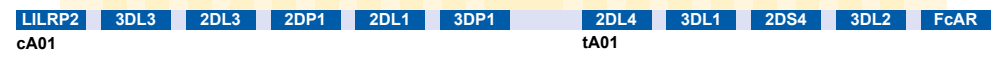

cA01-tA0

3DL3*002
like $2 D L 3 * 001-2 D P 1 * 002$
like $2 D L 1 * 003-3 D P 1 * 010-2 D L 4 * 008-3 D L 1 * 001-2 D S 4^{*} 003-3 D L 2 * 001$

(1)

$3 D L 3^{*} 001-2 D L 3 * 002-2 D P 1 * 003-2 D L 1 * 003-3 D P 1 * 006-2 D L 4 * 008-3 D L 1^{*} 004-2 D S 4^{*} 006-3 D L 2 * 009$ CA01-tA01

cA01-tB01 3DL3*010-2DL3*001-2DP1*002-2DL1*003-3DP1*006-2DL4*005-3DS1*013-2DL5*001 2DS5*002-2DS1*002-3DL2*007

(2) $3 D L 3^{*} 003-2 D S 2^{*} 001-2 D L 2^{*} 003-3 D P 1^{*} 001-2 D L 4^{*} 001-3 D L 1^{*} 002-2 D S 4^{*} 0013 D L 2^{*} 002$ cB02-tA01

CA01-tA01

(3) 3DL2*002-2DL3*001-2DP1*002 $3 D P 1^{*} 003-2 D L 4^{*} 011-3 D L 1^{*} 005-2 D S 4^{*} 010-3 D L 2^{*} 001$ $3 D L 2 * 010-2 D L 3^{*} 001-2 D P 1^{*} 002 \int_{3 D P 1 * 010-2 D L 4 * 008-3 D L 1} 004-2 D S 4^{*} 006-3 D L 2 *$ like cA01-tA01 
(34). Therefore, we have investigated whether we could determine methylation signatures for the KIR gene region. The modification likelihood (low, blue; high, red) was defined for all positions on individual reads that mapped to the KIR cluster (Figure $\mathbf{A}$ and $\mathbf{B}$, top part). Based on up to 100 reads, the frequency of modification was determined for different KIR gene promotor regions (Figure 6A and $\mathbf{B}$, black line at the bottom part). A high modification likelihood and frequency was determined for the promotor region of all KIR genes in the human samples (Figure 6A). The modification frequency of the complete intergenic region ranged from $62 \%$ to $93 \%$ and seems to slightly increase in the proximal promotor adjacent to exon 1 . The intergenic regions in the rhesus macaque KIR cluster also displayed high likelihood and frequency of modification (Figure 6B). However, the slight increase of modification frequency at the proximal promotor is not observed. Because all DNA samples were isolated from peripheral blood lymphocytes, the high methylation frequency is in line with scare expression of KIR on most prominent lymphocytes. The true resolution power of this approach would emerge using isolated cell populations or single-cell clones.

\section{Discussion}

Most multigenic regions are subjected to complex evolutionary processes, in which species-specific gene duplications, deletions, and recombination events shape a genetic cluster. Immune gene families, such as KIR and MHC, diversified under selective pressure by a continuous arms race with pathogens, and individuals might benefit from extensive variation at the population level (48). The high gene content diversity and sequence similarity challenges rapid genomic characterization of these multigenic families.

We make available a rapid enrichment and characterization approach for complex immune regions, which overcomes many of the limitations of short-read or wholegenome sequencing. The Oxford Nanopore MinION sequencing device and flow cells are relatively inexpensive. This makes the descirbed method widely accessible to

4 Figure 4. Enrichment and phasing of human KIR haplotypes. Human donors 1, 2, and 3 were randomly selected, and displayed one heterozygous (\#2) and two homozygous (\#1, \#3) KIR haplotype configurations. Consensus sequences (black bars) that covered KIR genes from start to end, or that comprised segments from neighbouring KIR genes, were mapped to corresponding haplotype references derived from the human reference genome (HG38) or from previous Fosmid sequencing studies (Roe et al. 2017). The consensus sequence length and accuracy are indicated. Colored columns indicate the mapped regions, in which a darker color illustrates overlapping consensus sequences. KIR haplotypes were completely phased in two individuals (A, B). The third individual shared an identical KIR2DL1 gene on both haplotypes, which located an extended SNP desert (25 kb; Figure S2) (C). KIR haplotypes of this individual were phased at all genes at an allele level resolution, except for the KIR2DL1 gene. Four differential KIR region configurations from three randomly selected donors were resolved. Based on allelic polymorphism, each of these haplotypes appears to be unique (D). In addition, three new variants of documented alleles were defined, which are indicated with "like". 


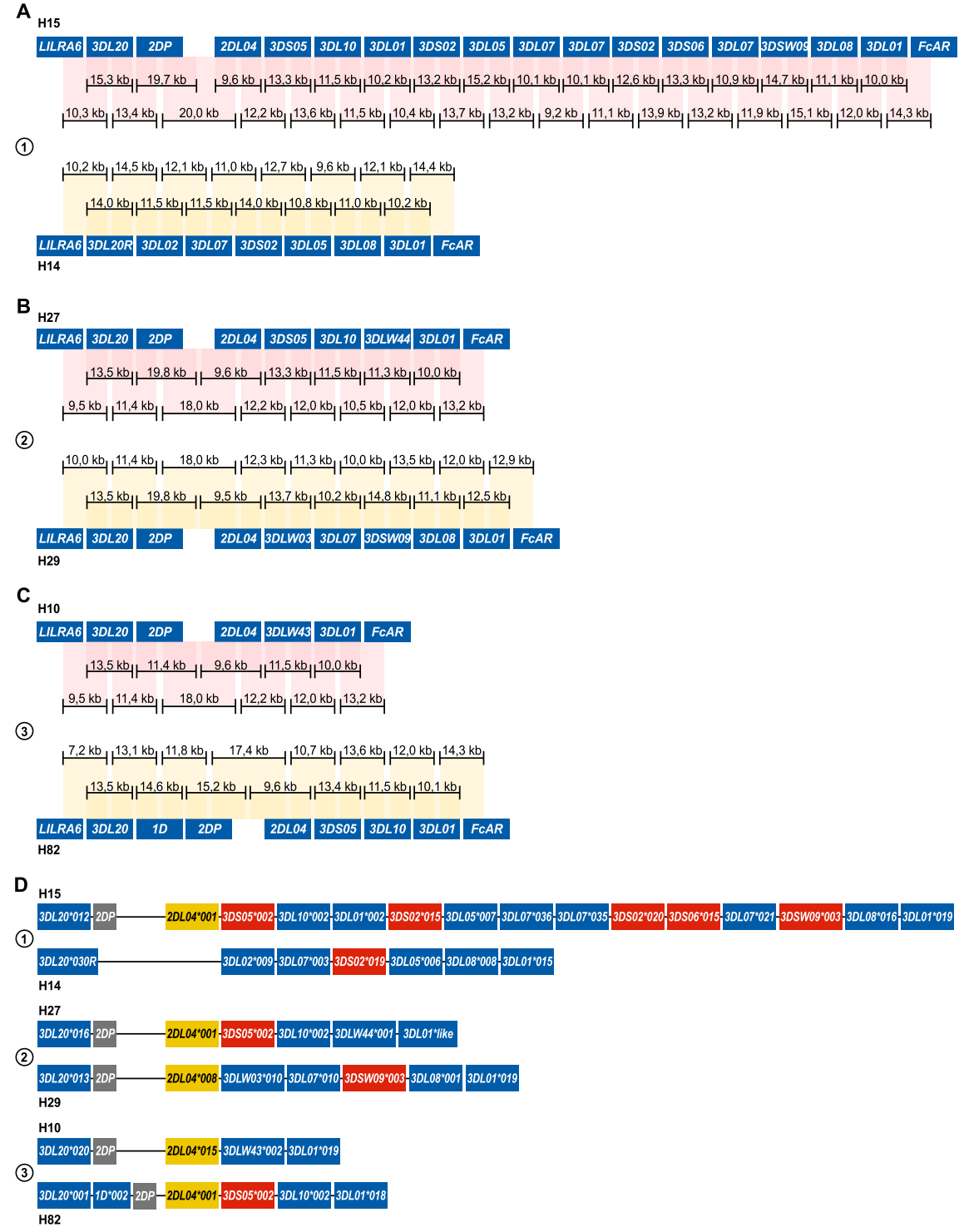

Figure 5. Enrichment and phasing of rhesus macaque $K I R$ haplotypes. For rhesus macaque samples (\#1, \#2, and \#3), the KIR gene content was determined by a combination of transcriptome and segregation studies [32-34]. Our approach resulted in an accurate annotation of the complex KIR regions present in these animals. Consensus sequences (black bars) that covered KIR genes from exon 1 to exon 9, or that comprised segments from neighboring KIR genes, were assembled. The physical order of KIR genes at the haplotype was determined by the extensive overlaps and the alignment with exon sequences from the IPD-NHKIR. The consensus sequence lengths are indicated. Phasing was achieved for all haplotypes, and reflected six different configurations (A, B, C). For each sample, haplotypes were sorted out at an allele level resolution (D). One new allele was defined for KIR3DLO1 (H27), which is indicated with "like". 
laboratories without the need for dedicated sequencing facilities, which contrasts other long-read sequencing platforms, such as PacBio sequencing. Extensive diversity in gene content of multigene families was resolved by targeting long overlapping genomic fragments that covered genes from start to end, or that contained segments of neighboring genes. These enriched fragments display a median length of approximately $10 \mathrm{~kb}$ with outliers up to $25 \mathrm{~kb}$, which is in line with the expected target fragments. Isolation of longer fragments might be achieved using Cas9-enrichment but was not required for this study design. Efficient targeting was based on using generic crRNAs, which yielded a relatively high median coverage at the region of interest and allowed the generation of accurate consensus sequences. Most inaccuracies involved homopolymers, here defined as a stretch of three or more identical bases, which simultaneously impact the current measured by the nanopore. The consensus sequence accuracy was, however, sufficient to define alleles and to phase complex KIR haplotypes. Only at large identical stretches in which overlapping fragments lack allelic variation might this technique hamper the phasing of haplotypes (Figure S1). These SNP deserts might be resolved by additional crRNAs that flank the identical stretch. In addition, the continuous improvement of library preparation chemistry, base-calling algorithms, and post-sequencing correction software might eventually increase the length of enriched fragments and the accuracy of nanopore sequencing.

The target coverage was sufficient for the characterization of KIR haplotypes at the allele level resolution, but the enrichment performance displayed some deviations in the different samples (Tables $1 \& 2$ ). The variance in total read numbers and read length is likely to be affected by flowcell variations, gDNA sample quality and short read clean-up efficiency during library preparation. Despite the deviations in read counts, the on-target coverage was sufficient, even when only a single flowcell was used (Table 2, \#3; Figure 5C). Further optimalization of the enrichment protocol, by designing additional crRNAs or by enhancing the Cas9 efficiency with improved chemistry, might provide higher on-target coverage. Samples might then be multiplexed on a single flow cell to further reduce the costs of KIR cluster characterization. Nonetheless, the current approach already ensured highly accurate and overlapping consensus sequences that cost-efficiently resolved complete human and macaque KIR haplotypes at an allele level resolution (Figure 4D \& 5D).

The KIR receptors display a selective and variegated expression on the cell surface of NK cells and subsets of T cells $(49,50)$. The majority of these cells only express a single KIR gene, whereas smaller fractions might have two to four KIR receptors on their cell surface. This selective expression pattern is most likely regulated by methylation of the promotor region, which is initiated during NK cell differentiation (51). The high modification frequency of the KIR promotor regions (Figure 6) indicates low levels of expression in the characterized samples (34). Our HMW DNA samples were isolated from whole blood, which includes only a small fraction of KIR-positive cells that might display hypomethylated promotor regions. Nevertheless, the epigenetic modification of members from highly complex multigenic families could be determined using our approach. 
A

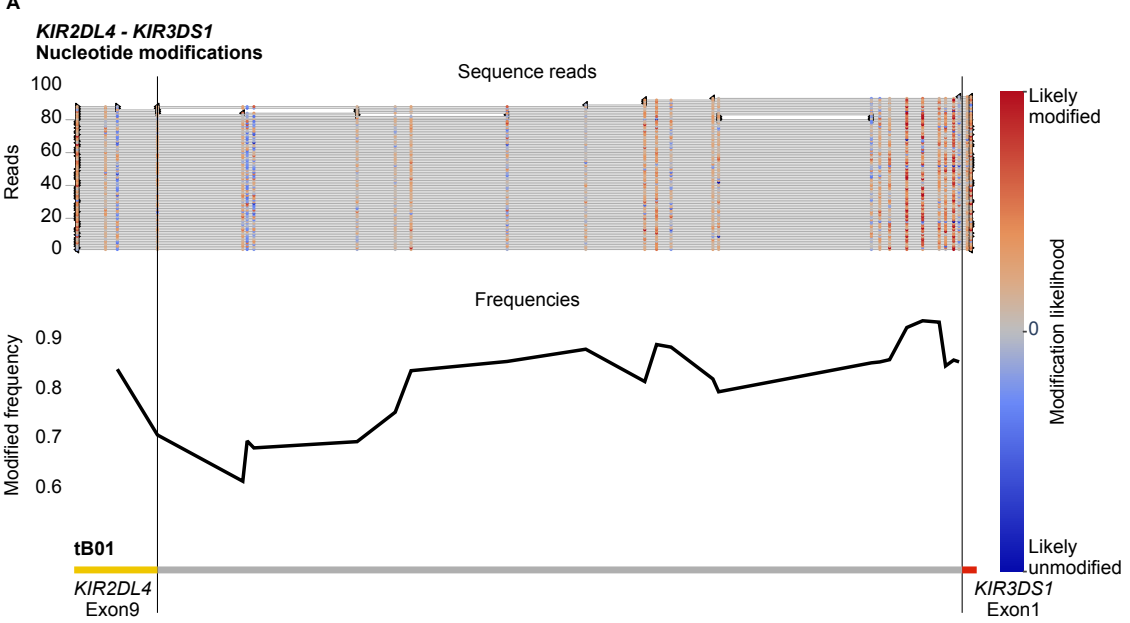

B

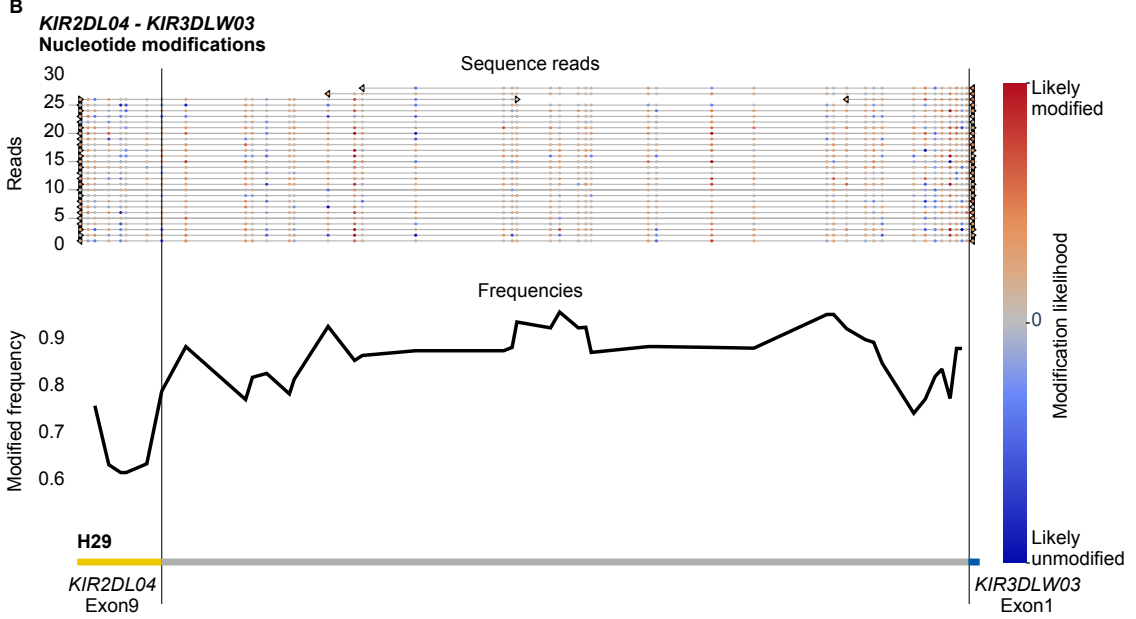

Figure 6. DNA modification profiles. DNA modification profiles are displayed for one human (sample \#2) (A) and one rhesus macaque (sample \#2) (B) KIR gene. The epigenetic predictions were calculated with up to 100 randomly selected reads per enriched KIR segment. Indicated are the modification likelihoods (red, high; blue, low), the modification frequency, which ranges from 0 to 1 , and the annotation that is based on the (complemented) reference genomes (HG38 and Mmul_10). Plots were generated by Methplotlib (De Coster et al. 2020). The KIR promotor region, which is approximately 300 bp in front of exon 1 (Chan et al. 2003), is highly modified for human KIR3DS1 (A) and macaque KIR3DLWO3 (B), with modification frequencies ranging from 85 to $95 \%$. These two regions are representative for all other $K I R$ gene regions studied in our human and rhesus macaque cohorts. 
Resolving complete multigenic haplotypes and their epigenetic profiles at the allele level resolution might provide biological and diagnostic insights into the role of these complex systems in health and disease. This is thoroughly demonstrated for different combinations of KIR and HLA that might have an impact on human health, susceptibility or resistance to disease, or may affect graft survival success in transplantation biology $(23,24,52)$. There is, however, a lack of consensus from these association studies. One of the causes that might explain these contradicting outcomes is the predominant strategy to characterize common structural motifs, such as centromeric and telomeric KIR haplotype segments, thereby simplifying the plasticity of these immune regions. Using our rapid enrichment and sequencing approach, more comprehensive associations might be defined based on completely defined haplotypes at the allele level resolution.

Members of multigenic families often encode components of essential immune responses and exhibit extensive copy number variation and allelic polymorphism. The continuous diversification and selection of these genes enables adaption to pathogens but might also generate candidates that enhance susceptibility to immune-related disorders. These adaptions might involve SNPs that are mapping in coding regions, whereas others are not, and may impact, for instance, gene (expression) regulation and alternative splicing potential. This enrichment technique fosters a cost-efficient and rapid strategy to characterize complex immune-receptor families at an allele level. Insights regarding complex immune clusters might provide a comprehensive perspective on biological interpretations, and lift SNP disease association studies from the allele to the haplotype/region level, in which all polymorphisms are considered.

\section{Acknowledgements}

We thank D. Devine for editing the manuscript and F. van Hassel for preparing the figures.

\section{Data availability}

All raw Nanopore files and processed sequencing data generated in this study have been submitted to the European Nucleotide Archive (ENA) (https://www.ebi.ac.uk/ ena/browser/home) under accession number PRJEB43311. 


\section{References}

1 Alkan, C, Cardone, MF, Catacchio, CR, Antonacci, F, O’Brien, SJ, Ryder, OA, et al., Genome-wide characterization of centromeric satellites from multiple mammalian genomes. Genome research 21 (2011) 137-45. doi: 10.1101/gr.111278.110

2 Chinwalla, AT, Cook, LL, Delehaunty, KD, Fewell, GA, Fulton, LA, Fulton, RS, et al., Initial sequencing and comparative analysis of the mouse genome. Nature 420 (2002) 520-562. doi: 10.1038/nature01262

3 Gibbs, RA, Rogers, J, Katze, MG, Bumgarner, R, Weinstock, GM, Mardis, ER, et al., Evolutionary and biomedical insights from the rhesus macaque genome. Science (New York, N.Y.) 316 (2007) 222-34. doi: 10.1126/ science. 1139247

4 International Human Genome Sequencing, C, Finishing the euchromatic sequence of the human genome. Nature 431 (2004) 931-945. doi: 10.1038/nature03001

5 Lindblad-Toh, K, Wade, CM, Mikkelsen, TS, Karlsson, EK, Jaffe, DB, Kamal, M, et al., Genome sequence, comparative analysis and haplotype structure of the domestic dog. Nature 438 (2005) 803-819. doi: 10.1038/ nature 04338

6 Marques-Bonet, T, Ryder, OA, and Eichler, EE, Sequencing primate genomes: what have we learned? Annual review of genomics and human genetics 10 (2009) 355-86. doi: 10.1146/annurev.genom.9.081307.164420

7 Waterson, RH, Lander, ES, Wilson, RK, The Chimpanzee, S, and Analysis, C, Initial sequence of the chimpanzee genome and comparison with the human genome. Nature 437 (2005) 69-87. doi: 10.1038/nature04072

8 Peona, V, Blom, MPK, Xu, L, Burri, R, Sullivan, S, Bunikis, I, et al., Identifying the causes and consequences of assembly gaps using a multiplatform genome assembly of a bird-of-paradise. bioRxiv (2019) 2019.12.19.882399. doi: 10.1101/2019.12.19.882399

9 Thomma, BPHJ, Seidl, MF, Shi-Kunne, X, Cook, DE, Bolton, MD, van Kan, JAL, et al., Mind the gap; seven reasons to close fragmented genome assemblies. Fungal Genetics and Biology 90 (2016) 24-30. doi: https://doi. org/10.1016/j.fgb.2015.08.010

10 Guethlein, LA, Norman, PJ, Hilton, HG, and Parham, P, Co-evolution of MHC class I and variable NK cell receptors in placental mammals. Immunol Rev 267 (2015) 259-82. doi: 10.1111/imr.12326

11 de Groot, NG, Blokhuis, JH, Otting, N, Doxiadis, GGM, and Bontrop, RE, Co-evolution of the MHC class I and KIR gene families in rhesus macaques: ancestry and plasticity. Immunological Reviews 267 (2015) 228-245. doi: https://doi.org/10.1111/imr.12313

12 Eid, J, Fehr, A, Gray, J, Luong, K, Lyle, J, Otto, G, et al., Real-Time DNA Sequencing from Single Polymerase Molecules. Science (New York, N.Y.) 323 (2009) 133. doi: 10.1126/science.1162986

13 Jain, M, Fiddes, IT, Miga, KH, Olsen, HE, Paten, B, and Akeson, M, Improved data analysis for the MinION nanopore sequencer. Nature Methods 12 (2015) 351-356. doi: 10.1038/nmeth.3290

14 Cai, R, Dong, Y, Fang, M, Guo, C, and Ma, X, De novo genome assembly of a Han Chinese male and genomewide detection of structural variants using Oxford Nanopore sequencing. Molecular Genetics and Genomics 295 (2020) 871-876. doi: 10.1007/s00438-020-01672-y

15 Jain, M, Koren, S, Miga, KH, Quick, J, Rand, AC, Sasani, TA, et al., Nanopore sequencing and assembly of a human genome with ultra-long reads. Nature Biotechnology 36 (2018) 338-345. doi: 10.1038/nbt.4060

16 Shafin, K, Pesout, T, Lorig-Roach, R, Haukness, M, Olsen, HE, Bosworth, C, et al., Nanopore sequencing and the Shasta toolkit enable efficient de novo assembly of eleven human genomes. Nature Biotechnology (2020). doi: 10.1038/s41587-020-0503-6

17 Fuselli, S, Baptista, RP, Panziera, A, Magi, A, Guglielmi, S, Tonin, R, et al., A new hybrid approach for MHC genotyping: high-throughput NGS and long read MinION nanopore sequencing, with application to the nonmodel vertebrate Alpine chamois (Rupicapra rupicapra). Heredity 121 (2018) 293-303. doi: 10.1038/s41437018-0070-5

18 Gabrieli, T, Sharim, H, Fridman, D, Arbib, N, Michaeli, Y, and Ebenstein, Y, Selective nanopore sequencing of human BRCA1 by Cas9-assisted targeting of chromosome segments (CATCH). Nucleic acids research 46 (2018) e87. doi: 10.1093/nar/gky411 
19 Giesselmann, P, Brändl, B, Raimondeau, E, Bowen, R, Rohrandt, C, Tandon, R, et al., Analysis of short tandem repeat expansions and their methylation state with nanopore sequencing. Nature Biotechnology 37 (2019) 1478-1481. doi: 10.1038/s41587-019-0293-x

20 Gilpatrick, T, Lee, I, Graham, JE, Raimondeau, E, Bowen, R, Heron, A, et al., Targeted nanopore sequencing with Cas9-guided adapter ligation. Nature Biotechnology 38 (2020) 433-438. doi: 10.1038/s41587-020-0407-5

21 Stangl, C, de Blank, S, Renkens, I, Westera, L, Verbeek, T, Valle-Inclan, JE, et al., Partner independent fusion gene detection by multiplexed CRISPR-Cas9 enrichment and long read nanopore sequencing. Nature Communications 11 (2020) 2861. doi: 10.1038/s41467-020-16641-7

22 Watson, CM, Crinnion, LA, Hewitt, S, Bates, J, Robinson, R, Carr, IM, et al., Cas9-based enrichment and singlemolecule sequencing for precise characterization of genomic duplications. Laboratory Investigation 100 (2020) 135-146. doi: 10.1038/s41374-019-0283-0

23 Bashirova, AA, Thomas, R, and Carrington, M, HLA/KIR restraint of HIV: surviving the fittest. Annu Rev Immunol 29 (2011) 295-317. doi: 10.1146/annurev-immunol-031210-101332

24 Farag, SS, Bacigalupo, A, Eapen, M, Hurley, C, Dupont, B, Caligiuri, MA, et al., The Effect of KIR Ligand Incompatibility on the Outcome of Unrelated Donor Transplantation: A Report from the Center for International Blood and Marrow Transplant Research, the European Blood and Marrow Transplant Registry, and the Dutch Registry. Biology of Blood and Marrow Transplantation 12 (2006) 876-884. doi: https://doi.org/10.1016/j. bbmt.2006.05.007

25 Yu, P, Qi, F, Xu, Y, Li, F, Liu, P, Liu, J, et al., Age-related rhesus macaque models of COVID-19. Animal Models and Experimental Medicine 3 (2020) 93-97. doi: https://doi.org/10.1002/ame2.12108

26 Hatziioannou, T, Ambrose, Z, Chung, NPY, Piatak, M, Yuan, F, Trubey, CM, et al., A macaque model of HIV-1 infection. Proceedings of the National Academy of Sciences 106 (2009) 4425. doi: 10.1073/pnas.0812587106

27 Estes, JD, Wong, SW, and Brenchley, JM, Nonhuman primate models of human viral infections. Nature Reviews Immunology 18 (2018) 390-404. doi: 10.1038/s41577-018-0005-7

28 Bruijnesteijn, J, de Groot, NG, Otting, N, Maccari, G, Guethlein, LA, Robinson, J, et al., Nomenclature report for killer-cell immunoglobulin-like receptors (KIR) in macaque species: new genes/alleles, renaming recombinant entities and IPD-NHKIR updates. Immunogenetics 72 (2020) 37-47. doi: 10.1007/s00251-019-01135-8

29 Marsh, SG, Parham, P, Dupont, B, Geraghty, DE, Trowsdale, J, Middleton, D, et al., Killer-cell immunoglobulin-like receptor (KIR) nomenclature report, 2002. Immunogenetics 55 (2003) 220-6. doi: 10.1007/s00251-003-0571-z

30 Maccari, G, Robinson, J, Hammond, JA, and Marsh, SGE, The IPD Project: a centralised resource for the study of polymorphism in genes of the immune system. Immunogenetics 72 (2020) 49-55. doi: 10.1007/s00251-01901133-w

31 Guethlein, LA, Older Aguilar, AM, Abi-Rached, L, and Parham, P, Evolution of Killer Cell Ig-Like Receptor (KIR) Genes: Definition of an Orangutan KIR Haplotype Reveals Expansion of Lineage III KIR Associated with the Emergence of MHC-C. The Journal of Immunology 179 (2007) 491. doi: 10.4049/jimmunol.179.1.491

32 de Groot, NG, Blokhuis, JH, Otting, N, Doxiadis, GG, and Bontrop, RE, Co-evolution of the MHC class I and KIR gene families in rhesus macaques: ancestry and plasticity. Immunol Rev 267 (2015) 228-45. doi: 10.1111/ imr.12313

33 Pyo, C-W, Wang, R, Vu, Q, Cereb, N, Yang, SY, Duh, F-M, et al., Recombinant structures expand and contract inter and intragenic diversification at the KIR locus. BMC Genomics 14 (2013) 89. doi: 10.1186/1471-2164-1489

34 Chan, HW, Kurago, ZB, Stewart, CA, Wilson, MJ, Martin, MP, Mace, BE, et al., DNA methylation maintains allele-specific KIR gene expression in human natural killer cells. J Exp Med 197 (2003) 245-55. doi: 10.1084/ jem.20021127

35 Bruijnesteijn, J, de Groot, N, van der Wiel, MKH, Otting, N, de Vos-Rouweler, AJM, de Groot, NG, et al., Unparalleled Rapid Evolution of KIR Genes in Rhesus and Cynomolgus Macaque Populations. The Journal of Immunology (2020) ji1901140. doi: 10.4049/jimmunol.1901140

36 Bruijnesteijn, J, van der Wiel, MKH, Swelsen, WTN, Otting, N, de Vos-Rouweler, AJM, Elferink, D, et al., Human and Rhesus Macaque KIR Haplotypes Defined by Their Transcriptomes. Journal of immunology (Baltimore, Md. : 1950) 200 (2018) 1692-1701. doi: 10.4049/jimmunol.1701480 
37 Bimber, BN, Moreland, AJ, Wiseman, RW, Hughes, AL, and O'Connor, DH, Complete characterization of killer Ig-like receptor (KIR) haplotypes in Mauritian cynomolgus macaques: novel insights into nonhuman primate KIR gene content and organization. Journal of immunology (Baltimore, Md. : 1950) 181 (2008) 6301-8. doi: 10.4049/jimmunol.181.9.6301

38 Dutcher, S, Fulton,R. and Lindsay,T., Macaca mulatta isolate AG07107 chromosome 19, whole genome shotgun sequence, 2018.

39 Sambrook, JG, Bashirova, A, Palmer, S, Sims, S, Trowsdale, J, Abi-Rached, L, et al., Single haplotype analysis demonstrates rapid evolution of the killer immunoglobulin-like receptor (KIR) loci in primates. Genome research 15 (2005) 25-35. doi: 10.1101/gr.2381205

40 Warren, WC, Harris, RA, Haukness, M, Fiddes, IT, Murali, SC, Fernandes, J, et al., Sequence diversity analyses of an improved rhesus macaque genome enhance its biomedical utility. Science 370 (2020) eabc6617. doi: 10.1126/science.abc6617

41 Benchling, [Biology Software]. Retrieved from https://benchling.com. (2020).

42 Doench, JG, Fusi, N, Sullender, M, Hegde, M, Vaimberg, EW, Donovan, KF, et al., Optimized sgRNA design to maximize activity and minimize off-target effects of CRISPR-Cas9. Nature Biotechnology 34 (2016) 184-191. doi: $10.1038 /$ nbt.3437

43 Hsu, PD, Scott, DA, Weinstein, JA, Ran, FA, Konermann, S, Agarwala, V, et al., DNA targeting specificity of RNAguided Cas9 nucleases. Nature Biotechnology 31 (2013) 827-832. doi: 10.1038/nbt.2647

44 impson, JT, Workman, RE, Zuzarte, PC, David, M, Dursi, LJ, and Timp, W, Detecting DNA cytosine methylation using nanopore sequencing. Nature Methods 14 (2017) 407-410. doi: 10.1038/nmeth.4184

45 De Coster, W, Stovner, EB, and Strazisar, M, Methplotlib: analysis of modified nucleotides from nanopore sequencing. Bioinformatics 36 (2020) 3236-3238. doi: 10.1093/bioinformatics/btaa093

46 Uhrberg, M, Valiante, NM, Shum, BP, Shilling, HG, Lienert-Weidenbach, K, Corliss, B, et al., Human diversity in killer cell inhibitory receptor genes. Immunity 7 (1997) 753-63. doi: 10.1016/s1074-7613(00)80394-5

47 Roe, D, Vierra-Green, C, Pyo, CW, Eng, K, Hall, R, Kuang, R, et al., Revealing complete complex KIR haplotypes phased by long-read sequencing technology. Genes \& Immunity 18 (2017) 127-134. doi: 10.1038/gene.2017.10

48 Nei, M, and Rooney, AP, Concerted and birth-and-death evolution of multigene families. Annu Rev Genet 39 (2005) 121-52. doi: 10.1146/annurev.genet.39.073003.112240

$49 \mathrm{Li}, \mathrm{H}$, Pascal, V, Martin, MP, Carrington, M, and Anderson, SK, Genetic control of variegated KIR gene expression: polymorphisms of the bi-directional KIR3DL1 promoter are associated with distinct frequencies of gene expression. PLoS Genet 4 (2008) e1000254. doi: 10.1371/journal.pgen.1000254

50 Björkström, NK, Béziat, V, Cichocki, F, Liu, LL, Levine, J, Larsson, S, et al., CD8 T cells express randomly selected KIRs with distinct specificities compared with NK cells. Blood 120 (2012) 3455-65. doi: 10.1182/ blood-2012-03-416867

51 Wu, CY, Zhang, B, Kim, H, Anderson, SK, Miller, JS, and Cichocki, F, Ascorbic Acid Promotes KIR Demethylation during Early NK Cell Differentiation. J Immuno/ 205 (2020) 1513-1523. doi: 10.4049/jimmunol.2000212

52 Hiby, SE, Walker, JJ, O'Shaughnessy, KM, Redman, CWG, Carrington, M, Trowsdale, J, et al., Combinations of Maternal KIR and Fetal HLA-C Genes Influence the Risk of Preeclampsia and Reproductive Success. Journal of Experimental Medicine 200 (2004) 957-965. doi: 10.1084/jem.20041214 


\section{Supplementary materials}

Supplementary Table I. Overview of crRNAs to enrich for the human KIR gene region. Benchling software was used to predict potential crRNA sequences for the KIR gene region [43]. The different crRNAs are combined into four different pools. All target sequences were followed by an NGG PAM. The on-target score is determined by the optimized metric of Doench, et al. [44].

\begin{tabular}{|c|c|c|c|c|c|c|}
\hline$\#$ & Pool & Target gene(s) & Cut site & Direction & crRNA sequence & $\begin{array}{l}\text { On- } \\
\text { target } \\
\text { score }\end{array}$ \\
\hline \multicolumn{7}{|c|}{ Gene to gene fragments: Generic crRNAs } \\
\hline 1 & 1 & $\begin{array}{l}\text { KIR2DL/S, KIR3DL/S, KIR2DP1, } \\
\text { KIR3DP1 }\end{array}$ & Intron 3 & Reverse & CATAGAAAGATGGAGATGTG & 71.1 \\
\hline 2 & 2 & $\begin{array}{l}\text { KIR2DL/S, KIR3DL/S, KIR2DP1, } \\
\text { KIR3DP1 }\end{array}$ & Intron 3 & Forward & TGTGAGCAAAGTCAGCATGG & 73.9 \\
\hline 3 & 2 & $\begin{array}{l}\text { KIR2DL/S, KIR3DL/S, KIR2DL4, } \\
\text { KIR3DP1 }\end{array}$ & Intron 4 & Reverse & GACAAGGAAGAACCTCCCTG & 76.0 \\
\hline 4 & 1,2 & $\begin{array}{l}\text { KIR2DL/S, KIR3DL/S, KIR2DL4, } \\
\text { KIR2DP1, KIR3DP1 }\end{array}$ & Intron 2 & Forward & TGTGATTACACTGAGCCCAG & 74.7 \\
\hline 5 & 1,2 & $\begin{array}{l}\text { KIR2DL/S, KIR3DL/S, KIR2DP1, } \\
\text { KIR3DP1 }\end{array}$ & Intron 2 & Forward & СCTATGTGGATGGAGCCTGG & 71.7 \\
\hline 6 & 1,2 & $\begin{array}{l}\text { KIR2DL/S, KIR3DL/S, KIR2DL4, } \\
\text { KIR2DP1 }\end{array}$ & Exon 4 & Reverse & CATGTACCATCTATCCAGGG & 71.8 \\
\hline 7 & 1,2 & $\begin{array}{l}\text { KIR2DL/S, KIR3DL/S, KIR2DP1, } \\
\text { KIR3DP1 }\end{array}$ & Exon 3 & Reverse & GCATCTGTAGGTCCCTGCAA & 70.1 \\
\hline 8 & 1,2 & $K I R 2 D L / S, K I R 2 D P 1$ & Intron 3 & Reverse & CCTGTGATGACGATGTCCAG & 73.6 \\
\hline 9 & 1,2 & $K I R 2 D L / S, K I R 2 D P 1$ & Intron 4 & Forward & AGCGGTCAGGAGAGACCCAG & 65.8 \\
\hline 10 & 1,2 & KIR2DL/S, KIR2DP1 & Intron 4 & Forward & САAACTCATGACCTCAACTG & 72.8 \\
\hline 11 & 1,2 & $K I R 2 D L / S, K I R 2 D P 1$ & Intron 4 & Forward & ACCTTGAAGTCTCAAGACAG & 66.4 \\
\hline 12 & 3 & $\begin{array}{l}\text { KIR2DL/S, KIR3DL/S, KIR2DL4, } \\
\text { KIR2DP1, KIR3DP1 }\end{array}$ & Exon/Intron 4 & Forward & СCTGTGACAGAAACAAGCAG & 75.4 \\
\hline 13 & 3 & KIR3DL/S & Intron 5 & Reverse & GGTGAATGGATAGAGAACTG & 79.2 \\
\hline 14 & 3 & $K I R 3 D L / S$ & Intron 5 & Forward & TTCCTACCAACAGGGTACCA & 61.0 \\
\hline 15 & 3 & KIR3DL/S & Intorn 5 & Forward & GGAGATACAGATAGATCATG & 71.4 \\
\hline \multicolumn{7}{|c|}{ Gene to gene fragments: Specific crRNAs } \\
\hline 16 & 1 & KIR3DL3 & Exon 4 & Reverse & TGACCTGGGAACCCGCATCG & 71.4 \\
\hline 17 & 1 & KIR3DL3 & Intron 3 & Reverse & СCTATGTGGATCGAGCCTGG & 72.9 \\
\hline 18 & 1 & LILRP2 & Exon 2 & Forward & GCATTACACGAACTTCCCCT & 69.8 \\
\hline 19 & 1 & LILRP2 & Intron 3 & Forward & GGAGGTGTCAGCTCAGAACG & 73.6 \\
\hline 20 & 1 & LILRP2 & Exon 3 & Forward & САAATTCACTCTGTACAAGG & 71.5 \\
\hline 21 & 1 & FCAR & Intron 2 & Reverse & CCGACAGTATTAGATCATTG & 67.1 \\
\hline 22 & 1 & FCAR & Intron 2 & Reverse & GAGGAACTAAGAGAACCAAG & 69.3 \\
\hline 23 & 1,2 & KIR2DL4 & Intron 6 & Forward & TAGGCACAACTCCCACACTG & 76.0 \\
\hline 24 & 1,2 & KIR2DL4 & Intron 3 & Forward & TGGGCTCAGTGTAATCACAA & 74.3 \\
\hline 25 & 1,2 & KIR3DP1 & Exon 5 & Reverse & ССTGCCTTAACCATGGGGCG & 58.3 \\
\hline 26 & 1,2 & KIR3DP1 & Exon 5 & Reverse & СTAAGGTCTGACCACTCGTA & 59.8 \\
\hline 27 & 1,3 & $K I R 2 D L 4$ & Intron 6 & Reverse & TCTACACTCAGCTTCCCGAG & 74.8 \\
\hline 28 & 1,3 & KIR3DL2 & Intron 6 & Forward & GATAAGAGGCATGAGCCACG & 72.8 \\
\hline 29 & 1,3 & FCAR & Intron 6 & Reverse & АСTGTGCTCAAATTACATCA & 72.2 \\
\hline 30 & 1,3 & FCAR & Intron 2 & Reverse & GACAACCACACAATAATAGG & 69.2 \\
\hline
\end{tabular}


Supplementary Table I. Continued.

\begin{tabular}{|c|c|c|c|c|c|c|}
\hline \# & Pool & Target gene(s) & Cut site & Direction & crRNA sequence & $\begin{array}{l}\text { On- } \\
\text { target } \\
\text { score }\end{array}$ \\
\hline \multicolumn{7}{|c|}{ Gene fragments: Generic crRNAs } \\
\hline 31 & 4 & $\begin{array}{l}\text { KIR2DL/S, KIR3DL/S, KIR2DP1, } \\
\text { KIR3DP1 }\end{array}$ & Exon/Intron 1 & Forward & TGTGTTGGTGAGTCCTGGAA & 67.7 \\
\hline 32 & 4 & KIR2DS, KIR3DL/S & Intron 1 & Forward & TGAGTCCTGGAAGGGAATCG & 66.3 \\
\hline 33 & 4 & $\begin{array}{l}\text { KIR2DL/S, KIR3DL/S, KIR2DP1, } \\
\text { KIR3DP1 }\end{array}$ & Intron 1 & Forward & GTGGAGATATAGGCCTGGAG & 63.3 \\
\hline 34 & 4 & $\begin{array}{l}\text { KIR2DL/S, KIR3DL/S, KIR2DP1, } \\
\text { KIR3DP1 }\end{array}$ & Exon 9 & Reverse & TCAAGTGAAATGGAGAATTG & 60.1 \\
\hline 35 & 4 & KIR2DL/S, KIR3DL/S, KIR2DP1 & 3' UTR & Reverse & AGGTGGAACAGCATGAGGGA & 63.2 \\
\hline 36 & 4 & $\begin{array}{l}\text { KIR2DL/S, KIR3DL/S, KIR2DP1, } \\
\text { KIR3DP1 }\end{array}$ & Exon 9 & Reverse & TTTCTCTCCAGCAGGCAGTG & 66.4 \\
\hline 37 & 4 & KIR3DL3, KIR2DL4 & Exon 9 & Reverse & СTCCCTAGAAGATCCCATCA & 64.7 \\
\hline 38 & 4 & KIR2DL, KIR2DP1 & Intron 1 & Forward & CCAAGACTCACAGCCCAGTG & 67.0 \\
\hline 39 & 4 & $K I R 2 D L, K I R 2 D P 1$ & Intron 1 & Forward & TTGGGTGCAGGTAGGCACTG & 63.9 \\
\hline 40 & 4 & KIR2DS, KIR2DP1 & Intron 2 & Forward & ACTAGGAAGAGGGGACCCTG & 70.7 \\
\hline 41 & 4 & $K I R 2 D L / S$ & Intron 1 & Forward & CAAGACTCACAGCCCAGTGG & 63.4 \\
\hline 42 & 4 & KIR3DL & Intron 2 & Forward & GTCAAGCTCTGTGAAGACTG & 72.8 \\
\hline 43 & 4 & KIR3DL, KIR2DL4 & Intron 2 & Forward & AGCAGGTCCTCTGAGGACAA & 67.9 \\
\hline 44 & 4 & KIR3DL3, KIR2DL4 & Intron 2 & Forward & ACCCTCCAGCGTTTCCATGA & 62.4 \\
\hline 45 & 4 & KIR2DL & Exon 9 & Reverse & TACACGATGATATCTGTTGG & 72.7 \\
\hline 46 & 4 & KIR2DL/S, KIR3DL3, KIR2DP1 & Exon 9 & Reverse & GTACATGGGAGCTGGCAACC & 63.7 \\
\hline 47 & 4 & $K I R 2 D L / S, K I R 3 D L / S$ & Intron 8 & Reverse & GGGAAATGCTGAGTGAGGGA & 64.6 \\
\hline 48 & 4 & KIR3DL, KIR2DL4 & Intron 7/Exon 8 & Reverse & ACAGCAGCATCTGTAGGAGA & 63.7 \\
\hline 49 & 4 & $\begin{array}{l}\text { KIR2DL, KIR3DL/S, KIR2DL4, } \\
\text { KIR2DP1 }\end{array}$ & Intron 8/Exon 9 & Reverse & TTCATCAGAGTCCTGGAGAG & 62.3 \\
\hline 50 & 4 & KIR3DP1 & Intron 8 & Reverse & CATTGACCTTGGGCACTGCA & 62.9 \\
\hline \multicolumn{7}{|c|}{ Gene fragments: Specific crRNAs } \\
\hline 51 & 4 & KIR2DL4 & 5' UTR & Forward & AATTCAATCGTCCCGTGCAG & 62 \\
\hline 52 & 4 & KIR2DL4 & $5^{\prime}$ UTR & Forward & TGTGTGTTGAGAAGATCATG & 73.2 \\
\hline 53 & 4 & $K I R 2 D L 4$ & 3' UTR & Reverse & GGAAAGGTGGAACAGCACGA & 78.7 \\
\hline 54 & 4 & KIR3DL2 & Intron 1 & Forward & СTTGGCAGCAGGTAGCAGGG & 73.3 \\
\hline
\end{tabular}


Supplementary Table II. Overview of crRNAs to enrich for the rhesus macaque KIR gene region. Benchling software was used to predict potential crRNA sequences for the KIR gene region [43]. The different crRNAs are combined into six different pools. All target sequences were followed by an NGG PAM. The on-target score is determined by the optimized metric of Doench, et al. [44].

\begin{tabular}{|c|c|c|c|c|c|c|}
\hline$\#$ & Pool & Target gene(s) & Cut site & Direction & crRNA sequence & $\begin{array}{l}\text { On- } \\
\text { target } \\
\text { score }\end{array}$ \\
\hline \multicolumn{7}{|c|}{ Gene to gene fragments: Generic crRNAs } \\
\hline 1 & 1 & $\begin{array}{l}\text { KIR2DLO4, KIR2DP, KIR3DL2O, } \\
\text { KIR3DL/S }\end{array}$ & Intron 4 & Forward & GACAAGGAAGAACCTCCCTG & 76 \\
\hline 2 & 1 & $\begin{array}{l}\text { KIR1D, KIR2DLO4, KIR1D, } \\
\text { KIR3DL/S }\end{array}$ & Exon 5 & Forward & CATGTACCATCTATCCAGGG & 72 \\
\hline 3 & 1 & KIR1D, KIR2DP, KIR3DL/S & Intron 4 & Forward & CAGACCAGGTGTCATAACAG & 72 \\
\hline 4 & 1 & KIR2DL04, KIR3DL2O, KIR3DL/S & Intron 3 & Reverse & GAAAATTATCACAAACATGG & 70 \\
\hline 5 & 1 & KIR1D, KIR2DP, KIR3DL/S & Intron 4 & Reverse & CGGCATCTGTAGGTGCCACA & 66.5 \\
\hline 6 & 2 & KIR3DL20, KIR3DL/S & Intron 3 & Forward & AGATGGAGGGACCTGCACCA & 76 \\
\hline 7 & 2 & KIR1D, KIR2DP, KIR3DL/S & Intron 5 & Forward & ATAACAGAGGACAGACACAG & 81.2 \\
\hline 8 & 2 & KIR1D, KIR2DP, KIR3DL/S & Intron 5 & Forward & CAGACCAGGTGTCATAACAG & 77 \\
\hline 9 & 1,2 & KIR2DLO4, KIR3DL/S & Intron 3 & Reverse & TCCACCTTCAAAGCCCACAG & 72.6 \\
\hline \multicolumn{7}{|c|}{ Gene to gene fragments: Specific crRNAs } \\
\hline 10 & 1 & KIR3DL20 & Exon 4 & Reverse & ССTATGATCACGATGTCCAG & 71 \\
\hline 11 & 2 & KIR2DLO4 & Intron 5 & Reverse & СCTAAAAAGAGAACTTCCAG & 71 \\
\hline 12 & 3 & LILRA6 & Intron 6 & Forward & GGGAGGACTGAAATGAGATA & 61.5 \\
\hline 13 & 3 & KIR2DLO4 & Intron 5 & Reverse & AATTAGTACAACTTTCACAG & 74 \\
\hline 14 & 4 & KIR2DLO4 & Intron 1 & Forward & AGATTCGGGGTCTCCCAACA & 70.4 \\
\hline 15 & 4 & KIR2DLO4 & Intron 1 & Forward & AGTGAACGTTCAGCCCAGCG & 70 \\
\hline 16 & 4 & FcaR & Intron 2 & Reverse & ССTTTAAATGAAGTGACAGG & 66.6 \\
\hline 17 & $1,2,4$ & FCaR & Intron 2 & Reverse & TGGGCAGTCTAACACAGGCA & 67.5 \\
\hline 18 & $1,2,4$ & FcaR & Intron 2 & Reverse & СTTCATGGGCAGTCTAACAC & 62 \\
\hline 19 & $1,2,4$ & KIR1D & Exon 3 & Reverse & СTGTGACCATGATCGCCAGG & 78.3 \\
\hline 20 & 1,3 & LILRA6 & Intron 6 & Forward & AGACTGAGGATAGAAGATGG & 72 \\
\hline 21 & 1,3 & LILRAG & Intron 6 & Forward & AGGACTGAAATGAGATACGG & 72 \\
\hline
\end{tabular}


Supplementary Table II. Continued.

\begin{tabular}{|c|c|c|c|c|c|c|}
\hline$\#$ & Pool & Target gene(s) & Cut site & Direction & crRNA sequence & $\begin{array}{l}\text { On- } \\
\text { target } \\
\text { score }\end{array}$ \\
\hline \multicolumn{7}{|c|}{ Gene fragments: Generic crRNAs } \\
\hline 22 & 5 & KIR3DL20, KIR1D, KIR3DL/S & 3' UTR & Reverse & GGACTAGGAAGTAAGCCAGT & 63 \\
\hline 23 & 5 & KIR3DL20, KIR1D, KIR3DL & 3' UTR & Reverse & ACGTTCAGATTCGTGGTGTG & 63 \\
\hline 24 & 5 & KIR3DL20, KIR2DLO4, KIR3DL/S & 3' UTR & Reverse & CTGAAAAATCGTCTGAGGGG & 64 \\
\hline 25 & 5 & $\begin{array}{l}\text { KIR3DL20, KIR1D, KIR2DLO4, } \\
\text { KIR3DL/S }\end{array}$ & 3' UTR & Reverse & AGGTGGAACAGCATGAGGGA & 63.7 \\
\hline 26 & 5 & KIR3DL2O, KIR2DL04, KIR3DS & 3' UTR & Reverse & AAGACTGAAAAATCGTCTGA & 63.3 \\
\hline 27 & 5 & KIR1D, KIR3DL/S & 5' UTR & Forward & GGTCAGCACGTCAACTGCAT & 61.4 \\
\hline 28 & 5 & KIR3DL/S & 5' UTR & Forward & TGGTCATAGTGAAGGACACA & 66 \\
\hline 29 & 5 & KIR3DL & 3' UTR & Reverse & ATGTTCAGATTCGTGGTGTG & 63 \\
\hline 30 & 6 & KIR3DL20, KIR1D & 3' UTR & Forward & CATCCAAAAATGCAGCACAT & 67 \\
\hline 31 & 6 & KIR3DL20, KIR3DL/S & Intron 7 & Reverse & AAGTCATCAAAACAGCTGGG & 76.4 \\
\hline 32 & 6 & $\begin{array}{l}\text { KIR3DL2O, KIR1D, KIR2DLO4, } \\
\text { KIR3DL/S }\end{array}$ & Exon 9 & Reverse & GTGCGTATGTCACCTCCTGA & 61.8 \\
\hline 33 & 6 & KIR1D, KIR3DL/S & Exon 2 & Forward & CAGAGGGCCTGTCCACACAC & 68.7 \\
\hline 34 & 6 & KIR1D, KIR2DLO4, KIR3DL/S & Intron 2 & Forward & TAGGAACAGCAGGTCCTCTG & 67.3 \\
\hline 35 & 6 & KIR3DL & Exon 9 & Reverse & TGGAAGTTCCGTGTACACGC & 61.6 \\
\hline 36 & 6 & KIR3DL & Exon 9 & Reverse & TTTTTCCCTGTGTGAGAACG & 66.7 \\
\hline \multicolumn{7}{|c|}{ Gene fragments: Specific crRNAs } \\
\hline 37 & 5 & KIR2DLO4 & 5' UTR/Exon 1 & Forward & CACCAAGTCCATGTCGCCCA & 61.1 \\
\hline 38 & 5 & KIR2DLO4 & 5' UTR & Forward & GCAGTGTTCCGGTATCAAGT & 62.8 \\
\hline 39 & 5 & KIR3DL20 & 5' UTR & Forward & TGGCAATAGTGAAGGATGCA & 62.4 \\
\hline 40 & 5 & KIR3DL20 & 5' UTR & Forward & TACGTCATCCTCTCATGATG & 61.2 \\
\hline 41 & 6 & KIR2DLO4 & 5' UTR & Reverse & TACGAACAAACTGAATCCCG & 70 \\
\hline 42 & 6 & KIR2DLO4 & 5' UTR & Reverse & ATGATCTTCTCAATACATGT & 62.5 \\
\hline 43 & 6 & KIR3DL20 & Intron 2 & Forward & ACATGAGGCAGGCAGCACAA & 70.3 \\
\hline 44 & 6 & KIR3DL20 & Intron 2 & Forward & GATGATTTTCTTGAAACATG & 63.3 \\
\hline 45 & 6 & KIR3DL20 & 3' UTR & Forward & TTGAATTGACACAAATGGAG & 64.8 \\
\hline
\end{tabular}






\section{The genetic mechanisms driving diversification of the $K I R$ gene cluster in primates}

Jesse Bruijnesteijn ${ }^{1}$, Natasja G. de Groot ${ }^{1}$ and Ronald E. Bontrop ${ }^{1,2}$

${ }^{1}$ Comparative Genetics and Refinement, Biomedical Primate Research Centre, Rijswijk, The Netherlands

${ }^{2}$ Theoretical Biology and Bioinformatics, Utrecht University, Utrecht, The Netherlands 


\begin{abstract}
The activity and function of natural killer (NK) cells are modulated through the interactions of multiple receptor families, of which some recognize $\mathrm{MHC}$ class I molecules. The high level of MHC class I polymorphism requires their ligands either to interact with conserved epitopes, as is utilized by the NKG2A receptor family, or to coevolve with the MHC class I allelic variation, which task is taken up by the killer cell immunoglobulin-like receptor (KIR) family. Multiple molecular mechanisms are responsible for the diversification of the $K I R$ gene system, and include abundant chromosomal recombination, high mutation rates, alternative splicing, and variegated expression. The combination of these genetic mechanisms generates a compound array of diversity as is reflected by the contraction and expansion of KIR haplotypes, frequent birth of fusion genes, allelic polymorphism, structurally distinct isoforms, and variegated expression, which is in contrast to the mainly allelic nature of MHC class I polymorphism in humans. A comparison of the thoroughly studied human and macaque KIR gene repertoires demonstrates a similar evolutionarily conserved toolbox, through which selective forces drove and maintained the diversified nature of the KIR gene cluster. This hypothesis is further supported by the comparative genetics of KIR haplotypes and genes in other primate species. The complex nature of the KIR gene system has an impact upon the education, activity, and function of NK cells in coherence with an individual's MHC class I repertoire and pathogenic encounters. Although selection operates on an individual, the continuous diversification of the KIR gene system in primates might protect populations against evolving pathogens.
\end{abstract}

\title{
Introduction
}

The innate and adaptive arms of the immune system are interconnected, and feature several effector functions that provide efficient and specific protection against infection and tumor formation. Major components of the adaptive arm comprise $T$ and B lymphocytes characterized by rearranging antigen receptors, which exert cytotoxic and humoral immunity, respectively. The cytotoxicity mediated by $T$ lymphocytes highly depends on the presentation of intracellular antigen segments derived from pathogens by MHC class I molecules and subsequent clonal expansion of cells with specific receptors. A third type of lymphocytes bridge the innate and adaptive immune response, and comprises natural killer (NK) cells, which participate, for instance, in the recognition and elimination of aberrant cells that downregulate their MHC class I expression to evade detection by T lymphocytes [1]. Without prior priming or clonal expansion, inhibitory and activating receptors on the NK cell surface interact with MHC class I molecules on nucleated cells to modulate NK cell effector functions, which include the killing of target cells by the release of cytolytic proteins and the regulation of other immune cells by the secretion of cytokines [2]. The genes encoding the MHC class I molecules are considered the most polymorphic genes known in vertebrates, a 
phenomenon that resulted from selective pressure to adapt to the rapid diversification of pathogens. This extended repertoire of MHC class I genes and alleles requires the NK cell receptors to co-evolve to maintain a functional relation with their ligands. The recognition of MHC class I molecules by NK cells involves two receptor families: the conserved CD94:NKG2A receptors and the highly polymorphic and diverse killer cell immunoglobulin-like receptors (KIR). Both receptor families consist of inhibitory and activating members. Their engagement with MHC class I molecules calibrates the responsiveness of NK cells through a continuous educational process, which largely controls subsequent NK cell activity $[3,4]$. The KIR receptors are encoded within the Leukocyte Receptor Complex (LRC) on chromosome 19q13.4, and share this genomic region with other structurally similar immune-regulators, such as the leukocyte Ig-like receptors (LILRs) and the leukocyte-associated Ig-like receptors (LAIRs) (Figure 1) [5]. Based on different Alu elements that can be regarded as a molecular clock, the initial expansion of the primate $K I R$ gene cluster is estimated to date back to approximately 31 to 44 million years ago. This process continued, and is currently reflected by extensive gene duplications and point mutations [6]. Different diversifying mechanisms

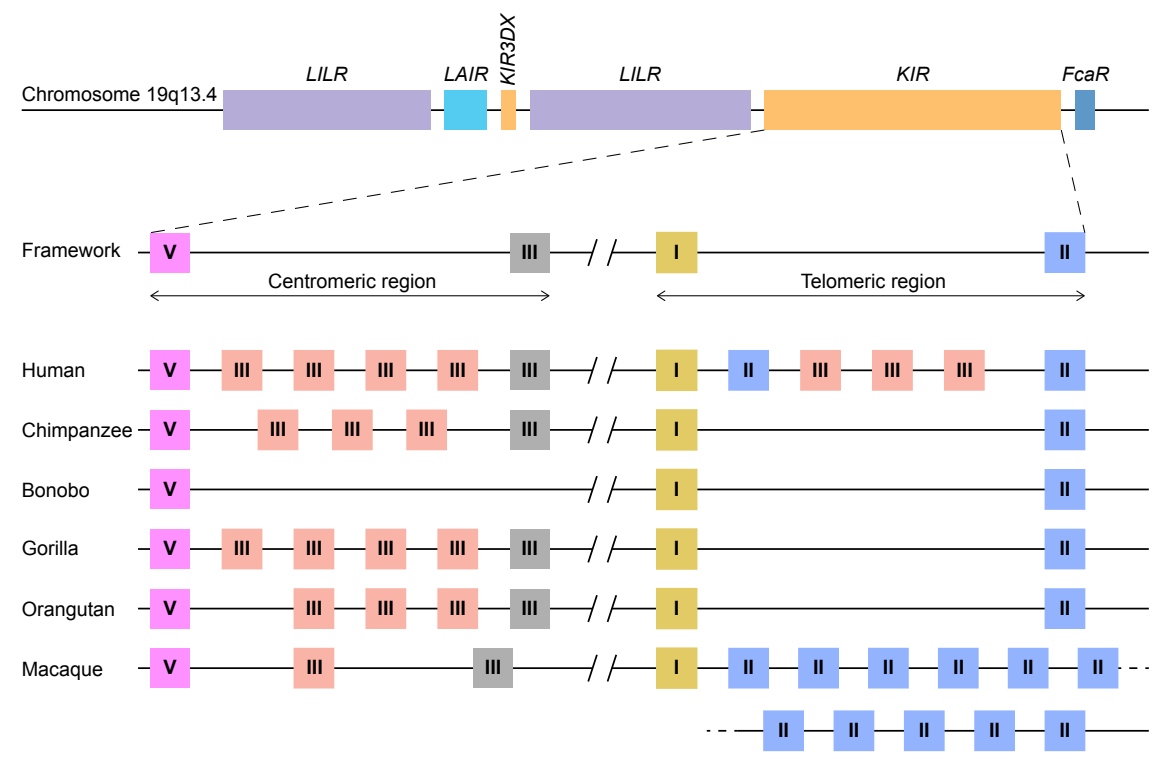

Figure 1. KIR haplotype organizations in different primate species. A schematic overview of the Leukocyte Receptor Complex (LRC) on chromosome 19q13.4 and KIR haplotype organizations in different primate species. A fixed copy of the KIR3DX gene is located within the primate LILR gene cluster, whereas the expanded $K I R$ gene cluster is flanked by the LILR and FcaR genes. The expansion involved four different $K I R$ gene lineages $-\mathrm{I}, \mathrm{II}, \mathrm{II}$, and $\mathrm{V}$ - the members of which are indicated as yellow, blue, red/grey, and pink boxes, respectively. The grey lineage III boxes represent pseudogenes. In most hominoids, KIR haplotype organizations follow a standard framework, in which the centromeric and telomeric regions are bordered by genes from lineages $\mathrm{V}$ and III, and lineages I and II, respectively. A relatively large non-coding segment separates the centromeric and telomeric haplotype sections 
in combination with evolutionary selective factors propel the complex KIR gene content at the individual level but also at the population and species-specific level, which all together contribute to the heterogeneity of NK cell subsets and their activity. The KIR gene diversification is not limited to humans. Comparative analyses that include other primate species might help in gaining a thorough understanding of the evolutionary processes that resulted in the diversification of this gene system. In the following sections, we will discuss the different genetic mechanisms that drove the evolution of the highly plastic KIR gene system in hominoids (humans and great apes) and Old World monkeys, and how this might influence their NK cell response.

\section{Co-evolution of $M H C$ and $K I R$ genes}

The complex KIR gene system requires a comprehensive nomenclature guideline for the different genes and allotypes in order to distinguish the corresponding receptors by their structure and signaling potential [7-9]. Receptors may contain one to three Ig-like domains, which are encoded by exon 3 (D0 domain), exon 4 (D1 domain), and exon 5 (D2 domain), and are referred to as KIR1D, KIR2D, and KIR3D in the official nomenclature. Further classification defines the inhibitory or activating signaling function of the KIR receptors, which is characterized by either a long or short cytoplasmic tail, respectively, and specified with an " $\mathrm{L}$ " or an " $\mathrm{S}$ " following the domain number denotation. The long cytoplasmic tail contains one or two immune tyrosinebased inhibitory motifs (ITIMs), whereas the signal transduction of activating KIR depends on the interaction with an adaptor molecule that includes an immune tyrosine-based activating motif (ITAM) such as DAP12. Pseudogenes are indicated with a "P" (e.g., KIR3DP). In addition, a four-character species designation is included in front of the KIR acronym (e.g., Mamu-KIR3DL2O in rhesus macaques; macaca mulatta).

The mammalian KIR genes originate from two progenitor gene lineages: KIR3DX and KIR3DL. The KIR3DX lineage is represented by a single gene copy located in the center of the LILR gene cluster (Figure 1). The gene is fixed in most primate species, and its function is currently unknown [10]. This lineage is, however, expanded in cattle, and encodes multiple inhibitory and a single activating functional KIR3DX receptor, which interact with an expanded repertoire of classical MHC molecules [11, 12]. In contrast, the KIR3DL lineage expanded in primates and was diversified by duplications, deletions, and recombinations, which resulted in an elaborated KIR gene family. Based on their structure, ligand specificity, and/or phylogenetic analysis, the primate KIR receptors are divided into four lineages. Lineage I genes encode receptors with a D0-D2 domain configuration; lineage II is defined by the specificity for subtypes of HLA-A and -B in humans; lineage III includes receptors with D1-D2 and D0-D1-D2 domain configurations; and lineage $\mathrm{V}$ is represented by human KIR3DL3 and its orthologs. In the primate species studied, at least one KIR gene was discovered for each lineage, which indicates that gene duplication and diversification predates primate speciation. The subsequent lineage expansions are, however, species specific (Table I). 
Table I. The number of KIR genes defined per primate species indicated per lineage.

\begin{tabular}{|c|c|c|c|c|c|c|c|c|c|c|c|c|c|c|c|c|c|}
\hline & & \multirow{2}{*}{\multicolumn{3}{|c|}{ Lineage I }} & & & & & & & & & & & & & \\
\hline & & & & & \multicolumn{3}{|c|}{ Lineage II } & \multicolumn{3}{|c|}{ Lineage III } & \multicolumn{3}{|c|}{ Lineage V } & \multicolumn{4}{|c|}{ Total } \\
\hline & & & $A^{*}$ & $\mathbf{P}$ & 1 & $\mathbf{A}$ & $\mathbf{P}$ & 1 & A & $\mathbf{P}$ & 1 & A & $\mathbf{P}$ & 1 & A & $\mathbf{P}$ & Total \\
\hline Human & Hosa & 2 & 1 & 0 & 2 & 1 & 0 & 3 & 5 & 2 & 1 & 0 & 0 & 8 & 7 & 2 & 17 \\
\hline Chimpanzee & Patr & 1 & 1 & 0 & 1 & 0 & 0 & 6 & 3 & 0 & 1 & 0 & 0 & 9 & 4 & 0 & 13 \\
\hline Bonobo & Papa & 1 & 1 & 0 & 3 & 1 & 0 & 2 & 0 & 0 & 1 & 0 & 0 & 7 & 2 & 0 & 9 \\
\hline Gorilla & Gogo & 1 & 1 & 0 & 1 & 0 & 0 & 5 & 1 & 0 & 1 & 0 & 0 & 8 & 2 & 0 & 10 \\
\hline $\begin{array}{l}\text { Bornean } \\
\text { organgutan }\end{array}$ & Popy & 1 & 1 & 0 & 1 & 1 & 0 & 2 & 3 & 0 & 1 & 0 & 0 & 5 & 5 & 0 & 10 \\
\hline $\begin{array}{l}\text { Sumatran } \\
\text { organgutan }\end{array}$ & Poab & 1 & 1 & 0 & 1 & 1 & 0 & 3 & 3 & 0 & 1 & 0 & 0 & 6 & 5 & 0 & 11 \\
\hline $\begin{array}{l}\text { Rhesus } \\
\text { macaque }\end{array}$ & Mamu & 0 & 1 & 0 & 31 & 23 & 0 & 1 & 0 & 1 & 1 & 0 & 0 & 33 & 24 & 1 & 58 \\
\hline $\begin{array}{l}\text { Cynomolgus } \\
\text { macaque }\end{array}$ & Mafa & 0 & 1 & 0 & 26 & 30 & 0 & 1 & 0 & 1 & 1 & 0 & 0 & 28 & 31 & 1 & 60 \\
\hline
\end{tabular}

*KIR2DL4 is considered an activating KIR gene.

I=Inhibitory, $\mathbf{A}=$ Activating, $\mathbf{P}=$ Pseudogene

Lineage I and $V K I R$ genes have a conserved nature in all primate species examined, and comprise, respectively, KIR2DL4 and KIR2DL5, and KIR3DL3, or a similar structure, such as Mamu-KIR3DL2O in rhesus macaques. More extensive and species-specific expansions are reported for KIR genes that cluster into lineages II and III (Table I), and the data suggest that this coincides with the evolution of their MHC class I ligands. Therefore, diversification of the lineage II and III KIR genes might be indirectly propelled by the adaption of the MHC class I molecules to pathogenic encounters. For hominoids, this section of co-evolution of KIR and $\mathrm{MHC}$ has been comprehensively reviewed by Wroblewski and colleagues [13]. In short, the $M H C$ gene content in great apes displays to a limited extent a variable number of $M H C-A,-B$, and $-C$ genes per haplotype (Table II). MHC-C, which originated from a duplication of an $M H C-B$ gene, is fixed in all hominoids except for orangutans, where it is present on about half of the haplotypes [14]. In addition, the epitopes recognized by the relevant KIR are differentially distributed across the different MHC class I genes (Table II). The C1 and C2 epitopes, for example, are absent in bonobos and orangutans, respectively, whereas the A3/A11 epitope is only defined on HLA-A molecules. The hominoid MHC class I evolution is accompanied by the reduction and refinement of KIR specific for MHC-A and-B, which is reflected in their limited number of lineage II KIR receptors, whereas the emergence and fixation of $\mathrm{MHC}-\mathrm{C}$ in humans, chimpanzees, and gorillas drove the expansion and specialization of lineage III KIR (Table I) [13].

Old World monkeys, including macaques, lack an MHC-C ortholog, but instead display extensive copy number variation regarding polymorphic MHC-A and -B genes, as opposed to the fixed number of MHC class I genes in hominoids (Table II) [15-18]. The expression level of the different MHC-A and -B molecules, however, varies 
Table 2. The expansion of $M H C$ class I genes in different primate species. Indicated are the number of genes present on a single chromosome and the KIR-recognizing epitopes that may be encoded by allotypes. The frequencies of the different epitopes vary per gene and species. In macaques, on average 1- 3 MHC-B genes are highly transcribed (majors), whereas the total number of genes on a single MHC haplotype can reach up to 19 copies, including low transcribed genes (minors) as well as pseudogenes [15-20].

\begin{tabular}{lcccccc}
\cline { 2 - 7 } & \multicolumn{2}{c}{ MHC-A } & \multicolumn{2}{c}{ MHC-B } & \multicolumn{2}{c}{ MHC-C } \\
\cline { 2 - 7 } & \# genes & KIR-epitopes & \# genes & KIR-epitopes & \# genes & KIR-epitopes \\
\hline Human & 1 & A3/A11, Bw4 & 1 & Bw4, C1 & 1 & C1, C2 \\
\hline Chimpanzee & 1 & - & 1 & Bw4, C1 & 1 & C1, C2 \\
\hline Bonobo & 1 & - & 1 & Bw4, C1 & 1 & C2 \\
\hline Gorilla & $1\left(2^{*}\right)$ & Bw4 & $1-2$ & Bw4, C1 & 1 & C1, C2 \\
\hline Orangutan & 1 & - & $2-4$ & Bw4, C1 & $0-1$ & C1 \\
\hline Macaque & $1-3$ & Bw4, Bw6 & $1-3(<19)$ & Bw4, Bw6 & - & - \\
\hline
\end{tabular}

*Gorilla's have an additional MHC-A related gene, named Gogo-Oko

considerably in macaques. It is generally accepted that per haplotype at least a single MHC-A and 1 to 3 MHC-B genes are characterized by high transcription, and are referred to as "majors", whereas the other MHC class I genes have lower transcription levels ("minors"), or may be pseudogenes. The differential transcription suggests a more classical function for the major MHC molecules, such as antigen presentation, whereas the minors might exert more specialized functions $[19,20]$. Only a few interactions of macaque $\mathrm{MHC}$ and $\mathrm{KIR}$ are documented, and, so far, all interactions involved lineage II KIR that recognize Bw4 and Bw6 epitopes on MHC-A and-B allotypes (Table II) [21-25]. This putative lineage II specificity for the copious macaque MHC class I repertoire coincides with an extensive ligand expansion, and, thus far, 54 and 56 different lineage II KIR genes have been documented for rhesus and cynomolgus macaques, respectively (Table I) [7]. Like the majors and minors for the MHC system, the KIR genes may display differential expression levels, which are modulated by sequence polymorphisms and by an individual's MHC class / repertoire [26-28]. Lineage III KIR genes, which encode ligands for $\mathrm{MHC}-\mathrm{C}$ in hominoids and were subject to expansion, are represented in macaques by a single gene and encodes a receptor with only the D1 extracellular domain (KIR1D). Its presence on $22 \%$ and $82 \%$ of the rhesus and cynomolgus macaque KIR haplotypes, respectively, suggests a balancing selection for this structurally modified receptor, which might execute a function other than conventional $\mathrm{MHC}$ recognition [29].

The maximal expression of six distinct MHC class / genes in most hominoids and the specialization of MHC-C as ligand for lineage III KIR is in line with their modest KIR gene expansion (Tables I and II). Macaques may harbor over 20 distinct MHC class I genes in one individual, of which only a few are dominantly expressed and considered to be majors. The expanded $\mathrm{MHC}$ repertoire in macaques probably propelled the extensive expansion and differential expression of their lineage II KIR. The balanced expansion of the $M H C$ and KIR gene systems in primates indicates co-evolution in order to maintain a functional relation. 


\section{Transposable elements facilitate chromosomal recombination}

One of the mechanisms responsible for the extensive KIR gene diversification in macaques, and to a lesser extent in hominoids, involves chromosomal rearrangements that are accompanied by deletions and recombination, which may result in the generation of fusion genes (Figure 2A). This type of gene formation may shuffle the binding and signaling domains of different KIR receptors, thereby functionally altering the response potential of KIR family members. The dense head-to-tail arrangement of the KIR genes is likely to facilitate at least in part the chromosomal instability of this gene cluster. A KIR haplotype spans approximately 150 to $350 \mathrm{~kb}$, depending on the number of genes present. Most KIR genes are separated by only $2.5 \mathrm{~kb}$, as opposed to the wider haplotype configurations of more stable and less expanded gene families, such as the LILR gene cluster [6, 30-33]. In addition, the presence of transposable elements, including Alu and LINE elements, in the intergenic and intragenic KIR sequences is another factor that further promotes genetic instability [6, 34-36]. These repetitive elements are present in all primate $K I R$ genes, although with species-specific variation, and drive recombination and genetic deletions [35, 37-39]. For the few completely sequenced fusion KIR genes in humans, the chromosomal breakpoints indeed map in the intragenic transposable elements. This supports the idea that the abundant presence of transposons in the KIR cluster facilitates chromosome fragility, which is reflected by genetic expansion and contraction, and the formation of fusion genes [34, 40, 41]. A considerable number of human fusion KIR genes were generated by reshuffling that involved segments of pseudogenes [34]. The conservation of two pseudogenes in the human KIR repertoire, KIR2DP1 and KIR3DP1, might be explained by their role in promoting recombination events. The human KIR haplotypes that include an apparent fusion gene are represented by relatively low frequencies [42-45]. Positive selection of fusion entities might, however, increase their frequencies in certain populations [45]. Ancient recombination events and subsequent selection might have contributed substantially to the current human KIR repertoire, but the modest expansion of the human KIR genes nowadays indicates limited recent recombination events. In contrast, an excessive number of recombination events are recorded in rhesus and cynomolgus macaques, with the presence of at least one fusion $K I R$ gene on $42 \%$ and $49 \%$ of their haplotypes, respectively $[29,43]$. The abundant presence of fusion genes indicates that in these species the reshuffling of $K I R$ gene segments is an ongoing process that expands the macaque KIR repertoire. Although information on the non-coding regions in the macaque KIR cluster is limited at present, the chromosome instability and consequential recombinations in concert with selection are likely to have driven the extensive expansion of lineage II KIR genes. This fast mode of evolution is further reflected in the relatively low number of orthologs that are shared between the closely related rhesus and cynomolgus macaques and their populations [29].

In all hominoids and Old World monkeys, the $5^{\prime}$ section of the KIR gene cluster is occupied by KIR3DL3 or similar structures, which are considered framework genes and 

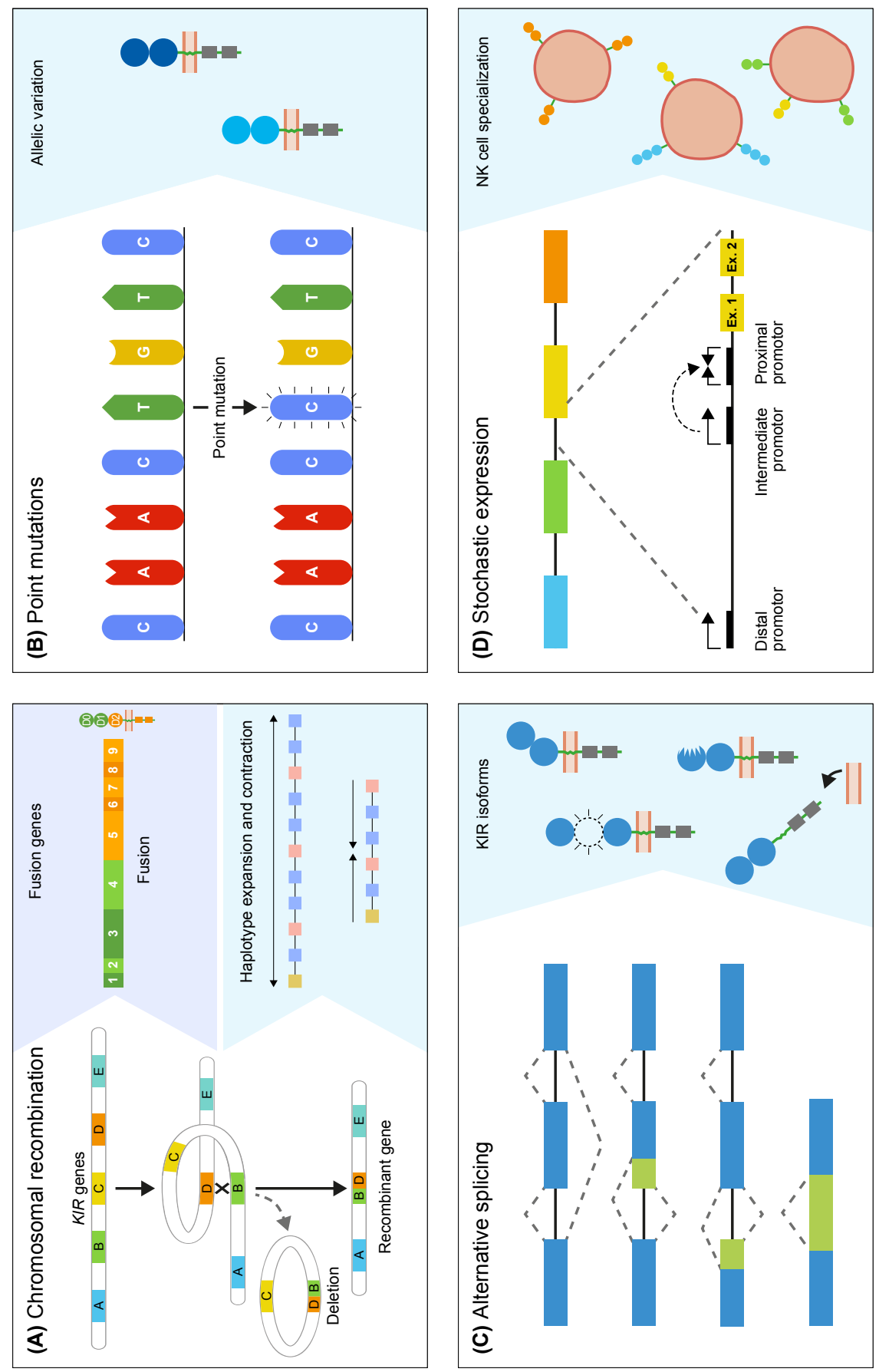
might carry out essential functions. The structure and evolutionary pathway of these lineage $V$ KIR genes is a complex outcome of multiple recombination events [46]. Additional chromosomal rearrangements in rhesus macaques involved the exchange of the cytoplasmic tail of KIR3DL2O with the tails of KIR2DLO4 (lineage I) and KIR1D (lineage III). These recombination events are not conserved in macaque populations, which implies the relatively recent formation of novel gene entities propelled by ancient recombination hotspots [29].

Chromosomal recombination events generate genetic variability in the KIR gene cluster by the formation of fusion genes. Subsequent selection of these novel genes might supply an adaptive and protective strategy in the arms race with rapidly evolving pathogens.

\section{KIR haplotype diversity in primate species}

Chromosomal rearrangements not only generate novel KIR gene entities by recombination but also diversify the haplotype gene content by insertions and deletions of genes (Figure 2A). In general, hominoid KIR haplotypes consist of two genomic regions that are bordered by four framework genes (Figure 1). The proximal half of the haplotype is termed the centromeric region and is defined by KIR3DL3 to $K I R 3 D P 1 / K I R D P$, whereas the distal part, or telomeric region, ranges from KIR2DL4 to KIR3DL2/KIR3DL1. Within these sections, KIR genes of different lineages expanded and contracted during hominoid speciation. In humans, the expansion involved lineage III $K I R$ genes in their centromeric and telomeric regions, whereas expansion in chimpanzees, gorillas, and orangutans expansion took place in the same lineage in the centromeric region only (Figure 1). The human haplotype content ranges from 7 to 12 $K I R$ genes, whereas the number in chimpanzee and orangutan haplotypes stretches from 5 to 11 and 5 to 10 functional KIR genes, respectively. In contrast to other hominoids, bonobos are characterized by a contraction of their KIR region, with only 3-7 KIR genes expressed on a haplotype. The shortest bonobo KIR haplotype consists

Figure 2. The genetic mechanisms propelling diversification. The primate KIR cluster diverged as a result of multiple molecular processes, which together modulate the KIR gene content and expression status. (A) The expansion and contraction of KIR haplotypes is mediated by chromosomal recombinations, which can introduce or remove one or multiple KIR genes. Occasionally, a recombination event is accompanied by the generation of a fusion gene, which functionally and structurally expands the gene repertoire. (B) The KIR genes are further diversified by point mutations in coding and non-coding regions, which generate alleles that encode receptors with different structures, localization, function, and expression. (C) Alternative splicing is another mechanism that has a similar impact on the function and structure of receptors. The blue and green boxes indicate exons and introns. The isoforms are generated by different splice events, which involve alternative splice sites and exon skipping. (D) The differential expression of subsets of KIR receptors on different NK cell clones forms another level of variation that is mediated and maintained by sequence variability in the complex promoter regions and epigenetic modifications. A conjunction of the proximal, intermediate, and distal promoters is required to induce KIR expression. 
of only the framework genes [47]. The contracted bonobo KIR cluster coincides with a reduced nucleotide variation in their $\mathrm{MHC}$ class I repertoire, which might be caused by a bottleneck or pathogen-driven selective sweep after divergence from the chimpanzee's lineage [48-51]. In contrast, a highly variable KIR haplotype content is encountered in the macaque, with 4 to 17 functional KIR genes that mainly map to the telomeric region (Figure 1). The haplotype framework in macaques is less fixed than in hominoids, with only KIR3DL2O expressed on all haplotypes, whereas KIR2DLO4 is present on $70 \%$ of the rhesus macaque haplotypes. A gene orthologous to hominoid KIR3DL2/KIR3DL1 that usually marks the telomeric region is absent.

The diversified KIR haplotypes in hominoids and Old World monkeys stem from a primordial configuration, for which a model has been proposed by Guethlein and colleagues [35]. This model describes abundant duplications and recombination events that eventually formed a conserved haplotype framework in hominoids. The previously mentioned transposable elements are likely propagating these chromosomal rearrangements that continue to mediate the diversification of haplotype configurations. One major hotspot for recombination is mapping in between the centromeric and telomeric regions, which facilitates the reorganization of the different haplotype regions. In addition, KIR haplotypes also display the insertion and deletion of one or multiple KIR genes propelled by unequal crossing-over, which is occasionally accompanied by the formation of a fusion gene [29, 40, 42-44]. In humans, these contractions and expansions, which are mediated by double-stranded breaks at intragenic and intergenic repetitive elements, resulted in haplotypes that expressed 3 to 15 KIR genes [40]. The short haplotypes do not express all framework genes. For instance, the deletion of KIR2DL4 is commonly observed on genotypes defined across different populations $[52,53]$. Approximately $7 \%$ of the human KIR haplotypes are showing indications for contraction and expansion [42]. Although the number of completely defined KIR haplotypes in other hominoids is low, several rare KIR configurations in chimpanzees and orangutans illustrate genetic footprints for insertion and deletion events, which is also occasionally accompanied by the formation of a fusion gene $[54,55]$. In macaques, only two completely sequenced haplotypes are available at present, whereas an abundant number of haplotypes are deduced the transcription level by segregation studies [26, 29, 31, 43, 56, 57]. The presence of multiple highly similar allotypes, encoded by highly similar $K I R$ genes, on a single haplotype indicates an expansion by the insertion of one or more genes. Such events were recorded for $47 \%$ and $26 \%$ of the rhesus and cynomolgus macaque haplotypes, respectively [29]. In contrast, a minimal KIR gene content and the presence of a fusion gene often are indicative of a haplotype contraction. An example of a prominent haplotype reduction in rhesus macaques involved the deletion of the complete centromeric region by an intragenic recombination of KIR3DL2O and KIR2DLO4 [29]. The variable haplotype content and the relatively high number of fusion genes indicate extensive recombination as a mechanism to diversify the macaque KIR gene system in a still ongoing process. This phenomenon is observed to a lesser extent for the KIR haplotypes in hominoids, where the process seems to have relaxed. 


\section{The $K I R$ gene allelic repertoire is expanded by point mutations}

Another level of variation is displayed by allelic polymorphisms, which is explained to a large extent by the occurrence of single nucleotide polymorphisms (SNP) (Figure 2B). These nucleotide variations have a wide-ranging impact, and may modulate the expression level at the cell surface, ligand specificity, interaction strength, and localization of the KIR receptor. Single nucleotide variations in the extracellular D0 and D1 domains of human KIR2DL2*004 and KIR3DL1*004, for example, retain the receptors within the cell, which might be caused by misfolding $[58,59]$. Polymorphisms in KIR2DL3 alleles affect the avidity of the receptor to bind their HLA-C ligands. The low-avidity KIR2DL3*001 and the high-avidity KIR2DL3*005 only differ at three nucleotides in their D1 domain, which alters the orientation of their extracellular domains and thereby their binding strength [60]. Although most KIR disease association studies determine the gene content by the presence and absence of KIR gene sections, and thereby lack allele-level resolution, several studies demonstrated that the functional differences of KIR alleles might also impact health and disease. For example, two KIR2DL1 alleles in the African KhoeSan population evolved by single nucleotide mutations and are associated with a reduced risk for pregnancy disorders [61]. Other associations demonstrated that the highly expressed KIR3DL1 alleles are more protective against disease progression in HIV-infected individuals than lower expressed allotypes, except for the intracellularly retained KIR3DL1*004, which is low in expression but highly protective [62-64].

A total of 1110 human KIR alleles are catalogued in the Immuno Polymorphism Database (IPD-KIR, release 2.9.0), whereas the number of reported alleles for different non-human primate species ranges from $521 \mathrm{KIR}$ alleles in rhesus macaques to $5 \mathrm{KIR}$ alleles in Bornean orangutans (IPD-NHKIR, release 1.2.0.0). These allele numbers may give a distorted view of the actual levels of polymorphism within a species due to the differential number of individuals studied. The high level of allelic polymorphism appears to be at least comparable in humans and macaques. The thoroughly documented allelic polymorphism in humans and macaques reveals a varying number of alleles per KIR gene, with most nucleotide variation exhibited by the framework genes [7, 29, 44]. In addition, a high number of alleles were reported for certain KIR genes located on the telomeric haplotype region in humans (KIR3DL1, KIR2DS4) and the highly frequent inhibitory KIR genes in macaques (KIR3DLO1, KIR3DLO7). An expansion of the allele numbers for the frequently expressed $K I R$ genes might indicate a continuous role in co-evolution with particular pathogens. The less common KIR genes, which include mostly activating KIR, vary in gene content rather than allelic polymorphism and therefore seem to execute more specialized functions and/or might be involved in the recognition of conserved ligands and peptides [7, 29, 44].

For humans, KIR alleles are also distinguished by SNPs in their introns (IPD-KIR, release 2.9.0) [65], which might impact, for instance, the expression level and posttranscriptional splicing. A total of 353 human KIR alleles can only be distinguished from the reference gene based on intronic variations (IPD-KIR, release 2.9.0), and this 
number is likely to be underestimated [65]. Sequence data on the non-coding KIR gene regions are lacking for non-human primate species, but a similar extent of intronic variations might be feasible and may impact their receptor functionality. However, there are no disease or health associations reported for intronic polymorphisms within the KIR genes, but abundant pathological conditions are described for intronic variations in many other genes mapping elsewhere in the genome [66]. For example, a SNP in the human CYP2D6 gene is linked to a decreased expression of the functional transcript and correlates with a lower metabolic activity [67]. For HLA-DP, a single nucleotide variation in the $3^{\prime}$ UTR modulates the expression level of different allotypes, which impacts the susceptibility to chronic hepatitis B virus infection [68].

Allele variation is mainly generated by synonymous and non-synonymous point mutations, and only the latter ones will impact the composition of the gene products. In sharp contrast to $\mathrm{MHC}$ class I polymorphisms, the allelic nucleotide variations of the $K I R$ genes are evenly distributed over the coding regions. The high concentration of $\mathrm{CpG}$ islands located in the KIR gene cluster might contribute to an elevated mutation rate, as these islands are in general more prone to promote nucleotide transitions [6971]. In addition, chromosomal rearrangements are known as mutagenic events [69, 72-74]. In particular, the regions that surround genomic insertions and deletions display an increased mutation rate, which might be induced by error-prone DNA replication [69, 75-77]. The abundant recombination that is accompanied by insertions and deletions in the primate KIR cluster is likely to contribute to the extensive allelic KIR variation. Within two and three generations of human and macaque families studied, the birth of novel KIR alleles is described, which might further substantiate the rapid mutation rate in this gene cluster $[29,78]$. To our knowledge, such an event has not been recorded for the highly polymorphic MHC class / genes.

The variation involving KIR genes at the allele level impacts the interactions with their highly polymorphic MHC class I ligands, and demonstrate that point mutations contribute to a diversified $K I R$ gene system. The general lack of allele level characterization in the clinic might limit the number of associations reported for KIR allele heterogeneity and their functional and disease-related effects. Even intronic variations might impact the KIR receptor expression and function. These few associations highlight the need to further characterize the KIR gene content of humans and other primate species at an allele level resolution.

\section{Alternative splicing as a mechanism for structural diversification}

The complexity of the primate KIR gene cluster is further extended by alternative splicing (Figure 2C) [79-83]. This post-transcriptional mechanism can generate multiple messenger RNA (mRNA) transcripts from a single gene, which are translated into different receptor isoforms. Constitutive splicing excludes the intronic sequences from the precursor mRNA (pre-mRNA) and ligates the coding exons. Alternative splicing 
deviates from this pattern by the use of alternative splice sites, the skipping of exons, and the retention of introns (Figure 2C) [84]. The alternative splice events for human and macaque KIR transcripts are well documented, and demonstrated that both inand out-of-frame transcripts are generated [79-83]. The out-of-frame transcripts often have an early stop codon, and this results in early truncation of the transcript. Even though these out-of-frame transcripts appear as a redundant side effect of alternative splicing, it might reflect a regulatory pathway to rapidly downregulate receptor expression. The functional impact of the in-frame generated KIR isoforms may be diverse. The skipping of exons generates transcripts that encode modified KIR isoforms, which lack one or two extracellular domains, the stem region, or the transmembrane region. These KIR isoforms probably exhibit differential binding properties or are secreted as soluble receptors (Figure $\mathbf{2 C}$ ) [85]. In-frame splice events that involve alternative splice sites might insert a partial intronic sequence into the transcript or delete a part of a coding exon. Although the functional and structural consequences of these KIR isoforms are harder to predict, they are likely to modify the receptor expression level, cellular localization, and ligand interactions.

Several splice events were frequently recorded or were defined for multiple KIR genes, and implicate the existence of conserved splice events that generate structurally and functionally distinct isoforms. For example, exon 4 (coding for the D1 domain) is frequently skipped from KIR3DL20 transcripts in macaques, thereby generating transcripts that encode both the complete receptor and receptors with a D0-D2 domain configuration $[43,57]$. This macaque isoform is termed KIR2DLO5, as it displays an $89.5 \%$ sequence similarity with human KIR2DL5. Moreover, it demonstrates that alternative splicing expands the macaque KIR repertoire by generating a second twodomain structure (KIR2DL) additional to KIR2DL04. The most frequent KIR splice event in humans involved the skipping of exon 6 , which encodes the stem region. Other frequent events included the skipping of exon 5 (D2 domain) and partial deletions in exons 4 and 5. These events result in isoforms that are likely to display altered binding properties, but their exact activity and localization remains elusive. Another common splice event in humans might function as a regulatory switch for expression of the $9 \mathrm{~A}$ and 10A KIR2DL4 alleles by restoring or disrupting the open reading frame [79]. Less frequent alternative splicing events were often found to be gene specific, and were mainly out-of-frame events that encoded for truncated receptors. Except for most exon skipping events, only a single splice event was shared between humans and macaques. This event involved a partial deletion of exon 3 (D0 domain) mediated by an alternative 5' splice site [79]. Data on the alternative splicing in other hominoids are lacking, but a similar extent of alternative splicing is likely to diversify their KIR receptors and repertoire.

The splicing of pre-mRNA not only facilitates diversification of the KIR repertoire, but might also compensate for genomic alterations that result in out-of-frame transcripts. The expression of human and macaque lineage III KIR genes, for example, requires the constitutive skipping of exon 3 to maintain an open reading frame (ORF). This exon contains a deletion of $5 \mathrm{bp}$ at the genomic DNA level, which would shift the 
reading frame that introduces an early stop codon $[79,86]$. The constitutive skipping of exon 3 suggests that the expanded repertoire of human KIR2D receptors evolved from a KIR3D gene. The absence of a conserved 33 bp sequence in intron 2 of all human and macaque lineage III KIR genes might relate to the constitutive exon skipping by, for example, disrupting the spliceosome recognition site [79].

The extensive levels of alternative splicing observed in humans and macaques defines another layer of complexity for the KIR gene cluster. This diversifying mechanism generates structurally and functionally distinct receptor isoforms, and might be involved in the regulation of receptor expression levels. Although not all isoforms might be functional, the frequency and consistency of several alternative splicing events suggest that alternative splicing is a rapid mechanism to diversify the KIR content in hominoids and Old World monkeys.

\section{Differential NK cell populations due to variegated KIR gene expression}

KIR gene plasticity is further reflected by the stochastic expression of a subset of KIR genes from the total gene repertoire in individual NK cells (Figure 2D). This selective transcriptional activation generates specialized NK cell populations, which express different numbers and combinations of KIR genes [87, 88]. The stochastic KIR expression is activated during NK cell maturation, and the transcriptional pattern is maintained by the methylation of silenced $K I R$ genes $[89,90]$. The different KIR receptor combinations are generated largely at random, but might be shaped by the individual KIR gene frequencies and the MHC class I repertoire. Therefore, KIR genes that are present on both chromosomes in heterozygous individuals, or genes that are present as two or more allotypes on a single haplotype (e.g., by duplication or gene insertion), could be expressed in a mono- and multi-allelic manner. This may generate NK cell subsets that transcribe two or more allelic copies of a certain KIR gene [28]. Divergent expression patterns are documented for human KIR2DL4, which is expressed in all NK cells, and for KIR3DL3, which is expressed at low levels [91, 92].

The molecular regulation of KIR gene expression is well studied in humans, and involves multiple promoter regions in the intergenic sequences that control gene demethylation and transcription [27, 91-97]. The proximal promoter is located directly in front of the first exon of a KIR gene and functions as a probabilistic switch (Figure 2D). Bi-directional transcription of this promoter generates forward and reverse transcripts that correlate with the activation and suppression of $K I R$ gene transcription, respectively. Forward transcripts of a distal promoter are associated with activation of the proximal promoter region and appear to be required for eventual KIR gene expression. A third promoter upstream of the proximal promoter, also denoted as the intermediate promoter, modulates the bidirectional transcription of the proximal promoter directly or indirectly by mediating correct splicing of the forward proximal promoter transcripts $[27,95]$. In all human KIR genes, the promoter regions are highly 
conserved, with 91-99.6\% sequence similarity. Exceptions are found for the promoters of KIR2DL4 and KIR3DL3, which substantiates their diverged expression profile [95]. Three types of promoter regions are defined for human KIR2DL5, which display considerable differences in their nucleotide sequence and transcription factor binding sites. Types I and III control variegated expression, whereas transcripts of KIR2DL5 alleles that exhibit the type II promoter are undetectable $[98,99]$. These type II promoters are probably inactivated by a SNP in their Runt-related transcription factor (RUNX) transcription binding site, which is an important motif in the regulation of gene expression, and is generally conserved in all KIR genes [99]. An identical SNP is identified in the proximal promoter of the pseudogene KIR3DP1, and might indicate that the inactive type II promoter is swapped to particular KIR2DL5 alleles by chromosomal recombination [99-101]. Within the KIR promoter regions, multiple other transcription factor binding sites are identified, which can vary per KIR gene and thereby contribute to differential gene expression. Allelic variations of the different transcription factor binding sites modulate the expression levels of $K I R$ alleles [27, 93]. For example, a KIR2DL1 allele displayed low expression, which was associated with three SNPs in the distal promoter that generated a binding site for the Zinc finger E-box-binding homeobox 1 (ZEB1) protein [27]. This transcription factor is associated with the downregulation of IL2 expression, and might have a similar impact on the expression of this specific KIR2DL1 allele. Just like the variation in the KIR gene introns, the nucleotide polymorphisms in the promoter regions are grossly undervalued, despite the direct impact on the expression of KIR alleles.

The variegated expression pattern of the KIR genes defines NK cell subsets, of which several are tissue resident. These NK cell populations might execute specialized functions in particular tissues that could be mediated by specific sets of KIR receptors. For example, the KIR expression profile of NK cells that were derived from the lung, liver, and uterus deviates from the expression pattern observed in peripheral blood NK cells [102-104]. Expression of KIR was also established for subsets of T cells, in particular terminally differentiated CD8+ T cells, of which 30\% exhibited KIR expression [105107]. The majority of these T cells dominantly express a single inhibitory or activating $K I R$ gene, which is generally distinct from the KIR gene expression pattern on NK cells within the same individual [105]. The expression pattern of NK cells and CD8+ T cells can be erased by in vitro treatment with a methylation inhibitor (5-azacytidine), and thereby induce the expression of formerly silenced KIR genes [97, 108, 109]. This demonstrates that the stochastic KIR gene expression is maintained by methylation in both types of lymphocytes.

The variability in the promoter regions that is mainly generated by point mutations and chromosomal recombination events contributes to the diversification of NK cell subsets by the stochastic methylation of KIR genes. The promoter regions and epigenetic regulation of the $K I R$ gene cluster in non-human primate species are less well characterized, but their stochastic expression pattern indicates a similar genetic mechanism. 


\section{The different characters of diversification in the $K I R$ and $M H C$ clusters}

The expansion of the primate KIR cluster was probably initiated by the integration of multiple retroviral elements near or in the founding KIR genes. Subsequent duplications were mediated by these transposable elements, and this process had an impact on the expansion of the KIR gene repertoire [35]. These recombination events might have enhanced the mutation rate within this genomic region that generated a diverse set of KIR alleles, and subsequently some of these were positively selected during evolution. In the case of exons, the point mutations may affect the receptor structure, function, localization, and expression, whereas polymorphisms in the introns may enhance the level of alternative splicing by affecting existing or generating alternative splice sites. In addition, the high level of point mutations caused variation within the promoter regions, and thereby modulated the variegated expression pattern and expression level of KIR receptors. It appears that all the different molecular mechanisms are intertwined and enhanced by each other, which multiplies their diversifying impact on the primate $K I R$ gene system.

The $M H C$ class I gene family is considered one of the most polymorphic genomic regions in primates, but displays a different nature of diversity as compared to its KIR ligands. In hominoids, the fixed number of $M H C-A,-B$, and $-C$ genes on a haplotype indicate low levels of recent duplications and chromosomal recombination, which is substantiated by an exceptionally low recombination rate for the MHC class I region $[110,111]$. Chromosomal rearrangements that are accompanied by the formation of an MHC class I fusion gene, as is determined for the KIR genes, is to our knowledge not known. In most hominoids, MHC class I polymorphism is mainly generated by point mutations in concert with a recombination of small segments. These genetic modifications are especially located in the exons encoding the peptide-binding site, and indicate a rigorous selection for a diverse array of allotypes. The functional impact is reflected in differential peptide presentation [18]. Additional modification of the $\mathrm{MHC}$ repertoire is reflected at the transcription level by alternative splicing, which is reported for human and macaque MHC transcripts [112-116]. Considering the high level of allelic polymorphism in the HLA genes, which may involve nucleotide substitutions that disrupt existing or generate novel alternative splice sites, one might expect abundant alternative splicing events in their transcripts. However, only a modest level of alternative splicing is demonstrated for several classical and nonclassical HLA class I alleles, which mainly involved exon skipping that abrogated receptor surface expression [112]. Specific isoforms of the non-classical HLA-G, however, are well known and are associated with cancer and inflammatory diseases [117-120]. In contrast, alternative splicing in primate KIR was not limited to certain alleles, and also comprised conserved splice events that were common to multiple KIR genes and lineages [79]. The classical MHC class I allotypes are constitutively expressed on all nucleated cells, and thereby lack a variegated expression pattern [121, 122]. However, individual MHC allotypes may display a differential expression level, which is 
affected by sequence variation, tissue distribution, and pathogenic encounters [122, 123]. In humans, the relative surface expression of HLA-A and-B is approximately ten times higher compared to HLA-C molecules $[122,124]$. This suggests that the HLA-C gene might slowly shift its main function from classical antigen presentation into the modulation of NK cell responses during infection and reproductive biology. In addition, the expression levels of different HLA-C alleles display variation, in which highly expressed allotypes correlated with a beneficial control of HIV infection [125]. The differential expression pattern is also determined for the expanded $\mathrm{MHC}$ class I region in macaques, with only a few highly expressed MHC-A and-B allotypes [126, 127]. The $\mathrm{MHC}$ expression levels are, however, not strictly maintained and can be modulated during infection by immune regulators such as interferon and tumor necrosis factor (TNF) [122].

The primate KIR and $M H C$ gene families are both reflected by great complexity, and seem to co-evolve to maintain a functional relationship. The MHC class / diversification mainly involved allelic polymorphism in the exons encoding the peptide binding site and recombination of small segments, which is driven by the arms race with rapidly evolving pathogens. The KIR genes, in contrast, are diverged by haplotype expansion and contraction, random point mutations, and the generation of novel fusion genes. The expression and structural variability of the KIR receptors are further modified at the epigenetic and post-transcriptional level, whereas a similar diversification of the $\mathrm{MHC}$ class I molecules is limited. The conjunction of different genetic mechanisms generates an extensive plasticity for the primate KIR gene cluster, which seems to exceed the diversity of the polymorphic MHC class / genes.

\section{CD94:NKG2A- or KIR-dependent education in different primate species}

A comparison of the KIR gene system in primate species illustrates a variable degree of gene expansion, reflected in the differential expansion of gene lineages (Figure 1). This might be largely due to co-evolution with their diverse MHC class I repertoire. The variable extent of expansion, however, is emphasized by the number of functional genes per KIR haplotype and by the overall size of the KIR gene repertoire documented for a certain primate species. The extremes are represented by the heavily contracted KIR haplotypes in bonobos versus the widely expanded set of $K I R$ genes in macaques (Figure 1). The flexibility to expand and contract $K I R$ haplotypes and repertoires, apparently without compromising sufficient and protective immune responses, might be closely related to the nature of NK cell education in different primate species.

NK cells require self-tolerance and a signal to activate, which are acquired through an educational process. NK cell education involves the recognition of self-MHC class I molecules or the presented peptides by at least one inhibitory NK cell receptor. Alternative educational pathways that are MHC-independent are reported, but their exact contribution to the acquiring of NK cell functions is elusive $[128,129]$. The MHC- 
dependent education is predominant and can be approached in two ways (Figure 3) $[13,130,131]$. One strategy of NK cell education involves the interaction of inhibitory CD94:NKG2A NK cell receptors with the non-polymorphic MHC-E molecules, which are complexed with conserved signal peptides derived from the diversified classical MHC class I molecules [132-134]. One could argue that this approach allows the immune system to scan in a crude way whether total MHC class I expression has been abrogated. In the complementary approach, however, NK cell education is established through interaction of the MHC class I molecules with polymorphic KIR receptors. This seems to reflect a more sophisticated strategy in which the immune system checks at the epitope level for a malfunctioning of MHC class I expression. KIR-dependent NK cell education is mainly conducted through the interactions of inhibitory KIR and MHC

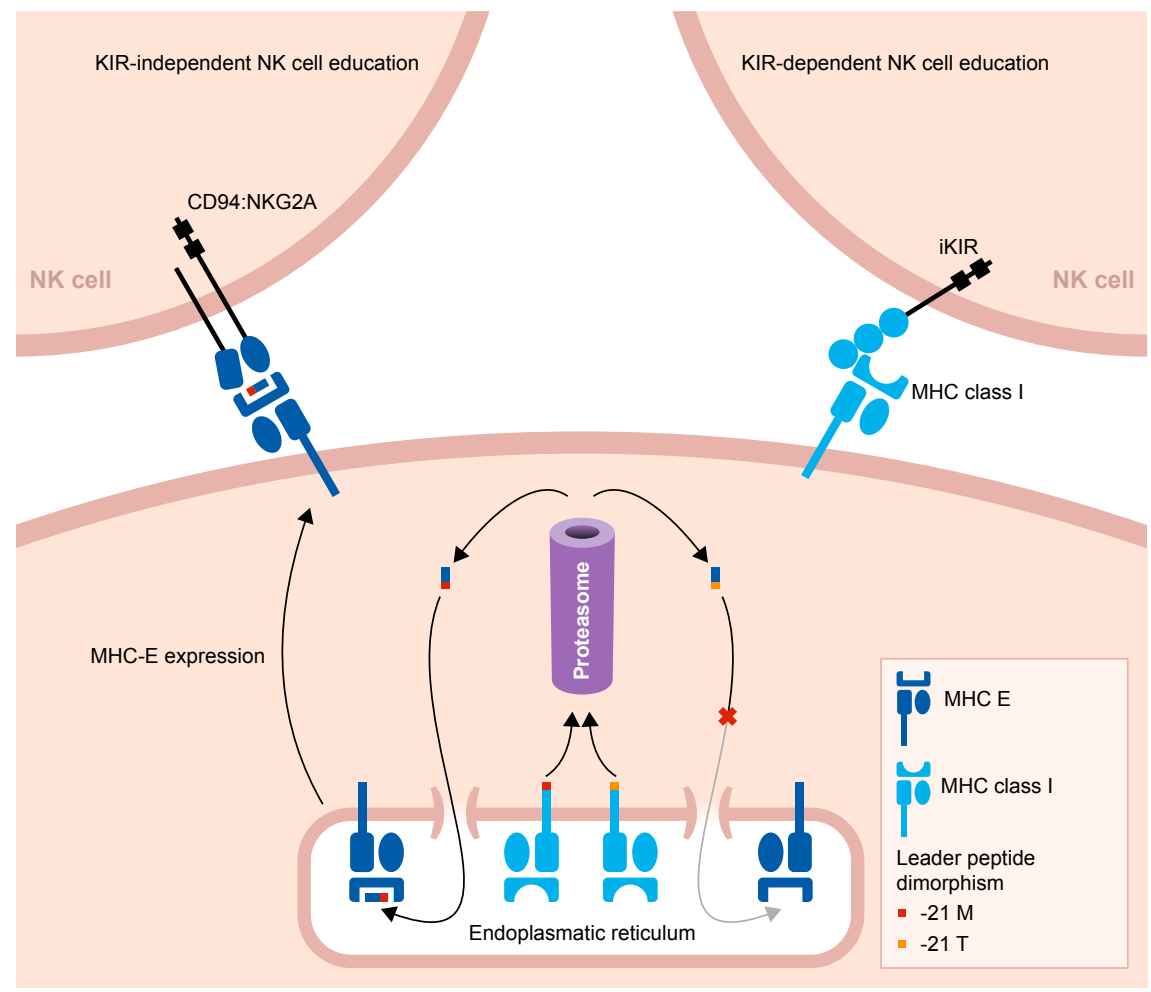

Figure 3. Two pathways to educate NK cells in primates. A schematic overview of two strategies to educate NK cells. The leader peptide of MHC class I molecules either contains a methionine $(-21 \mathrm{M})$ or threonine $(-21 \mathrm{~T})$ residue. The-21M peptides strongly bind to MHC-E molecules, and these complexes display an increased cell surface level. Through the conserved CD94:NKG2A receptors, the MHC-E complexes educate and license NK cells. In contrast,-21T leader peptides, which are predominantly present in MHC-B allotypes of humans and chimpanzees, do not interact with MHC-E molecules. Therefore, in the presence of one or more-21T MHC class I allotypes, the cell surface level of MHC-E is decreased. In this case, NK cells are educated by MHC class I molecules that interact with their KIR ligands. 
class I molecules. However, activating KIR contribute to the tuning of NK cell responsiveness by dampening NK cell activity upon MHC class I recognition [135]. Currently, only for KIR2DS1 the effect on NK cell education is described. In the following sections, we mainly consider the educational impact of inhibitory KIR.

Whether the NK cells are educated by the CD94:NKG2A or KIR pathway might depend on a single nucleotide dimorphism at position 21 of the $\mathrm{MHC}$ class I leader sequences. Most MHC-A and $-\mathrm{C}$ molecules in hominoids have a methionine (-21M) residue present at this position, whereas in general this position is occupied by threonine (-21T) in MHC-B molecules. The $-21 \mathrm{M}$ peptides strongly bind to MHC-E molecules and promote cell surface expression of MHC-E complexes [136]. The presence of five or six classical MHC class I allotypes containing the-21M residue drives the NK cell education towards the more conserved MHC-E and CD94:NKG2A interactions. However, approximately $62 \%$ of human individuals display a-21T HLA-B homozygous genotype, with a variable distribution in different populations [130]. In chimpanzees, $-21 T$ is near fixed in their MHC-B allotypes [13]. The homozygous threonine genotype corresponds with a low MHC-E surface expression. As a consequence, human and chimpanzee NK cells are largely educated by their KIR repertoire $[13,130]$. In contrast, in macaque MHC-A and-B allotypes, methionine is the predominant residue at position 21 of the leader sequence, which results in an NK cell education that mostly relies on the conserved CD94:NKG2A pathway [130].

In primate species with a KIR-dependent NK cell education, one can envision that an expanded KIR repertoire may compromise NK cell activity. This might drive selection for a limited KIR expansion, as we will discuss in the next section. If this reasoning is true, the KIR-independent education of NK cells in macaques might result in an extensive expansion of their KIR gene system. We think that the primary function of macaque KIR is focused on the recognition and elimination of infected or malignant cells. This defense mechanism relies on the recognition of Bw4 and Bw6 epitopes, but KIR interactions are also sensitive to non-self peptides that can be presented by MHC class I molecules [137-141]. A large genetic diversity of KIR genes provides a broader repertoire to scan all the variable MHC class I allotypes in combination with their peptides originating from pathogens. It has been proposed that up to seven distinct KIR receptors are required for successful peptide recognition [142]. This optimal receptor count might even be higher when the Bw4 and Bw6 epitope specificity is considered for the different KIR allotypes. The high level of chromosomal recombination and the relatively frequent formation of fusion genes in macaques might indicate selection for a widely diversified KIR gene system. Considering their KIR-independent NK cell education, KIR expansion in macaques might be exempted from potential negative selection on large KIR gene repertoires.

However, not all macaque KIR haplotypes contain a large number of genes, and they even display indications for contraction by chromosomal recombination events. The formation of novel gene entities by the shuffling of head- and tail-encoding exons is achieved by recombination events, which are coherently accompanied both by contractions and expansions of KIR haplotypes. There might be a trade-off between 
the expansion of the overall KIR repertoire in a population by generating fusion genes and the contraction of KIR haplotypes in individuals. Rapid expansion and diversification generate a highly plastic macaque KIR gene system that appears to be maintained by selection to militate against rapidly evolving pathogens.

\section{KIR haplotype expansion and contraction: finding the equilibrium}

As compared to macaques, hominoids appear to have a more limited haplotype content and overall KIR repertoire (Figure 1, Table I). These limitations might be maintained by selective pressure on an efficient KIR-dependent NK cell education, but should be balanced with protection against infections. This balance might be reflected in the slightly variable KIR gene content per haplotype.

A large KIR repertoire is likely to provide a broad array of MHC class I specificities that may result in the education of an increased fraction of NK cells (Figure 4A). Moreover, the expression of multiple self-specific inhibitory KIR receptors by NK cell clones enhances the magnitude of their effector response [143]. Although only a small population of NK cells dominantly expresses more than one inhibitory KIR receptor, an expanded KIR repertoire might enlarge this NK cell population size and elevate the strength of the NK cell response (Figure $\mathbf{4 A}$ and $\mathbf{4 C}$ ). A potential detrimental effect of an expanded KIR haplotype might emerge if the repertoire comprises only a few or abundant self-specific receptors. On the one hand, the variegated expression of a large KIR repertoire that consists of few self-specific receptors might thin out the educated NK cell population and provide an inefficient immune surveillance (Figure 4B). Indications for a biased expression of self-specific KIR suggest modulation of the KIR expression by an individual's MHC class I repertoire [144-146], which would ensure a more robust immune response and might compensate for a large non-self-specific KIR expansion. On the other hand, a large repertoire of self-specific KIR might enlarge the fraction of educated NK cells that display increased activity, which might be protective in infections and cancer (Figure 4C). However, elevated NK cell activity, which might be further enhanced by the expression of multiple self-specific KIR on NK cell subsets, or excessive NK cell inhibition by abundant self-specific KIR interactions are also associated with implantation failure and recurrent miscarriages [147-150]. Furthermore, overactivation might desensitize NK cells and result in hyporeactivity [151], which might weaken subsequent immune responses. Therefore, a large KIR repertoire that is used in NK cell education might act as a double-edged sword that can both enhance and compromise an individual's immune response.

In contrast, individuals that have a limited KIR haplotype rely on only one or few self-specific KIR receptors to educate their NK cells (Figure 4D). Even though a sufficient percentage of NK cells might be educated by a limited KIR repertoire, the specificity is restricted, and specialized NK cell populations might lack. The complete absence of NK cell education occurs in MHC class I-deficient mice, which display a near normal NK cell 
count with an overall reduced responsiveness $[152,153]$. In humans and other hominoid species, individuals that completely lack self-specific KIR are not documented. This indicates that even minimal KIR haplotypes provide education, and suggests that framework KIR receptors could play a substantial role in the NK cell education of hominoids. In addition, the chance that an individual completely lacks self-specific KIR receptors is reduced by the heterozygous nature of the KIR gene cluster. As far as we know, only few human and no non-human primate individuals are documented that were homozygous for their KIR haplotypes at an allele level [154]. In a rhesus macaque family studied, one individual was assumed to be KIR-homozygous according to segregation. However, more detailed analysis illustrated that one KIR gene copy appeared to have gained point mutations that resulted in the haplotypes diverging at an allele level [29]. This individual macaque possessed a largely homozygous KIR
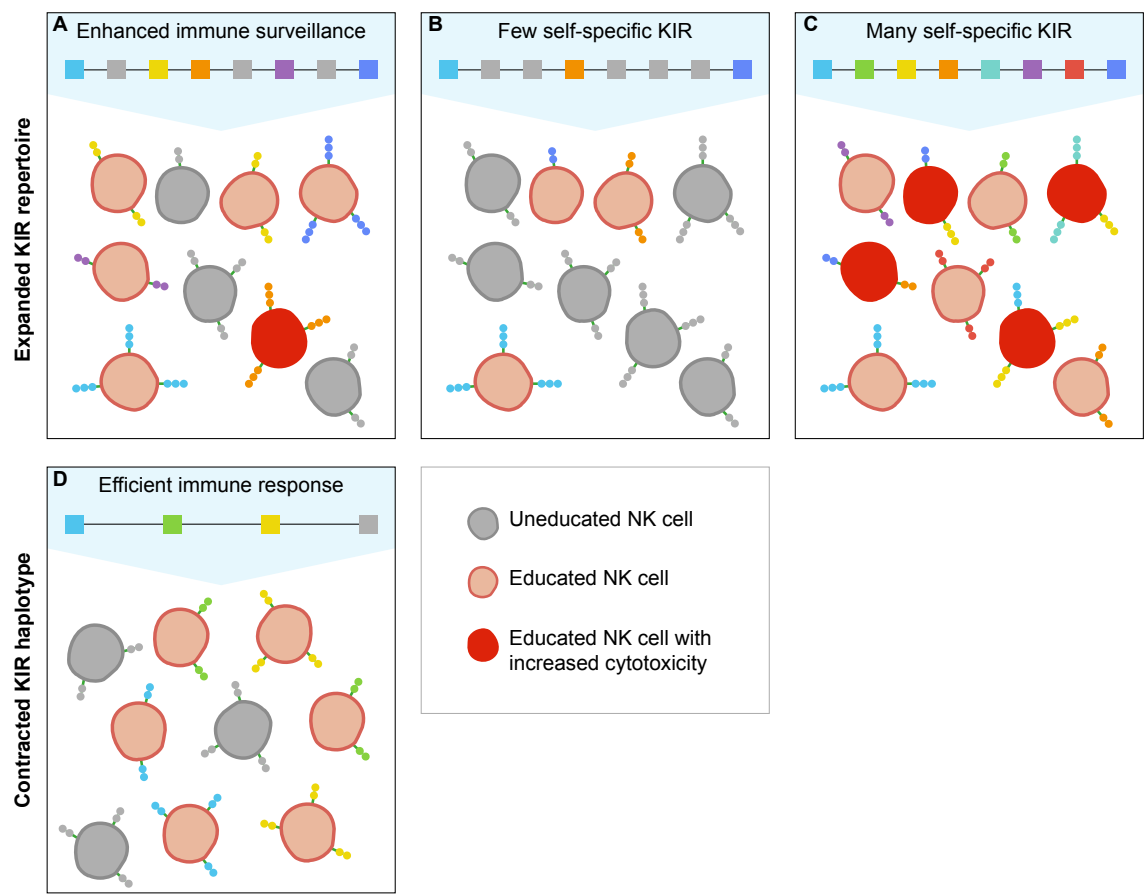

Figure 4. The education of NK cells by expanded and contracted KIR haplotypes. The proposed populations of NK cells that are educated (red cells) by self-specific KIR (colored boxes and receptors) on expanded and contracted haplotypes. The grey boxes represent non-self-specific KIR, and are displayed on the uneducated NK cell clones (grey cells). (A) An expanded KIR haplotype provides a broad MHC class I specificity, and might educate some NK cell clones through multiple self-specific receptors, which increases their cytotoxicity (dark red cells). (B) A large KIR repertoire with only a few self-specific KIR might lower the fraction of educated NK cells and thereby provide an insufficient immune response, (C) whereas abundant self-specific receptors increase the educated fraction and their cytotoxicity. (D) A small KIR repertoire might educate large fractions of NK cells with a limited MHC class I specificity, which might provide largely sufficient immune responses. 
content, but did not display an impaired immune system; it also produced healthy offspring, which suggests that KIR-heterozygosity is not vital. However, KIR haplotype diversity might compensate for limited KIR haplotypes and improve the immune surveillance, as is also described for MHC heterozygosity [155-157].

In contrast to non-self-specific T lymphocytes, which are depleted upon a failed positive or negative selection in the thymus, uneducated NK cells are present in the peripheral blood. The relatively high level of uneducated NK cells in individuals with small or large non-self-specific KIR repertoires could affect their immune surveillance, but does not preclude an efficient immune response during infection or tumor formation. In fact, unlicensed NK cells appear to be more efficient at eradicating infected or malignant cells that persistently express MHC class I molecules or viral mimic ligands through their reactivation by cytokines or NKG2D receptors [158-160]. Therefore, a fraction of uneducated NK cells in combination with a largely educated NK cell population might be more protective than a completely educated NK cell pool with broad MHC class / specificity.

There could be another factor, however, that limits expansion of the KIR haplotypes and gene repertoire, in addition to their role in NK cell education. In orangutans, MHC-B allotypes contain a-21M leader peptide, which would suggest education via the conserved CD94:NKG2A pathway [130]. In contrast to macaques, the orangutan KIR system is not extensively expanded, and is more in line with other hominoids that display a KIR-dependent NK cell education. The emergence of MHC-C as a specialized ligand for KIR might override the dimorphism and coherent increase in MHC-E expression, and drive NK cell education via the KIR receptors. In addition, the number of characterized MHC-B molecules in orangutans is relatively low (IPD-MHC, release 3.4.0.1) [161]. A larger sample group of orangutans or additional functional studies would be required to test our hypothesis for the differential KIR expansion in primate species that exert a KIR-independent or-dependent NK cell education.

Nevertheless, the diverse KIR haplotype content and overall gene repertoire in hominoids and Old World monkeys are likely to affect the education, activity, and function of their NK cells, but the precise effect of the haplotype expansions and contractions remains ambiguous. The equal distribution of both small and large KIR repertoires in humans and macaques indicates a balancing selection, which might be an ongoing process to achieve a haplotype equilibrium that serves differential functions, such as fighting infections and promoting successful pregnancy. 


\section{Conclusion}

The KIR gene system is well studied in humans, and reveals multiple mechanisms that contribute to the plasticity of this immunogenetic cluster (Figure 2). In other hominoid species, such as chimpanzees and orangutans, indications for a similar diversifying genetic toolset is evident, although robust data on some mechanisms are lacking, such as alternative splicing and variegated expression. The variability of the extensively diversified KIR gene cluster in macaques exceeds that observed in hominoids, with a prominent expansion of the lineage II KIR genes, which is largely mediated by recombination events. The rapid evolution of the KIR gene cluster may counteract the adaptive nature of pathogens. The species-specific diversification of the $K I R$ gene cluster might be largely driven by co-evolution with their diversified MHC class I repertoire and thereby indirectly by the arms race with pathogens. In addition, a KIR-dependent or-independent NK cell education might impact the variable haplotype content and the extent of KIR gene expansion. Nevertheless, the different molecular mechanisms responsible for diversification of the KIR gene cluster are shared in Old World monkeys and hominoids, which suggests an evolutionary effort to diversify the KIR gene system.

\section{Acknowledgements}

We thank D. Devine for editing the manuscript and F. van Hassel for preparing the figures. 


\section{References}

1. Ljunggren HG, Karre K: In search of the 'missing self': MHC molecules and NK cell recognition. Immunology today 1990, 11(7):237-244.

2. Vivier E, Tomasello E, Baratin M, Walzer T, Ugolini S: Functions of natural killer cells. Nature immunology 2008, 9(5):503-510.

3. Björkström NK, Riese P, Heuts F, Andersson S, Fauriat C, Ivarsson MA, Björklund AT, Flodström-Tullberg M, Michaëlsson J, Rottenberg ME et al: Expression patterns of NKG2A, KIR, and CD57 define a process of CD56dim NK-cell differentiation uncoupled from NK-cell education. Blood 2010, 116(19):3853-3864.

4. Brodin P, Karre K, Hoglund P: NK cell education: not an on-off switch but a tunable rheostat. Trends in immunology 2009, 30(4):143-149.

5. Kelley J, Walter L, Trowsdale J: Comparative genomics of natural killer cell receptor gene clusters. PLoS genetics 2005, 1(2):129-139.

6. Martin AM, Freitas EM, Witt CS, Christiansen FT: The genomic organization and evolution of the natural killer immunoglobulin-like receptor (KIR) gene cluster. Immunogenetics 2000, 51(4-5):268-280.

7. Bruijnesteijn J, de Groot NG, Otting N, Maccari G, Guethlein LA, Robinson J, Marsh SGE, Walter L, O'Connor DH, Hammond JA et al: Nomenclature report for killer-cell immunoglobulin-like receptors (KIR) in macaque species: new genes/alleles, renaming recombinant entities and IPD-NHKIR updates. Immunogenetics 2020, 72(1):37-47.

8. Marsh SG, Parham P, Dupont B, Geraghty DE, Trowsdale J, Middleton D, Vilches C, Carrington M, Witt C, Guethlein LA et al: Killer-cell immunoglobulin-like receptor (KIR) nomenclature report, 2002. Immunogenetics 2003, 55(4):220-226.

9. Robinson J, Guethlein LA, Maccari G, Blokhuis J, Bimber BN, de Groot NG, Sanderson ND, Abi-Rached L, Walter L, Bontrop RE et al: Nomenclature for the KIR of non-human species. Immunogenetics 2018, 70(9):571-583.

10. Sambrook JG, Bashirova A, Andersen H, Piatak M, Vernikos GS, Coggill P, Lifson JD, Carrington M, Beck S: Identification of the ancestral killer immunoglobulin-like receptor gene in primates. BMC Genomics 2006, 7(1):209.

11. Sanderson ND, Norman PJ, Guethlein LA, Ellis SA, Williams C, Breen M, Park SD, Magee DA, Babrzadeh F, Warry A et al: Definition of the cattle killer cell Ig-like receptor gene family: comparison with aurochs and human counterparts. Journal of immunology (Baltimore, Md : 1950) 2014, 193(12):6016-6030.

12. Guethlein LA, Abi-Rached L, Hammond JA, Parham P: The expanded cattle KIR genes are orthologous to the conserved single-copy KIR3DX1 gene of primates. Immunogenetics 2007, 59(6):517-522.

13. Wroblewski EE, Parham P, Guethlein LA: Two to Tango: Co-evolution of Hominid Natural Killer Cell Receptors and MHC. Frontiers in immunology 2019, 10:177.

14. de Groot NG, Heijmans CMC, van der Wiel MKH, Blokhuis JH, Mulder A, Guethlein LA, Doxiadis GGM, Claas FHJ, Parham P, Bontrop RE: Complex MHC Class I Gene Transcription Profiles and Their Functional Impact in Orangutans. The Journal of Immunology 2016, 196(2):750.

15. Karl JA, Bohn PS, Wiseman RW, Nimityongskul FA, Lank SM, Starrett GJ, O'Connor DH: Major histocompatibility complex class I haplotype diversity in Chinese rhesus macaques. G3 (Bethesda) 2013, 3(7):1195-1201.

16. Doxiadis GGM, de Groot N, Otting N, Blokhuis JH, Bontrop RE: Genomic plasticity of the MHC class I A region in rhesus macaques: extensive haplotype diversity at the population level as revealed by microsatellites. Immunogenetics 2011, 63(2):73-83.

17. Daza-Vamenta R, Glusman G, Rowen L, Guthrie B, Geraghty DE: Genetic divergence of the rhesus macaque major histocompatibility complex. Genome Res 2004, 14(8):1501-1515.

18. de Groot NG, Blokhuis JH, Otting N, Doxiadis GG, Bontrop RE: Co-evolution of the MHC class I and KIR gene families in rhesus macaques: ancestry and plasticity. Immunological reviews 2015, 267(1):228-245.

19. Otting N, Heijmans CMC, Noort RC, de Groot NG, Doxiadis GGM, van Rood JJ, Watkins DI, Bontrop RE: Unparalleled complexity of the MHC class I region in rhesus macaques. Proc Natl Acad Sci U S A 2005, 102(5):1626.

20. Wiseman RW, Karl JA, Bohn PS, Nimityongskul FA, Starrett GJ, O'Connor DH: Haplessly hoping: macaque major histocompatibility complex made easy. ILAR J 2013, 54(2):196-210. 
21. Schafer JL, Colantonio AD, Neidermyer WJ, Dudley DM, Connole M, O'Connor DH, Evans DT: KIR3DL01 recognition of Bw4 ligands in the rhesus macaque: maintenance of Bw4 specificity since the divergence of apes and Old World monkeys. Journal of immunology (Baltimore, Md : 1950) 2014, 192(4):1907-1917.

22. Parham P, Moffett A: Variable NK cell receptors and their MHC class I ligands in immunity, reproduction and human evolution. Nature reviews Immunology 2013, 13(2):133-144.

23. Rosner C, Kruse PH, Hermes M, Otto N, Walter L: Rhesus macaque inhibitory and activating KIR3D interact with Mamu-A-encoded ligands. Journal of immunology (Baltimore, Md : 1950) 2011, 186(4):2156-2163.

24. Bimber BN, Evans DT: The killer-cell immunoglobulin-like receptors of macaques. Immunological reviews 2015, 267(1):246-258.

25. Hermes M, Weil S, Groth A, Dressel R, Koch J, Walter L: Characterisation of mouse monoclonal antibodies against rhesus macaque killer immunoglobulin-like receptors KIR3D. Immunogenetics 2012, 64(11):845-848.

26. Moreland AJ, Guethlein LA, Reeves RK, Broman KW, Johnson RP, Parham P, O'Connor DH, Bimber BN: Characterization of killer immunoglobulin-like receptor genetics and comprehensive genotyping by pyrosequencing in rhesus macaques. BMC Genomics 2011, 12:295.

27. Wright PW, Li H, Huehn A, O'Connor GM, Cooley S, Miller JS, Anderson SK: Characterization of a weakly expressed KIR2DL1 variant reveals a novel upstream promoter that controls KIR expression. Genes and immunity 2014, 15(7):440-448.

28. Chan HW, Kurago ZB, Stewart CA, Wilson MJ, Martin MP, Mace BE, Carrington M, Trowsdale J, Lutz CT: DNA methylation maintains allele-specific KIR gene expression in human natural killer cells. The Journal of experimental medicine 2003, 197(2):245-255.

29. Bruijnesteijn J, de Groot N, van der Wiel MKH, Otting N, de Vos-Rouweler AJM, de Groot NG, Bontrop RE: Unparalleled Rapid Evolution of KIR Genes in Rhesus and Cynomolgus Macaque Populations. Journal of immunology (Baltimore, Md : 1950) 2020.

30. Martin AM, Kulski JK, Witt C, Pontarotti P, Christiansen FT: Leukocyte Ig-like receptor complex (LRC) in mice and men. Trends in immunology 2002, 23(2):81-88.

31. Sambrook JG, Bashirova A, Palmer S, Sims S, Trowsdale J, Abi-Rached L, Parham P, Carrington M, Beck S: Single haplotype analysis demonstrates rapid evolution of the killer immunoglobulin-like receptor (KIR) loci in primates. Genome Res 2005, 15(1):25-35.

32. Wende $\mathrm{H}$, Colonna M, Ziegler A, Volz A: Organization of the leukocyte receptor cluster (LRC) on human chromosome 19q13.4. Mammalian genome : official journal of the International Mammalian Genome Society 1999, 10(2):154-160.

33. Trowsdale J, Knight JC: Major histocompatibility complex genomics and human disease. Annu Rev Genomics Hum Genet 2013, 14:301-323.

34. Traherne JA, Martin M, Ward R, Ohashi M, Pellett F, Gladman D, Middleton D, Carrington M, Trowsdale J: Mechanisms of copy number variation and hybrid gene formation in the KIR immune gene complex. Human molecular genetics 2010, 19(5):737-751.

35. Guethlein LA, Older Aguilar AM, Abi-Rached L, Parham P: Evolution of killer cell Ig-like receptor (KIR) genes: definition of an orangutan KIR haplotype reveals expansion of lineage III KIR associated with the emergence of MHC-C. Journal of immunology (Baltimore, Md : 1950) 2007, 179(1):491-504

36. Wilson MJ, Torkar M, Haude A, Milne S, Jones T, Sheer D, Beck S, Trowsdale J: Plasticity in the organization and sequences of human KIR/ILT gene families. Proceedings of the National Academy of Sciences 2000, 97(9):4778

37. Bailey JA, Liu G, Eichler EE: An Alu transposition model for the origin and expansion of human segmental duplications. American journal of human genetics 2003, 73(4):823-834.

38. Sen SK, Han K, Wang J, Lee J, Wang H, Callinan PA, Dyer M, Cordaux R, Liang P, Batzer MA: Human genomic deletions mediated by recombination between Alu elements. American journal of human genetics 2006, $79(1): 41-53$.

39. Han K, Lee J, Meyer TJ, Wang J, Sen SK, Srikanta D, Liang P, Batzer MA: Alu recombination-mediated structural deletions in the chimpanzee genome. PLoS genetics 2007, 3(10):1939-1949.

40. Roe D, Vierra-Green C, Pyo CW, Eng K, Hall R, Kuang R, Spellman S, Ranade S, Geraghty DE, Maiers M: Revealing complete complex KIR haplotypes phased by long-read sequencing technology. Genes and immunity 2017, 18(3):127-134. 
41. Rajalingam R, Gardiner CM, Canavez F, Vilches C, Parham P: Identification of seventeen novel KIR variants: fourteen of them from two non-Caucasian donors. Tissue antigens 2001, 57(1):22-31.

42. Pyo C-W, Wang R, Vu Q, Cereb N, Yang SY, Duh F-M, Wolinsky S, Martin MP, Carrington M, Geraghty DE: Recombinant structures expand and contract inter and intragenic diversification at the KIR locus. BMC Genomics 2013, 14(1):89.

43. Bruijnesteijn J, van der Wiel MKH, Swelsen WTN, Otting N, de Vos-Rouweler AJM, Elferink D, Doxiadis GG, Claas FHJ, Lardy NM, de Groot NG et al: Human and Rhesus Macaque \&lt;em\&gt;KIR\&lt;/em\&gt; Haplotypes Defined by Their Transcriptomes. The Journal of Immunology 2018, 200(5):1692.

44. Vierra-Green C, Roe D, Hou L, Hurley CK, Rajalingam R, Reed E, Lebedeva T, Yu N, Stewart M, Noreen H et al: Allele-Level Haplotype Frequencies and Pairwise Linkage Disequilibrium for 14 KIR Loci in 506 EuropeanAmerican Individuals. PLOS ONE 2012, 7(11):e47491.

45. Norman PJ, Abi-Rached L, Gendzekhadze K, Hammond JA, Moesta AK, Sharma D, Graef T, McQueen KL, Guethlein LA, Carrington CV et al: Meiotic recombination generates rich diversity in NK cell receptor genes, alleles, and haplotypes. Genome Res 2009, 19(5):757-769.

46. Leaton LA, Shortt J, Kichula KM, Tao S, Nemat-Gorgani N, Mentzer AJ, Oppenheimer SJ, Deng Z, Hollenbach JA, Gignoux CR et al: Conservation, Extensive Heterozygosity, and Convergence of Signaling Potential All Indicate a Critical Role for KIR3DL3 in Higher Primates. Frontiers in immunology 2019, 10(24).

47. Rajalingam R, Hong M, Adams EJ, Shum BP, Guethlein LA, Parham P: Short KIR haplotypes in pygmy chimpanzee (Bonobo) resemble the conserved framework of diverse human KIR haplotypes. The Journal of experimental medicine 2001, 193(1):135-146.

48. de Groot NG, Heijmans CMC, Helsen P, Otting N, Pereboom Z, Stevens JMG, Bontrop RE: Limited MHC class I intron 2 repertoire variation in bonobos. Immunogenetics 2017, 69(10):677-688.

49. Maibach V, Vigilant L: Reduced bonobo MHC class I diversity predicts a reduced viral peptide binding ability compared to chimpanzees. BMC Evolutionary Biology 2019, 19(1):14.

50. Wroblewski EE, Guethlein LA, Norman PJ, Li Y, Shaw CM, Han AS, Ndjango J-BN, Ahuka-Mundeke S, Georgiev AV, Peeters M et al: Bonobos Maintain Immune System Diversity with Three Functional Types of MHC-B. The Journal of Immunology 2017, 198(9):3480.

51. de Groot NG, Stevens JMG, Bontrop RE: Does the MHC Confer Protection against Malaria in Bonobos? Trends in immunology 2018, 39(10):768-771.

52. Gonzalez-Galarza FF, McCabe A, Santos E, Jones J, Takeshita L, Ortega-Rivera ND, Cid-Pavon GMD, Ramsbottom K, Ghattaoraya G, Alfirevic A et al: Allele frequency net database (AFND) 2020 update: gold-standard data classification, open access genotype data and new query tools. Nucleic Acids Res 2020, 48(D1):D783-d788.

53. Nakimuli A, Chazara O, Farrell L, Hiby SE, Tukwasibwe S, Knee O, Jayaraman J, Traherne JA, Elliott AM, Kaleebu $P$ et al: Killer cell immunoglobulin-like receptor (KIR) genes and their HLA-C ligands in a Ugandan population. Immunogenetics 2013, 65(11):765-775.

54. Abi-Rached L, Moesta AK, Rajalingam R, Guethlein LA, Parham P: Human-specific evolution and adaptation led to major qualitative differences in the variable receptors of human and chimpanzee natural killer cells. PLoS genetics 2010, 6(11):e1001192.

55. Guethlein LA, Norman PJ, Heijmans CMC, de Groot NG, Hilton HG, Babrzadeh F, Abi-Rached L, Bontrop RE, Parham P: Two Orangutan Species Have Evolved Different KIR Alleles and Haplotypes. Journal of immunology (Baltimore, Md : 1950) 2017, 198(8):3157-3169.

56. Macaca mulatta isolate AG07107 chromosome 19, whole genome shotgun sequence [https://www.ncbi.nlm. nih.gov/nuccore/CM014354]

57. Blokhuis JH, van der Wiel MK, Doxiadis GGM, Bontrop RE: The mosaic of KIR haplotypes in rhesus macaques. Immunogenetics 2010, 62(5):295-306.

58. VandenBussche CJ, Dakshanamurthy S, Posch PE, Hurley CK: A single polymorphism disrupts the killer Ig-like receptor 2DL2/2DL3 D1 domain. Journal of immunology (Baltimore, Md : 1950) 2006, 177(8):5347-5357.

59. Pando MJ, Gardiner CM, Gleimer M, McQueen KL, Parham P: The protein made from a common allele of KIR3DL1 (3DL1*004) is poorly expressed at cell surfaces due to substitution at positions 86 in Ig domain 0 and 182 in Ig domain 1. Journal of immunology (Baltimore, Md : 1950) 2003, 171(12):6640-6649. 
60. Frazier WR, Steiner N, Hou L, Dakshanamurthy S, Hurley CK: Allelic variation in KIR2DL3 generates a KIR2DL2like receptor with increased binding to its HLA-C ligand. Journal of immunology (Baltimore, Md: 1950) 2013, 190(12):6198-6208.

61. Hilton HG, Norman PJ, Nemat-Gorgani N, Goyos A, Hollenbach JA, Henn BM, Gignoux CR, Guethlein LA, Parham P: Loss and Gain of Natural Killer Cell Receptor Function in an African Hunter-Gatherer Population. PLoS genetics 2015, 11(8):e1005439.

62. Yawata M, Yawata N, Draghi M, Little AM, Partheniou F, Parham P: Roles for HLA and KIR polymorphisms in natural killer cell repertoire selection and modulation of effector function. The Journal of experimental medicine 2006, 203(3):633-645.

63. Martin MP, Qi Y, Gao X, Yamada E, Martin JN, Pereyra F, Colombo S, Brown EE, Shupert WL, Phair J et al: Innate partnership of HLA-B and KIR3DL1 subtypes against HIV-1. Nature genetics 2007, 39(6):733-740.

64. Gardiner CM, Guethlein LA, Shilling HG, Pando M, Carr WH, Rajalingam R, Vilches C, Parham P: Different NK cell surface phenotypes defined by the DX9 antibody are due to KIR3DL1 gene polymorphism. Journal of immunology (Baltimore, Md: 1950) 2001, 166(5):2992-3001.

65. Robinson J, Halliwell JA, McWilliam H, Lopez R, Marsh SGE: IPD--the Immuno Polymorphism Database. Nucleic Acids Res 2013, 41(Database issue):D1234-D1240.

66. Cooper DN: Functional intronic polymorphisms: Buried treasure awaiting discovery within our genes. Hum Genomics 2010, 4(5):284-288.

67. Toscano C, Klein K, Blievernicht J, Schaeffeler E, Saussele T, Raimundo S, Eichelbaum M, Schwab M, Zanger UM: Impaired expression of CYP2D6 in intermediate metabolizers carrying the *41 allele caused by the intronic SNP 2988G >A: evidence for modulation of splicing events. Pharmacogenetics and genomics 2006, 16(10):755-766.

68. Thomas R, Thio CL, Apps R, Qi Y, Gao X, Marti D, Stein JL, Soderberg KA, Moody MA, Goedert JJ et al: A novel variant marking HLA-DP expression levels predicts recovery from hepatitis B virus infection. Journal of virology 2012, 86(12):6979-6985.

69. Hodgkinson A, Eyre-Walker A: Variation in the mutation rate across mammalian genomes. Nature Reviews Genetics 2011, 12(11):756-766.

70. Hwang DG, Green P: Bayesian Markov chain Monte Carlo sequence analysis reveals varying neutral substitution patterns in mammalian evolution. Proc Natl Acad Sci U S A 2004, 101(39):13994-14001.

71. Nachman MW, Crowell SL: Estimate of the mutation rate per nucleotide in humans. Genetics 2000, 156(1):297304.

72. Nachman MW: Single nucleotide polymorphisms and recombination rate in humans. Trends in genetics: TIG 2001, 17(9):481-485.

73. Lercher MJ, Hurst LD: Human SNP variability and mutation rate are higher in regions of high recombination. Trends in genetics : TIG 2002, 18(7):337-340.

74. Duret L, Arndt PF: The Impact of Recombination on Nucleotide Substitutions in the Human Genome. PLoS genetics 2008, 4(5):e1000071.

75. Tian D, Wang Q, Zhang P, Araki H, Yang S, Kreitman M, Nagylaki T, Hudson R, Bergelson J, Chen JQ: Singlenucleotide mutation rate increases close to insertions/deletions in eukaryotes. Nature 2008, 455(7209):105108.

76. Zhu L, Wang Q, Tang P, Araki H, Tian D: Genomewide association between insertions/deletions and the nucleotide diversity in bacteria. Molecular biology and evolution 2009, 26(10):2353-2361.

77. McDonald MJ, Wang W-C, Huang H-D, Leu J-Y: Clusters of Nucleotide Substitutions and Insertion/Deletion Mutations Are Associated with Repeat Sequences. PLOS Biology 2011, 9(6):e1000622.

78. Maxwell LD, Williams F, Gilmore P, Meenagh A, Middleton D: Investigation of killer cell immunoglobulin-like receptor gene diversity: II. KIR2DS4. Human immunology 2004, 65(6):613-621.

79. Bruijnesteijn J, van der Wiel MKH, de Groot N, Otting N, de Vos-Rouweler AJM, Lardy NM, de Groot NG, Bontrop RE: Extensive Alternative Splicing of KIR Transcripts. Frontiers in immunology 2018, 9:2846.

80. Vilches C, Rajalingam R, Uhrberg M, Gardiner CM, Young NT, Parham P: KIR2DL5, a Novel Killer-Cell Receptor with a DO-D2 Configuration of Ig-Like Domains. The Journal of Immunology 2000, 164(11):5797.

81. Dohring C, Samaridis J, Colonna M: Alternatively spliced forms of human killer inhibitory receptors. Immunogenetics 1996, 44(3):227-230. 
82. Wilson MJ, Torkar M, Trowsdale J: Genomic organization of a human killer cell inhibitory receptor gene. Tissue antigens $1997,49(6): 574-579$.

83. Blokhuis JH, Doxiadis GG, Bontrop RE: A splice site mutation converts an inhibitory killer cell Ig-like receptor into an activating one. Molecular immunology 2009, 46(4):640-648.

84. Lee Y, Rio DC: Mechanisms and Regulation of Alternative Pre-mRNA Splicing. Annual Review of Biochemistry 2015, 84(1):291-323.

85. Goodridge JP, Lathbury LJ, Steiner NK, Shulse CN, Pullikotil P, Seidah NG, Hurley CK, Christiansen FT, Witt CS: Three common alleles of KIR2DL4 (CD158d) encode constitutively expressed, inducible and secreted receptors in NK cells. European journal of immunology 2007, 37(1):199-211.

86. Rajalingam R, Parham P, Abi-Rached L: Domain shuffling has been the main mechanism forming new hominoid killer cell Ig-like receptors. Journal of immunology (Baltimore, Md : 1950) 2004, 172(1):356-369.

87. Valiante NM, Uhrberg M, Shilling HG, Lienert-Weidenbach K, Arnett KL, D’Andrea A, Phillips JH, Lanier LL, Parham P: Functionally and structurally distinct NK cell receptor repertoires in the peripheral blood of two human donors. Immunity 1997, 7(6):739-751.

88. Andersson S, Malmberg JA, Malmberg KJ: Tolerant and diverse natural killer cell repertoires in the absence of selection. Experimental cell research 2010, 316(8):1309-1315.

89. Santourlidis S, Trompeter HI, Weinhold S, Eisermann B, Meyer KL, Wernet P, Uhrberg M: Crucial role of DNA methylation in determination of clonally distributed killer cell Ig-like receptor expression patterns in NK cells. Journal of immunology (Baltimore, Md : 1950) 2002, 169(8):4253-4261.

90. Chan H-W, Kurago ZB, Stewart CA, Wilson MJ, Martin MP, Mace BE, Carrington M, Trowsdale J, Lutz CT: DNA methylation maintains allele-specific KIR gene expression in human natural killer cells. The Journal of experimental medicine 2003, 197(2):245-255.

91. Stewart CA, Van Bergen J, Trowsdale J: Different and divergent regulation of the KIR2DL4 and KIR3DL1 promoters. Journal of immunology (Baltimore, Md : 1950) 2003, 170(12):6073-6081.

92. Trompeter HI, Gomez-Lozano N, Santourlidis S, Eisermann B, Wernet P, Vilches C, Uhrberg M: Three structurally and functionally divergent kinds of promoters regulate expression of clonally distributed killer cell Ig-like receptors (KIR), of KIR2DL4, and of KIR3DL3. Journal of immunology (Baltimore, Md : 1950) 2005, 174(7):41354143.

93. Li H, Pascal V, Martin MP, Carrington M, Anderson SK: Genetic Control of Variegated KIR Gene Expression: Polymorphisms of the Bi-Directional KIR3DL1 Promoter Are Associated with Distinct Frequencies of Gene Expression. PLoS genetics 2008, 4(11):e1000254.

94. Davies GE, Locke SM, Wright PW, Li H, Hanson RJ, Miller JS, Anderson SK: Identification of bidirectional promoters in the human KIR genes. Genes and immunity 2007, 8(3):245-253.

95. Li H, Wright PW, McCullen M, Anderson SK: Characterization of KIR intermediate promoters reveals four promoter types associated with distinct expression patterns of KIR subtypes. Genes and immunity 2016, 17(1):66-74.

96. Parham P: Immunogenetics of killer-cell immunoglobulin-like receptors. Tissue antigens 2003, 62(3):194-200.

97. Gomez-Lozano N, Trompeter HI, de Pablo R, Estefania E, Uhrberg M, Vilches C: Epigenetic silencing of potentially functional KIR2DL5 alleles: Implications for the acquisition of KIR repertoires by NK cells. European journal of immunology 2007, 37(7):1954-1965.

98. Cisneros E, Moraru M, Gómez-Lozano N, López-Botet M, Vilches C: KIR2DL5: An Orphan Inhibitory Receptor Displaying Complex Patterns of Polymorphism and Expression. Frontiers in immunology 2012, 3:289-289.

99. Vilches C, Gardiner CM, Parham P: Gene Structure and Promoter Variation of Expressed and Nonexpressed Variants of the \&It;em\&gt;KIR2DL5\&It;/em\&gt; Gene. The Journal of Immunology 2000, 165(11):6416.

100. Gomez-Lozano N, Estefania E, Williams F, Halfpenny I, Middleton D, Solis R, Vilches C: The silent KIR3DP1 gene (CD158C) is transcribed and might encode a secreted receptor in a minority of humans, in whom the KIR3DP1, KIR2DL4 and KIR3DL1/KIR3DS1 genes are duplicated. European journal of immunology 2005, 35(1):16-24.

101. van Bergen J, Stewart CA, van den Elsen PJ, Trowsdale J: Structural and functional differences between the promoters of independently expressed killer cell Ig-like receptors. European journal of immunology 2005, 35(7):2191-2199. 
102. Marquardt N, Scharenberg M, Mold JE, Hård J, Kekäläinen E, Buggert M, Nguyen S, Wilson JN, Al-Ameri M, Ljunggren $\mathrm{H}-\mathrm{G}$ et al: High-dimensional analysis reveals a distinct population of adaptive-like tissue-resident NK cells in human lung. bioRxiv 2019:2019.2012.2020.883785.

103. Marquardt N, Beziat V, Nystrom S, Hengst J, Ivarsson MA, Kekalainen E, Johansson H, Mjosberg J, Westgren $\mathrm{M}$, Lankisch TO et al: Cutting edge: identification and characterization of human intrahepatic CD49a+ NK cells. Journal of immunology (Baltimore, Md: 1950) 2015, 194(6):2467-2471.

104. Ivarsson MA, Stiglund N, Marquardt N, Westgren M, Gidlof S, Bjorkstrom NK: Composition and dynamics of the uterine NK cell KIR repertoire in menstrual blood. Mucosal immunology 2017, 10(2):322-331.

105. Björkström NK, Béziat V, Cichocki F, Liu LL, Levine J, Larsson S, Koup RA, Anderson SK, Ljunggren H-G, Malmberg K-J: CD8 T cells express randomly selected KIRs with distinct specificities compared with NK cells. Blood 2012, 120(17):3455-3465.

106. Mingari MC, Moretta A, Moretta L: Regulation of KIR expression in human T cells: a safety mechanism that may impair protective T-cell responses. Immunology today 1998, 19(4):153-157.

107. Huard B, Karlsson L: KIR expression on self-reactive CD8+ T cells is controlled by T-cell receptor engagement. Nature 2000, 403(6767):325-328.

108. Liu Y, Kuick R, Hanash S, Richardson B: DNA methylation inhibition increases T cell KIR expression through effects on both promoter methylation and transcription factors. Clin Immunol 2009, 130(2):213-224.

109. Chan H-W, Kurago ZB, Stewart CA, Wilson MJ, Martin MP, Mace BE, Carrington M, Trowsdale J, Lutz CT: DNA Methylation Maintains Allele-specific KIR Gene Expression in Human Natural Killer Cells. Journal of Experimental Medicine 2003, 197(2):245-255.

110. Lam TH, Shen M, Chia JM, Chan SH, Ren EC: Population-specific recombination sites within the human MHC region. Heredity 2013, 111(2):131-138.

111. Miretti MM, Walsh EC, Ke X, Delgado M, Griffiths M, Hunt S, Morrison J, Whittaker P, Lander ES, Cardon LR et al: A High-Resolution Linkage-Disequilibrium Map of the Human Major Histocompatibility Complex and First Generation of Tag Single-Nucleotide Polymorphisms. The American Journal of Human Genetics 2005, 76(4):634-646.

112. Voorter CE, Gerritsen KE, Groeneweg M, Wieten L, Tilanus MG: The role of gene polymorphism in HLA class I splicing. International journal of immunogenetics 2016, 43(2):65-78.

113. Krangel MS: Secretion of HLA-A and-B antigens via an alternative RNA splicing pathway. Journal of Experimental Medicine 1986, 163(5):1173-1190.

114. Dunn PP, Hammond L, Coates E, Street J, Griner L, Darke C: A "silent" nucleotide substitution in exon 4 is responsible for the "alternative expression" of HLA-A*01:01:38L through aberrant splicing. Human immunology 2011, 72(9):717-722.

115. Dai Z-X, Zhang G-H, Zhang X-H, Xia H-J, Li S-Y, Zheng Y-T: The $\beta 2$-Microglobulin-Free Heterodimerization of Rhesus Monkey MHC Class I A with Its Normally Spliced Variant Reduces the Ubiquitin-Dependent Degradation of MHC Class I A. The Journal of Immunology 2012:1100665.

116. Dai ZX, Zhang GH, Zhang XH, Zheng YT: Identification and characterization of a novel splice variant of rhesus macaque MHC IA. Molecular immunology 2013, 53(3):206-213.

117. Ishitani A, Geraghty DE: Alternative splicing of HLA-G transcripts yields proteins with primary structures resembling both class I and class II antigens. Proceedings of the National Academy of Sciences 1992, 89(9):3947.

118. Rouas-Freiss N, Bruel S, Menier C, Marcou C, Moreau P, Carosella ED: Switch of HLA-G alternative splicing in a melanoma cell line causes loss of HLA-G1 expression and sensitivity to NK lysis. International Journal of Cancer 2005, 117(1):114-122.

119. Kirszenbaum M, Moreau P, Gluckman E, Dausset J, Carosella E: An alternatively spliced form of HLA-G mRNA in human trophoblasts and evidence for the presence of HLA-G transcript in adult lymphocytes. Proceedings of the National Academy of Sciences 1994, 91(10):4209.

120. Rizzo R, Bortolotti D, Bolzani S, Fainardi E: HLA-G Molecules in Autoimmune Diseases and Infections. Frontiers in immunology 2014, 5:592-592.

121. Fleming KA, McMichael A, Morton JA, Woods J, McGee JO: Distribution of HLA class 1 antigens in normal human tissue and in mammary cancer. Journal of clinical pathology 1981, 34(7):779-784. 
122. Wei X, Orr HT: HLA Class I. In: Encyclopedia of Immunology (Second Edition). Edited by Delves PJ. Oxford: Elsevier; 1998: 1108-1111.

123. Dellgren C, Nehlin JO, Barington T: Cell surface expression level variation between two common Human Leukocyte Antigen alleles, HLA-A2 and HLA-B8, is dependent on the structure of the $C$ terminal part of the alpha 2 and the alpha 3 domains. PLoS One 2015, 10(8):e0135385.

124. Apps R, Meng Z, Del Prete GQ, Lifson JD, Zhou M, Carrington M: Relative expression levels of the HLA class-I proteins in normal and HIV-infected cells. Journal of immunology (Baltimore, Md: 1950) 2015, 194(8):35943600 .

125. Apps R, Qi Y, Carlson JM, Chen H, Gao X, Thomas R, Yuki Y, Del Prete GQ, Goulder P, Brumme ZL et al: Influence of HLA-C Expression Level on HIV Control. Science 2013, 340(6128):87.

126. Otting N, Heijmans CM, Noort RC, de Groot NG, Doxiadis GG, van Rood JJ, Watkins DI, Bontrop RE: Unparalleled complexity of the MHC class I region in rhesus macaques. Proc Natl Acad Sci U S A 2005, 102(5):1626-1631.

127. Otting N, Heijmans CM, van der Wiel M, de Groot NG, Doxiadis GG, Bontrop RE: A snapshot of the Mamu-B genes and their allelic repertoire in rhesus macaques of Chinese origin. Immunogenetics 2008, 60(9):507-514

128. He Y, Tian Z: NK cell education via nonclassical MHC and non-MHC ligands. Cellular \& molecular immunology 2017, 14(4):321-330.

129. He Y, Peng H, Sun R, Wei H, Ljunggren H-G, Yokoyama WM, Tian Z: Contribution of inhibitory receptor TIGIT to NK cell education. Journal of Autoimmunity 2017, 81:1-12.

130. Horowitz A, Djaoud Z, Nemat-Gorgani N, Blokhuis J, Hilton HG, Béziat V, Malmberg KJ, Norman PJ, Guethlein LA, Parham P: Class I HLA haplotypes form two schools that educate NK cells in different ways. Science immunology 2016, 1(3).

131. Anfossi N, André P, Guia S, Falk CS, Roetynck S, Stewart CA, Breso V, Frassati C, Reviron D, Middleton D et al: Human NK Cell Education by Inhibitory Receptors for MHC Class I. Immunity 2006, 25(2):331-342.

132. Borrego F, Ulbrecht M, Weiss EH, Coligan JE, Brooks AG: Recognition of human histocompatibility leukocyte antigen (HLA)-E complexed with HLA class I signal sequence-derived peptides by CD94/NKG2 confers protection from natural killer cell-mediated lysis. The Journal of experimental medicine 1998, 187(5):813-818.

133. Braud VM, Allan DS, O'Callaghan CA, Soderstrom K, D'Andrea A, Ogg GS, Lazetic S, Young NT, Bell JI, Phillips JH et al: HLA-E binds to natural killer cell receptors CD94/NKG2A, B and C. Nature 1998, 391(6669):795-799.

134. Lee N, Llano M, Carretero M, Ishitani A, Navarro F, López-Botet M, Geraghty DE: HLA-E is a major ligand for the natural killer inhibitory receptor CD94/NKG2A. Proc Natl Acad Sci U S A 1998, 95(9):5199-5204.

135. Fauriat C, Ivarsson MA, Ljunggren HG, Malmberg KJ, Michaëlsson J: Education of human natural killer cells by activating killer cell immunoglobulin-like receptors. Blood 2010, 115(6):1166-1174.

136. Lee N, Goodlett DR, Ishitani A, Marquardt H, Geraghty DE: HLA-E surface expression depends on binding of TAP-dependent peptides derived from certain HLA class I signal sequences. Journal of immunology (Baltimore, Md : 1950) 1998, 160(10):4951-4960.

137. Malnati MS, Peruzzi M, Parker KC, Biddison WE, Ciccone E, Moretta A, Long EO: Peptide specificity in the recognition of MHC class I by natural killer cell clones. Science 1995, 267(5200):1016-1018.

138. Rajagopalan S, Long EO: The direct binding of a p58 killer cell inhibitory receptor to human histocompatibility leukocyte antigen (HLA)-CW4 exhibits peptide selectivity. The Journal of experimental medicine 1997, 185(8):1523-1528.

139. Hansasuta P, Dong T, Thananchai H, Weekes M, Willberg C, Aldemir H, Rowland-Jones S, Braud VM: Recognition of HLA-A3 and HLA-A11 by KIR3DL2 is peptide-specific. European journal of immunology 2004, 34(6):16731679.

140. Fadda L, Borhis G, Ahmed P, Cheent K, Pageon SV, Cazaly A, Stathopoulos S, Middleton D, Mulder A, Claas FHJ et al: Peptide antagonism as a mechanism for NK cell activation. Proceedings of the National Academy of Sciences 2010, 107(22):10160.

141. Li Y, Mariuzza RA: Structural basis for recognition of cellular and viral ligands by NK cell receptors. Frontiers in immunology 2014, 5:123.

142. Carrillo-Bustamante P, de Boer RJ, Keşmir C: Specificity of inhibitory KIRs enables NK cells to detect changes in an altered peptide environment. Immunogenetics 2018, 70(2):87-97. 
143. Yu J, Heller G, Chewning J, Kim S, Yokoyama WM, Hsu KC: Hierarchy of the Human Natural Killer Cell Response Is Determined by Class and Quantity of Inhibitory Receptors for Self-HLA-B and HLA-C Ligands. The Journal of Immunology 2007, 179(9):5977.

144. Yawata M, Yawata N, Draghi M, Little A-M, Partheniou F, Parham P: Roles for HLA and KIR polymorphisms in natural killer cell repertoire selection and modulation of effector function. The Journal of experimental medicine 2006, 203(3):633-645.

145. Shilling HG, Young N, Guethlein LA, Cheng NW, Gardiner CM, Tyan D, Parham P: Genetic control of human NK cell repertoire. Journal of immunology (Baltimore, Md : 1950) 2002, 169(1):239-247.

146. Yu J, Heller G, Chewning J, Kim S, Yokoyama WM, Hsu KC: Hierarchy of the human natural killer cell response is determined by class and quantity of inhibitory receptors for self-HLA-B and HLA-C ligands. Journal of immunology (Baltimore, Md: 1950) 2007, 179(9):5977-5989.

147. Templer S, Sacks G: A blessing and a curse: is high NK cell activity good for health and bad for reproduction? Human fertility (Cambridge, England) 2016, 19(3):166-172.

148. Shakhar K, Rosenne E, Loewenthal R, Shakhar G, Carp H, Ben-Eliyahu S: High NK cell activity in recurrent miscarriage: what are we really measuring? Human reproduction (Oxford, England) 2006, 21(9):2421-2425.

149. Colucci F: The role of KIR and HLA interactions in pregnancy complications. Immunogenetics 2017, 69(8):557565.

150. Hiby SE, Regan L, Lo W, Farrell L, Carrington M, Moffett A: Association of maternal killer-cell immunoglobulinlike receptors and parental HLA-C genotypes with recurrent miscarriage. Human reproduction (Oxford, England) 2008, 23(4):972-976.

151. Tripathy SK, Keyel PA, Yang L, Pingel JT, Cheng TP, Schneeberger A, Yokoyama WM: Continuous engagement of a self-specific activation receptor induces NK cell tolerance. The Journal of experimental medicine 2008, 205(8):1829-1841.

152. Liao NS, Bix M, Zijlstra M, Jaenisch R, Raulet D: MHC class I deficiency: susceptibility to natural killer (NK) cells and impaired NK activity. Science 1991, 253(5016):199-202.

153. Hoglund P, Ohlen C, Carbone E, Franksson L, Ljunggren HG, Latour A, Koller B, Karre K: Recognition of beta 2-microglobulin-negative (beta $2 \mathrm{~m}$-) T-cell blasts by natural killer cells from normal but not from beta $2 \mathrm{~m}$ - mice: nonresponsiveness controlled by beta $2 \mathrm{~m}$ - bone marrow in chimeric mice. Proc Natl Acad Sci U S A 1991, 88(22):10332-10336.

154. Norman PJ, Hollenbach JA, Nemat-Gorgani N, Marin WM, Norberg SJ, Ashouri E, Jayaraman J, Wroblewski EE, Trowsdale J, Rajalingam R et al: Defining KIR and HLA Class I Genotypes at Highest Resolution via HighThroughput Sequencing. American journal of human genetics 2016, 99(2):375-391.

155. Doherty PC, Zinkernagel RM: Enhanced immunological surveillance in mice heterozygous at the $\mathrm{H}-2$ gene complex. Nature 1975, 256(5512):50-52.

156. McClelland EE, Penn DJ, Potts WK: Major histocompatibility complex heterozygote superiority during coinfection. Infect Immun 2003, 71(4):2079-2086.

157. Penn DJ, Damjanovich K, Potts WK: MHC heterozygosity confers a selective advantage against multiple-strain infections. Proc Natl Acad Sci U S A 2002, 99(17):11260-11264.

158. Tu MM, Mahmoud AB, Makrigiannis AP: Licensed and Unlicensed NK Cells: Differential Roles in Cancer and Viral Control. Frontiers in immunology 2016, 7(166).

159. Tarek N, Le Luduec JB, Gallagher MM, Zheng J, Venstrom JM, Chamberlain E, Modak S, Heller G, Dupont B, Cheung NK et al: Unlicensed NK cells target neuroblastoma following anti-GD2 antibody treatment. The Journal of clinical investigation 2012, 122(9):3260-3270.

160. Orr MT, Murphy WJ, Lanier LL: 'Unlicensed' natural killer cells dominate the response to cytomegalovirus infection. Nature immunology 2010, 11(4):321-327.

161. Maccari G, Robinson J, Ballingall K, Guethlein LA, Grimholt U, Kaufman J, Ho CS, de Groot NG, Flicek P, Bontrop RE et al: IPD-MHC 2.0: an improved inter-species database for the study of the major histocompatibility complex. Nucleic Acids Res 2017, 45(D1):D860-d864. 



\section{General discussion and future prospects}

Jesse Bruijnesteijn 


\section{General discussion and future prospects}

Non-human primates are important models for many human diseases that involve different components of the immune system. For that reason, a detailed characterization of these components, and in particular the similarity to its equivalent in humans, is a necessity. During the course of this thesis, a comparative immunogenetic approach was chosen to unravel the complexity of a set of important immune receptors that are expressed by natural killer (NK) cells. NK cells are cytotoxic lymphocytes, which provide a rapid and non-specific immune response analogous to that of cytotoxic T cells (CTLS) [1]. The adaptive response of CTLs is far more specific, as their activation and clonal expansion depend on rearranged antigen-specific receptors that are educated in the thymus. Expression of different classes of germline-encoded activating and inhibitory receptors tightly control the education and activity of NK cells. The killer cell immunoglobulin-like receptor (KIR) family is one of the major components that is involved in this regulation, and mainly acts through interactions with major histocompatibility complex (MHC) class I molecules. In contrast to the T cell receptors, the KIR receptors are not subjected to rearrangement and have an innate character. Although KIR may recognize peptides in context of $\mathrm{MHC}$, they primarily seem to interact with a limited set of polymorphic determinants on $\mathrm{MHC}$ class I molecules. The human KIR genes display a high degree of diversity, which is reflected by copy number variation (CNV), allelic polymorphism, and different haplotype configurations [2, 3]. This genetic diversification of KIR might be propelled by selective co-evolution with their $\mathrm{MHC}$ ligands, and both systems are in a continuous arms race with pathogens. For decades, the $M H C$ region was considered the most polymorphic and variable gene system in primates, but the KIR cluster seems to exceed this plasticity [4, 5]. The diverse compositions and allelic variety of $K I R$ genes at an individual and populational level is highly relevant in relation to disease susceptibility and protection, which advocates for a comprehensive characterization in humans [6-8].

The genetic KIR cluster is to some extent also characterized in several non-human primate species. We focused on the thorough characterization of KIR genes in rhesus and cynomolgus macaques, whereas others have defined the KIR cluster in chimpanzees, orangutans, and baboons [9-12]. Understanding the genetics and evolution of primate KIR provides a foundation to study their functional impact related to health and disease, and might also help to refine disease models. The NK cell biology of macaques and humans display great similarity, which is in sharp contrast to the largely diverged NK cells in rodents. Therefore, macaque species seem to be a powerful model to study NK cells and KIR receptor function in relation to health and disease. 


\section{High accuracy characterization of the human and rhesus macaque KIR genes}

In recent years, the KIR gene cluster in humans is extensively examined using different conventional sequencing approaches. Most of these studies resolved the genomic KIR content based on the presence or absence of particular gene segments by amplification with sequence-specific primers [13-15]. This approach may miss out on essential information on allelic polymorphism, recombinant genes, copy number variation (CNV), gene expression levels, and post-transcriptional modifications, such as alternative splicing. Less frequent was the characterization of complete human KIR haplotypes, but has been demonstrated using Fosmid library sequencing and targetenriched illumuna sequencing $[16,17]$. Contrasting to the mainly genomic strategy in humans, the KIR genes in macaques were initially characterized by Sanger sequencing of partial and full-length cDNA sequences that were amplified by sequence-specific primers $[18,19]$. Several macaque $K I R$ haplotype configurations were deduced by studying gene segregation in families, and revealed extensive expansions and contractions. Although these early studies were informative, the conventional characterization strategies often lack an allele level resolution, and might miss genes with low transcription levels. These shortcomings are mostly explained by a relatively low throughput and limited fragment length of the used techniques, such as Roche 454 and Sanger sequencing. The development of single-molecule real-time (SMRT) sequencing platforms, which are commercialized by Pacific Biosciences (PacBio) and Oxford Nanopore Technologies (ONT), overcome many of the conventional limitations.

The PacBio platform generates sequence information during real time synthesis of complement DNA using fluorescent nucleotides. This technique offers long read lengths and high throughput. The relatively high error rate compared to conventional sequencing methods is largely compensated by sequencing one strand multiple times, and subsequently allows one to generate a consensus sequence. The high accuracy and throughput of PacBio consensus sequencing allows complete definition of KIR transcriptomes in humans and rhesus macaques (Chapter $\mathbf{2}$ ). In this study, full-length $K I R$ transcripts were characterized, which resolved and confirmed novel alleles. Even more, fusion gene transcripts, which comprised head and tail segments from different $K I R$ genes, indicated recombination events in the human and rhesus macaque KIR cluster. Using a family-based study design, segregation analysis enabled the definition of KIR haplotype configurations. In humans, KIR haplotypes are largely conform a typical centromeric and telomeric region, with either a more inhibitory or activating gene content (group A or B haplotypes). More dynamic gene content variability is featured for rhesus macaque KIR haplotype configurations, as reflected by expansions, contractions, and the generation of substantial numbers of fusion genes. The comprehensive overview of human and rhesus macaque KIR transcriptomes demonstrates the power of the new characterization strategy, which is relatively fast, cost-efficient, and highly accurate [20]. 


\section{Unparalleled rapid expansion of $K I R$ genes in macaque species and their populations}

Characterization of the KIR gene cluster in different distantly related primate species, such as humans, chimpanzees, orangutans, and macaques, provided a comparative overview of its evolution [21]. Extensive tandem duplications, deletions, and recombination events shaped the KIR region in these primates, and seems to be largely propelled by co-evolution with their MHC class I ligands. The dynamics and plasticity of the KIR gene cluster might, however, be better reflected by comparing closely related primate species that share similar MHC class I systems. Using the PacBio full-length transcriptome strategy, we characterized the KIR gene content in rhesus and cynomolgus macaques, and their geographically distinct populations (Chapter $\mathbf{3}$ ). These two macaque species are relatively closely related, with a shared common ancestor that lived approximately 1-3 million years ago.

A profound overview of the KIR gene system was defined in both macaque species, which recorded 34 rhesus and 55 cynomolgus macaque KIR genes. Of these genes, 24 were considered orthologous, thereby reflecting the close common ancestry, whereas the considerable number of species-specific entities indicate a rapid evolution. In both species, the number of fusion gene entities indicated abundant chromosomal recombination events. This was substantiated by the highly dynamic KIR haplotype content that involved expansions and contractions. Several KIR genes were not only found specific for one species, but were also restricted to a particular macaque population. These population-specific KIR genes suggest an unprecedented rapid evolution of the genetic cluster. Even more, the macaque KIR genes also feature extensive allelic polymorphism, with 285 rhesus and 260 cynomolgus macaque allotypes defined in the studied cohorts. Only two KIR allotypes were recorded the be shared in both species, and even the different macaque populations only shared one or two allelic variants.

The unpararelled rapid evolution of the macaque $K I R$ region diversifies their genetic content at a species and populational level. This KIR diversity might modulate the outcome of preclinical studies using macaque models. A better understanding of the genetic profile of macaques, for instance based on their species- and populationspecific KIR gene content, might help to refine biomedical experiments. These advanced insights might better the translation of results in macaque models to humans. This might shed a light on the different KIR and MHC class I combinations that have been associated with disease susceptibility and protection, and eventually help to develop medicines and therapies for humans. 


\section{A wide variety of KIR isoforms: functional relevance or transcriptional noise?}

The full-length KIR transcriptomes derived from the PacBio studies not only defined constitutively spliced transcripts, but also resolved high numbers of different alternatively spliced variants. Some of these posttranscriptional modifications were previously documented [22-24], but a comprehensive overview of the KIR spliceosome was lacking. In a follow-up study, we used the PacBio protocol to examine the alternative splicing profiles in several human and rhesus macaque families (Chapter 4). In total, 18 and 29 distinct splice events were recorded in humans and rhesus macaques, respectively, and were confirmed in at least two individuals. These splice events, of which some were gene-specific, were facilitated by different splicing mechanisms, such as exon skipping and the use of alternative splice sites. At this stage, the structural and functional consequences of the different splice events is hard to predict. Several splice events introduce an early stop codon, and might thereby abandon receptor function. Such an event might be noise in the transcriptional process, but might also have biological relevance as they function as a rapid switch to modulate receptor expression levels. Human 9A KIR2DL4 alleles, for example, encode constitutively truncated receptors, and its expression is not detectable [25]. The use of an alternative splice site includes an intronic stretch to generate 9A KIR2DL4 transcripts that seem to encode complete receptor isoforms. In a transfection study, we demonstrated that these 9A KIR2DL4 isoforms were expressed in endosomes (manuscript in preparation). In contrast, the same splice event in the in-frame 10A KIR2DL4 allotypes results in undetectable isoforms, which suggests that alternative splicing might function as an expression modulator.

The selection and conservation of different alternative splice sites suggests a functional role for particular isoforms. A highly conserved splice event in macaques, for instance, involves their only framework gene, KIR3DL20. Transcripts of KIR3DL20 encode KIR3D and KIR2D receptors, the latter of which are consistently generated by the exclusion of exon 4 from KIR3D transcripts. These alternatively spliced transcripts display sequence similarity to human KIR2DL5, and are therefore referred to as Mamuand Mafa-KIR2DL05. The function of KIR2DL05 isoforms in macaques, or its equivalent in humans, is not known. Nevertheless, alternative splicing seems to expand the framework KIR repertoire in macaques, and might facilitate a form of convergent evolution to generate KIR2DL5-like receptors.

With a comprehensive overview of the KIR splicing profiles in humans and macaques, the main challenge remains to determine its biological relevance. A method to determine isoform quantities relative to the constitutively spliced transcripts might help in understanding isoform function. Most conventional sequencing and quantification methods, such as real-time $\mathrm{PPCR}$, are limited by short reads, and are insufficient to determine isoform quantities. The development of SMRT sequencing platforms might help in resolving isoform expression levels. This would provide insights into the distribution of KIR isoforms in different tissues or under certain conditions, and 
thereby indicate their functional relevance. Functional isoforms, and the regulation of its expression, has been documented in literature [26-28]. For instance, the induction of alternative splicing has been reported in NK cells for the tumor-recognition (NK-TR) gene, of which the constitutively spliced transcripts encode a truncated and nonfunctional receptor [26]. Upon activation of NK cells via IL-2 stimulation, the splicing pattern of NK cells changed, and functional NK-TR receptors were expressed. A similar mechanism might regulate the expression and function of KIR receptors and its isoforms. With the current technological sequencing developments and the profound knowledge on KIR splicing profiles, future experiments might aim at the understanding of isoform function.

\section{Genomic haplotype organization}

The characterization of the KIR genes at the transcription level fosters the knowledge on allelic variants, expression levels, and posttranscriptional modifications. Transcriptome studies lack, however, information on pseudogenes, the physical location of genes at the haplotype, and non-coding sequences, such as introns. Therefore, a genomic assembly of the complete multigenic region might provide additional insights. The complete genomic characterization of $K I R$ haplotypes is a challenging and time-consuming enterprise, as the region is large (100-300 kb), contains a dynamic gene content that share high sequence similarity, and harbors substantial repetitive elements. Indeed, the relatively standard human KIR haplotype configurations are well defined at the genomic DNA level using time-consuming Fosmid library and long-range PCR sequencing approaches [17, 29]. These completely sequenced haplotypes revealed a genomic architecture, in which centromeric and telomeric regions are occasionally shuffled (e.g., cA01-tA01, cB01-tB01, cA01-tB01). The haplotype reorganizations seem to be mediated by abundant transposable elements that are present in the non-coding regions. The genomic characterization of complete KIR haplotypes helps to understand how the haplotype diversity is generated, and what functional consequences might result from that.

Knowledge on the macaque KIR haplotypes is limited, and, so far, only two completely characterized short haplotypes are documented [30, 31]. Transcriptome and segregation studies, however, suggest an extensive gene content diversity, indicating expanded and contracted haplotypes. With the continuous and rapid evolution, and only a single framework gene, the macaque $K I R$ haplotypes are unlikely to follow relatively rigid organizations, as is documented for group A and B haplotypes in humans. The availability of more completely characterized macaque KIR haplotypes might improve the understanding and evolution of this highly dynamic gene cluster.

We developed a targeted enrichment protocol using Cas9 nuclease activity and Nanopore sequencing to characterize multigenic clusters, such as the KIR gene region (Chapter 5). Large and overlapping fragments are generated without amplification, which avoids errors by strand synthesis and retains modification information, like 
methylation profiles. The overlaps allow phasing of haplotypes, even at regions with high sequence similarity and in the absence of a reference genome. With this novel technological approach, we resolved six human and six rhesus macaque KIR haplotypes at an allele level resolution. This fast and relatively cheap characterization of complex immune regions might improve clinical protocols, such as the detailed matching of donor and acceptor compatibility in solid organ transplantation. In addition, more insights into the dynamic and complex macaque KIR haplotypes might help to understand the mechanism driving their extensive diversification. A comprehensive haplotype study may, for instance, reveal a linkage disequilibrium for different macaque $K I R$ gene combinations that is similar to some non-randomly associated human KIR genes, such as KIR2DL2-KIR2DS3-KIR2DL5B. The function of KIR alleles or genes that are consistently low expressed might be compensated by family members that display a genomic linkage. For example, in humans it is thought that the function of the poorly expressed KIR3DL1*004 allotype might be balanced by the presence of KIR3DL2 or/ and KIR2DL4 allotypes that display a strong linkage disequilibrium [32]. Therefore, defining linkage disequilibriums might help in understanding the concerted function of KIR receptors.

Overall, this strategy to define complex immune regions might provide a tool to improve clinical applications. In solid-organ transplantation, for instance, this tool might improve the resolution of KIR gene matching for the graft donor and acceptor, and thereby may contribute to an enhanced graft survival. The high resolution may also lift disease association studies from an allele to a haplotype/region level. In a functional perspective, the insights into the genomic KIR haplotypes and their epigenetic profiles might aid the understanding of receptor function. Furthermore, the characterization of complete KIR haplotypes helps to understand the evolution of highly dynamic gene clusters that are involved in modulating immune responses.

\section{A diverse group of immune receptors: impact on health and disease}

The primate KIR gene cluster might be one of the most plastic and dynamic regions of the genome, and is diversified by different molecular mechanisms (Chapter 6). The combination of these mechanisms, involving chromosomal recombination events, point mutations, alternative splicing, and variegated expression, generates an array of diversity that is reflected by haplotype contractions and expansions, recombinant genes, allelic polymorphism, structural distinct isoforms, and differential expression patterns. One might expect that this great diversity impacts NK cell education, activation, and cytotoxicity, and thereby modulate health and disease at an individual and populational level.

An important biological process in which NK cells are involved is pregnancy [33,34]. In the early stages of pregnancy, an semi-allogenic fetal trophoblast is implanted in the maternal uterus, which requires modulation of the local immune components. 
Specialized NK cells form the predominant population of lymphocytes during early pregnancy, and are suggested to be involved in trophoblast invasion and vascular remodelling. These NK cells display relatively low cytotoxic activity, and mainly produce cytokines and other regulatory factors that modulate the local immune environment. The variety of activating and inhibitory KIR receptors play an important role to balance the local NK cell activity. The expression of activating KIR, including KIR2DS1, KIR2DS4, and KIR2DS5, in combination with the presence of the HLA-C2 epitope, strongly enhance successful pregnancy [35-38]. The uterus-resident NK cells display increased expression of those activating receptors during different stages of pregnancy compared to periphial blood ( $\mathrm{pb}$ ) NK cells. The absence of activating KIR, their HLA-C2 ligands, or the presence of mainly inhibitory receptors on the uterus-resident NK cells might contribute to the opposite effect, and is associated with pre-eclampsia [39]. There are, however, more robust association studies required to determine the precise role of KIR (allotypes) and its HLA class I ligands in pregnancy, as contradicting results are documented [40].

As component of the immune system, NK cells also play a major role in disease, in which they are involved in the identification and lysis of infected cells, the surveillance of tumour cells, and the regulation of other immune components. The KIR gene content diversity and the extensive allelic polymorphism might impact susceptibility and protection to disease at an individual level, and increase survival at a populational level. These disease associations are often found in concert with the independently segregating MHC class I ligands. Several viral pathogeneses have been related to the genetic content of the KIR and MHC class I clusters. The diverse outcomes upon human immunodeficiency virus (HIV) infection, for example, is at least in part modulated by the polymorphisms of the KIR and HLA genes of the host [41, 42]. These HIV-related associations indicate susceptibility, with fast progression to acquired immunodeficiency syndrome (AIDS), or protection, with complete control of the infection without medicine. Other diseases associated with KIR gene diversity are, among others, tuberculosis, rheumatoid arthritis, and different types of cancer [43-45]. Disease associations studies are, however, only powerful when the involved genes are characterized in detail, the possible other interfering factors are considered, and the control group represents a substantial size. The difficulty to interpret these studies is demonstrated by potentially conflicting outcomes recorded in literature. A role for KIR2DS4, for instance, was associated with melanoma tumors through its interaction with non-classical HLA molecules [46], but this was not confirmed in a clinical study [47].

In the human population, the initial event or infection that proceeds disease is in many cases not known. The controlled study designs in animal models, however, allows one to study values for immunization or infection in an individual $(t=0)$, and follow the onset and course of disease, which facilitates more validated experiments. This might provide more robust associations of $K I R$ gene content and disease progress. For instance, infection of macaques with the simian immunodeficiency virus (SIV), which is similar to HIV-1 in humans, models the progression and symptoms of AIDS, and allowed 
to study the highly active antiretroviral therapy (HAART) $[48,49]$. These SIV studies demonstrated that the presence of particular KIR genes, including KIR3DLO2 and KIR3DSW08, were associated with protection to AIDS progression. A detailed characterization of the immune regions in macaques helps in selecting appropriate individuals for specific preclinical models, and thereby refine in vivo experiments.

\section{Functional implications of differential KIR gene expression}

The current studies on KIR receptors in humans, but also in other primate species, are mainly performed on periphial blood NK (pbNK) cells, where they account for approximately $10-15 \%$ of the circulating lymphocytes. The KIR transcriptomes and epigenetic profiles obtained from SMRT sequencing studies provide a proxy of the total $K I R$ repertoire present in an individual. A large proportion of NK cells, however, reside within peripheral and lymphoid organs, such as the uterus, liver, lungs, gut, and thymus [50-54]. These tissue-resident NK (trNK) cells may display distinct surface receptors that retain them within tissues and prevent egress into the periphery [55]. Even more, trNK cells might have different development pathways and execute specialized tissuerelated functions, as discussed above for uterus-resident NK cells in pregnancy [54, 55]. Knowledge on the KIR gene expression in these trNK cell populations is limited, but one might expect differential expression profiles compared to that observed in pbNK cells. This is for example demonstrated in lung- and liver-resident NK cells. In the liver, trNK cells exhibited an oligoclonal expression pattern of one, two, or three KIR receptors [50]. Samples from different liver donors did not indicate a clear dominance of a specific inhibitory KIR, but co-expression of two activating KIR was hardly observed. In the lungs, only the adaptive (or memory-like) NK cells that reside within the tissue displayed a distinct KIR expression profile contrasting to the profile in other lungresident or pbNK cell subsets [51]. This study, however, determined the expression of only four KIR genes, and might reflect an incomplete KIR phenotype [51]. The most extensively studied trNK cell population is the one that resides in the uterus [33, 36, 52]. The enhancement of trophoblast migration and vascular remodelling by the release of chemokines and cytokines from activated uterus-resident NK cells is a clear example of a tissue-specific NK cell function mediated by differential KIR expression.

The reason why the KIR expression profiles of trNK cells is poorly examined has practical and ethical reasons. Tissue samples from human organs are mainly harvested during surgery, such as lobectomy or liver resection for the removal of primary or metastatic tumours. These tissues are derived from patients that already have a compromised immune system, and might not resemble NK cells in a healthy tissue. With informed consent, tissues from healthy donor organs that were not used for transplantation can be harvested after death, but gene expression might be affected by a substantial period of time without organ perfusion [56]. Other options to study trNK cells include tissue cell line experiments, which might give indications for specific KIR gene expression profiles, but one has to take into account that this is always affected 
by in vitro circumstances. Animal models, however, might provide more insights into trNK cells and their specialized functions. As stated earlier, rodent animal models have a different NK cell biology, and tissue-specialized subpopulations might not translate to the human situation. Macaques might be the suitable species to examine the phenotype and function of trNK cells in a controlled and powerful model, which might broadly translate to human trNK cells. Even more, the many proven disease models in macaques allow association studies of phenotypical and functional NK cell variations, including differential KIR expression profiles, in regard to viral and bacterial infections. The profound insights on the macaque KIR genes gathered in the past decade might contribute to a better understanding of the specialized NK cell functions in tissues.

\section{Quantification of KIR gene transcripts and their isoforms}

The determination of differential KIR expression in distinct NK cell populations is only the first step to elucidate their biological role. Discrimination between high and low level expression of different KIR genes, and shifts in these expression levels, would provide additional insights. Fluctuations in expression level might be triggered by external factors, such as cell activation through cytokines, the presence of ligands, or pathogenic or tumorigenic conditions. Current quantification of $K I R$ transcripts rely on short fragment techniques, such as real-time qPCR and RNAseq in combination with Illumina sequencing $[51,57,58]$. These methods are able to discriminate the expression level of most human KIR genes. Differential KIR expression was, for example, demonstrated in NK/T-cell lymphoma's, in which KIR2DL4 was overexpressed compared to healthy conditions, whereas all other KIR were downregulated [58]. Short-read sequencing techniques are, however, irrespective to allelic variation, thereby omitting variable expression levels of KIR allotypes. Differential expression has been demonstrated for multiple KIR3DL1 allotypes, ranging from high, low, or no surface expression for particular allotypes. High-resolution quantification of KIR gene expression is challenging due to their extensive polymorphisms, copy number variation, and gene similarity. In macaques, the current short-read methods are insufficient to quantify the highly expanded KIR repertoire at all, considering the abundant fusion genes and gene similarities.

Direct sequencing of full-length RNA and cDNA transcripts using SMRT sequencing platforms enable quantitative transcriptome studies. A high coverage of transcripts is, however, required, to overcome the relatively low accuracy of these techniques. The thorough characterization of human and macaque KIR at the transcription level (Chapters $\mathbf{2}$ and $\mathbf{3}$ ) might contribute to the implementation of transcript quantification studies. In future experiments, the technological breakthroughs of SMRT sequencing platforms should be explored to gather more insights on the differential KIR expression patterns. This would help in defining the (specialized) functions of the distinct KIR receptors, which so far remained largely elusive. 


\section{The evolution of primate KIR genes}

This thesis, together with other characterization studies, provides a comprehensive overview of the extensive diversity and variation of the primate KIR genes. The KIR diversification might be largely driven by co-evolution with MHC class I genes, and thereby indirectly by the arms race with evolving pathogens. This co-evolution is substantiated by a balanced expansion of MHC and KIR in different primate species, with more expanded repertoires in macaques compared to hominoids. The plasticity of the KIR genes, however, exceeds that observed for the MHC class I genes, with more extensive allelic polymorphism, chromosomal recombination events, CNV, variegated expression, and alternative splicing. The wide variety of KIR receptors and isoforms, generated by a continuous evolutionary effort, regulate the NK cell responsiveness, which in turn modulates the elimination of infected or tumorigenic cells, the regulation of (adaptive) immune cells, and the trophoblast migration during different stages of pregnancy. The genomic and transcriptomic characterization of the primate KIR gene system is now largely elucidated, which paves the way to further explorer the function of KIR in different biological processes. 


\section{References}

1. Vivier E, Raulet DH, Moretta A, Caligiuri MA, Zitvogel L, Lanier LL, Yokoyama WM, Ugolini S: Innate or adaptive immunity? The example of natural killer cells. Science 2011, 331(6013):44-49.

2. Uhrberg M, Valiante NM, Shum BP, Shilling HG, Lienert-Weidenbach K, Corliss B, Tyan D, Lanier LL, Parham P: Human diversity in killer cell inhibitory receptor genes. Immunity 1997, 7(6):753-763.

3. Middleton D, Gonzelez F: The extensive polymorphism of KIR genes. Immunology 2010, 129(1):8-19.

4. Hedrick PW: Evolutionary Genetics of the Major Histocompatibility Complex. The American Naturalist 1994, 143(6):945-964.

5. Sommer S: The importance of immune gene variability ( $\mathrm{MHC}$ ) in evolutionary ecology and conservation. Frontiers in Zoology 2005, 2(1):16.

6. Khakoo SI, Thio CL, Martin MP, Brooks CR, Gao X, Astemborski J, Cheng J, Goedert JJ, Vlahov D, Hilgartner $\mathrm{M}$ et al: HLA and NK cell inhibitory receptor genes in resolving hepatitis C virus infection. Science 2004, 305(5685):872-874.

7. Qi Y, Martin MP, Gao X, Jacobson L, Goedert JJ, Buchbinder S, Kirk GD, O’Brien SJ, Trowsdale J, Carrington M: KIR/HLA pleiotropism: protection against both HIV and opportunistic infections. PLoS Pathog 2006, 2(8):e79.

8. Kulkarni S, Martin MP, Carrington M: The Yin and Yang of HLA and KIR in human disease. Seminars in Immunology 2008, 20(6):343-352.

9. Rajalingam R, Hong M, Adams EJ, Shum BP, Guethlein LA, Parham P: Short KIR Haplotypes in Pygmy Chimpanzee (Bonobo) Resemble the Conserved Framework of Diverse Human KIR Haplotypes. Journal of Experimental Medicine 2001, 193(1):135-146.

10. Abi-Rached L, Moesta AK, Rajalingam R, Guethlein LA, Parham P: Human-specific evolution and adaptation led to major qualitative differences in the variable receptors of human and chimpanzee natural killer cells. PLoS Genet 2010, 6(11):e1001192.

11. Wroblewski EE, Parham P, Guethlein LA: Two to Tango: Co-evolution of Hominid Natural Killer Cell Receptors and MHC. Frontiers in Immunology 2019, 10(177).

12. Guethlein LA, Norman PJ, Heijmans CM, de Groot NG, Hilton HG, Babrzadeh F, Abi-Rached L, Bontrop RE, Parham P: Two Orangutan Species Have Evolved Different KIR Alleles and Haplotypes. J Immunol 2017, 198(8):3157-3169.

13. Vilches C, Castaño J, Gómez-Lozano N, Estefanía E: Facilitation of KIR genotyping by a PCR-SSP method that amplifies short DNA fragments. Tissue Antigens 2007, 70(5):415-422.

14. Hong HA, Loubser AS, de Assis Rosa D, Naranbhai V, Carr W, Paximadis M, Lewis DA, Tiemessen CT, Gray CM: Killer-cell immunoglobulin-like receptor genotyping and HLA killer-cell immunoglobulin-like receptor-ligand identification by real-time polymerase chain reaction. Tissue Antigens 2011, 78(3):185-194.

15. Kulkarni S, Martin MP, Carrington M: KIR genotyping by multiplex PCR-SSP. Methods Mol Biol 2010, 612:365375.

16. Norman PJ, Hollenbach JA, Nemat-Gorgani N, Marin WM, Norberg SJ, Ashouri E, Jayaraman J, Wroblewski EE, Trowsdale J, Rajalingam R et al: Defining KIR and HLA Class I Genotypes at Highest Resolution via HighThroughput Sequencing. Am J Hum Genet 2016, 99(2):375-391.

17. Roe D, Vierra-Green C, Pyo CW, Eng K, Hall R, Kuang R, Spellman S, Ranade S, Geraghty DE, Maiers M: Revealing complete complex KIR haplotypes phased by long-read sequencing technology. Genes \& Immunity 2017, 18(3):127-134.

18. Blokhuis JH, van der Wiel MK, Doxiadis GGM, Bontrop RE: The mosaic of KIR haplotypes in rhesus macaques. Immunogenetics 2010, 62(5):295-306.

19. Bimber BN, Moreland AJ, Wiseman RW, Hughes AL, O'Connor DH: Complete characterization of killer Ig-like receptor (KIR) haplotypes in Mauritian cynomolgus macaques: novel insights into nonhuman primate KIR gene content and organization. J Immunol 2008, 181(9):6301-6308.

20. Bruijnesteijn J, van der Wiel MKH, Swelsen WTN, Otting N, de Vos-Rouweler AJM, Elferink D, Doxiadis GG, Claas FHJ, Lardy NM, de Groot NG et al: Human and Rhesus Macaque KIR Haplotypes Defined by Their Transcriptomes. J Immunol 2018, 200(5):1692-1701. 
21. Guethlein LA, Older Aguilar AM, Abi-Rached L, Parham P: Evolution of Killer Cell Ig-Like Receptor KIR Genes: Definition of an Orangutan KIR Haplotype Reveals Expansion of Lineage III KIR Associated with the Emergence of MHC-C. The Journal of Immunology 2007, 179(1):491.

22. Blokhuis JH, Doxiadis GG, Bontrop RE: A splice site mutation converts an inhibitory killer cell Ig-like receptor into an activating one. Mol Immunol 2009, 46(4):640-648.

23. Prall TM, Graham ME, Karl JA, Wiseman RW, Ericsen AJ, Raveendran M, Alan Harris R, Muzny DM, Gibbs RA, Rogers J et al: Improved full-length killer cell immunoglobulin-like receptor transcript discovery in Mauritian cynomolgus macaques. Immunogenetics 2017, 69(5):325-339.

24. Bimber BN, Evans DT: The killer-cell immunoglobulin-like receptors of macaques. Immunol Rev 2015, 267(1):246-258.

25. Bruijnesteijn J, van der Wiel MKH, de Groot N, Otting N, de Vos-Rouweler AJM, Lardy NM, de Groot NG, Bontrop RE: Extensive Alternative Splicing of KIR Transcripts. Front Immunol 2018, 9:2846.

26. Rinfret A, Anderson SK: IL-2 regulates the expression of the NK-TR gene via an alternate RNA splicing mechanism. Molecular Immunology 1993, 30(14):1307-1313.

27. Toei M, Saum R, Forgac M: Regulation and isoform function of the V-ATPases. Biochemistry 2010, 49(23):47154723.

28. Bhuiyan SA, Ly S, Phan M, Huntington B, Hogan E, Liu CC, Liu J, Pavlidis P: Systematic evaluation of isoform function in literature reports of alternative splicing. BMC Genomics 2018, 19(1):637.

29. Pyo C-W, Wang R, Vu Q, Cereb N, Yang SY, Duh F-M, Wolinsky S, Martin MP, Carrington M, Geraghty DE: Recombinant structures expand and contract inter and intragenic diversification at the KIR locus. BMC Genomics 2013, 14(1):89.

30. Graves T, Eichler,E.E. and Wilson,R.K.: Macaca mulatta isolate AG07107 chromosome 19, whole genome shotgun sequence. 2019.

31. Sambrook JG, Bashirova A, Palmer S, Sims S, Trowsdale J, Abi-Rached L, Parham P, Carrington M, Beck S: Single haplotype analysis demonstrates rapid evolution of the killer immunoglobulin-like receptor (KIR) loci in primates. Genome research 2005, 15(1):25-35.

32. Gourraud PA, Meenagh A, Cambon-Thomsen A, Middleton D: Linkage disequilibrium organization of the human KIR superlocus: implications for KIR data analyses. Immunogenetics 2010, 62(11-12):729-740.

33. Moffett-King A: Natural killer cells and pregnancy. Nature Reviews Immunology 2002, 2(9):656-663.

34. Faas MM, de Vos P: Uterine NK cells and macrophages in pregnancy. Placenta 2017, 56:44-52.

35. Hiby SE, Apps R, Sharkey AM, Farrell LE, Gardner L, Mulder A, Claas FH, Walker JJ, Redman CW, Morgan L et al: Maternal activating KIRs protect against human reproductive failure mediated by fetal HLA-C2. J Clin Invest 2010, 120(11):4102-4110.

36. Kennedy PR, Chazara O, Gardner L, Ivarsson MA, Farrell LE, Xiong S, Hiby SE, Colucci F, Sharkey AM, Moffett A: Activating KIR2DS4 Is Expressed by Uterine NK Cells and Contributes to Successful Pregnancy. J Immunol 2016, 197(11):4292-4300.

37. Hiby SE, Walker JJ, O'Shaughnessy K M, Redman CW, Carrington M, Trowsdale J, Moffett A: Combinations of maternal KIR and fetal HLA-C genes influence the risk of preeclampsia and reproductive success. J Exp Med 2004, 200(8):957-965.

38. Nakimuli A, Chazara O, Hiby SE, Farrell L, Tukwasibwe S, Jayaraman J, Traherne JA, Trowsdale J, Colucci F, Lougee E et al: A \&lt;em\&gt;KIR B\&lt;/em\&gt; centromeric region present in Africans but not Europeans protects pregnant women from pre-eclampsia. Proceedings of the National Academy of Sciences 2015, 112(3):845.

39. Hiby SE, Regan L, Lo W, Farrell L, Carrington M, Moffett A: Association of maternal killer-cell immunoglobulinlike receptors and parental HLA-C genotypes with recurrent miscarriage. Hum Reprod 2008, 23(4):972-976.

40. Colucci F: The role of KIR and HLA interactions in pregnancy complications. Immunogenetics 2017, 69(8):557565.

41. Bashirova AA, Thomas R, Carrington M: HLA/KIR restraint of HIV: surviving the fittest. Annu Rev Immuno/ 2011, 29:295-317. 
42. Alter G, Martin MP, Teigen N, Carr WH, Suscovich TJ, Schneidewind A, Streeck H, Waring M, Meier A, Brander C et al: Differential natural killer cell-mediated inhibition of HIV-1 replication based on distinct KIR/HLA subtypes. Journal of Experimental Medicine 2007, 204(12):3027-3036.

43. Pydi SS, Sunder SR, Venkatasubramanian S, Kovvali S, Jonnalagada S, Valluri VL: Killer cell immunoglobulin like receptor gene association with tuberculosis. Hum Immunol 2013, 74(1):85-92.

44. Majorczyk E, Pawlik A, Łuszczek W, Nowak I, Wiśniewski A, Jasek M, Kuśnierczyk P: Associations of killer cell immunoglobulin-like receptor genes with complications of rheumatoid arthritis. Genes \& Immunity 2007, 8(8):678-683.

45. Kulkarni S, Martin MP, Carrington M: The Yin and Yang of HLA and KIR in human disease. Semin Immunol 2008, 20(6):343-352.

46. Katz G, Gazit R, Arnon TI, Gonen-Gross T, Tarcic G, Markel G, Gruda R, Achdout H, Drize O, Merims S et al: MHC Class I-Independent Recognition of NK-Activating Receptor KIR2DS4. The Journal of Immunology 2004, 173(3):1819.

47. Naumova E, Mihaylova A, Stoitchkov K, Ivanova M, Quin L, Toneva M: Genetic polymorphism of NK receptors and their ligands in melanoma patients: prevalence of inhibitory over activating signals. Cancer Immunol Immunother 2005, 54(2):172-178

48. Clements JE, Gama L, Graham DR, Mankowski JL, Zink MC: A simian immunodeficiency virus macaque model of highly active antiretroviral treatment: viral latency in the periphery and the central nervous system. Curr Opin HIV AIDS 2011, 6(1):37-42.

49. Dinoso JB, Rabi SA, Blankson JN, Gama L, Mankowski JL, Siliciano RF, Zink MC, Clements JE: A Simian Immunodeficiency Virus-Infected Macaque Model To Study Viral Reservoirs That Persist during Highly Active Antiretroviral Therapy. Journal of Virology 2009, 83(18):9247.

50. Marquardt N, Beziat V, Nystrom S, Hengst J, Ivarsson MA, Kekalainen E, Johansson H, Mjosberg J, Westgren M Lankisch TO et al: Cutting edge: identification and characterization of human intrahepatic CD49a+ NK cells. J Immunol 2015, 194(6):2467-2471.

51. Marquardt N, Scharenberg M, Mold JE, Hård J, Kekäläinen E, Buggert M, Nguyen S, Wilson JN, Al-Ameri M Ljunggren $\mathrm{H}-\mathrm{G}$ et al: Expansions of adaptive-like NK cells with a tissue-resident phenotype in human lung and blood. bioRxiv 2020:2019.2012.2020.883785.

52. Sojka DK, Yang L, Yokoyama WM: Uterine Natural Killer Cells. Front Immunol 2019, 10:960.

53. Poggi A, Benelli R, Venè R, Costa D, Ferrari N, Tosetti F, Zocchi MR: Human Gut-Associated Natural Killer Cells in Health and Disease. Front Immunol 2019, 10:961.

54. Sojka DK, Plougastel-Douglas B, Yang L, Pak-Wittel MA, Artyomov MN, Ivanova Y, Zhong C, Chase JM, Rothman $\mathrm{PB}, \mathrm{Yu} \mathrm{J}$ et al: Tissue-resident natural killer (NK) cells are cell lineages distinct from thymic and conventional splenic NK cells. Elife 2014, 3:e01659.

55. Dogra P, Rancan C, Ma W, Toth M, Senda T, Carpenter DJ, Kubota M, Matsumoto R, Thapa P, Szabo PA et al: Tissue Determinants of Human NK Cell Development, Function, and Residence. Cell 2020, 180(4):749-763. e713.

56. Ferreira PG, Muñoz-Aguirre M, Reverter F, Sá Godinho CP, Sousa A, Amadoz A, Sodaei R, Hidalgo MR, Pervouchine D, Carbonell-Caballero J et al: The effects of death and post-mortem cold ischemia on human tissue transcriptomes. Nature Communications 2018, 9(1):490.

57. Li Y, Wang T, Hu X, Zhang H, Chen L, Bao X, He J: Study of KIR gene expression at the mRNA level in specific donor-derived NK cells after allogeneic HSCT. Immunogenetics 2020, 72(3):135-141.

58. Küçük C, Hu X, Gong Q, Jiang B, Cornish A, Gaulard P, McKeithan T, Chan WC: Diagnostic and Biological Significance of KIR Expression Profile Determined by RNA-Seq in Natural Killer/T-Cell Lymphoma. Am J Pathol 2016, 186(6):1435-1441. 




\section{Appendices}

Summary

Nederlandse samenvatting

Dankwoord

Curriculum vitae

List of Publications 


\section{Summary}

Natural killer (NK) cells represent a major component of the immune system, and are involved in the protection against pathogens, the surveillance of tumor cells, and the regulation of other players in the immune response. The education, activation and functioning of NK cells is tightly modulated by two major sets of inhibitory and activating receptors. A conserved set of NK cell receptors include members of the CD94:NKG2 family that recognize MHC-E molecules. The other set comprise the killer cell immunoglobulin-like receptors (KIR), which represent a diverse group of structurally similar transmembrane molecules. Members of the KIR family co-evolved with their diversified MHC-A,-B and-C ligands that are in a continuous arms race with pathogens. The KIR receptors are encoded within the Leukocyte Receptor Complex (LRC) on chromosome 19q13.4, and segregate independent from their $\mathrm{MHC}$ ligands. The KIR genes are arranged in a polygenic cluster conform a head-to-tail tandem organization, which is characterized by extensive diversification generated by point mutations and chromosomal recombination. As such, the KIR gene content is highly unique at an individual level and displays differential gene distribution at a population level. The highly variable KIR gene content, in combination with their polymorphic MHC class I ligands, is associated with differential susceptibility and protection in health and disease.

In this thesis, we aimed to improve the KIR gene characterization in humans and macaques. The latter species is commonly used as model in preclinical studies to develop medicine and therapies. Their KIR gene system and NK cell biology display similarities to humans, with species-specific variation mainly reflected in the KIR receptor structure and haplotype organization. Initial transcriptome characterization studies using conventional sequencing techniques indicated a highly plastic KIR gene system in macaques that might exceed the complexity observed in humans. However, a comprehensive overview of the macaque KIR gene system is lacking.

Conventional sequencing techniques, such as Roche 454 and Sanger sequencing, are limited to relatively short fragments. These techniques might be used to determine the presence or absence of KIR genes, or to define partial and common full-length transcripts at an allele level resolution. Nevertheless, the conventional approaches have an insufficient sequencing depth to obtain a comprehensive characterization of complex systems, such as KIR. A more thorough characterization of the KIR gene system is achieved by the application of single-molecule real-time (SMRT) sequencing platforms, which are commercialized by Pacific Biosciences (PacBio) and Oxford Nanopore Technologies (ONT). The high accuracy and throughput of PacBio circular consensus sequencing allowed the complete definition of full-length KIR transcriptomes in humans and rhesus macaques at the allele level resolution (Chapter 2). A familybased study design and subsequent segregation analysis enabled the deduction of KIR haplotype configurations. In humans, these configurations were conforming the common centromeric and telomeric regions, with either more inhibitory or activating gene content ( $\mathrm{A}$ or $\mathrm{B}$ ). A more dynamic gene content variability is featured in rhesus 
macaques, as reflected by expansions, contractions, and the generation of substantial numbers of fusion genes. The differences between the human and rhesus macaque $K I R$ gene systems are in line with the evolutionary distance to a common ancestor, approximately 25 million years ago. The rapid pace of $K I R$ gene evolution is also illustrated by the comparison of the closely related rhesus and cynomolgus macaques, and their distinct populations (Chapter $\mathbf{3}$ ). The presence of abundant species- and population-specific macaque KIR genes indicate an unparalleled rapid evolution, which is probably propelled by the rapid adaption to differential pathogenic selective pressures. The diversification is largely mediated through chromosomal recombination events, which may shuffle head and tail segments of KIR genes, but also complete KIR haplotype segments.

The KIR transcriptome studies not only resolved the KIR gene diversity and allelic polymorphism, but also unravelled a wide spectrum of alternative splicing profiles (Chapter 4). Even though some of the alternative splicing events might represent transcriptional noise, the conservation of different splice events and the relatively large number of spliced transcripts in the transcriptome studies, suggest that at least several isoforms may have functional relevance. The variety of structurally diverged receptors might, for example, alter their ligand interactions, cellular localization, expression level, and signalling properties. The wide range of KIR isoforms extends the plasticity of the KIR gene cluster in primates.

The KIR transcriptome studies revealed information on allelic variants, copy number variation, chromosomal recombination events, haplotype configurations, and alternative splicing. However, these studies lack insights on the genomic organization of this complex gene cluster. We developed a Cas9-mediated target enrichment protocol to characterize complete KIR haplotypes in humans and rhesus macaques (Chapter 5). Using this strategy, the KIR haplotype architecture, the non-coding stretches, such as promotor regions and introns, and the epigenetic profiles were resolved.

The dynamics of the primate $K I R$ gene system relies on multiple molecular mechanisms that together generates a compound array of diversity at an individual, population, and species level (Chapter 6). This molecular toolbox that facilitates rapid evolution is shared in the different primate species, which demonstrates an evolutionary effort to diversify their KIR gene cluster. The improved insights into the genomic and transcriptomic KIR profiles, and the development of novel high-throughput techniques, pave the way to examine functional implications of the dynamic KIR cluster in macaque models for health and disease (Chapter 7). This knowledge might translate to a better understanding of the human NK cell biology and related disease phenotypes. 


\section{Nederlandse samenvatting}

Natural killer (NK) cellen vormen een belangrijk onderdeel van het immuunsysteem, en zijn betrokken bij de bestrijding van pathogenen, de detectie van tumorcellen en de regulatie van andere immuun componenten. De educatie, de activatie en het functioneren van NK-cellen worden strikt gemodelleerd door twee verschillende receptor groepen, welke beide activerende en inhiberende signalen kunnen doorgeven. De geconserveerde groep bestaat uit leden van de CD94:NKG2 receptorfamilie, welke een interactie kunnen aangaan met MHC-E moleculen. De andere groep omvat de killer cell immunoglobulin-like receptoren (KIR), welke grote variatie vertonen, maar structureel verwant zijn aan elkaar. De KIR receptoren en hun zeer diverse MHC-A,-B, en $-C$ liganden, die in een constante adaptieve strijd zijn met pathogenen, zijn onderhevig aan co-evolutie. De genen die voor de KIR receptoren coderen liggen op chromosoom 19q13.4 en segregeren onafhankelijk van hun MHC liganden. De KIR genen liggen in een kop-staart organisatie gerangschikt binnen een polygenetisch cluster. Deze gen regio ondergaat veelvuldige en uiteenlopende diversificatie, aangedreven door puntmutaties en chromosomale recombinaties. Hierdoor is de combinatie van aanwezige KIR genen tot op zekere hoogte uniek per individu, en zijn genen en allelische variaties verschillend verdeeld in geografische populaties. De verscheidenheid van KIR genen, in combinatie met hun polymorfe MHC klasse I liganden, wordt geassocieerd met de vatbaarheid voor en de bescherming tegen bepaalde ziektes. Daarnaast zijn verschillende KIR en MHC combinaties in verband te brengen met de regulatie van biologische processen, zoals zwangerschap.

In deze thesis streven wij naar een verbetering van de karakterisatie van de KIR genen in de mens en makaaksoorten. De laatstgenoemde diersoorten worden op een regelmatige basis gebruikt als model in preklinische studies om medicijnen en therapieën te ontwikkelen. Het makaak KIR gen systeem en hun NK cel biologie vertoont veel gelijkenissen met dat van de mens. Diersoort-specifieke kenmerken kunnen vooral gevonden worden in de receptor structuur en de haplotype organisatie. De eerste studies voor het in kaart brengen van KIR transcripten gebeurde met behulp van conventionele sequencing methodes en indiceerde een complex systeem met meer genetische diversiteit in de makaak dan in de mens. Echter, een omvangrijk overzicht van het KIR gen cluster in de makaak is niet beschikbaar.

Conventionele sequencing technieken, zoals Roche 454 en Sanger sequencing, zijn beperkt tot het sequensen van korte fragmenten. Daarmee zijn deze methodes geschikt om de aan- en afwezigheid van genen te bepalen of om partiele en veelvoorkomende gehele transcripten te identificeren op allel niveau. Desalniettemin zijn deze technieken niet toereikend genoeg om een uitgebreid overzicht van het gehele KIR gen systeem te geven. Een meer gedetailleerd beeld kan verkregen worden met behulp van single-molecule real-time (SMRT) sequencing platforms, zoals Pacific Biosciences (PacBio) en Oxford Nanopore Technologies (ONT). De relatief hoge accuraatheid en output van PacBio circular consensus sequencing (CCS) maakte het mogelijk om humane en makaak KIR genen volledig in kaart te brengen op allel niveau 
resolutie (Hoofdstuk 2). Door een familie-gebaseerd onderzoeksopzet en segregatie analyses konden KIR haplotype configuraties worden afgeleid. In de mens volgen de configuraties een standaard organisatie, zowel op de centromere als telomere segmenten, met of meer inhiberende of meer activerende genen ( $\mathrm{A}$ of $\mathrm{B}$ ). Daarentegen kenmerken de KIR configuraties in de resus makaak een meer dynamische content, waarbij expansie, contractie, en de formatie van fusie genen voor variatie zorgen. Met een evolutionaire afstand tot een gemeenschappelijke voorouder van ongeveer 25 miljoen jaar zijn de verschillen tussen het KIR gen systeem in de mens en makaak in de lijn der verwachtingen. De snelheid waarmee het KIR gen systeem evolueert is ook duidelijk zichtbaar bij de vergelijking tussen twee nauwverwante makaaksoorten, de resus en cynomolgus makaak, en hun verschillende populaties (Hoofdstuk 3). De grote diversiteit aan diersoort- en populatie-specifieke makaak KIR genen tonen een ongekend snelle evolutie aan, welke waarschijnlijk wordt aangedreven door evolutionaire selectie onder een uiteenlopende pathogene druk. Deze diversificatie wordt grotendeels gemedieerd door chromosomale recombinatie, waarbij de kop en de staart van genen kunnen worden uitgewisseld, maar ook complete haplotype segmenten kunnen worden gereorganiseerd.

Het karakteriseren van de KIR transcriptie gaf echter niet alleen inzicht in de KIR gen diversiteit en de allelische variatie, maar ontrafelde ook een wijd spectrum aan alternatieve splicing profielen (Hoofdstuk 4). Een deel van de alternatieve splicing gebeurtenissen zijn mogelijk ruis van het transcriptie proces. Echter, de conservatie van bepaalde splicing gebeurtenissen, en de aanwezigheid van een relatief grote hoeveelheid aan alternatieve sequenties in de transcriptie studies, suggereren dat een deel van de isovormen een functionele relevantie hebben. De verscheidenheid aan structureel verschillende receptoren kunnen bijvoorbeeld van invloed zijn op ligand interacties, de lokalisatie in de cel, de expressie niveaus, en de signaaltransductie. Hiermee wordt de plasticiteit van de KIR genen in primaten verder verruimd.

De KIR transcriptie studies geven voornamelijk een beeld van het polymorfisme, copy nummer variatie, chromosomale recombinatie, haplotype configuraties, en alternatieve splicing, maar de genomische structuur van het cluster blijft hiermee vooralsnog onduidelijk. Daarom hebben wij een Cas9-gemedieerde methode ontwikkelt om specifiek de KIR regio te kunnen verrijken en daarmee de genomische organisatie van KIR haplotypes in de mens en de makaak te kunnen bepalen (Hoofdstuk 5). Deze strategie maakte het mogelijk om de volgorde van KIR genen op een haplotype, de niet-coderende segmenten, zoals de intronen en promotor regio's, en de epigenetische profielen in kaart te brengen.

De dynamiek van de KIR regio in primaten berust op verschillende moleculaire mechanismes, welke tezamen een uitgebreid pallet aan diversiteit genereerd op individu, populatie en diersoort niveau (Hoofdstuk 6). Deze set aan mechanismes faciliteert een snelle evolutie en is geconserveerd in primaten. Dit wijst erop dat er een evolutionaire selectiedruk is om de $K I R$ regio divers te houden. Met de in dit proefschrift verworven inzichten op het $K I R$ gen profiel in primaten en met de verdere doorontwikkeling van nieuwe sequencing technieken, ontstaan er mogelijkheden om 
Nederlandse samenvatting

de functionele implicaties van de dynamische KIR receptor familie gedetailleerder te onderzoeken (Hoofdstuk 7). Dit brengt een mogelijke vertaalslag van de kennis die wordt verkregen uit makaak studies naar een beter begrip van de menselijke NK cel biologie en gerelateerde ziektefenotypes. 


\section{Dankwoord}

Meer dan vijf jaar aan werk is opgeschreven in dit proefschrift, wat tot stand is gekomen door het isoleren van DNA uit miljoenen humane en makaak cellen, het op volgorde zetten van zeker een miljard nucleotiden, en het gebruik maken van ontelbare uren aan rekenkracht. Al dit werk zou absoluut onmogelijk zijn geweest zonder de directe en indirecte steun van mensen uit mijn omgeving. Met dit dankwoord wil ik iedereen bedanken die mij in de afgelopen jaren heeft bijgestaan met raad en daad. Maar uiteraard is dit ook de plaats waar ik een aantal personen in het bijzonder kan uitlichten.

Allereerst wil ik jou bedanken, Ronald. Nog altijd vertel jij vol bewondering mooie verhalen over jouw toenmalige promotor, Prof. Dr. Jon van Rood. Als mijn promotor ben jij in zijn voetsporen getreden en kan ik op eenzelfde manier teren op jouw levendige anekdotes, wijze lessen en academische visie. Het vertrouwen dat je in mij stelde sinds de eerste dag waardeer ik enorm. Elke keer wanneer ik uit wetenschappelijke nieuwsgierigheid een nieuwe techniek of methode voorstelde, gaf je mij de ruimte om het in praktijk te brengen. Ondanks dat er in theorie geen verschil zit tussen theorie en praktijk, was dit in de praktijk vaak wel het geval. Dit hield het onderzoek in ieder geval uitdagend. Ondanks je drukke schema benadrukte je altijd de "open deur" cultuur, waarin ik kon binnen lopen om resultaten te bespreken of voor een simpel praatje over voetbal. Het werk op jouw afdeling kreeg nog extra glans door de diverse congressen, waar je mij introduceerde aan wetenschappers in het veld en waar ik ons werk mocht presenteren voor een groot publiek. In de afgelopen jaren heb ik onder jouw begeleiding mijn grenzen kunnen verleggen en ben ik ontwikkeld op persoonlijk en wetenschappelijk vlak. Met het vertrouwen dat je in mij uitspreekt door de aanstelling als vaste medewerker in de Bontrop-groep hoop ik op nog vele wetenschappelijke discussies, welke leiden tot mooie publicaties voor onze afdeling. Wie schrijt, die blijft...

Minstens zo belangrijk tijdens mijn promotie was jij, Natasja. Als de eerste PhD student die jij hebt begeleid kan ik je vertellen dat je trots mag zijn. In de eerste maanden heb jij met geduld mij wegwijs gemaakt in de moleculaire technieken en ook in de daaropvolgende jaren heb je mij altijd voorzien van praktisch advies. Elke keer als we weer een stapje dichterbij een mooi resultaat kwamen, kon ik bij jou binnen lopen om de data te bespreken en de vervolgstappen te bediscussiëren. Ik waardeer de kritische en gedetailleerde aanpak waarmee jij mijn manuscripten van commentaar hebt voorzien, wat vervolgens heeft geleid tot mooie publicaties. Sinds het vertrek van Gaby heb je laten zien dat je een goede manager bent op de afdeling, waar ik een voorbeeld kan nemen aan jouw gestructureerde en rustige manier van leiding geven. Gedurende het PhD traject zijn we steeds meer naar eenzelfde niveau gegroeid en ik kijk ernaar uit om samen met jou de afdeling verder door te ontwikkelen. 
De grote hoeveelheid data dat is verwerkt in dit proefschrift heb ik natuurlijk nooit alleen kunnen genereren en verwerken. Daarvoor is een geolied en saamhorig team nodig dat voor elkaar klaar staat. Ik ben enorm dankbaar dat ik me bij dit team heb mogen voegen en dat ik heb mogen ervaren hoe jullie met plezier aan de slag gaan. Jullie bijdrage was niet louter wetenschappelijk... Met alle verhalen over jullie kinderen ben ik de meest ervaren kinderloze opvoedkundige geworden!

Marit, in de afgelopen jaren heb je veel bijgedragen aan het KIR onderzoek; eerst bij Jeroen en daarna met mij. Je beheerst de complexiteit van het gen systeem tot in detail en hierdoor kon je altijd kritisch meedenken over de praktische uitvoering van onze projecten. Ik bewonder hoe snel je nieuwe technieken oppakt en zelf aan de slag gaat met ingewikkelde data-analyses. Samen hebben we veel opgehelderd over de ingewikkelde wirwar aan genen en je mag trots zijn op jouw grote bijdrage aan dit proefschrift!

Het verbaast me dat jij niet duizelig wordt achter de computer, Nanine. De grote tabellen om de KIR haplotypes te definiëren zijn zeer waardevol en daarmee heb ook jij een grote bijdrage geleverd aan verschillende hoofdstukken van dit proefschrift. Er is geen analist die zo snel en zo nauwkeurig de PacBio data kan verwerken. Heel veel dank voor dit werk!

Op onze afdeling is het eigenlijk vanzelfsprekend dat de voorraad aan cellen en geïsoleerd DNA/RNA op orde is. Maar dit ligt er natuurlijk niet zomaar. Daarom wil ik ook jou bedanken, Annemiek, voor alle opwerkingen die jij in de afgelopen jaren hebt verricht.

Hoe zeer ik stilte en rust waardeer tijdens het schrijven van artikelen, was het fijn om toch een vast kamergenootje te krijgen. Lisanne, ik waardeer de gesprekken op de kamer en de discussies tijdens de PhD overleggen. Ook had ik zonder jou waarschijnlijk na vijf jaar nog steeds in een kale lege kamer gezeten, dus ik ben blij met de plantjes die ons zijn komen vergezellen! Nu nog de taak om ze in leven houden...

Corinne, ondanks ons werk misschien pas recentelijk wat meer overlap heeft gekregen nu je ook onderzoek doet naar het KIR systeem in de chimpansees, wil ik ook jou bedanken voor je input tijdens mijn onderzoek.

En dan nog de twee ex-CGR'ers. Allereerst Nel, je nuchtere blik op vraagstukken kon ik altijd erg waarderen en soms ook wel om lachen. Ik ben blij dat je me hebt geholpen met de vele allel submissies naar de database en ik hoop dat je inmiddels geniet van je pensioen! En ook jou, Gaby, wil ik bedanken voor de adviezen in de eerste jaren van mijn PhD. Als initiator van het PacBio sequencen op het BPRC heb je toch een hoeksteen gelegd van dit manuscript.

Al met al dus een heel waardevol team op de genetica afdeling, en zonder jullie was dit onderzoek nooit zo ver gekomen, en was het werken ook nooit zo fijn geweest. Bedankt! En hopelijk volgen er nog vele mooie onderzoeken samen als team.

Ondanks dat ik mijn promotietraject afsluit in een bijzondere tijd, waarbij contact met medewerkers van andere afdelingen minimaal was, wil ik ook deze collega's bedanken. Als eerst de groep post-docs die de tijd nam om actief mee te doen met de 
Journal Club. Ondanks dat mijn cel-immunologische kennis soms niet even toereikend was, heb ik veel geleerd van deze bijeenkomsten. Dus dank daarvoor Ed, Frank, Bart, Gerrit, Michel, Marieke, Kirsta en Jeffrey. En daarmee wil ik ook meteen mijn dank richten tot mijn mede-lotgenoten: Karin, Jordon, Raissa, Lisanne, Annemarie, Kinga, Aafke, Astrid, en Tina, voor de gezellige etentjes in Delft en het delen van hun ervaringen. Het KIR onderzoek heeft veel gevraagd van onze computer systemen. Ik wil Doxx, en dan in het bijzonder Billy, enorm bedanken voor hun ondersteuning in het opzetten van de Linux/Windows computer waarop het Nanopore platform draait en alle support die ze daarbij hebben verleend. Verder nog mijn dank voor een aantal mensen die misschien wel het meeste inzicht hebben van het hele instituut. Want ondanks dat het BPRC gelegen is in een regio waar het slecht is gesteld met de kennis over voetbal, zijn er toch collega's die beter weten. Daarom ook mijn dank aan Dirk, Jeffrey en Paul, die altijd de tijd namen om de wedstrijden van onze landskampioen te analyseren. Uiteraard ook nog een dank aan Francisca. Je hebt met veel geduld al mijn figuren ontworpen, waarbij ik vaak terugkwam om nog de kleinste details te laten aanpassen. Heel erg bedankt voor de tijd die jij hebt gestoken in het ontwerpen van de figuren en het vormgeven van dit proefschrift.

The many congresses in the most beautiful places introduced me to inspiring scientists in the field of immunogenetics and NK cells. It is amazing to be part of this community and I am thankful for the warm welcome. Specifically, I would like to thank prof. Dr. Peter Parham, prof. Dr. Lutz Walter and Prof. Dr. Jim Kaufman for taking the time to read my thesis and to take part in the defense-committee. Uiteraard wil ik ook de andere twee professoren die mijn proefschrift willen beoordelen bedanken, prof. Dr. Edwin Cuppen en prof. Dr. Rob de Boer. It is an honor to defend my thesis in front of these well-established scientists.

Naast de zeer gewaardeerde collega's zou ik ook graag mijn dank willen richten aan vrienden en familie. Ondanks dat vele hun directe en indirecte bijdrage hebben gehad in het behalen van mijn doctoraat, wil ik een select aantal in het bijzonder noemen.

Remi, jij bent iemand die altijd voor iedereen klaar staat en je bent van grote waarde geweest in mijn periode als PhD'er. Ondanks dat je zelf moeilijke tijden hebt doorgemaakt liet je je nooit uit het veld slaan, en kon ik altijd bouwen op je realistische en positieve kijk op verschillende onderwerpen. De avonden met lekker eten, bordspelletjes, drank en je zwarte humor zijn geweldig. En hier hoort Sanne natuurlijk ook bij. Jouw kookkunsten, enthousiaste verhalen en zorgzame karakter maken je een bijzonder persoon. Dank dat ik altijd op jullie kan rekenen! En als vriend die ik al bijna 25 jaar ken, Dwayne, ben jij ook een belangrijk persoon geweest in de afgelopen jaren. Met je uitsloverij tijdens de wintersport en je soms wat rare streken ben je nog altijd het jochie van de Flierefluiter. Bedankt voor de gezellige momenten bij jou en Esther thuis, en mooi om te zien hoe de kwajongen nu doorgaat als trotse vader. Ruben, een vriendschap die een gekke oorsprong kent, maar zich wel ontwikkelt tot iets waardevols. Als nieuwe mug in Haarlem is het fijn om soms een biertje te kunnen doen en simpelweg te kunnen zeuren op onzinnige dingen. 
Soms gaan dingen in het leven ook heel anders dan verwacht... Toch wil ik jou, Esther, bedanken voor de grote steun die je bent geweest tijdens het doen van mijn onderzoek. Inmiddels weet je zelf hoe het is om een promotie traject te doorlopen en zal je begrijpen dat het soms lekker is om even op het onderzoek te kunnen zeuren. Het is fijn dat je in die periode een luisterend oor kon zijn en dat je me altijd motiveerde om door te gaan. De reisjes naar zonnige stranden en etentjes bij de voor ons welbekende restaurants waren mooie momenten om het werk te vergeten. Met jouw ambitie weet ik zeker dat je ook je eigen dromen zal najagen en uiteindelijk als zeergeleerde vrouw door het leven zal gaan.

Als laatste wil ik mijn familie bedanken. Mads en Demi, jullie beeld van mij, werkende in een witte jas op een laboratorium in een apencentrum, is natuurlijk al heel accuraat. Hopelijk kunnen jullie met het lezen van dit manuscript dat beeld nog wat verfijnen! Lieve papa en mama, jullie hebben altijd duidelijk laten blijken trots te zijn op mijn werk en vaak gevraagd naar nieuwe publicaties. Dit heeft mij erg gemotiveerd. Zoals jullie graag willen, en in tegenstelling tot mijn universitaire diploma's, zal ik deze mijlpaal wel vieren! 


\section{Curriculum Vitae}

Jesse Bruijnesteijn was born on the 28th of February 1991 in Hoorn, the Netherlands. In 2009 he obtained his VWO diploma (Copernicus, Atlas College, Hoorn). In the same year he started his bachelor's in Biomedical Sciences at the University of Amsterdam (UvA). He complemented these years with extracurricular courses in Chemistry and World politics. Following his Bachelor's degree, he started the master Drugs Discovery and Safety: Analytical Chemistry at the Vrije Universiteit (VU) in Amsterdam. This programme involved an internship at the VU medical center (Amsterdam) during which he developed a method to define and quantify corticosteroids in the faeces of preterm neonates using a liquid chromatography and mass spectrometry (LC-MS) protocol. Next, he wrote a literature thesis in collaboration with MSD-Merck on the two commercially available PD-1 blockers (Pembrolizumab and Nivolumab) to treat advanced melanomas and non-small-cell lung cancer.

After his graduation, he started at the Biomedical Primate Research Centre (BPRC; Rijswijk, the Netherlands) as PhD candidate at the department of Comparative Genetics and Refinement. This study involved the comprehensive characterization of killer cell immunoglobulin-like receptors (KIR) in macaque species and humans. 


\section{List of publications}

Jesse Bruijnesteijn, Marit K. H. van der Wiel, Wendy T. N. Swelsen, Nel Otting, Annemiek J. M. de Vos-Rouweler, Diënne Elferink, Gaby G. Doxiadis, Frans H. J. Claas, Neubury M. Lardy, Natasja G. de Groot and Ronald E. Bontrop. (2018) Human and Rhesus Macaque KIR Haplotypes Defined by Their Transcriptomes. J Immunol, 200 (5) 1692-1701.

Jesse Bruijnesteijn, Marit K. H. van der Wiel, Nanine de Groot, Nel Otting, Annemiek J. M. de Vos-Rouweler, Neubury M. Lardy, Natasja G. de Groot and Ronald E. Bontrop. (2018) Extensive Alternative Splicing of KIR Transcripts. Front Immunol 9, 2846.

Nanine de Groot; Rens Groen; Vaneesha Orie; Jesse Bruijnesteijn; Natasja G. de Groot; Gaby G.M. Doxiadis; Ronald E. Bontrop (2019). Analysis of macaque BTN3A genes and transcripts in the extended $\mathrm{MHC}$ : conserved orthologs of human $\gamma \delta \mathrm{T}$ cell modulators. Immunogenetics, volume 71, issue 8-9, pp. $545-559$.

Jesse Bruijnesteijn, Natasja G. de Groot, Nel Otting, Giuseppe Maccari, Lisbeth A. Guethlein, James Robinson, Steven G. E. Marsh, Lutz Walter, David H. O'Connor, John A. Hammond, Peter Parham \& Ronald E. Bontrop. (2019) Nomenclature report for killer-cell immunoglobulin-like receptors (KIR) in macaque species: new genes/alleles, renaming recombinant entities and IPD-NHKIR updates. Immunogenetics volume 72 , issue 1-2, pp. 37-47.

Jesse Bruijnesteijn, Nanine de Groot, Marit K. H. van der Wiel, Nel Otting, Annemiek J. M. de Vos-Rouweler, Natasja G. de Groot and Ronald E. Bontrop. (2020) Unparalleled Rapid Evolution of KIR Genes in Rhesus and Cynomolgus Macaque Populations. J Immunol, 204 (7) 1770-1786.

Jesse Bruijnesteijn, Natasja G. de Groot and Ronald E. Bontrop. (2020) The Genetic Mechanisms Driving Diversification of the KIR Gene Cluster in Primates. Front. Immunol 11, 582804.

Natasja G. de Groot, Corrine M. C. Heijmans, Suzanne Bezstarosti, Jesse Bruijnesteijn, Geert W. Haasnoot, Arend Mulder, Frans H. J. Claas, Sebastiaan Heidt and Ronald E. Bontrop. (2021) Two Human Monoclonal HLA-Reactive Antibodies Cross-React with Mamu-B*008, a Rhesus Macaque MHC Allotype Associated with Control of Simian Immunodeficiency Virus Replication. J Immunol 206 (8), 1957-1965.

Jesse Bruijnesteijn, Marit van der Wiel, Natasja G. de Groot, and Ronald E. Bontrop. (2021) Rapid characterization of complex killer cell immunoglobulin-like (KIR) regions using Cas9 enrichment and Nanopore sequencing. Accepted for publication in Front. Immunol. 

